\title{
TRANSIENT BEHAVIOR OF THE PLANAR-FLOW MELT SPINNING PROCESS WITH CAPILLARY DYNAMICS
}

\author{
A Dissertation \\ Presented to the Faculty of the Graduate School \\ of Cornell University \\ in Partial Fulfillment of the Requirements for the Degree of \\ Doctor of Philosophy
}

Eric Alan Theisen

May 2007 
(C) 2007 Eric Alan Theisen

ALL RIGHTS RESERVED 


\title{
TRANSIENT BEHAVIOR OF THE PLANAR-FLOW MELT SPINNING PROCESS WITH CAPILLARY DYNAMICS
}

\author{
Eric Alan Theisen, Ph.D. \\ Cornell University 2007
}

Planar-flow melt spinning (PFMS) is a single-stage rapid manufacturing technique for producing thin metal sheets or ribbons. During the processing molten metal flows through a nozzle onto a moving substrate where a puddle is formed. This study focuses on the time dependent behavior of the process and looks at the puddle dynamics during the cast. Understanding how the issues of heat transfer, fluid flow and contacting dynamics influence the quality of the cast ribbon are the primary focus of the study. The commercial acceptance of PFMS requires ribbons to be cast with good quality (e.g. uniform thickness).

There are a large range scales that are relevant to the ribbon product. Thickness variations occur on the macro-scale over the length of the cast $(50 \mathrm{~m})$. There is a steady decrease in ribbon thickness over the length of the cast. There is also a periodic variation on the length scale of the wheel circumference $(2-3 \mathrm{~m})$. Steady mass, momentum and energy balances are used to understand these long lengthscale thickness variations.

There are also thickness variations that occur on smaller scales, $\mathrm{cm}$ to $\mathrm{mm}$. A periodic thickness variation across the width of the ribbon is a common defect observed in our casting, referred to as the cross-wave defect. The molten metal 
puddle is subject to capillary vibrations. We find that these oscillations correspond to the cross-wave defect.

Motivated by the physics of the cross-wave defect, a more generalized problem of the vibrations of coupled capillary surface is identified. The motions of the puddle are similar to the classical problem of the vibration modes of a 'plucked' sphere. In considering the possible modes of vibration for a droplet confined between two plates, we find a novel vibration mode where the center-of-mass oscillates. This model is extended to account for a droplet confined within a tube, with two sections of the droplet extending from each end. Restricting to quasi-steady interface shapes, we find a translational vibration mode which can occur with lower frequency than the traditional Rayleigh modes. The vibration frequencies are compared to experimental values. 


\section{BIOGRAPHICAL SKETCH}

The author was born in Winkler, Manitoba Canada and raised in North Dakota.

He attended the University of Florida, earning a BS in chemical engineering in May 2001. He entered Cornell University the following August. 


\section{ACKNOWLEDGEMENTS}

I would like to thank my advisor, Paul H. Steen, whose guidance was invaluable. Paul has been a mentor to me, but more importantly, a friend. He has helped me through some difficult times in my life, and I will always be grateful to him. Thanks are also due to the other members of my committee, Timothy J. Healey (T\&AM), Nicholas J. Zabaras (MechE), and Steven J. Weinstein (Eastman Kodak

and RPI) for taking the time to serve. Steve has been a great motivator of ideas and has helped to put my research in perspective.

I would also like to thank Cormac Byrne and Mike Vogel for their help with my research, as well as their friendship.

My research has been partially supported by an Eastman Kodak Graduate Research Fellowship. Support by NASA NAG3-2713, -2714, NSF DMI-0500311, -0500408, ARO and DARPA is gratefully acknowledged. 


\section{TABLE OF CONTENTS}

1 Planar-flow Melt Spinning and Capillary Vibrations Background 1

1.1 Introduction . . . . . . . . . . . . . . . . . . . 1

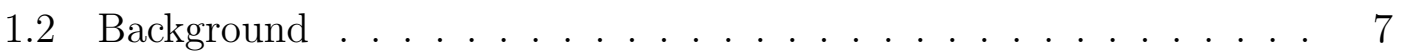

1.3 Literature review . . . . . . . . . . . . . . . . . . 11

1.3.1 Steady state behavior . . . . . . . . . . . . . . 12

1.3.2 Time dependent behavior . . . . . . . . . . . . 16

1.3 .3 Droplet vibrations . . . . . . . . . . . . . . . . . . . . 22

1.4 Summary of this study . . . . . . . . . . . . . . 24

2 Transient Behavior of the Planar-flow Melt Spinning Process 38

2.1 Introduction . . . . . . . . . . . . . . . 38

2.2 Experimental results . . . . . . . . . . . . . . . . . . . 40

2.2.1 Caster operation and data acquisition . . . . . . . . . . 40

2.2 .2 Experimental data . . . . . . . . . . . . . . . 42

2.3 Modeling of the process dynamics . . . . . . . . . . . . 45

2.3.1 Wheel heat-up and expansion . . . . . . . . . . . 48

2.3.2 Dynamical model of puddle size . . . . . . . . . . . . . . 52

2.3.3 Steady Bernoulli balance . . . . . . . . . . . . . . . . 57

2.3.4 Solidification velocity . . . . . . . . . . . . . . . . 57

2.4 Results . . . . . . . . . . . . . . . . . . . . . . . . . 59

2.5 Discussion . . . . . . . . . . . . . . . . . . 63

2.6 Conclusion . . . . . . . . . . . . . . . . . . . . 68

2.7 Data appendix . . . . . . . . . . . . . . . . . . 69

3 Capillary Puddle Vibrations Linked to Casting-defect Formation in Planar-flow Melt Spinning $\quad 77$

3.1 Introduction . . . . . . . . . . . . . . 77

3.2 Template- vs. pulse-transfer . . . . . . . . . . . . . . . . . . . . . 82

3.3 Apparatus and experimental methods . . . . . . . . . . . . 83

3.4 Puddle oscillations . . . . . . . . . . . . . . . . . . . 87

3.5 Puddle shape deviations* $\ldots \ldots \ldots \ldots$. . . . . . . . . 92

3.6 Cross-wave physics . . . . . . . . . . . . . . . . . . 94

3.7 Capillary vibrations of a fluid confined in a narrow channel ${ }^{*}$. . . . 99

3.7.1 Model problem . . . . . . . . . . . . . . . . . . 100

3.7.2 Effect of the static contact angle . . . . . . . . . . . 104

3.7.3 Effect of the wetting condition . . . . . . . . . . . 105

3.8 Defect formation mechanism* . . . . . . . . . . . . . 106

3.9 Thresholds for cross-wave appearance . . . . . . . . . . . . . 108

3.10 Discussion . . . . . . . . . . . . . . . . . . . . . . . 113

3.11 Conclusion . . . . . . . . . . . . . . . . . . . 116

3.12 Acknowledgements . . . . . . . . . . . . . . . . . 118 
3.13 Appendix . . . . . . . . . . . . . . . . . . . 118

3.13.1 Time dependent behavior of gap height . . . . . . . 118

4 Capillary Dynamics of Coupled Spherical-cap Droplets 123

4.1 Overview . . . . . . . . . . . . . . . . . . . . . . . . . . . . . 123

4.2 Dynamical model . . . . . . . . . . . . . . . . . . . 126

4.2.1 Phase-plane solution . . . . . . . . . . . . . . . . . . . 129

4.3 Experimental results . . . . . . . . . . . . . . . . . . 133

4.4 Discussion . . . . . . . . . . . . . . . . . . . . . . . 135

4.5 Acknowledgement . . . . . . . . . . . . . . . . . . . . . . 139

4.6 Appendix* . . . . . . . . . . . . . . . . . . . . . 140

4.6.1 Nonlinear effects of restoring capillary pressure . . . . . . . 140

4.6.2 Analytic frequency amplitude approach . . . . . . . . . . 140

5 Apparatus and Experimental Procedure 142

5.1 Overview . . . . . . . . . . . . . . . . . . . . . . . . . . . . 142

5.2 Superstructure modification . . . . . . . . . . . . . . . . . . 142

5.3 Casting wheels . . . . . . . . . . . . . . . . . 143

5.3.1 Wheel hill-and-valley profiles . . . . . . . . . . . . . . 144

5.3 .2 Wheel deformation . . . . . . . . . . . . . . . 147

5.4 Data acquisition system . . . . . . . . . . . . . 150

5.5 Data measurement . . . . . . . . . . . . . . . . 151

5.6 Laser operation . . . . . . . . . . . . . . . . . . . . . . 154

5.7 Common experimental problems . . . . . . . . . . . . 158

6 Future Work 161

6.1 Onset of cross-wave defect . . . . . . . . . . . . . . . . . 161

6.2 Herringbone defect . . . . . . . . . . . . . . . . . . 163

6.3 Streak formation . . . . . . . . . . . . . . . 166

6.4 Puddle length and thickness measurements . . . . . . . . 176

A Dynamics of a Liquid Confined Between Two Plates 180

A.1 Overview . . . . . . . . . . . . . . . . . . . . 180

A.2 Geometric formulation . . . . . . . . . . . . 182

A.3 System dynamics . . . . . . . . . . . . . . . . . . . . . . . 184

A.3.1 Analytic method . . . . . . . . . . . . . 185

A.3.2 Higher order corrections . . . . . . . . . . . . . . 187

A.4 Comparison to cross-wave defect frequency . . . . . . . . . . . 191

$\begin{array}{ll}\text { B Experimental Data } & 194\end{array}$ 


\section{LIST OF TABLES}

1.1 Process parameters with typical values . . . . . . . . . . 3

1.2 Material properties (aluminum unless otherwise noted) . . . . . . 4

1.3 Relevant dimensionless groups for the fluid flow in the PFMS process 4

1.4 Properties of the copper $-0.4 \%$ beryllium casting wheels . . . . . 5

1.5 Summary of the time scales relevant to the PFMS process . . . . 26

3.1 Summary of $f_{1}, f_{2}$ and average cross-stream wave frequency for different $G$ values. . . . . . . . . . . . . . . . . . . . . . . 92

3.2 Behavior of frequency $f_{1}$ as the wheel speed $U$ is varied. . . . . . . 95

3.3 Cast-averaged data and control parameters for data appearing in the paper. . . . . . . . . . . . . . . . . . 121

5.1 Summary of casting wheels and properties of each. . . . . . . . . 144

B.1 Operating conditions and measured ribbon thickness from casting experiments Summer 2006 - Spring 2002. . . . . . . . . . . 194 


\section{LIST OF FIGURES}

1.1 (a) Schematic of the PFMS apparatus. b) Blow-up of the contact zone or puddle region. . . . . . . . . . . . . . . . 2

1.2 Security tags consist of layers of melt-spun ribbons which can be magnetized or demagnetized and detected by sensors. . . . . . . . . 10

1.3 Summary of the thickness variations at each relevant length scale for the PFMS process. . . . . . . . . . . . . . . . 26

1.4 Thickness variations occur over the length of the cast. . . . . . . . 28

1.5 Gap height as a function of time . . . . . . . . . . . . . . . . 29

1.6 Puddle length, $L$, variations occur over the length of the cast. . . . 30

1.7 Photograph of cross-stream wave as seen on $(a)$ free side and $(b)$ substrate side of ribbon product. . . . . . . . . . . . . . . 32

1.8 Model problems considered where the classical droplet vibrations are compared with the cross-wave defect. . . . . . . . . . . . 34

2.1 Schematic of the nozzle superimposed on images of the puddle from high speed video. . . . . . . . . . . . . . . . . . 43

2.2 Experimental data showing the time dependent behavior of the cast. 44

2.3 Fast fourier transform of puddle length, $L$, data showing dominant frequency at $5 \mathrm{~Hz}$, corresponding to the frequency of the wheel rotation. . . . . . . . . . . . . . . 4 46

2.4 Control volumes for the solid and liquid areas of the contact zone along with their geometric definitions. . . . . . . . . . . . . . 47

2.5 Control volume for the heat transfer that is used to calculate the temperature rise and expansion of the wheel. . . . . . . . . . . 50

2.6 The calculation for the time dependent behavior of $G$. . . . . . . . 53

2.7 Percent deviation from steady state. . . . . . . . . . . . . 55

2.8 Predictions for $T$ and $L$ based on the steady state mass balances and Bernoulli flow. . . . . . . . . . . . . . . . 58

2.9 Average solidification velocity through a cast. . . . . . . . . . . 60

2.10 Experimental and predicted data showing the time dependent behavior of the cast. . . . . . . . . . . . . . . . 62

2.11 Images of the USM and wheel surface at different stages of the cast. 67

2.12 Predicted values for $\mathrm{T}(\mathrm{t}), \mathrm{L}(\mathrm{t})$ and $\Theta(t)$ using the measured value

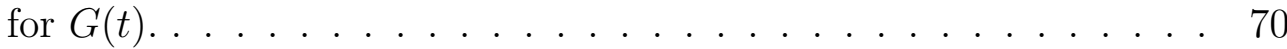

2.13 Experimental data from cast ODSU06-22. . . . . . . . . . . . . . 72

2.14 Experimental data from cast TBSP06-20. . . . . . . . . . . . . . 73

2.15 Experimental data from cast ODSU06-42. . . . . . . . . . . . 74

2.16 Experimental data from cast ODSU06-43. . . . . . . . . . . . . 75

2.17 Experimental data from cast ODSU06-47. . . . . . . . . . . 76

3.1 Profile of ribbon surfaces (a) Scan of the free-side showing two cross-waves. (b) Scan of substrate-side. . . . . . . . . . . . . 80 
3.2 Evolution of (a) ribbon thickness, (b) defect wavelength and (c) gap spacing through a cast with $\Delta P$ held constant. . . . . . . .

3.3 Types of puddle motions. $A_{1}$ is a large scale left-right motion of the USM, while $A_{2}$ is a smaller scale left-right vibration of the USM. 88

3.4 Images of the USM motion over 1 period of oscillation. . . . . . . . 89

3.5 Times Series of USM motion (type $A$ ) for $G=1.3 \mathrm{~mm}$. . . . . . 91

3.6 Schematic of the interface deviation during the USM vibration. . . 93

3.7 The interface deviation from the average USM shape, showing two nodes in the motion. . . . . . . . . . . . . . . . . . . 93

3.8 Point-wise data. Progression with time is from left to right within each of the three data sets. . . . . . . . . . . . . . . . 97

3.9 Predicted wavelength compared with experimental values. . . . . . 98

3.10 Geometry of model problem of capillary vibrations in a 2D channel. 100

3.11 BN spray applied to wheel in a straight pattern. The cast direction is from left to right. . . . . . . . . . . . . . . 107

3.12 Image of ribbon with visible marks from the straight BN strips on both the wheel and air side of the sample. The cast direction is from left to right. . . . . . . . . . . . . . . . . . . 109

3.13 Profile of the ribbon surfaces. (a) Scan of the free-side showing the cross-wave defect. (b) Scan of free-side showing the effect of the BN droplets. . . . . . . . . . . . . . . . . . . 110

3.14 Profile of the ribbon surfaces. (a) Scan of the substrate-side showing the cross-wave defect. (b) Scan of substrate-side showing the effect of the BN droplets. . . . . . . . . . . . . . . . . . . 111

3.15 (a) Puddle position during low overpressure casts. (b) Puddle positions during nominally pure $\mathrm{Al}$ casts. . . . . . . . . . . . . . . 113

3.16 Cross-stream defect frequency plotted against capillary frequency, without accounting for substrate out-of-roundness . . . . . . . . 120

4.1 Pinning along a circle-of-contact (dotted line) varies in position. . . 124

4.2 Schematic of coupled droplets pinned at tube-ends of radius $r$. $V_{1}$

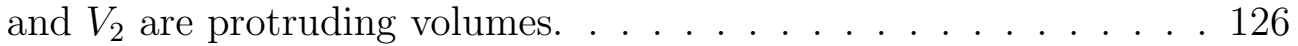

4.3 Bifurcation diagram with typical equilibrium shapes sketched. . . . 130

4.4 a) Dimensional vibration frequency according to linear theory (solid line) and compared to experiment (symbols). (b) Frequency of oscillation for limit cycles of amplitude $\Theta$ scaled by the linear frequency. 132

4.5 Experimental setup for droplet system with a steady pressure bias in the lower chamber and transient applied pressure pulse in the upper chamber. . . . . . . . . . . . . . . . . . . 134

4.6 Phase-plane plots of experimental data as single frames (dots) and as time-averages over many frames (solid curves) . . . . . . . . 135

4.7 Plot of pressure difference, $C(\Theta)$, vs $\Theta$ for various values of $\lambda$. . 141

5.1 Redesign of the experimental caster used in Olin Hall. . . . . . . 143 
5.2 Profile of the large wheel showing the out-of-roundness with three peaks and valleys. . . . . . . . . . . . . . . . . 145

5.3 Profile of the small wheel showing the out-of-roundness with one peak and valley. . . . . . . . . . . . . . 146

5.4 Profile comparison of the center of the three wheels showing the out-of-roundness of each. . . . . . . . . . . . . . . 147

5.5 Gap height and thickness measurements through the cast. . . . . 149

5.6 Run-out measurement of the gap height through the cast showing that the small wheel as it thermally expands and deforms. . . . . 150

5.7 Data acquisition system implemented through Labview. . . . . . 152

5.8 The gap sensor probe that measures voltage into an amplification box and is read by the data acquisition card. . . . . . . . . . 152

5.9 High frequency pulse laser used to template the substrate. . . . . . 154

5.10 Optical train used to focus laser to a point . . . . . . . . . 155

5.11 High frequency pulse laser focused with an optical lens and redirected to the substrate with a prism. . . . . . . . . 156

5.12 Focusing the laser on the meniscus directly leads to a finger growing from the puddle towards the laser. . . . . . . . . . . 156

5.13 The pulse laser is mounted on the 3 stage translational table. . . . 157

5.14 SiHub software interface used to position the laser. . . . . . . . 158

6.1 The connection between the meniscus motions and air capture is still not fully understood (shaded arrow). . . . . . . . . . . 162

6.2 Nozzles with varied geometries were used in an attempt to alter the flow in the puddle. . . . . . . . . . . . . . . . . 164

6.3 The herringbone defect where a) shows the wheel side and b) shows the air side. . . . . . . . . . . . . . . . . . 165

6.4 a) The herringbone defect occurs when the puddle is pinned at the nozzle slot. b) The puddle is free to move when the cross-wave defect occurs. . . . . . . . . . . . . . . . . 165

6.5 The herringbone defect frequency plotted against the capillary time scale. . . . . . . . . . . . . . . . . . . . . 167

6.6 Under very low overpressure conditions, the puddle is pinned at the slot and does not respond to the gap forcing. . . . . . . . . 168

6.7 Schematic of the longitudinal streaks that begin to appear in the ribbon as the cast progresses. . . . . . . . . . . . . . . . . 169

6.8 Streaks on the wheel side of the ribbon begin to appear in the ribbon as the cast progresses. . . . . . . . . . . . . 170

6.9 Streaks also appear on the air side of the ribbon as the cast progresses.171

6.10 Operating window for the PFMS process indicating the limits for successful casting. [From (Steen \& Karcher, 1997)] . . . . . . . . 172

6.11 Operating window using through cast data indicating where each cast transitions to the streak defect. . . . . . . . . . . 173 
6.12 Plot of We vs Wheel Temperature showing how the onset of the streaking corresponds to the wheel temperature. . . . . . . . . 174

6.13 Plot of PI vs Wheel Temperature showing how the onset of the streaking corresponds to the wheel temperature. . . . . . . . . . 175

6.14 Through-cast measurements of T and L for cast TBSP06 20. . . . . 176

6.15 Through-cast measurements of $\mathrm{T}$ and L for cast TBSP06 65. . . . . 177

6.16 Through-cast measurements of T and L for cast ODSU06 22. . . . 177

6.17 Through-cast measurements of $\mathrm{T}$ and L for cast ODSU06 40. . . . 178

6.18 Through-cast measurements of T and L for cast ODSU06 42. . . . 178

6.19 Through-cast measurements of $\mathrm{T}$ and L for cast ODSU06 43. . . . 179

6.20 Through-cast measurements of T and L for cast ODSU06 47. . . . 179

A.1 Coupled droplet confined between two plates. . . . . . . . . . . . . 180

A.2 Schematic of 2D fluid confined between two parallel plates: a) Corresponds to an equilibrium configuration while b) corresponds to a disturbed state. . . . . . . . . . . . . . . . . . . 181

A.3 Geometry of coupled droplet system confined between two plates. . 181

A.4 Disturbances of $\alpha$ and $\beta$ (around equilibrium value $\left(\alpha_{0}=\beta_{0}=\pi / 4\right.$ ) that satisfy constant volume constraint. . . . . . . . . . . . . 185

A.5 Vibration frequency vs equilibrium angle for various aspect ratios. . 188

A.6 Frequency-amplitude relation for $\alpha_{0}=\pi / 4$ as the aspect ratio $L$ is varied. . . . . . . . . . . . . . . . . . . . 191

A.7 Frequency-amplitude relation as $\alpha_{0}$ is varied and the aspect ratio is fixed at $L=10$. . . . . . . . . . . . . . . . . . . . . . . . . . 192

A.8 Vibration constant as the cast puddle aspect ratio is varied, for various curvature angles. . . . . . . . . . . . . . . . . . . 193 


\section{Chapter 1}

\section{Planar-flow Melt Spinning and Capillary Vibrations Background}

\section{$1.1 \quad$ Introduction}

Planar-flow melt spinning (PFMS, alternatively known as single roll spin casting or planar flow spin casting) is a continuous casting method for producing thin sheets or ribbons of metal. A schematic of the overall process is shown in Figure 1.1 a). Molten metal flows onto a moving wheel substrate where it solidifies and 'spins' off as a continuous ribbon. The industrial motivation for PFMS comes from the material properties of the cast product, as well as the cost savings benefit of processing in a single stage (Pimputkar et al., 1985). Properties result from the processing which occurs within the dynamics of the contact zone, as shown in Figure $1.1 \mathrm{~b}$ ). Molten metal is fed through a nozzle onto the moving substrate and a molten metal puddle is formed. Table 1.1 gives the dimensions of the nozzle and the range of operating parameters for the variables given in Figure 1.1. Table 1.2 lists the material properties of aluminum (typical casting material). Table 1.3 lists the relevant dimensionless groups for the PFMS process and Table 1.4 gives the properties of the casting wheel. The complicated interaction between fluid flow, heat transfer, solidification are subject to active research. The improvement of the quality of the cast product is of interest industrially. However, achieving an improved ribbon requires a fundamental understanding of the underlying physics of the contact zone.

The process, shown in Figure 1.1, feeds molten metal from a crucible (due 


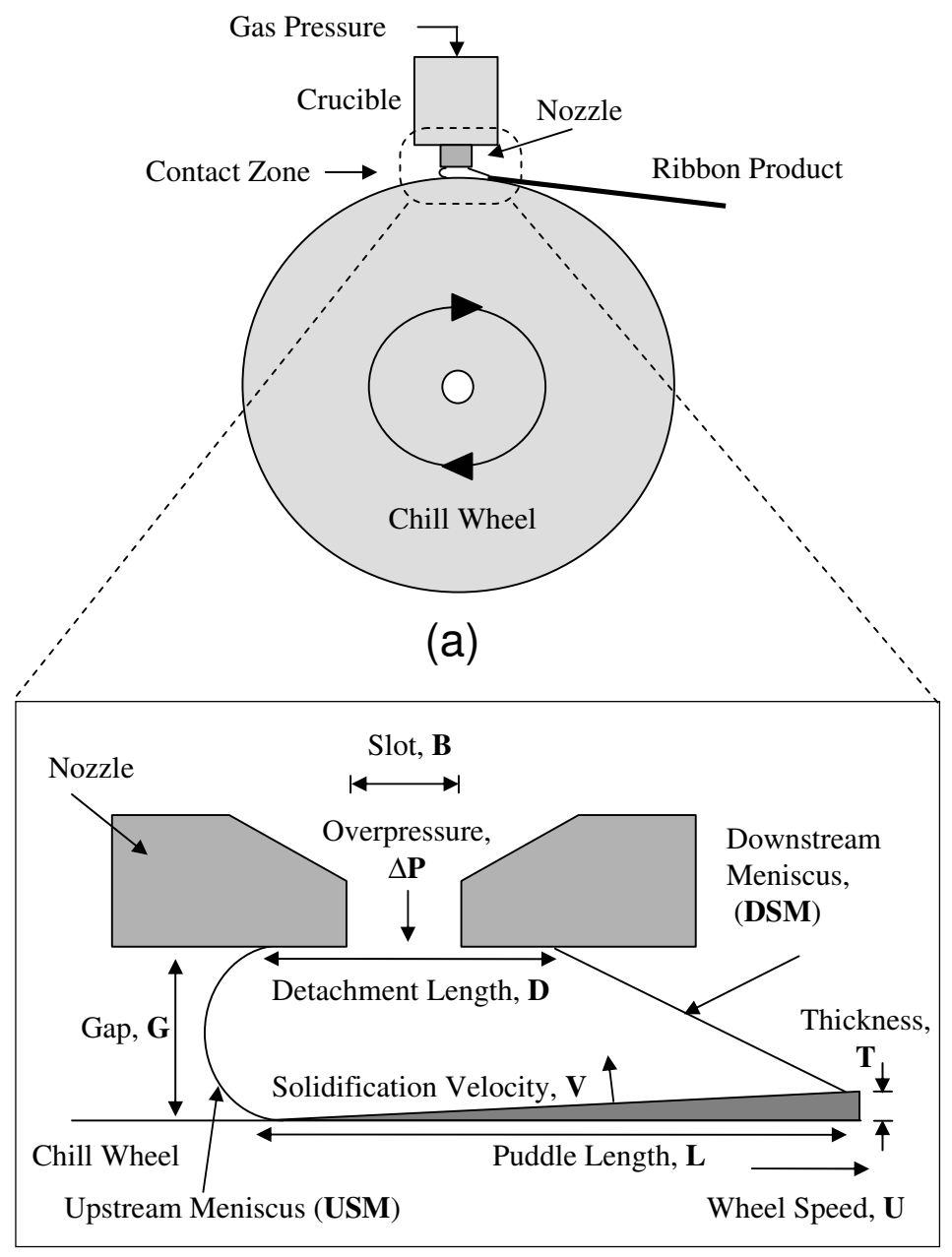

(b)

Figure 1.1: (a) Schematic of the PFMS apparatus. Molten metal flows from the crucible through a nozzle onto the spinning substrate or chill wheel, where it forms a puddle. Solidification occurs and a continuous ribbon is 'spun' from the puddle and released from the substrate. (b) Blow-up of the contact zone or puddle region. Note that the horizontal length scale has been compressed for clarity. 
Table 1.1: Process parameters with typical values

\begin{tabular}{lll}
\hline Nozzle geometry & \\
$\mathrm{G}$ & $0.5-1.3 \mathrm{~mm}$ & range of nozzle/wheel gaps \\
$\mathrm{B}$ & $1.6-3.2 \mathrm{~mm}$ & nozzle slot breadth \\
$\mathrm{W}$ & $2.5-5 \mathrm{~cm}$ & nozzle slot width \\
& & \\
Process variables & \\
$T$ & $0.08-0.35 \mathrm{~mm}$ & range of ribbon thickness \\
$L$ & $4-20 \mathrm{~mm}$ & typical range of puddle length \\
$D$ & $2-10 \mathrm{~mm}$ & typical range of detachment length \\
$L_{R}$ & $1-25 \mathrm{~cm}$ & ribbon length over which $T$ is averaged \\
$U$ & $5-15 \mathrm{~ms}^{-1}$ & wheel speed (linear) \\
$M$ & $500-1200 \mathrm{~kg}^{2}$ & mass of molten metal \\
$\Delta P$ & $3-6 \times 10^{3} \mathrm{Nm}^{-2}$ & overpressure \\
$V$ & $0.05-0.15 \mathrm{~ms}^{-1}$ & solidification rate \\
$\Theta$ & $30^{\circ} \mathrm{C}$ & initial wheel temperature \\
$\Delta \Theta$ & $30-100^{\circ} \mathrm{C}$ & wheel heat-up \\
$T_{h}$ & $100{ }^{\circ} \mathrm{C}$ & melt superheat \\
\hline
\end{tabular}


Table 1.2: Material properties (aluminum unless otherwise noted)

\begin{tabular}{lll}
\hline$\rho_{s}, \rho_{l}$ & $2.7 \times 10^{3}, 2.3 \times 10^{3} \mathrm{kgm}^{-3}$ & solid, liquid density \\
$k_{s}, k_{l}$ & $229,103 \mathrm{Jm}^{-1} \mathrm{~s}^{-1} \mathrm{~K}^{-1}$ & solid, liquid thermal conductivity \\
$C_{p, s}, C_{p, l}$ & $938,1080 \mathrm{Jkg}^{-1} \mathrm{~s}^{-1}$ & solid, liquid specific heats \\
$\eta$ & $10^{-3} \mathrm{kgm}^{-1} \mathrm{~s}^{-1}$ & viscosity $(1033 \mathrm{~K})$ \\
$\sigma$ & $0.86 \mathrm{Nm}^{-1}$ & surface tension $(1033 \mathrm{~K})$ \\
$T_{m}$ & $933 \mathrm{~K}$ & melting temperature \\
$\Delta \nu$ & $3.87 \times 10^{5} \mathrm{Jkg}^{-1}$ & enthalpy of formation $(933 \mathrm{~K})$ \\
\hline
\end{tabular}

Table 1.3: Relevant dimensionless groups for the fluid flow in the PFMS process

\begin{tabular}{|c|c|c|}
\hline Re & $10^{4}$ & Reynolds $\#=\rho U G / \mu$ \\
\hline$W e$ & $10^{2}$ & Weber $\#=\rho U^{2} /(2 \sigma / G)$ \\
\hline$P I$ & $10^{0}$ & Pressure index $=\Delta P /(2 \sigma / G)$ \\
\hline$C a$ & $10^{-3}$ & Capillary $\#=\mu U / \sigma$ \\
\hline Bo & $10^{-2}$ & Bond $\#=\rho g G^{2} / \sigma$ \\
\hline
\end{tabular}


Table 1.4: Properties of the copper $-0.4 \%$ beryllium casting wheels

Wheel geometry

$R_{w} \quad 30$ or $45 \mathrm{~cm} \quad$ radius of wheel

$C_{w} \quad 191$ or $283 \mathrm{~cm} \quad$ circumference of wheel

$W_{D} \quad 1.27 \mathrm{~cm} \quad$ wheel depth

$W_{W} \quad 13.5 \mathrm{~cm} \quad$ width of wheel

Physical properties of the wheel

\begin{tabular}{lll}
$\rho_{w}$ & $8.96 \times 10^{3} \mathrm{kgm}^{-3}$ & wheel density \\
$k_{w}$ & $4.0 \mathrm{Jm}^{-1} \mathrm{~s}^{-1} \mathrm{~K}^{-1}$ & thermal conductivity of wheel \\
$C_{p, w}$ & $385 \mathrm{Jkg}^{-1} \mathrm{~s}^{-1}$ & specific heat of wheel \\
$\alpha$ & $16.5 \times 10^{-6} \mathrm{C}^{-1}$ & coefficient of thermal expansion \\
$S_{R}$ & 150 grit sand paper & surface roughness \\
\hline
\end{tabular}


to imposed overpressure $\Delta P$ ) through a nozzle of size $B$ and into a narrow gap region between the nozzle and chill wheel (or substrate). The nozzle width $W$ can be controlled to provide for casting of wider products. Because of the small gap height, $G$, the nozzle interferes with the flow, and a puddle held by surface tension is formed. $\Delta P$ is held constant during a cast and $G$ adds hydrodynamic resistance to the flow rate. (Since $G$ influences the flow of molten metal the PFMS process is a 'self-metered' process. The situation is changed if the flow rate through the nozzle is fixed, in which case it would be a 'pre-metered' process.) The close proximity of the nozzle to the substrate tends to stabilize the puddle. The contact between the wheel and molten puddle is such that enough heat is removed from the melt for solidification to occur. The solidification front moves at an average velocity, $V$. The substrate (moving with linear velocity $U$ ) continually removes the solidified product from the casting zone.

The current study focuses mainly on the time dependent behavior of the process, with particular emphasis on the dynamics of the molten metal puddle. The observed puddle motions have led to a more general study of capillary droplet vibrations. Section 1.2 gives a background on the development of the PFMS process and the applications of rapidly solidified products. Section 1.3 gives a literature review of the work that is relevant to the current study. Section 1.4 gives a summary of the results of this study. A discussion of the time dependent behavior of the cast is given over the longest length scales (the entire $50 \mathrm{~m}$ cast length), down to the microstructural length scale $(\mu m)$. Surface defects occur on an intermediate scale $(\mathrm{cm}$ to $\mathrm{mm})$ and can correspond to vibrations of the molten metal puddle. These puddle motions are compared to the classical problem of capillary / inertia vibrations of a liquid droplet. 


\subsection{Background}

Continuous casting of metals in a single stage has been envisioned for over a century, dating back to the initial patent by Bessemer, 1865. There are a number of casting methods which can be used to produce continuous ribbons, such as the twin roll casting method used in the production of steel slabs (e.g. Meng \& Thomas, 2003). The ribbons can be post-processed to further reduce the thickness, e.g. rolling stages to produce aluminum foil, but the additional stages are often costly and lead to additional environmental concerns. The PFMS process is the preferred method for manufacturing wide $(>m m)$ sheets of foil with a thickness less than $\approx 100 \mu \mathrm{m}$

The cost savings benefit of processing in a single stage is one of the two motivations to use the PFMS process. The novel microstructure formations during processing lead to a new category of specialty metals. The rapid quenching of molten metal to foil can result in amorphous and micro-crystalline structures (Duwez et al., 1960). Cooling rates on the order of $10^{6} \mathrm{~K} / \mathrm{s}$ are observed in the PFMS process, which can prevent the grain structures that are typical of slowly cooled alloys. These novel materials were the initial motivation for research into rapid solidification. A review of the history of rapid solidification processing is given by Jones, 1984. Only within the past few decades has continuous casting of amorphous foils been realized. There has been a great deal of interest in the PFMS process since its initial patent for the production of amorphous foils (Narasimhan, 1979).

Splat drop quenching and free jet melt spinning (FJMS, also known as chill block casting) are alternative processes for casting rapidly solidified materials. Splat quenching involves shooting small droplets of metal onto a substrate where they rapidly solidify. Overviews of the various techniques used for splat drop 
quenching are available (Ruhl, 1967; Motojima et al., 1979). FJMS involves a continuous liquid jet of metal impinging onto a moving substrate. A droplet shaped molten metal 'pool' forms, from which a continuous ribbon is removed. There is no hydrodynamic interaction between the flow of the liquid jet and the molten metal pool (i.e. FJMS is a pre-metered process). An overview of the free jet process is given by Kavesh, 1978. Both processes are capable of casting amorphous material, however, splat quenching is a batch process and the cast material from FJMS is of limited width. The size of the molten metal pool determines the width of the ribbon cast in the FJMS process. Thickness variations in the width direction are also commonly observed (Anthony \& Cline, 1978, 1979; Napolitano \& Meco, 2004). These thickness variations correspond to the 'natural' modes of vibration of the molten metal pool. The small width and the poor quality of the FJMS materials has limited potential applications to small scale electrical devices (Hasegawa, 1983).

The development of PFMS in the early 1980s began to address the quality and width issues during production. The low capital cost for experimental PFMS casters, in part, helped the growth of research into rapidly solidified material (Smith \& Saletore, 1986). During the processing, molten metal flows through a nozzle of width, $W$, onto a moving substrate where the ribbon is formed. The nozzle geometry determines the width of the ribbon. The amount of cast material (the 'charge') in many experimental studies is less than a kilogram, limited by the size of the crucible which contains the molten metal (Huang, 1981; Yukumoto et al., 1985; Wilde \& Matthys, 1992; Praisner et al., 1995). Active cooling of the substrate is typically not applied for such small charges.

Large scale production of continuous ribbons requires a constant feed of material and active cooling of the substrate. The production of transformer cores using 
the PFMS process was one of the initial applications for amorphous foils (Belden, 1985). It was found that rapidly quenched ferromagnetic alloys have low magnetic permeabilities, leading to lower power loses than conventional silicon steel based cores (Hasegawa, 2001). The improvement in transformer performance helps to reduce the emission of greenhouse gases. In addition to the reduced electrical losses, amorphous based cores reduce transformer size and reduce noise (Hasegawa, 2004).

The applications of PFMS materials are growing as the need for specialty materials increases. Security tags, used by many retail stores, are a common product that uses melt-spun ribbons. An opened security tag is shown in Figure 1.2, revealing the layers of melt-spun ribbons. The magnetized or demagnetized ribbons are detected by sensors. There is currently a wide range of applications for rapidly solidified products (Jones, 2001), and specifically amorphous aluminum (Perepezko \& Hebert, 2002; Katgerman \& Dom, 2004). However, the fundamental physics of the PFMS process are not well understood. Defect formation in the foil is still problematic, and rarely is the defect formation mechanism identified. To that extent, PFMS is still somewhat of an art form rather than a science.

The geometry of the PFMS caster is similar to many coating processes, such as slot coating. A recent review discusses the dynamics of coating flows (Weinstein \& Ruschak, 2004). The processing speeds for the needed cooling rates of the PFMS process are much higher than typical ranges for traditional coating flows, however. Also, the properties of molten metals are significantly different from most coating materials, such as viscous fluids and polymers. Dynamic wetting, even in well established fields, is still largely empirical in nature (Blake, 2006). Dynamic wetting in the PFMS process is additionally complicated by large temperature gradients and the solidifying metal. Further research is still needed to understand 


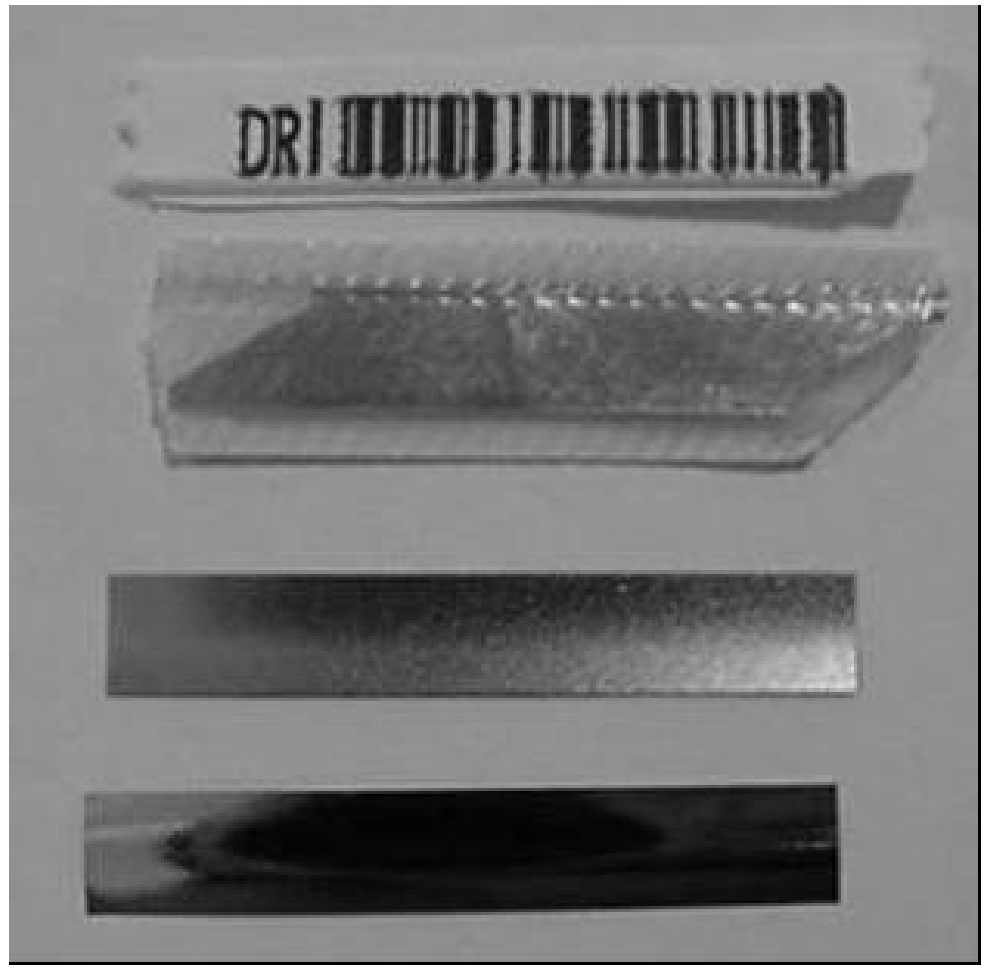

Figure 1.2: Image of a common security tag found in retail products. The security tag has been opened revealing the layers of melt-spun ribbons which can be magnetized or demagnetized and detected by sensors. 
the interactions of fluid flow, high speed contacting dynamics, heat transfer and solidification that occur in the PFMS process.

\subsection{Literature review}

This section provides a review of the previous works which are relevant to the current study. The experimental conditions for PFMS process can vary greatly depending on the casting process parameters, often making direct comparisons between published results difficult. The experimental parameter space leads to many possible dimensionless groups which may be relevant to the final properties of the ribbon. We have tried to fix as many operating conditions constant as possible and focus on the relating the ribbon thickness, $T$, to the variables $B, G$, $\Delta P$, and $U$. The initial wheel temperature $\left(30^{\circ} C\right)$, wheel roughness (the wheel is sanded with 150 grit paper), melt superheat $\left(100^{\circ} \mathrm{C}\right)$ and melt composition (Al$7 \% \mathrm{Si}$ ) are generally held constant.

Section 1.3.1 discusses the steady state behavior of the PFMS process, which relates the processing parameters to the average $T$. Section 1.3 .2 addresses the unsteady aspects of the process, focusing on how the molten metal puddle evolves

in time. Defect formation in the ribbon is often attributed to unsteady features of the puddle menisci or contact lines. Motions of the puddle menisci are similar to the classical capillary vibrations of a 'plucked' sphere. Finally, Section 1.3.3 gives an overview of droplet oscillations, putting the molten metal puddle motions into the more general context of liquid droplet vibrations. 


\subsubsection{Steady state behavior}

Much of the work in PFMS has focused on the material properties of the cast product. Initial studies focused on the heat transfer aspects of the process and identified the operating conditions which resulted in amorphous material (Kavesh, 1978; Jones, 1982). Other studies focused on the steady state behavior of the process, relating the processing parameters to the ribbon thickness, $T$. The puddle length, $L$, combined with $U$ sets the time which the molten metal is in contact with the wheel (residence time, $\tau_{L}=L / U$ ). Carpenter \& Steen, 1997 give the limiting cases for growth of the solidifying ribbon as either conduction-limited $\left(T \propto \tau_{L}\right)$ or contact-limited $\left(T \propto \tau_{L}^{1 / 2}\right)$. It is generally found that $T \propto U^{-A}$, with reported values ranging from $A=1$ ( Fiedler et al., 1984; Ibaraki \& Steen, 1996) to $A=2 / 3$ ( Carpenter \& Steen, 1992; Praisner et al., 1995). This correlation indicates that the solidification front within the puddle is typically closer to the conduction limited case.

The ribbon evolution depends strongly on the heat transfer from the molten metal puddle to the wheel. The contact-averaged heat transfer coefficient, $h_{i}$, has been reported to be in the range of $10^{4}$ to $10^{6} \mathrm{Wm}^{-2} \mathrm{~K}^{-1}$ (Huang \& Fiedler, 1981; Kukura et al., 1995). This value is specific to each experimental setup and depends on the processing parameters, as well as the conditions of the wheel. The steady state mass balance around the solidifying ribbon gives $L V=T U$ as a balance between the 'freeze' rate $L V$ and the 'pull rate' $T U$. The reported values of $h_{i}$ are based on combining $L$ measurements during the cast and the $1 \mathrm{D}$ solution of the heat equation, as discussed in Carpenter \& Steen, 1997; Ibaraki, 1996. Other methods for determining $h_{i}$ include photocalorimetric measurements of the ribbon 
or by estimating the cooling rate from measurements of the secondary dendrite arm spacing of the ribbon microstructure (Davies, 1985).

The fluid flow dynamics of the process can often be treated separately from the heat transfer. It has been found that for long liquid puddles, the energy equation decouples from the momentum equation, allowing for heat transfer to be solved for independently from the fluid dynamics of the PFMS process (Carpenter \& Steen, 1997). The columnar grain growth that is observed in microcrystalline material is generally perpendicular to the substrate and tends to be in the direction of the highest temperature gradient. However, it has been noted that the columnar structures appear slightly inclined from normal, indicating a weak coupling between the fluid flow and heat transfer (Emmerich, 1985).

Table 1.3 lists the dimensionless groups which are relevant to the fluid dynamics of the process. The magnitude of the Reynolds number $(R e)$ indicates that inertia is dominant over viscous forces in the puddle. The capillary number $(\mathrm{Ca})$ and Bond number $(B o)$ show that capillary forces are more significant than viscous or gravitational forces. The pressure index $(P I)$ is $O(1)$, consistent with the capillary pressure at the upstream meniscus balancing the overpressure, $\Delta P$, that sets the flow through the nozzle. The Weber number $(W e)$ appears to be $O\left(10^{2}\right)$, based on the characteristic speed $U$. It is more appropriate, however, to use the average flow rate in the puddle, $\bar{u}$, for this group. Although $\bar{u}$ is not measured directly, scaling estimates give $\bar{u}=T U / G \approx U / 10$. We then becomes $O(1)$, consistent with inertia and capillary forces balancing in the puddle.

The flow in the puddle region is largely inviscid and has been modeled analytically by combining mass and Bernoulli balances, as formulated by Huang, 1981. This analytic model has been used in a recent experimental work to relate $T$ to 
the operating parameters (Tkatch et al., 2002). A review of the fluid mechanics of the PFMS process is given by Steen \& Karcher, 1997. Assuming that molten metal is an ideal incompressible fluid, the flow rate leaving the nozzle is given as $u_{\text {in }}=\left(2 \Delta P / \rho_{l}\right)^{1 / 2}$, where $\rho_{l}$ is the liquid density. The continuity equation gives $B u_{i n}=G \bar{u}=T U$, where $G$ is the gap height, and $U$ is the wheel speed. Combining these results gives the dimensionless scaling that predicts $(T / G)^{2} \propto \Delta P /\left(\rho_{l} U^{2}\right)$. The $\propto$ symbol is used because this scaling neglects physics such as gravity, dissipative losses in the flow, the density change upon solidification and the molten metal superheat. Subsequent work has resulted in the empirical fit given as

$$
\frac{T}{G}=a\left(\frac{\Delta P}{\rho_{l} U^{2}}\right)^{b},
$$

where $a$ and $b$ are determined from experimental data analysis (Carpenter \& Steen, 1992; Praisner, Chen \& Tseng, 1995; Ibaraki \& Steen, 1996). The results depend upon the nozzle contraction ratio, $B / G$, and indicate deviations from pure Bernoulli-type flow. An analytic model focused on characterizing the frictional loss as the flow turns the $90^{\circ}$ bend around the front edge of the nozzle has also been given (Sung et al., 1994).

Efforts have been made to computationally simulate the flow and temperature fields within the molten metal puddle. A brief discussion of this work will be given, although analytic modeling is the main focus of the current study. The free surfaces of the puddle and the solidification front complicate the numerical procedures. A constant flow rate through the nozzle is one of the more common assumptions used, even though most experimental casters use a constant overpressure in the crucible. There are many numerical studies which provide insight into how the 
puddle and ribbon thickness respond to changing processing variables, but there is a lack of experimental verification of these models.

An early numerical study solved the heat and momentum equations simultaneously using a fractional volume-of-fluid (VOF) method that preserves enthalpy (Wu et al., 1992). The solidification was modeled by the Vogel-Fulcher equation, which allows the viscosity of the molten metal to vary with temperature and approach infinity at the melting point. Latent heat is neglected by this formulation. The results predicted how puddle initially forms and how changing process parameters effect the fluid flow and heat transfer. A subsequent publication examined the 3D effects of the process (Chen \& Hwang, 1995).

A control-volume finite-difference method was introduced by Pryds \& Hattel, 1997, which accounted for the temperature dependent thermophysical parameters, nucleation and recalescence effects. Interestingly, it was shown that the interface temperature of the wheel increases rapidly while under the puddle and should not be neglected. The heat transfer is sensitive to the magnitude of the heat transfer coefficient, $h_{i}$. Increasing $h_{i}$ results in a higher wheel surface temperature. However, varying the initial wheel temperature over a range of $200{ }^{\circ} \mathrm{C}$ had little effect on the ribbon thickness. Increasing the melt superheat resulted in a higher undercooling and delayed the time needed for nucleation. Once nucleation occurred, however, the time for solidification to finish was decreased.

A more recent study focused on the steady flow and temperature fields within the puddle, but also included wetting effects of the puddle (Bussmann et al., 2002). Steady fluid recirculation zones in the upstream and downstream regions of the puddle are identified, as is the pressure profile along the length of the puddle. The flow and temperature streamlines are given. The contact angle between the puddle 
and the wheel is prescribed as a function of the contact line speed. The upstream and downstream contact angles between the puddle and the nozzle are assumed to be equal and are input parameters for the numerical model. It is predicted that the contact angles along the nozzle play a significant role in determining the flow streamlines, while the contact angle along the moving wheel does not. This is somewhat surprising considering the complicated dynamics of the moving contact line. Including wetting effects is an improvement over previous models, although there are still questions of the model relevance. The experimental casting uses constant applied pressure, not constant flow rate. Also, the assumption of a constant wheel temperature with a single value for the heat transfer coefficient is used. Finally, a comparison with experimental results have not been made.

\subsubsection{Time dependent behavior}

There are a number of transient influences that effect the quality of the ribbon during our experimental casting. Most theoretical studies focus on identifying the steady behavior of the process, rather than how disturbances influence the ribbon. Table 1.5 lists the relevant time scales for the PFMS process and can be used to separate the 'slow' and 'fast' variations in $T$. The out-of-roundness of the wheel and the heat-up of the wheel during the cast are correlated with variations in $T$, as discussed in Chapter 2. Both of these effects occur over the slow time scales of the entire cast time $\left(\tau_{\text {cast }}\right)$ and the substrate revolution time $\left(\tau_{\text {rev }}\right)$. A brief review of the related studies over these scales is given first. Variations in $G$ through the cast and the varying processing conditions which cause the heat transfer and contacting to change within a cast are addressed. Next, the fast $T$ variations, often referred to as defect formations, in the ribbon are discussed. These defects often correspond 
to small scale $T$ variations in the ribbon and occur over the time scales $\tau_{c a p}$ $\tau_{\text {herr }}$. The dynamics of the puddle menisci can lead to small scale casting defects, as discussed in Chapter 3. However, experimental studies which report defect formation are far more common than those which provide the detailed mechanism of defect formation.

Wheel out-of-roundness is observed in our experimental casters and has been reported elsewhere (Wilde \& Matthys, 1992). This results in an increased uncertainty in the reported values of $G$ and $T$. As mentioned in Section 1.3.1, the steady state thickness, $T$, is proportional to $G$. The time scale for $G$ to vary is much longer than the puddle residence time $\left(\tau_{r e v} \gg \tau_{L}\right)$. This allows us to separate the effects of the wheel out-of-roundness from other parameters which influence $T$.

The wheel heat-up also effects $T$. Active cooling is not typically applied to the wheel, unless large scale production of ribbon is desired. Experimental castings commonly produce between $10-1000 \mathrm{~g}$ of material, limiting the need for cooling of the wheel. However, the temperature rise of the substrate can result in a thermal expansion of the wheel, causing $G$ to decrease during a cast. Vogt \& Frommeyer, 1985, computationally calculate the thermal expansion when casting a $2 \mathrm{~cm}$ wide ribbon on a casting wheel of dimensions $R_{w}=20 \mathrm{~cm}, W_{W}=5 \mathrm{~cm}$ and $W_{D}=1 \mathrm{~cm}$. The wheel is cooled internally by specifying a constant coolant temperature and the heat transfer coefficient between the wheel and the coolant. The predicted result is that the wheel expands by $20 \mu \mathrm{m}$ during the 100 revolutions of the cast. This is one of the few studies that couples the wheel heat-up with the thermal expansion of the substrate and illustrates that the temporal changes of the wheel can be significant even with internal cooling. The model by Vogt \& Frommeyer, 1985, only solved the energy balances for the wheel and the ribbon. The change 
in $G$ did not influence the puddle size and $T$ was assumed to be constant. They focused on coupling the wheel temperature to the microstructural features of the ribbon.

Other studies have focused on how $T$ varies within a cast. Praisner et al., 1995, report a decrease in $T$ by $\approx 10 \%$ over $11 \mathrm{~m}$ of cast length. They attribute this to a decrease in the metallo-static head of metal during the cast, rather than the thermal expansion of the wheel. However, Kahn, 2000, using an applied pressure compensation to keep $\Delta P$ constant through the cast observed a steady decrease in $T$. It was concluded that the decrease in $G$ was responsible for the decreasing $T$ trend. As discussed further in Chapter 2, the decrease in $G$ through the cast causes a 'quasi-steady' decrease in $T$, such that Equation 1.1 can be used to predict $T$ using the changing value of $G$ through the cast.

The fluid flow alone can be used to predict $T$ by uncoupling the flow rate from the solidification. This assumes that all of the molten metal that flows onto the wheel is eventually solidified. Kahn, 2000, showed that our experimental caster is 'feed limited' rather than 'freeze limited', indicating that it is the fluid flow that determines $T$ rather than the average solidification rate, $V$. However, many of the microstructural properties of the ribbon are determine by how rapidly the metal is solidified. The puddle length, $L$, is required to infer $V$ from the steady mass balance (i.e. $V=T U / L)$. In summary, $T$ can be predicted from the fluid flow alone, based on Equation (1.1). However, $L$ depends on both the fluid dynamics and the heat transfer that occurs between the puddle and the wheel.

There are still open issues in how the processing parameters influence the contacting between the puddle and the wheel. This has a direct influence on the heat transfer and solidification rate. The heat transfer between the molten metal puddle 
and the wheel depends upon many factors, including (but is not limited to) the wheel temperature, the surface texture, the thermophysical properties of the wheel and the wettability of the molten metal on the wheel. The heat transfer coefficient, $h_{i}$, groups many of the unknown effects into one parameter. The changing thermal conditions of the substrate can lead to a variation in $h_{i}$. Many studies have been done to characterize the contact-averaged $h_{i}$, as discussed in Section 1.3.1. However, $h_{i}$ has also been reported to vary with the time of the contacting (Vogt, 1987; Li \& Thomas, 1996). A changing $h_{i}$ is attributed to the variation in contacting between the solidifying ribbon and the wheel. Loulou et al., 1999, provide a detailed mechanism for how the contacting changes between a solidifying ribbon and a solid substrate during the initial stages of solidification.

The variation of $h_{i}$ for rapid solidification processes is more commonly investigated by studying how a molten metal droplet solidifies on a plate. The temperature history of a molten metal droplet was measured using a fast-response pyrometer (Wang \& Matthys, 2002). Matching model calculations to the experimental results gives the time dependent value of $h_{i}$. The results show that $h_{i}$ is initially $10^{5} \mathrm{~W} / \mathrm{m}^{2} \mathrm{~K}$, but drops to $10^{4} \mathrm{~W} / \mathrm{m}^{2} \mathrm{~K}$ as the droplet goes through the phase transformation and becomes solid. Similar studies have also characterized the initial variation of $h_{i}$, as well as the dependence on parameters such as surface roughness and droplet superheat (Wang \& Qiu, 2002; Heichal \& Chandra, 2005; Heichal et al., 2005).

Gong et al., 1991, developed a full two-dimensional model of the fluid flow and heat transfer within the puddle using a semi-implicit finite difference technique. The effects of $U, G$ and $u_{i n}$ are examined numerically. Experimental results of $L$ were used to validate the heat transfer modeling. The contact-averaged values for 
$h_{i}$ ranged from $3 \times 10^{5}$ to $7 \times 10^{5} \mathrm{~W} / \mathrm{m}^{2} \mathrm{~K}$. L was found to decrease in length as the contact-averaged $h_{i}$ increases. Muhlbach et al., 1987, used infrared temperature measurements and secondary dendrite arm spacings to show how $h_{i}$ varies with the position of the puddle. It was found that $h_{i}$ decreases from $10^{8}$ to $10^{5} \mathrm{~W} / \mathrm{m}^{2} \mathrm{~K}$ over 1.5 milliseconds. These studies combined numerical modeling with experimental results and illustrate the difficulty in matching the two.

Wheel temperature and the roughness can significantly influence the contacting between the molten metal puddle and the wheel. Casting in a vacuum or with a highly polished wheel surface tends to increase $h_{i}$, as the tendency for air capture is reduced for either case (Davies, 1985; Huang \& Fiedler, 1981). However, the enhanced contact area between the ribbon and the wheel can lead to adhesion (or welding) and the ribbon may not release from the wheel (Robertson et al., 1985). Adhesion of the ribbon can cause dangerous experimental conditions where the ribbon wraps around the wheel. This phenomena is not well understood, however. Liebermann, 1984, argues that the ribbon adheres to the wheel at a critical temperature, which is consistent with increasing $h_{i}$ resulting in a higher wheel interface temperatures. Another model for adhesion is given by Maringer, 1988, where molten metal forms a bond to the wheel surface upon solidification. Thermal stresses upon the cooling of the ribbon and the heating of the wheel then break this bond and the ribbon is released. Presumably, improved contacting would result in a greater adhesion bond resulting in increased chance that the ribbon will stick to the wheel. It is not clear that these are mutually exclusive viewpoints. (A discussion of ribbon adhesion on our experimental caster is given in Section 5.3.1.)

Achieving specified material properties of the ribbon and the desired $T$ are 
required for many industrial applications. A uniform surface quality of the ribbon is also important for many applications (Robertson et al., 1985). Thickness variations occurring on the over $\mathrm{cm}$ to $\mathrm{mm}$ length scales are often detrimental to the quality of the ribbon and are commonly referred to as casting defects. More often than not, defect formation is reported as experimental observation but the defect formation mechanism is rarely identified. The steady state behavior of the process does not account for these defects.

Various surface defects have been reported and are generally attributed to instabilities of the puddle menisci. Experimental studies have categorized the defect formations in the ribbon as: i) dimpled, with a rough air side profile, ii) striated, with lengthwise groves on the air side of the ribbon, iii) wavy, with cross-stream patterns corresponding to localized thickness variations and iv) herringbone, with a wavy cross-stream pattern spaced very close together (Carpenter \& Steen, 1992; Praisner, Chen \& Tseng, 1995). A formation mechanism for each defect is speculated and the corresponding processing parameters are given. All of these defects have been observed in our experimental casting.

A distinct periodic surface pattern, occurring at a frequency of $\approx 5000 \mathrm{~Hz}$ in the cross-stream direction of the ribbon, was identified as a 'fish scale' defect (Huang \& Fiedler, 1981). The patterns corresponds to a line of small cavities on the wheel side of the ribbon, with larger depressions on the air side. The Steen group have reported the herringbone pattern which closely resembles the fish scale defect. (A discussion of the herringbone defect on our experimental caster is given in Section 6.2.) The onset conditions for the fish scale defect depends upon the surface roughness of the wheel. It is postulated that small air pockets are entrained 
into the puddle at the upstream contact line and form the defect pattern in the cross-stream direction.

Few studies have focused on correlating puddle motions with defect formations. This is in part due to the high frequency at which defects are reported to occur, making imaging of the puddle motions difficult. Surface waves on the downstream meniscus have been reported by Gong et al., 1991; Wilde \& Matthys, 1992; Kahn, 2000. The motions have not been, however, correlated with defects in the ribbon.

A more recent experimental study by Haga \& Suzuki, 2003b, focuses on modifying the nozzle geometry and injection angles to try to optimize the ribbon quality. Various defects are reported, but without imaging of the process. It is implied that flow instabilities cause the defect formations observed. Presumably, modifying the nozzle geometry changes the flow dynamics and prevents the instabilities in the flow from forming defects in the ribbon.

\subsubsection{Droplet vibrations}

It is found in this study that capillary waves on the puddle menisci correlate with the 'cross-wave' ribbon defect, as discussed in Chapter 3. The combination of low viscosity and high surface tension of molten metals result in the puddle menisci being susceptible to capillary vibrations, as noted by Kavesh, 1978. The geometry of the molten metal pool of the FJMS process more closely resembles a spherical droplet than PFMS. The melt pool vibrations in FJMS correspond to the 'natural' modes of vibration of a spherical droplet, as mentioned in Section 1.2. The molten metal puddle vibrations motivates the more generalized study of capillary droplet vibrations. This section gives a brief review of capillary / inertia vibrations of fluid droplets and provides motivation for the work in Chapter 4 . 
Liquid droplets tend to spherical shapes under the action of surface tension. The vibrations of a sphere is a classical example of the competition between liquid inertia and capillarity, as summarized by (Lamb, 1932; Lord Rayleigh, 1879). The frequency and mode shapes predicted by Rayleigh have been verified in experiment and their relevance is well-documented. Gravity tends to influence the droplet shape for a typical fluid, such as water, unless the droplet size is below the $m m$ scale.

Various techniques for studying droplet dynamics have been developed. Immersing a droplet in an immiscible, density matched fluid is one approach to counter gravitational effects. Trinh et al., 1982, provides an experimental study of small-amplitude droplet vibration modes for a droplet in an immiscible fluid. An acoustic vibration is used to excite the first few vibration modes. A subsequent experimental study examined large-amplitude droplet oscillations (Trinh \& Wang, 1982). A 'soft' nonlinearity, where the resonant frequency is observed to decrease with increasing disturbance amplitude, is reported. An increase in the decay rate of the droplet motions are also observed at larger disturbance amplitudes.

It is more common for droplets to be in contact with a solid boundary. Strani \& Sabetta, 1984, modeled the free vibrations of a droplet contained is a spherical bowl. The constraint imposed by the solid boundary activates a translational mode of vibration. The results are compared with the experimental work of Bisch et al., 1982. Pendant (or sessile) droplet vibration have also received much attention for their relevance to applications such as crystal growth and dynamic surface tension measurements (Basaran \& DePaoli, 1994; Wilkes \& Basaran, 1997).

More recently, the resonant frequency of a column of fluid in a capillary tube has been studied experimentally by Hilpert \& Miller, 1999. The confinement of 
the fluid by the capillary tube is significantly different from the solid boundary of a pendant drop. However, there are now two fluid-air menisci that can interact and multiple vibration modes are now allowed. Bian et al., 2003, also examined the oscillation frequencies of a liquid confined in a circular cylinder, by both experimental and theoretical methods. The lowest mode occurs for oscillations of the menisci that remain as spherical cap shapes with pinned contact lines.

Coupling two symmetric droplets by a tube is another example of a system with multiple fluid-air menisci. The shared pressure between the droplets leads to one droplet scavenging volume from the other, breaking the symmetry. The resulting configuration has one super-hemispherical and one sub-hemispherical droplet (other equal curvature) connected by the tube. The two pressure-coupled droplets have a double-welled surface energy landscape. Motivation for studying the dynamics of this type of system comes from practical application. With a mechanism to trigger from one well to another, such as with an electro-osmotic pump placed in the tube (Vogel et al., 2005), the system becomes active. A number of applications that exploit capillary bi-stability are under development. These include optical micro-lens devices (Lopez et al., 2005). For these, understanding the dynamics of the droplet-droplet configuration and especially, the lowest frequency mode, is important.

\subsection{Summary of this study}

The goals of this study are to improve the understanding of the PFMS process. In particular, improving the quality of the ribbon is relevant for the commercialization of the process. Identifying the appropriate time and length scales for the various aspects of the process is one of the main contributions of this study. As discussed in 
section 1.3, the steady behavior of the process has been modeled using analytic and numerical methods. The relationship between processing parameters and ribbon thickness, $T$, has been established. However, very little work has been done on the transient behavior of the cast.

A large range of length scales are relevant to the PFMS process. Figure 1.3 gives a summary of the $T$ variations at each relevant length scale. Table 1.5 lists the time scales, $\tau$, over which the observed $T$ variations occur. Chapter 2 provides criteria for separating 'slow' and 'fast' time scales for the PFMS process. Even though $T$ is varying through the cast, the process can be treated as 'quasi-steady' over the slow time scales. On the longest length scales $(m)$, the variations in $T$ are due to the slowly changing conditions of the substrate. The time of the cast is given by $\tau_{\text {cast }}$, which depends upon the ratio of total mass in the crucible, $M$, to the flow rate leaving the crucible (depending upon the width of the nozzle $W$, the gap height, $G$ and the overpressure, $\Delta P)$. $T$ is also observed to vary over the time scale of the wheel revolution, $\tau_{r e v}$. Small scale thickness variations $(\mathrm{cm}-\mathrm{mm})$, are often observed as surface defects on the ribbon and occur over fast time scales. Surface defects can be very detrimental to the macroscopic properties of the ribbon, particularly since $T$ is on the order of 100 microns. At this scale, surface defects can influence the bulk properties of the ribbon. The microstructural length scales often determine the material properties of the ribbon. However, these length scales are not the focus of this study. A recent work by Byrne et al., 2007 discusses the microstructural features of the ribbon produced by our experimental caster.

Different features are observed in $T$, depending on the length of ribbon over which $T$ is averaged. The changing conditions of the wheel allow us to study the transient behavior of the PFMS process. Figure 1.4 shows how $T$ varies with time 


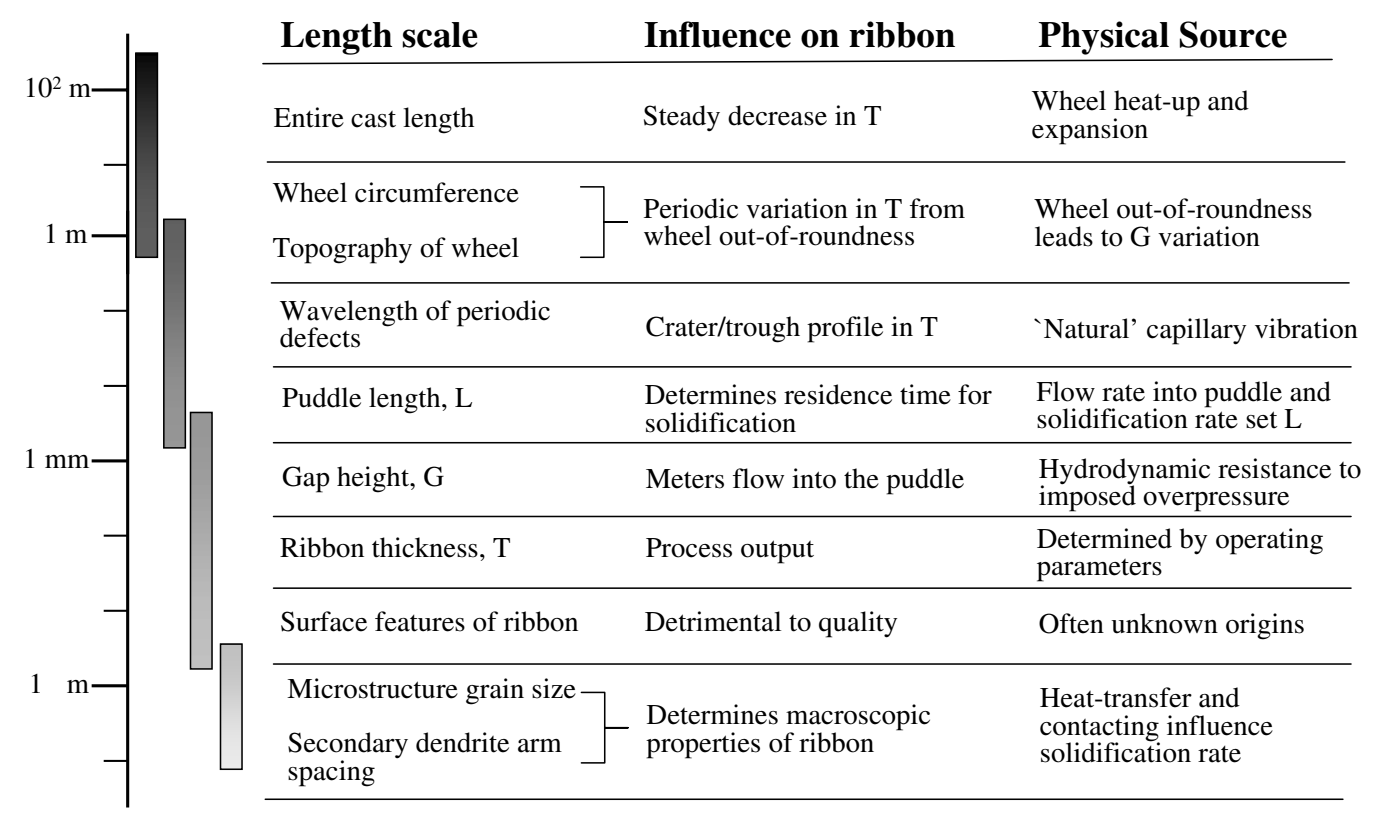

Figure 1.3: Summary of the thickness variations at each relevant length scale for the PFMS process.

Table 1.5: Summary of the time scales relevant to the PFMS process

\begin{tabular}{ccc} 
Definition & Time scale $(\mathrm{sec})$ & Description \\
\hline$\tau_{\text {cast }}=M /\left(W G\left(2 \Delta P \rho_{l}\right)^{1 / 2}\right)$ & $10^{0}$ & time of the cast \\
$\tau_{\text {rev }}=C_{w} / U$ & $10^{-1}$ & time per wheel revolution \\
$\tau_{L_{R}}=L_{R} / U$ & $10^{-2}-10^{-3}$ & time scale which $T$ is averaged \\
$\tau_{L}=L / U$ & $10^{-3}$ & puddle residence time \\
$\tau_{\text {cap }}=\left(\rho_{l} G^{3} / \sigma\right)^{1 / 2}$ & $10^{-3}$ & capillary time scale \\
$\tau_{\lambda}=\lambda / U$ & $10^{-3}$ & time scale for cross-wave defect \\
$\tau_{\text {herr }}=\lambda_{\text {herr }} / U$ & $10^{-4}$ & time scale for herringbone defect \\
\hline
\end{tabular}


during a cast (from cast ODSU06-22). The wheel speed, $U$, for this cast was 9.6 $m / s$, thus 1 second in time corresponds to $\approx 10 m$ in cast length. This particular cast was measured by cutting the cast ribbon into $1.1 \mathrm{~cm}$ sections and measuring the width and mass of each piece (and using the known density) to determine $T$. With the exception of this cast, however, the ribbon is cut into $15 \mathrm{~cm}$ sections. The time scale which the $T$ is averaged over is given by $\tau_{L_{R}}=L_{R} / U$, where $L_{R}$ is the length into which the sections of the cast ribbon is cut. There are two trends that are clear from Figure 1.4. First, there is a steady decrease in $T$ over the 4 seconds of the cast. There is also a periodic variation in $T$, which is directly related to the out-of-roundness of the wheel. The period for this $T$ variation corresponds to the time of one revolution of the wheel. The wavelength of this variation is given by the $1.9 \mathrm{~m}$ length of the wheel circumference. A moving average of 1 circumference is also plotted in Figure 1.4 as is the 'cast-averaged' value for $T(0.122 \mathrm{~mm})$, given by averaging over $\tau_{\text {cast }}$.

The overall decrease in $T$ is attributed to the wheel not being actively cooled during the cast. The wheel temperature, $\Theta$, increases as the ribbon solidifies and heat is transferred to the wheel. The wheel thermally expands and causes $G$ to decrease by an amount $\Delta G$ as it heats up. $\Delta G$ is caused by the expansion of the wheel in the circumferential direction where $\Delta G=\Delta C_{w} /(2 \pi)$ and $\Delta C_{w}=$ $C_{w} \alpha \Delta \Theta$. Here $\alpha$ is the coefficient of thermal expansion for copper and $\Delta \Theta$ is the temperature rise of the wheel. Two wheels of different circumference are currently available for casting, with $C_{w}=1.91 \mathrm{~m}$ or $2.83 \mathrm{~m}$. $\Theta$ typically varies from $30^{\circ} \mathrm{C}$ to $100^{\circ} \mathrm{C}$ during a cast. The wheel expansion, predicted from $\Delta \Theta=70^{\circ} \mathrm{C}$, agrees well with the experimentally observed expansion. Previous studies have used a run-out meter placed on the wheel surface and recorded the read-out with a video 




Figure 1.4: Thickness, $T$, variations occur over the length of the cast. There is a steady decrease in $T$ as well as a periodic variation. The $T$ is measured by cutting the cast ribbon into $1.1 \mathrm{~cm}$ sections. A moving average of 1 circumference and the 'cast-averaged' value are also plotted. (Data from cast ODSU06-22)

camera (30 frames per second) to measure the wheel expansion (Kahn, 2000). More recently, a non-contact capacitance gauge has been incorporated into the data acquisition process during the cast. (Further details on the data acquisition system are given in Section 5.4.) Figure 1.5 shows how $G$ changes in time (from cast ODSU06-22). An overall decrease in $G$ is observed, as well as a periodic variation in time.

The casting wheel is slightly out-of-round, due to machining imperfections and the plastic deformation which has occurred from multiple castings (over 400 casts). While this imperfection is small compared to $R_{w}$, it is significant when compared to $G$. (Section 5.3.1 give detailed information about the profiles of each of our casting wheels.) The out-of-roundness causes $G$ to vary periodically as the wheel rotates. The amplitude of the periodic variation, $\delta$, is shown in Figure 1.5, along with a moving average of 1 wheel circumference and the cast averaged value for $G$ 


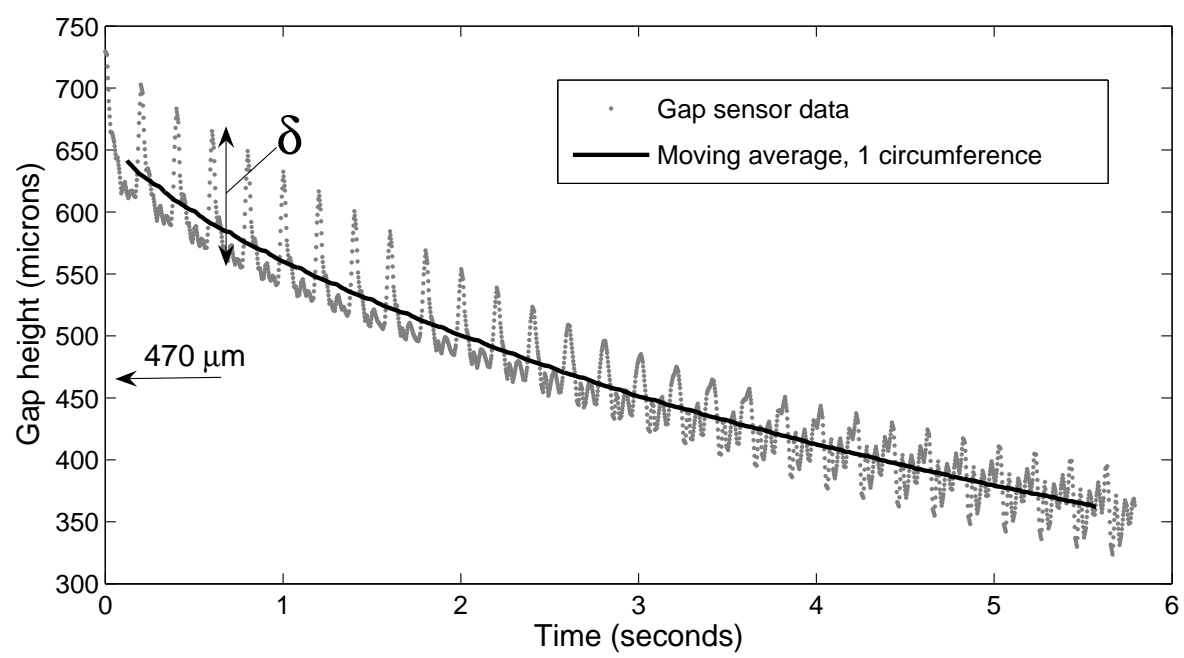

Figure 1.5: Gap height as a function of time showing i) an overall decrease ii) a periodic variation and iii) a deformation for the cold wheel profile. (Data from cast ODSU06-22)

$(0.47 \mathrm{~mm})$. The periodic behavior of $G$ is directly in phase with that of $T$. That is, when $G$ is at its highest value (per revolution), $T$ also has a local maximum. Not only does $G$ decrease throughout the cast, but the cold wheel deforms as the wheel increases in temperature, changing the profile. It is not clear how this deformation occurs (although a further discussion of this phenomena is given in Section 5.3.2).

$T$ can be predicted from knowledge of the fluid dynamics alone. $G$ tends to meter the flow from the nozzle and the time dependent variation of $G$ correlates to the behavior of $T$. However, information on the solidification rate of the ribbon is not obtained without knowledge of the $L$. The puddle is measured through the cast using a high speed video camera. Typically, an EktaPro camera at 1000 frames per second (fps) is used to image the puddle. However, a Phantom camera recording at 15,000 fps was used to capture the data shown in Figure 1.6. $L$ is then measured from analyzing the individual frames from the video. The resolution of 




Figure 1.6: Puddle length, $L$, variations occur over the length of the cast. There is an initial decrease in $L$, then a steady increase through the cast. There is also a periodic variation. $L$ is measured from high speed video analysis and the data is averaged of over the same time as in Figure 1.4. A moving average of 1 circumference is also plotted, as is the cast average value. (Data from cast ODSU06-22)

the measured $L$ is at a higher sampling rate than that of $G$ or $T$. $L$ is therefore pre-averaged over the same time scale $\left(\tau_{L_{R}}\right)$ as the $T$ data in Figure $1.4(\approx 20$ frames averaged for each $L$ data point in Figure 1.6).

The $L$ data in Figure 1.6 show some interesting dynamics. Initially $L$ decreases in time, with a periodic variation superimposed on this downward trend. The periodic variation is in phase with $T$ and $G$, where the largest value of $L$ corresponds to the largest values of $T$ and $G$ (per revolution). However, $L$ begins to turn and increase after $\approx 1$ second. The periodic variation is still observed, but $L$ steadily increases for the rest of the cast. (This behavior is documented for other casts in Section 6.4.) The $L$ measurement near the end of the cast shows more scatter 
in the data. This is due to the difficulty in determining the detachment point at which $L$ ends. The data is more noisy near the end of the cast because the ribbon quality deteriorates and the $L$ measurement is difficult to resolve.

The turning behavior in $L$ is not fully understood. The steady state mass balance gives $L V=T U$. Since $T$ is decreasing throughout the cast while $L$ is increasing, $V$ must be decreasing faster than $T$ during the cast. $L$ appears to initially follow the trend predicted from the fluid dynamics, i.e. the decrease in $G$ causes the flow rate to decrease and $L$ gets smaller. However, $L$ depends upon both the fluid flow and heat transfer of the process. A decrease in $V$ would result in $L$ increasing to keep the steady state satisfied. Both the wheel temperature, $\Theta$, and the interface conditions of the wheel are changing as the cast progresses.

Chapter 2 addresses the dynamics of casting over the slow time scales $\left(\tau_{\text {cast }}\right.$ and $\left.\tau_{\text {rev }}\right)$. The steady state mass balances coupled with a model for the thermal expansion of the wheel are used to predict $T, \Theta$ and $G$ during the cast. The variation of $V$ during the cast is modeled empirically and used to predict $L$. Research into how the thermal conditions of the wheel influence the heat transfer are ongoing.

$T$ variations also occur on smaller scales, from $\mathrm{cm}$ to $\mathrm{mm}$. The complicated dynamics of the PFMS process can often lead to defect formation in the ribbon. Chapter 3 addresses a common defect observed in our casting, referred to as the 'cross-wave' defect (or cross-stream wave defect). Figure 1.7 shows an image of the cross-wave defect. The primary physics of the of the defect are the interaction between inertial and capillary forces. The high casting speeds and low molten metal viscosity correspond to relatively high Reynolds number flows. The small Bond number indicates that gravity has little effect on the puddle and the surface tension tends to determine the puddle shape. 


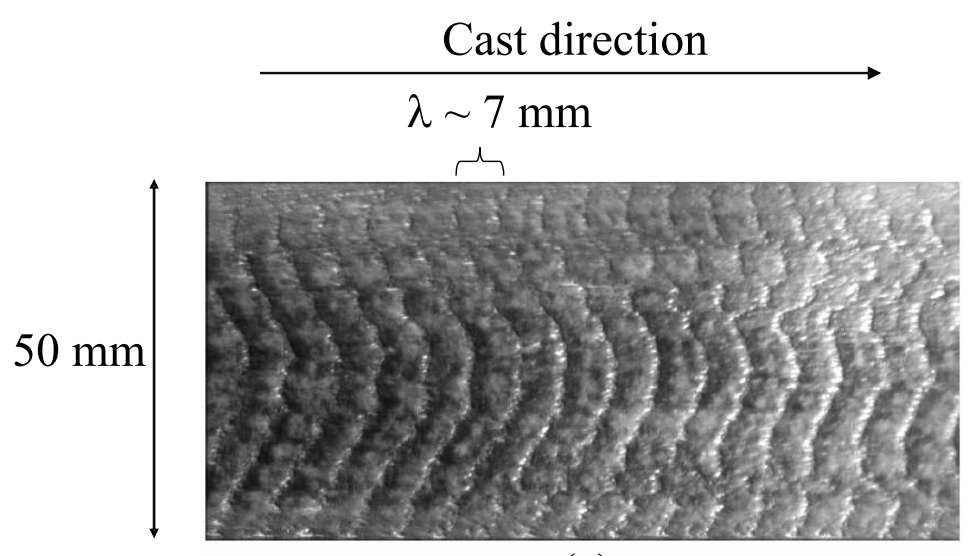

(a)

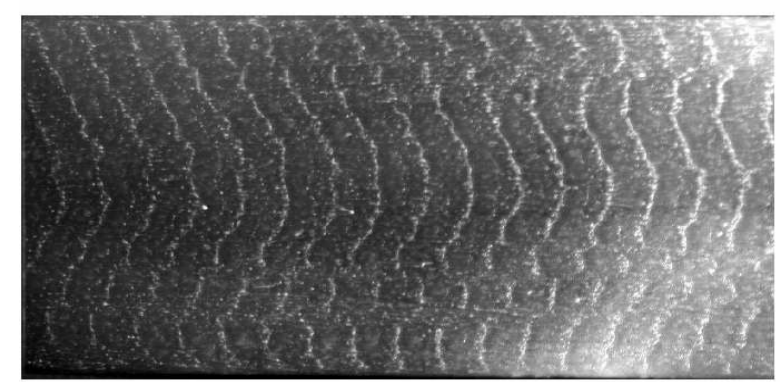

(b)

Figure 1.7: Photograph of cross-stream wave as seen on $(a)$ free side and $(b)$ substrate side of ribbon product. The wavelength $\lambda \sim 7 \mathrm{~mm}$ is seen on a ribbon section, $50 \mathrm{~mm} \times 130 \mathrm{~mm}$. The cast direction is left to right. 
We find that the molten metal puddle has 'natural' vibrations due to surface tension and these vibrations correlate with the cross-wave defect in the foil. The defect wavelength, $\lambda$, is easily measured from the ribbon. The defect formation mechanism is difficult to model before the primary physics of the puddle motions have been identified. The frequency of the cross-wave defect is measured directly as $f_{\lambda}=U / \lambda$. A dimensional analysis reveals that $f_{\lambda}$ is proportional to the inverse of the capillary time scale $\left(\tau_{c a p}=\left(\rho_{l} G^{3} / \sigma\right)^{1 / 2}\right)$, given as

$$
f_{\lambda}=C\left(\frac{\sigma}{\rho_{l} G^{3}}\right)^{1 / 2}
$$

Here the proportionality constant is found to be $C=1.94$. Motions are detected on both menisci, but the interaction between the upstream meniscus (USM) and downstream meniscus (DSM) is not obvious.

The motions of the USM are similar to the oblate to prolate oscillations of a 'plucked' sphere. The geometry of a droplet is significantly different from the PFMS puddle, yet the underlying physics of inertial and capillary force balance are the same. Constraining the droplet at solid boundaries restricts the motions and brings the geometry closer to that of the PFMS puddle. Figure 1.8 illustrates a family of model problems considered in order to link the vibrational modes of a spherical droplet to the motions of the puddle. The molten metal puddle is modeled as a $2 D$ fluid droplet confined between two plates (the nozzle and the substrate). Clearly this is an oversimplification; however, it does begin to approximate the vibrations of the puddle.

A model problem of a $2 D$ section of fluid confined by two plates is given in Section 3.7. The problem is depicted by the left schematic in Figure $1.8 \mathrm{~b}$ ). The vibrational frequencies of the meniscus are calculated. It is found that the vibra- 
a) Model 1: Vibrating Sphere (Rayleigh Modes),

$$
\omega^{2} \approx \mathrm{n}(\mathrm{n}-1)(\mathrm{n}+2)\left(\frac{\sigma}{\rho r^{3}}\right)
$$

b) Model 2: Confined 2D Fluid


c) Model 3: PFMS Puddle

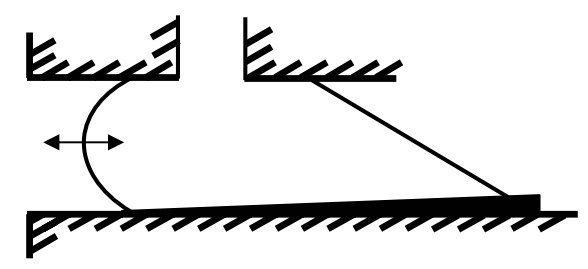

Figure 1.8: Model problem overview where in a) the classical droplet vibrations are considered while b) brings in solid boundaries to restrict the motion of the droplet. The family of models give different results when considering either fully deformable menisci or spherical cap shapes. c) The vibration frequencies predicted compare favorably with the cross-wave defect. 
tion frequencies become independent of the channel depth for large aspect ratios (comparable to the puddle geometry). The predicted vibration frequency for the mode $m=2$ (i.e. the mode that most closely resembles the USM motion) is given by $f=2.55 / \tau_{\text {cap }}$. The constant of 2.55 should be compared to the experimental value of $C=1.94$.

An alternative model problem is given in Appendix A. The vibrations of a $2 D$ section of fluid confined between two parallel plates are considered, where the menisci shapes are restricted to circular cap shapes. The problem is depicted by the right schematic in Figure $1.8 \mathrm{~b}$ ). Disturbances to the menisci must conserve mass, shifting the center-of-mass of the system. The frequency of the translational vibration of the center-of-mass of the system scales inversely with the aspect ratio of the fluid. Therefore, this type of meniscus interaction does not appear to correspond to the scaling of the vibrations of the PFMS puddle.

Considering a family of model problems to identify the physics of the cross-wave defect has led a more generalized problem of the vibrations of coupled capillary surfaces. The vibration frequency of a spherical droplet of fluid surrounded by a passive gas are a classical example of a capillary/inertia balance. As summarized by Lamb, 1932, Lord Rayleigh, 1879, predicted the frequency of small oscillations of a fluid droplet (mode $n$ ) as,

$$
\omega_{n}=\left[n(n-1)(n+2)\left(\sigma / \rho r^{3}\right)\right]^{1 / 2}
$$

where $\sigma, \rho$ and $r$ are surface tension, liquid density and radius of the undeformed sphere. Note that Equation (1.3) has a similar scaling to Equation (1.2), where the gap height, $G$, in $\tau_{\text {cap }}$ has been replaced by $r$.

In Chapter 4, we extend the modeling work from Appendix A to account for 
an axisymmetric droplet confined by a tube, with the two sections of the droplet extending from each end. Each meniscus is considered as capillary surface where the curvature of the droplet gives the pressure jump across the meniscus, as given by the Young-Laplace equation. A Newtonian force balance is applied to the center-of-mass of the coupled droplet system. The capillary pressures are the forces acting the system and equilibrium configurations occur when these forces balance. Restricting to the quasi-steady interface shape of a spherical-cap allows for an analytic model which tracks the center-of-mass of the system. A translational vibration mode which exchanges fluid between the droplets can occur with lower frequency than the traditional Rayleigh modes. The predicted vibration frequencies compare favorably to experimental values. A similar modeling approach has recently been used to study droplet motion in microfluidic devices (Daniel, Chaudhury \& de Gennes, 2005). The material in Chapter 4 is a departure from the study of the PFMS process. However, the motivation for the study was directly inspired by the motions of the molten metal puddle.

In studying the dynamics of the PFMS process, we have chosen to work from the largest length scales, $m$, down to the intermediate ones where defects commonly occur, $\mathrm{cm}$. This is in contrast to most of the research in this field which focuses on the microstructural length scales. While our casting group does study microstructural variations, (e.g. Byrne et al., 2007), they are not the focus of this thesis. Rather, we address the issues of transient behavior through the cast and the quality of the macro-scale features of the ribbon.

Chapter 5 looks at the experimental setup of the PFMS caster. The changes to the data acquisition are documented, which include modifications of the casting 
superstructure and recent equipment for monitoring $G$ during the cast. Finally in Chapter 6, the ongoing work is summarized and future studies are suggested. 


\section{Chapter 2}

\section{Transient Behavior of the Planar-flow}

\section{Melt Spinning Process*}

\section{$2.1 \quad$ Introduction}

The focus of this chapter is on the continuous processing aspects of casting a uniform thickness product. The ribbon thickness, $T$, is the main output variable for the process and often effects the material properties of the ribbon (Das et al., 1997; Karaaslan et al., 1998; Meco \& Napolitano, 2005). Controlling $T$ is therefore of significant interest. It is important to understand how the PFMS process responds to changing conditions which can lead to $T$ variations in the cast product. We present data which show the transient behavior of the process over the entire cast length and during each revolution of the wheel. As discussed in section 1.4, the experimental casting conditions are slowly varying during the time of the cast. These changing conditions are attributed to 1) the time dependent behavior of the gap height, $G$, and 2) the wheel temperature, $\Theta$, increasing during the cast because the wheel is not actively cooled. The former is a mechanical disturbance while the latter influences the heat transfer, even though there is a coupling as clarified below. Both influence the size of the molten metal puddle and ultimately $T$.

A number of the processing parameters are varying during the cast. The wheel acts as a heat sink to remove the energy required for solidification and the wheel

${ }^{*}$ An enhanced paper based on the work in this chapter will be submitted as: E.A. Theisen, M. J. Davis, S. J. Weinstein and P. H. Steen, "Transient Behavior of the Planar-flow Melt Spinning Process". 
temperature, $\Theta$, increases as the cast progresses. There is a steady decrease in $T$ throughout the cast length even as the puddle length, $L$ is found to increase. The wheel is slightly out-of-round, causing $G$ to vary periodically in time. It is observed experimentally that $L$ and $T$ also vary periodically, in phase with $G$. The wheel also thermally expands as it is heated, causing $G$ to decrease during the cast. Therefore, the change in $\Theta$ not only influences the thermal conditions of the wheel, but also couples to the mechanical disturbance of the variations in $G$. The wheel is found to expand in a nonuniform manner and deform from its initial profile.

An overview of the experimental casting results is given in Section 2.2. A brief discussion of the caster operation and the data acquisition system are given in section 2.2.1. The transient measurements of the cast data are provided in Section 2.2.2. Section 2.3 presents a dynamical model of the process that predicts the observed experimental results. Section 2.3.1 provides a model that predicts the wheel heat-up and expansion during the cast, giving estimates for $\Theta(t)$ and $G(t)$. In Section 2.3.2, an analytic model for $T(t)$ and $L(t)$ is developed by using control volume mass balances around the solid and liquid regions of the contact zone. Section 2.3.3 uses a steady momentum balance to couple the fluid flow into the puddle with $G(t)$, which is treated as a forcing function. Then in Section 2.3.4, an empirical model is provided to account for the decreasing solidification velocity, $V(t)$, that is observed to occur as the cast progresses. A summary of the results are provided in Section 2.4 and a further discussion of the dynamical model is given in Section 2.5. 


\subsection{Experimental results}

\subsubsection{Caster operation and data acquisition}

The PFMS process feeds molten metal from a crucible (due to imposed overpressure $\Delta P)$ through a nozzle of size $B$ and into a narrow gap region between the nozzle and casting wheel (or substrate), see Figure 1.1. The $\Delta P$ consists of a metallostatic head and an applied gas pressure. An inert gas pressurizes the crucible and is ramped through the cast to compensate for the decreasing head of metal, keeping $\Delta P$ constant. Because of the small gap, $G$, the nozzle interferes with the flow, and a puddle of molten metal held by surface tension is formed. The contact between the wheel and molten puddle is such that enough heat is removed from the melt for solidification to occur. The solidification front moves at an average velocity, $V$. The wheel (moving with linear velocity $U$ ) continually removes the solidified product from the contact zone. $U$ is maintained constant during a cast. The final ribbon thickness, $T$, is controlled by the parameters $B, G, \Delta P, V$, and $U$ (as discussed in Section 1.3.1). Aluminum and Al-7\% Si alloys are the typical materials cast.

Our experimental casting wheel is a copper-0.4\%beryllium $(\mathrm{CuBe})$ rim which is $1.9 \mathrm{~m}$ in circumference, $13.5 \mathrm{~cm}$ wide and $1.27 \mathrm{~cm}$ deep. The cylindrical $\mathrm{Cu}-\mathrm{Be}$ rim is fixed between two aluminum side plates which are mounted on a rotating axel. The wheel is slightly out-of-round, which causes $G$ to vary periodically as the wheel rotates. The out-of-roundness is caused by small imperfections in the machining of the substrate as well as plastic deformation from the wear over time. Even though the out-of-roundness is small compared to the wheel diameter, it can be large when compared to $G$. The depth of the wheel $\left(W_{D}=1.27 \mathrm{~cm}\right)$ and 
the thermal diffusivity of the wheel $\left(\kappa=\rho_{w} /\left(k_{w} C_{p, w}\right)=1.16 \mathrm{~cm}^{2} / s\right)$ provide a characteristic thermal diffusion time $\left(\tau_{\text {Diff }}=W_{D}^{2} / \kappa \approx 1.4 s e c\right)$. The wheel acts as a semi-infinite heat sink when compared to the residence time of the puddle $\left(\tau_{L}=U / L \approx 10^{-3} \sec \right)$, i.e. $\tau_{\text {Diff }} \gg \tau_{L}$. The heat that is transferred to the wheel while under the puddle diffuses into the $\mathrm{CuBe}$ during the following wheel revolution. The wheel temperature, $\Theta$, steadily increases during the cast. Prior to each cast, the wheel is polished with 150 grit sand paper to maintain a clean surface of uniform roughness. However, the casting is done in an ambient environment and no cleaning of the wheel surface is done during the cast. Table 1.4 lists the geometry and physical properties of the wheel.

The time dependent measurements of the processing variables $U, P_{a p p}, \Theta$, and $G$ are recorded with a data acquisition system (National Instruments card PCI-MIO16E-4 and Labview software). $U$ is monitored with an rpm sensor placed on the axel of the wheel and is held constant during a cast. A pressure transducer reads the applied pressure, $P_{a p p}$, to the crucible. The overpressure, $\Delta P$, consists of $P_{a p p}$ and the pressure from the metallo-static head. $P_{a p p}$ is ramped with a Proportion $\mathrm{Air}^{T M}$ solenoid value to maintain $\Delta P$ constant as the head of metal decreases during the cast. $\Theta$ is measured using a fast-response K-type MedTherm thermocouple imbedded $2 \mathrm{~mm}$ below the wheel surface in the center of the wheel. A non-contact capacitance gauge is used to measure $G$ during the cast (MTI Instruments model AS-900 with ASP-20-CTA probe). Further information on the experimental setup are given in detail elsewhere (Carpenter \& Steen, 1997; Reed, 2001).

Typically, $\approx 1 \mathrm{~kg}$ of molten metal is cast which results in nearly $50 \mathrm{~m}$ of ribbon in $\approx 5$ seconds of cast time (at a nominal $U=10 \mathrm{~m} / \mathrm{s}$ ). The entire length of the cast ribbon is then measured by cutting the cast into sections of length $L_{R}$ 
(typically $15 \mathrm{~cm}$ ). Measuring the mass, length, and width of each section (along with the density) gives an average value for $T$ over the length $L_{R}$ (or alternatively over the time scale $\left.\tau_{L_{R}}=L_{R} / U\right)$.

A high speed video camera is used to measure $L$ through the cast (either an EktaPro camera recording at up to 6000 fps or Phantom camera recording at 15000 fps). A back-lighting technique is used to obtain profiles of the puddle menisci, similar to the method reported by Wilde \& Matthys, 1992; Ibaraki, 1996. Figure 2.1 shows two composite images of the puddle as captured by the high speed video camera. The images show how $L$ changes within one revolution of the wheel. A schematic of the nozzle is placed above each image to indicate the position of nozzle slot. The images are analyzed, using National Instruments Visual Assistant software, to determine the $L$ through the cast. The double-arrow line indicates the measurement $L$ from the two images is given in Figure 2.1. The $L$ measurement has a higher uncertainty than the other data, due in part to the difficulty is visualizing the tri-junction point where the ribbon leaves the puddle.

\subsubsection{Experimental data}

Figure 2.2 shows the experimental results for $T, G, \Theta$ and $L$ through a typical cast (data from cast ODSU06-22). The other operating conditions $(U=9.5 \mathrm{~m} / \mathrm{s}$ and $\Delta P=5000 P a)$ are held constant during the cast. Measurements of $G, \Theta$ and $L$ are taken at a sampling rate higher than the time which $T$ is averaged over $\left(\tau_{L_{R}}=15 \mathrm{~cm} / U \approx 0.016\right.$ seconds $)$. Each data point in Figure 2.2 is therefore averaged over the same $\tau_{L_{R}}$ scale. (Section 1.4 gives the results over a shorter time scale, corresponding to $1.1 \mathrm{~cm}$ of cast ribbon, giving a greater resolution to the 


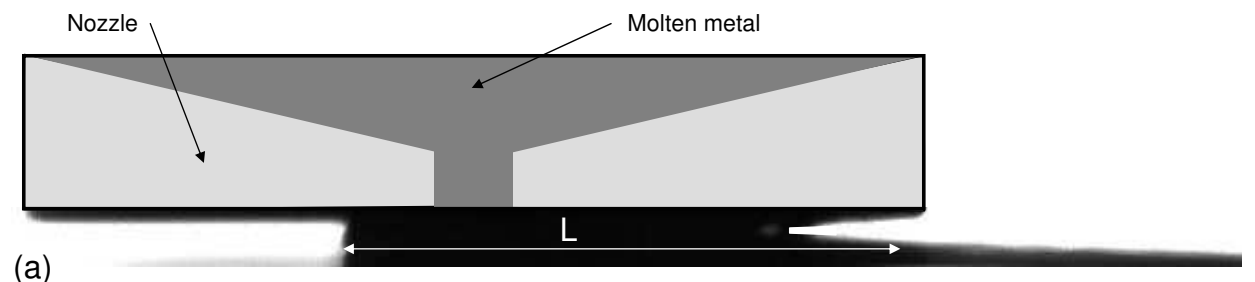

(a)

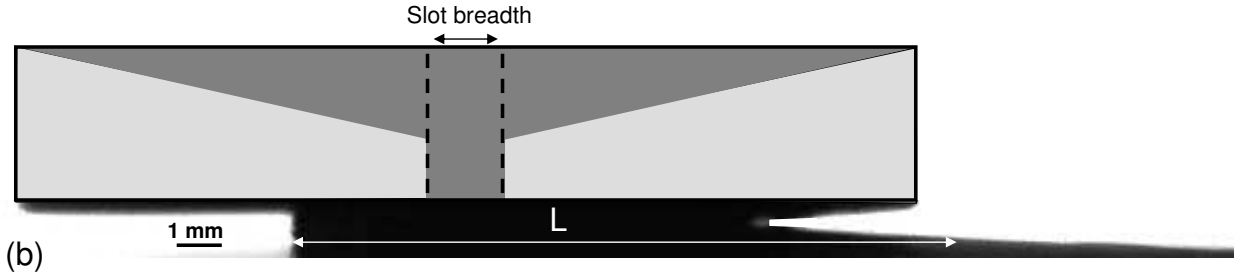

Figure 2.1: A schematic of the nozzle is superimposed on high speed video images of the puddle. The horizontal length scale of the schematic matches the image and the slot breadth of the nozzle is indicated. The vertical scale of the schematic has been compressed for clarity. The images show how the puddle length, $L$, varies during a single revolution of the wheel where (a) shows the smallest length and (b) shows the largest. An arrow is superimposed on the images to indicate the measured value of $L$. (Images are from cast ODSU06-22.) 

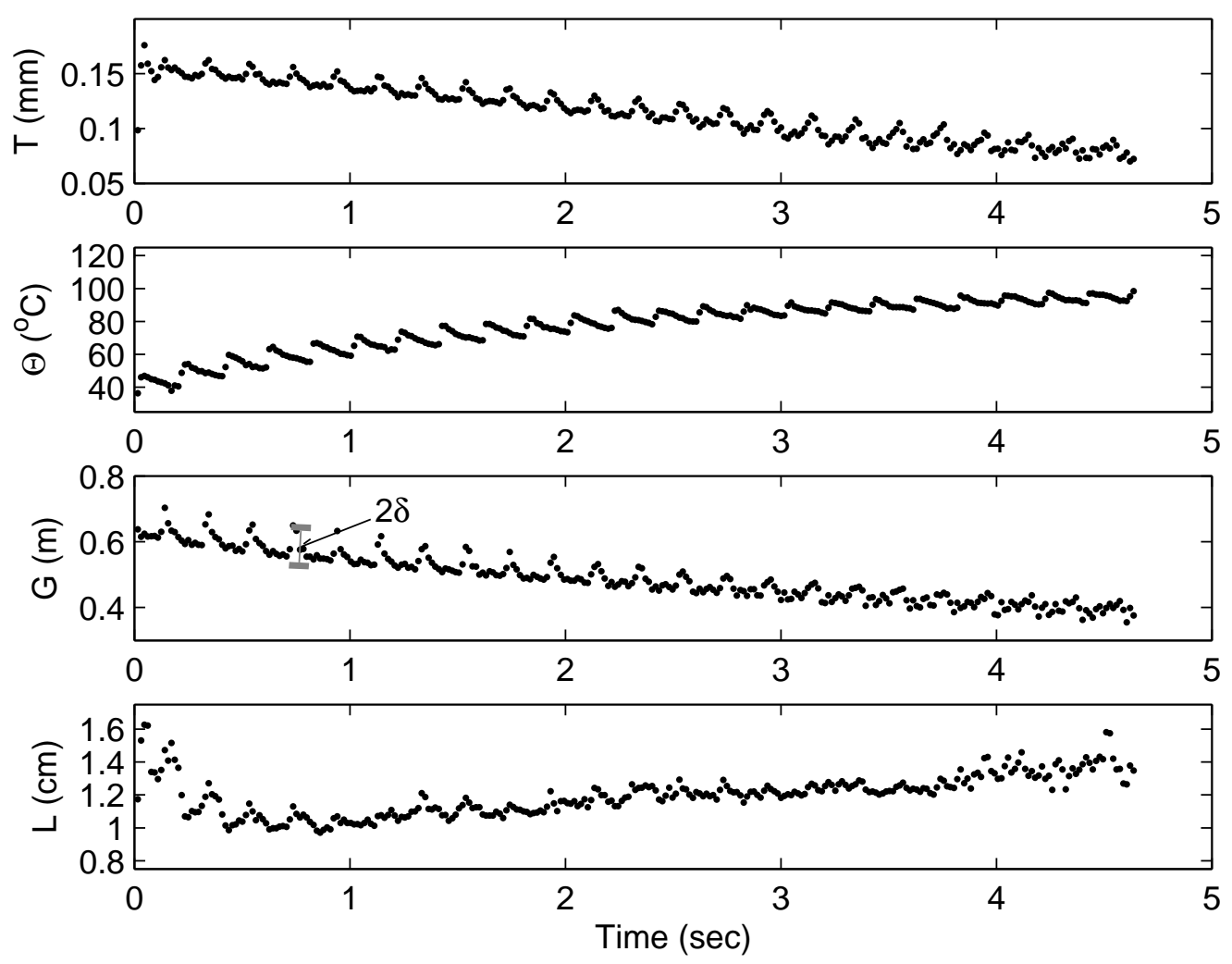

Figure 2.2: Experimental data of ribbon thickness, $T$, wheel temperature, $\Theta$, gap height, $G$, and puddle length, $L$, as a function of time during the cast. The amplitude of the wheel-out-of-roundness, $\delta$, is shown on the measured value of $G$. (Data from cast ODSU06-22)

data. However, the same general trends in the experimental data are observed for the average over the $15 \mathrm{~cm}$ length scale.)

Two trends are clear when looking at the variation of $T$ with time. First there is an overall decrease with time and second there is a periodic variation. The same decreasing and periodic trends are observed in $G$ through the cast. The periodic variation in $G$ is due to the wheel out-of-roundness. The amplitude of the out-ofroundness is given by $2 \delta$, as shown in Figure 2.2. Note, however, that $G$ not only 
decreases throughout the cast, but out-of-roundness of the wheel also decreases with time.

The wheel is not actively cooled during the cast, causing $\Theta$ to increase from 30 to $100{ }^{\circ} \mathrm{C}$ over the time of the cast, as shown in Figure 2.2. The increase in $\Theta$ causes the wheel to thermally expand and is the source of the overall decrease in $G$ through the cast (as discussed further in Section 2.3.1). $\Theta$ appears periodic, similar to the $T$ and $G$ data. However, the periodicity of $\Theta$ occurs because the thermocouple is fixed in the frame of the wheel. The measurement of $\Theta$ spikes when the thermocouple passes under the molten metal puddle and drops during the subsequent revolution of the wheel. Overall, $\Theta$ steadily increases through the cast causing the thermal conditions of the wheel to constantly vary.

The most surprising of experimental data is the $L$ measurement. Initially $L$ decreases, but then turns and steadily increases for the rest of the cast. $L$ also varies periodically, similar to $T$ and $G$. However, the data is more scattered than $T$ or $G$ and Figure 2.3 is included to show the frequency content of the puddle motions. A clear peak in the fast fourier transform (FFT) of the $L$ data is observed at $5 \mathrm{~Hz}$ and corresponds to the frequency of the wheel rotation. Higher harmonics of the primary frequency are observed in the FFT at 10, 15 and $20 \mathrm{~Hz}$. It should be noted that the measurements of $T, G$ and $L$ are all in phase with each other, with the maximum value for each occurring at the same time (for each wheel revolution).

\subsection{Modeling of the process dynamics}

An analytic model for the dynamics observed during the casting is sought. A brief overview of the modeling approach is given. Control volume mass balances 


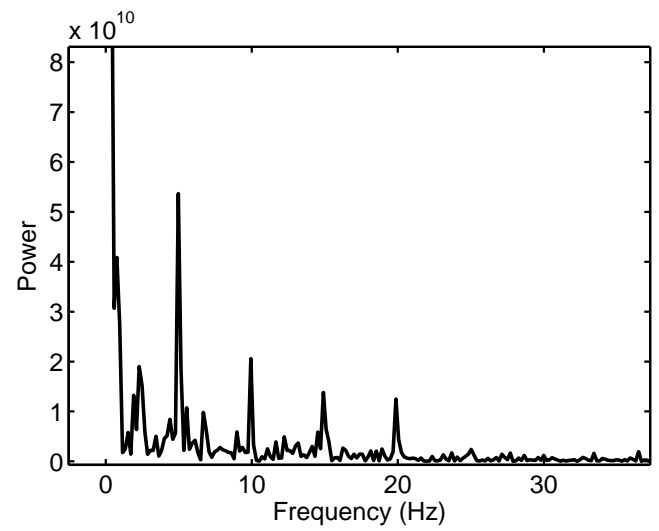

Figure 2.3: Fast fourier transform of puddle length, L, data showing dominant frequency at $5 \mathrm{~Hz}$, corresponding to the frequency of the wheel rotation. Resonant peaks are observed at 10, 15 and $20 \mathrm{~Hz}$. The out-of-roundness of the wheel causes the gap height to vary as the wheel rotates and ultimately correlates with $L$.

around the contact zone and the solidifying ribbon are developed to equate $T(t)$ and $L(t)$ to the changing conditions of the process. The contact zone is divided into two control volumes (or areas per unit width), $A_{1}$ and $A_{2}$, as shown in Figure 2.4 a). Here $A_{1}$ represents both the liquid puddle and the solid ribbon while $A_{2}$ is the solidified ribbon. Figure $2.4 \mathrm{~b})$ shows the geometric parameters $(G, D, L$ and $T$ ) which define $A_{1}$ and $A_{2}$. The mass balances result in a dynamical system that reduces to a quasi-steady balance relating $T(t) U$ to the ratio of flux of material into the puddle, $B u_{i n} . L(t)$ reduces to the ratio of $B u_{i n}$ to the solidification velocity, $V$. A steady momentum balance is then used to relate the processing parameters $\Delta P$ and $G$ to $u_{i n}$. The experimental measurements of $T, L$ and $U$ through the cast allow for $V$ to be inferred directly during the cast. The following assumptions are made to allow us to understand the basic underlying physics of the puddle:

1) The solidification front is linear and the mushy zone is negligible. The solidified ribbon is then triangular in shape as shown schematically in Figure 2.4. 


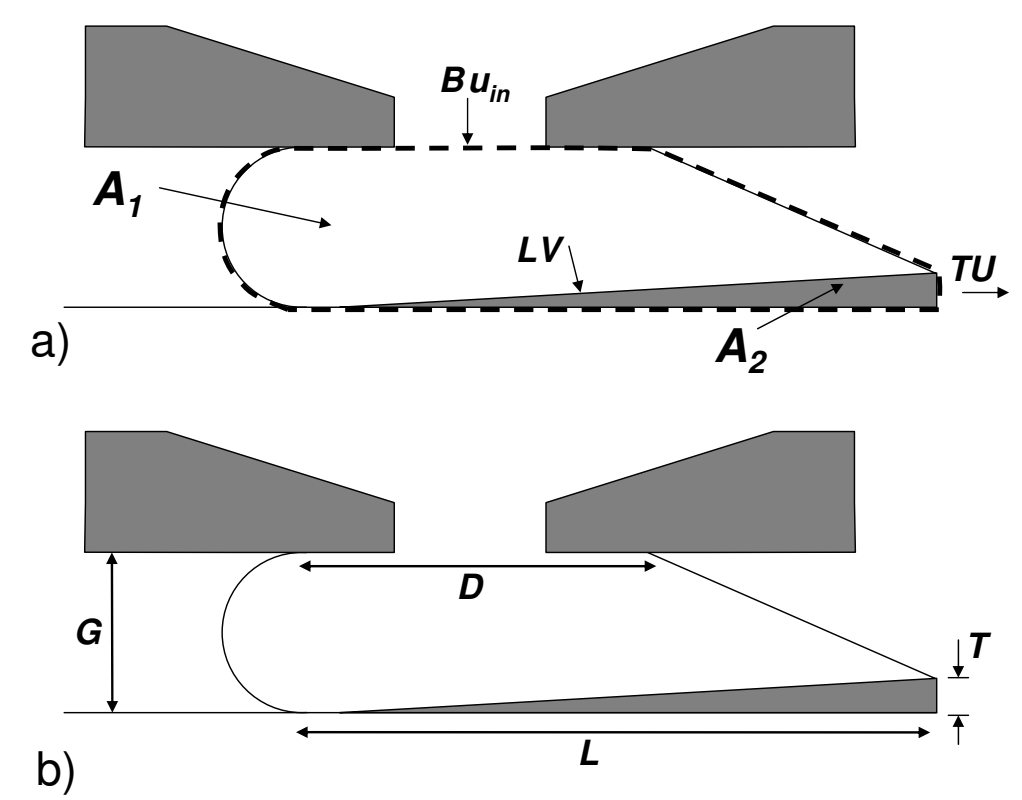

Figure 2.4: a) Control volumes $A_{1}$ (the puddle and solid regions) and $A_{2}$ (the solid region) used for the dynamical system. b) The control volumes are defined by the geometric variables $G, L, D$ and $T$.

2) The puddle can change in size in a quasi-steady manner where the upstream meniscus remains semicircular in shape and the downstream meniscus remains a linear interface.

3) The density change upon solidification is neglected since $\rho_{s} / \rho_{l} \approx 1.1$.

4) The length of the solid/liquid interface, $S$, is approximated by $L$. For typical parameters, $T / L \sim O\left(10^{-2}\right)$ and $S=L\left[1+(T / L)^{2}\right]^{1 / 2}=L\left(1+10^{-2}\right) \sim L$.

5) The solidified ribbon leaves the substrate at the melting temperature of the material. This is the highest temperature that the ribbon could be and thus corresponds to a lower bound on the heat that must be transferred to the wheel.

6) The heat diffuses rapidly through the wheel after the wheel leaves the contact zone. That is, suppose one were to move with a fixed position on the wheel. There is a thermal pulse when passing under the contact zone. It is assumed that this 
pulse is dissipated through the mass of the wheel to give an overall increased, but uniform temperature, by the time the substrate again passes under the contact zone.

The analytical model that is developed to predict the behavior of $T$ and $L$ during the cast consists of two separate calculation steps. The first involves predicting the temperature rise of the wheel, $\Delta \Theta$, depending on the amount of ribbon solidified. The thermal expansion of the wheel is calculated to give the overall decrease of the gap height, $\Delta G$, during the cast. Section 2.3.1 provides a model that predicts $\Theta(t)$ and $G(t)$. The second calculation step involves an analytic model for $T(t)$ and $L(t)$, as discussed in Section 2.3.2. This is developed by combining control volume mass balances with a steady momentum balance, given in Section 2.3.3. $T(t)$ is predicted by using the calculated value of $G$ in a quasi-steady manner. Calculating $L(t)$ is slightly more complicated, as it depends on both the fluid flow and the heat transfer of the process. In Section 2.3.4, the experimental data showing a decrease in $V$ is presented and an empirical model for $V(t)$ is determined. This model is finally applied to other cast data to predict $L(t)$.

Section 2.4 gives the results of the dynamical model. Iterating between the two calculation steps provides for time dependent predictions of $T, L, \Theta$ and $G$ based on the processing parameters. The predicted results are compared to the experimental values. Section 2.5 provides a further discussion of the results and identifies the time scale over which the quasi-steady balances are valid.

\subsubsection{Wheel heat-up and expansion}

Tables 1.1, 1.2 and 1.4 list the parameters needed to calculate the wheel heatup and expansion. In order to calculate the heat transferred to the wheel, the 
temperature at which the ribbon leaves the wheel needs to be estimated. The ribbon must be at the melting point for solidification to occur. Therefore the enthalpy change of solidification and the sensible heat of the molten metal must be transferred to the the wheel. This will be a lower bound for the calculation, since it is possible that the ribbon cools below the melting temperature before detaching. The heat transferred to the wheel, $Q$, can then be determined as the cast progresses by tracking the amount of ribbon solidified as

$$
Q(t)=\left(\Delta \nu+C_{p, l} T_{h}\right) M_{r}(t)
$$

Here $\Delta \nu$ is the enthalpy change of solidification, $C_{p, l}$ is the heat capacity of the molten metal, $T_{h}$ is the superheat of the molten metal and $M_{r}(t)$ is the mass of the solidified ribbon, which increases as the cast progresses. The mass and the heat capacity of the wheel can then be used to estimate the temperature increase of the wheel as

$$
\Delta \Theta(t)=\frac{Q(t)}{C_{p, w} M_{w}},
$$

where $C_{p, w}$ is the heat capacity of the wheel and $M_{w}$ is the mass of the wheel. The control volume for the heat balance to the wheel determines $M_{w}$. Figure 2.5 a) shows the cross-section of the wheel and b) indicates the width of the wheel beneath the ribbon that is used to determine the volume of the wheel. The dashed line in Figure 2.5 gives control volume of the wheel used to calculate $M_{w}(=$ $\left.\rho_{w} C_{w} W_{W} W\right)$. The coefficient of thermal expansion then gives the increase in size of the wheel. The circumference of the wheel is much greater than the rim thickness, thus the thermal expansion occurs mainly in the circumferential direction. That is, 
(a)

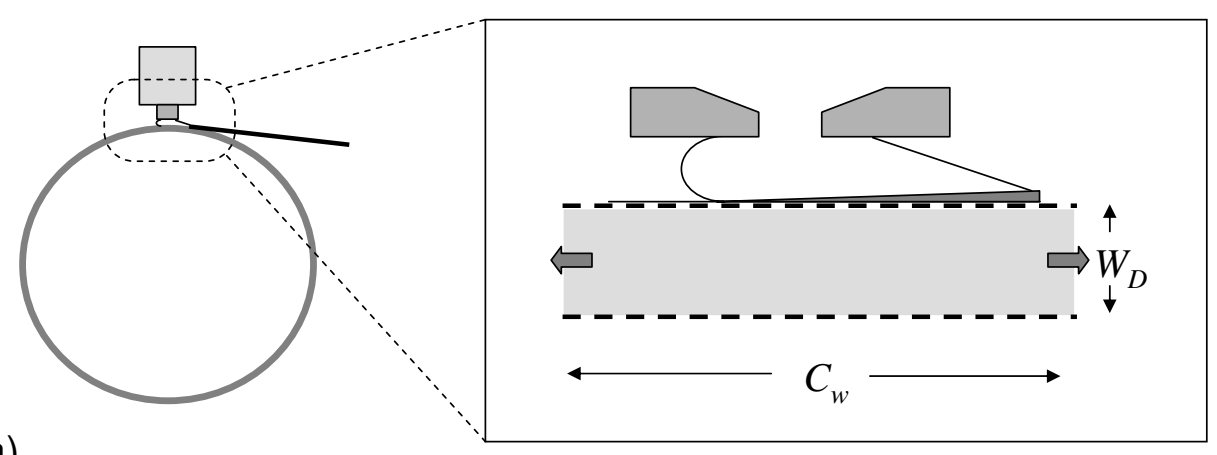

(b)

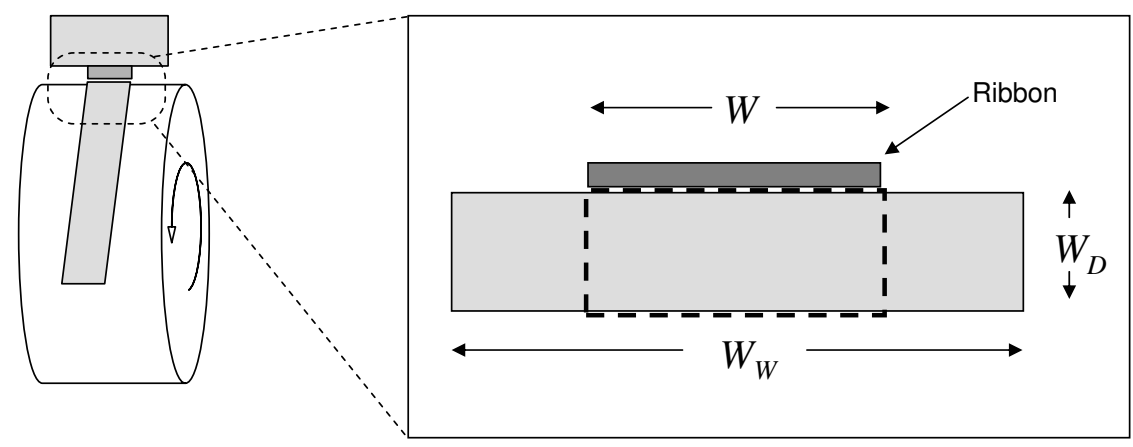

Figure 2.5: The control volume for the heat transfer that is used to calculate the temperature rise and expansion of the wheel is indicated by the dashed lines. a) shows the cross-section of the wheel and b) indicates the width of the wheel beneath the ribbon that is used to determine the volume of the wheel. 
$G$ decreases with time because the circumference of the substrate increases. The increased length of the circumference, $\Delta C$, is given by

$$
\Delta C(t)=C_{w} \alpha \Delta \Theta(t)
$$

where $C_{w}$ is the initial circumference of the wheel, and $\alpha$ is the coefficient of thermal expansion of the wheel. The gap contraction, $\Delta G$ can therefore be calculated as

$$
\Delta G(t)=\frac{\Delta C(t)}{2 \pi}
$$

Based on the amount of ribbon solidified, we get an estimate for the expansion of the substrate. This calculation gives the overall expansion of the wheel, and therefore the decrease in $G$. It does not, however, account for the periodic variation in $G$ due to the wheel out-of-roundness. It also does not account for any thermal stresses in the wheel. Rather, is assumes that the heat is uniformly distributed throughout the mass of the substrate. An approximation of $G(t)$ that accounts for the wheel out-of-roundness is given as

$$
G(t)=\underbrace{G_{i}}_{\text {measured }}+\underbrace{\delta(t)}_{\text {fit }} \sin (\omega t)-\underbrace{\Delta G(t)}_{\text {estimated }},
$$

where $G_{i}$ is the initial gap height, $\delta(t)$ is the amplitude of the wheel out-ofroundness, and $\omega$ is the frequency at which the wheel rotates. Figure 2.6 separates the time varying terms of $G$ where the a) shows the periodic variation, b) shows the overall decrease in $G$ and c) plots the sum of the two, giving a prediction of $G(t)$. A comparison between the predicted and experimental values of $G(t)$ is provided in Figure $2.6 \mathrm{c}$ ). As shown in Figure 2.2, the wheel out-of-roundness, $\delta$, varies as the cast progresses and the wheel deforms as it is heated. However, 
it is observed experimentally that the deformation can be modeled as a linearly decreasing function of time as $\delta(t)=\delta_{i}-k t$, with $\delta_{i}=50 \mu \mathrm{m}$ and $k=7.5 \mu \mathrm{m} / \mathrm{s}$. Here $\delta_{i}$ is determined from the out-of-roundness of the wheel prior to casting and $k$ is the rate at which the wheel deforms. While this is a fitting function for $\delta(t)$, the wheel expansion is very reproducible and the fit is based on experimental results. It also keeps with the control-volume approach we are using to predict the cast behavior.

\subsubsection{Dynamical model of puddle size}

A control volume approach for the mass balances is developed for analyzing the dynamics of the PFMS process. This provides a means for the puddle to change in size during the cast in a manner that is realistic yet tractable. $T(t), L(t)$ and $G(t)$ provide degrees-of-freedom by which the puddle can change in size, although $G(t)$ is ultimately prescribed by Equation (2.5). A $2 D$ model is appropriate since the nozzle width is much greater than the gap height $(W \gg G)$. The contact zone is divided into two control volumes (or areas per unit width), $A_{1}$ and $A_{2}$, as shown in Figure 2.4 a). Here $A_{1}$ represents both the liquid puddle and the solid ribbon while $A_{2}$ is the solidified ribbon. Figure $2.4 \mathrm{~b}$ ) shows the geometric parameters $(G, D, L$ and $T)$ which define $A_{1}$ and $A_{2}$. The areas can be written as $A_{1}=\pi G^{2} / 8+G D+T(L-D)+(G-T)(L-D) / 2$ and $A_{2}=T L / 2$.

The mass balances account for the flux of material through the boundaries of the control volumes. At steady state, the 'feed rate', the 'freeze rate' and the 'pull rate' are in equilibrium. Molten metal is fed through the nozzle at a rate of $B u_{i n}$, (per unit width) where $u_{i n}$ is the average flow velocity at the puddle inlet. The

freeze rate is given by $L V$, where $V$ is the average solidification velocity. The pull 
a)

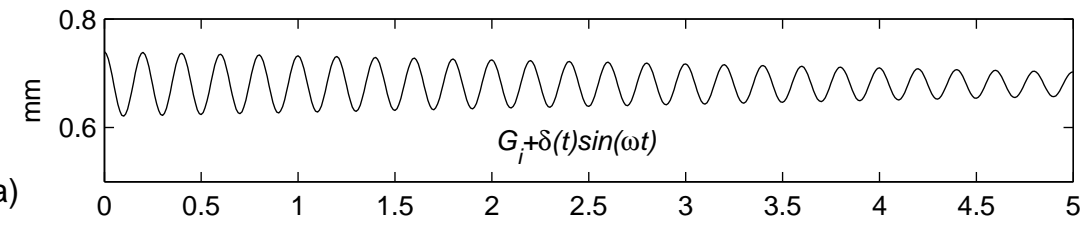

b)

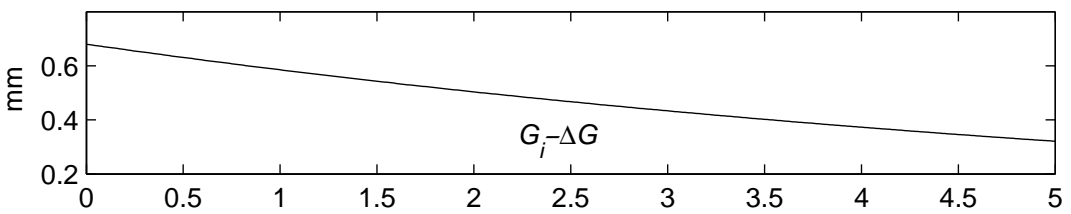

c)

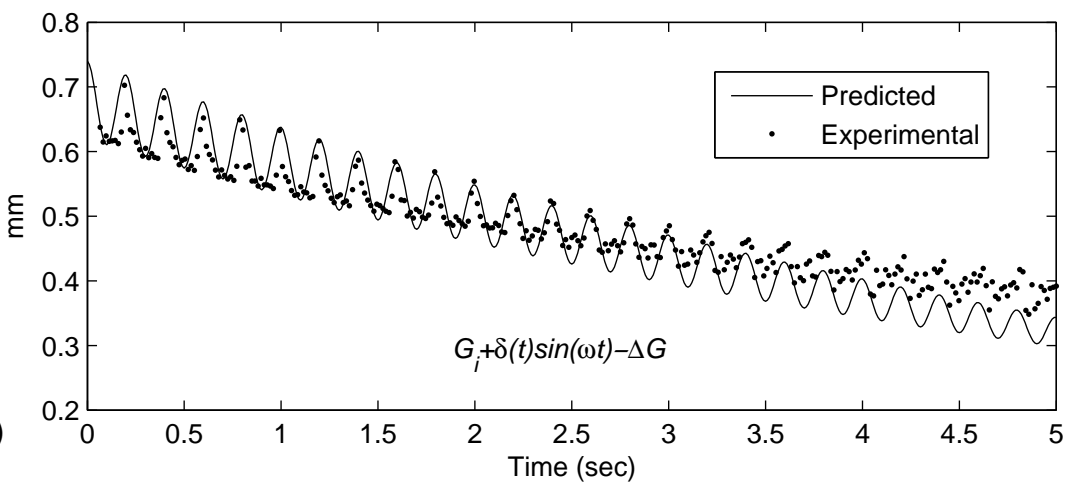

Figure 2.6: The time dependent behavior of $G$ is modeled by combining a) the periodic variation due to the wheel out-of-roundness with b) the overall wheel expansion given by $\Delta G$. c) The sum of the two effects provide an estimate for $G(t)$, which is plotted along with experimental data. A linear decrease in the wheel out-of-roundness, $\delta(t)$, has been applied to account for the deformation of the wheel through the cast. (Data from cast ODSU06-22) 
rate is given by, $T U$, which is the rate at which the metal leaves the contact zone. A control volume around each area gives a dynamical system of the form

$$
\begin{aligned}
& \frac{d A_{1}(t)}{d t}=B u_{i n}-T(t) U \\
& \frac{d A_{2}(t)}{d t}=L(t) V-T(t) U .
\end{aligned}
$$

At steady state, $A_{1}$ and $A_{2}$ must be constant, therefore, $B u_{i n}=\bar{L} V=\bar{T} U$, where $\bar{T}$ and $\bar{L}$ indicate the steady state values. Since the puddle menisci provide flexible boundaries these rates need not always balance and the puddle can change size in response to disturbances. Substituting the geometric definitions of $A_{1}$ and $A_{2}$ into Equations (2.6) and (2.7) gives the following equivalent system,

$$
\underbrace{\left(\frac{\pi G}{2}+D+L\right) \frac{d G}{d t}}_{I}+\underbrace{(L-D) \frac{d T}{d t}}_{I I}+\underbrace{(T+G) \frac{d L}{d t}}_{I I I}=\underbrace{-2 U(T-\bar{T})}_{R H S-A}
$$

and

$$
\underbrace{T \frac{d L}{d t}}_{I V}+\underbrace{L \frac{d T}{d t}}_{V}=\underbrace{2 V(L-\bar{L})-2 U(T-\bar{T})}_{R H S-B} .
$$

Here $T, L$ and $G$ are time dependent variables. The left hand sides of Equations (2.8) and (2.9) can be evaluated directly from the experimental data, e.g. $d T(t) / d t \sim \Delta T / \Delta t$ where $\Delta T$ is the change in $T$ from one section of ribbon (of length $\left.L_{R}\right)$ to the next and $\Delta t$ is the time interval between the sections, $\tau_{L_{R}}$. It turns out that each term on the left hand side $(I-V)$ is small relative to the terms on the right hand side $(R H S-A$ and $R H S-B)$. This is consistent with the results given by Kahn, 2000. Indeed, by plotting the ratio of the terms, Figure 2.7 shows that the experimental measurements of terms $I-V$ are less than $5 \%$ of the values 


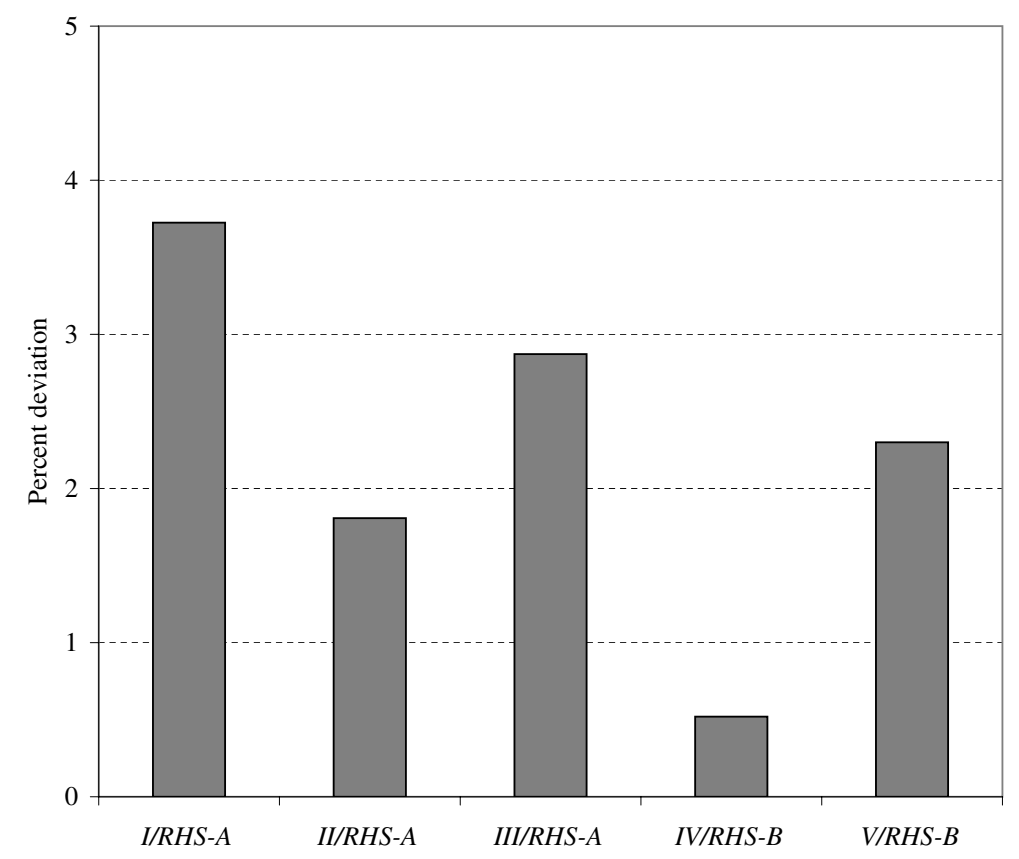

Figure 2.7: Experimental data is used to compare the magnitude each term on the left hand side of Equations (2.8) and (2.9) to that of the right hand side. The results indicate that the steady state behavior is dominant over the time scale $\tau_{L_{R}}$ over which $T$ is averaged.

of $R H S-A$ and $R H S-B$. The steady values, $\bar{T}$ and $\bar{L}$, are calculated through the cast over a moving average of one wheel circumference $\left(t=\tau_{\text {Rev }}\right)$, as discussed in Section 1.4. The results indicate that the steady state behavior is dominant on the time scale, $\tau_{L_{R}}$, over which $T$ is averaged. This indicates that for $t>\tau_{L_{R}}, T(t)$ and $L(t)$ reduce to the steady state mass balances given by

$$
\begin{aligned}
T(t) & =\bar{T}=\frac{B u_{i n}}{U} \\
L(t) & =\bar{L}=\frac{B u_{i n}}{V} .
\end{aligned}
$$

The mass balances can be used to further specify the time scales over which the Equations (2.11) and (2.10) are satisfied. The full dynamical system can be lin- 
earized by substituting $T(t)=\bar{T}+\hat{T}(t), L(t)=\bar{L}+\dot{L}(t)$ and $G(t)=\bar{G}$ (= constant) into Equations (2.8) and (2.9). Here $\dot{T}$ and $\dot{L}$ are small deviations from the steady values. Retaining the first order terms in $\dot{T}^{\prime}$ and $\dot{L}$ gives the linearized system,

$$
\begin{aligned}
& (\bar{L} \bar{G}+\bar{T} D) \frac{d \dot{L}}{d t}=-2 V(\bar{L}-D) \dot{L}-2 U D \dot{T} \\
& (\bar{L} \bar{G}+\bar{T} D) \frac{d \hat{T}^{\prime}}{d t}=2 V(\bar{T}+\bar{G}) \dot{L}-2 U \bar{G} \dot{T} .
\end{aligned}
$$

The eigenvalues for this system provide the time scale for the exponential decay to the steady state of the form $\exp \left(\gamma_{i} t\right)$ and can readily be identified as

$$
\begin{aligned}
\gamma_{i}= & \frac{1}{\bar{L} \bar{G}+\bar{T} D}[-\bar{G} U+V(\bar{L}-D)+ \\
& \left.(-1)^{i}\left[V^{2}(\bar{L}-D)^{2}-4 D V^{2} \bar{L}+(\bar{G} U)^{2}-2 V \bar{G} U(\bar{L}+D)\right]^{1 / 2}\right]
\end{aligned}
$$

where $i=1$ or 2 . Two real-valued negative $\gamma$ values are found when evaluating Equation (2.14) at the typical processing conditions listed in Table 1.1. Time-scales of $10^{-3}$ and $10^{-4}$ seconds are identified from $\gamma^{-1}$. This provides further justification for using the steady behavior predicted by Equations (2.10) and (2.11). Dynamics that occur over a 'slow' time-scale $\left(t_{s}\right)$ greater than $10^{-3}$ seconds can therefore be treated as a quasi-steady variation to the process.

The dynamic mass balances and the experimental data indicate that the steady state of $T$ and $L$ is applicable to the process when conditions are changing at a rate slower than $t_{s}$. It is implied from here on that we are considering $t>t_{s}$ variations to the process and that $G(t)$ predicted by Equation (2.5) can be equated to the quasi-steady state $\bar{G}$. The mass balances do not, however, provide information about momentum effects within the puddle. Therefore, a further balance is needed to establish the coupling between the $\bar{G}$ and $u_{i n}$. 


\subsubsection{Steady Bernoulli balance}

In order to understand how the processing parameters $\bar{G}$ and $\Delta P$ relate to $u_{i n}$, a steady momentum balance is applied to the molten metal in the crucible. It is important to note that $u_{i n}$ is not constant in our process, rather $\Delta P$ in the crucible is held constant. Therefore, as $G$ decreases the hydrodynamic resistance to flow increases, leading to a decrease of $u_{i n}$. Assuming no irreversible losses and irrotational flow (due to the small kinematic viscosity of molten metal) a Bernoulli balance gives the relation

$$
u_{i n}=\frac{\bar{G}}{B}\left(\frac{2 \Delta P}{\rho_{l}}\right)^{1 / 2},
$$

where $\Delta P$ is the overpressure in the crucible, $\rho_{l}$ is the liquid density and $B$ is the nozzle breadth. (Section 1.3.1 provides a further discussion of Equation (2.15)). Combining Equations (2.5), (2.10) and (2.15) give a predicted value for $T(t)$ which agrees well with experimental results, as shown in Figure 2.8 a). The variation of $G$ causes a response of $u_{i n}$ which leads to the observed $T$ variations during the cast. However, Equations (2.5), (2.11) and (2.15) predict a similar decrease in $L$ during the cast, as shown in Figure $2.8 \mathrm{~b}$ ). A constant value of $V=0.12 \mathrm{~m} / \mathrm{s}$ is used to predicted $L$. While we do observe an initial decrease in the experimental data for $L(t)$, the turning and following increase in $L$ cannot be predicted based on the change of $\bar{G}$ alone. The changing $V$ must also be considered to explain $L(t)$.

\subsubsection{Solidification velocity}

The experimental results from Section 2.2.2 indicate that $V$ is not constant through the cast. Note that $V$ is not a control parameter for the process, rather it is a 

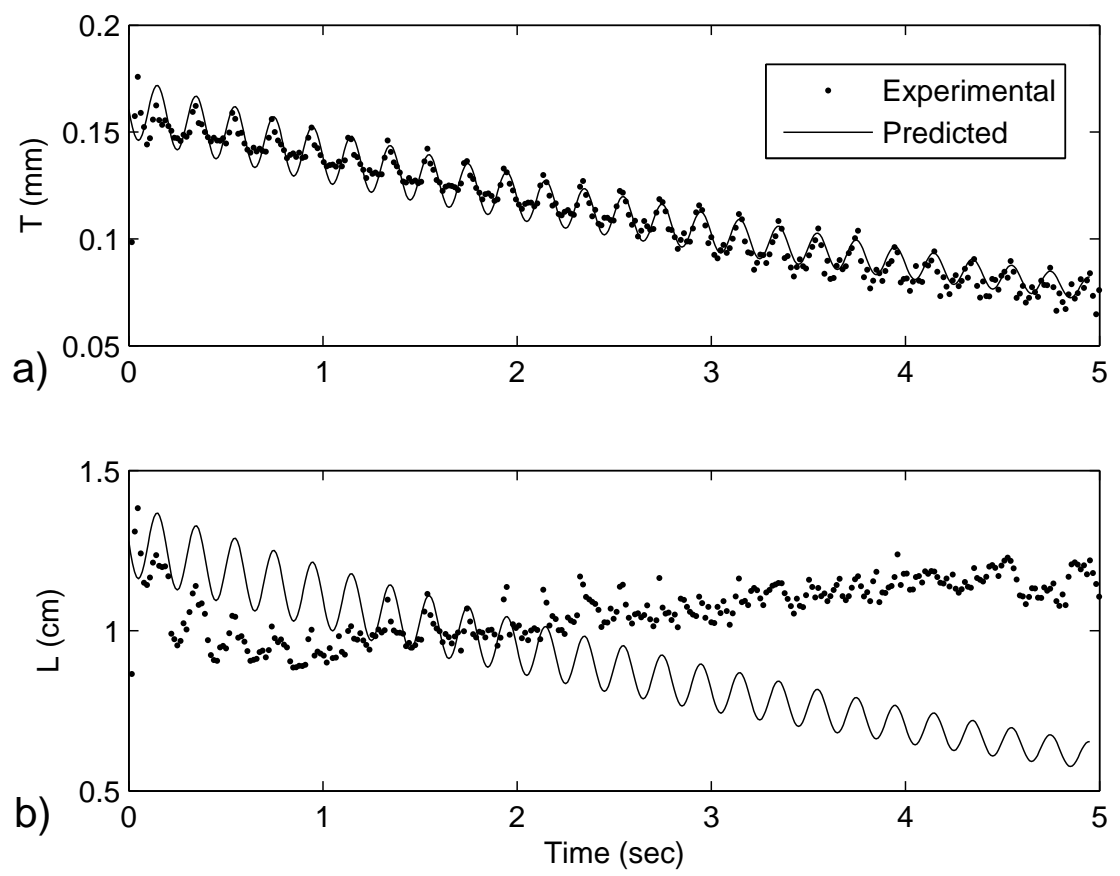

Figure 2.8: Predictions for $T$ and $L$ based on the steady state mass balances and the Bernoulli equation for coupling $G$ and $u_{i n}$. a) The predicted $T$ agrees well with the experimental values. b) The predicted $L$ (using $V=0.12 \mathrm{~m} / \mathrm{s}$ ) shows a steady decrease, similar to $T$, rather than the increase that is observed experimentally. This indicates that $V$ is not constant throughout the cast. (Data from cast ODSU06-22) 
process output. In general, $V$ is a function of many operating parameters, including but not limited to, wheel temperature $(\Theta)$, the condition of the wheel surface, melt superheat $\left(T_{h}\right)$, and the final ribbon thickness $(T)$. We can infer $V$ from the steady state mass balance, which gives $V=T U / L$. Since $T, U$ and $L$ have been measured throughout the cast, we can get a direct measurement of how $V$ varies with time. Note from Figure 2.2 that $T$ decreases as the cast progresses even while $L$ tends to increase, indicating that $V$ must be decreasing during the cast. Figure 2.9 shows how $V$ changes with time, decreasing from $0.14 \mathrm{~m} / \mathrm{s}$ to $0.06 \mathrm{~m} / \mathrm{s}$ during the cast. A linear regression is applied to the data to give

$$
V(t)=V_{i}-m t
$$

where $V_{i}=0.136 \mathrm{~ms}^{-1}$ is the initial solidification velocity and $m=0.0184 \mathrm{~ms}^{-2}$ is the rate at which $V$ decreases. It is observed experimentally that $V$ decreases similarly from cast to cast and the empirical for $V(t)$ can be applied to other casts.

Many of the experimental parameters are evolving with time, making it difficult to identify the primary cause of the decrease in $V$. Perhaps the most relevant parameter, $\Theta$, increases from 30 to $100{ }^{\circ} \mathrm{C}$ during the cast. However, it is not clear how the increase in $\Theta$ may influence the surface of the wheel and thus effect the contacting and heat transfer of the process. A further discussion of the physical significance of the variation in $V$ is given in Section 2.5.

\subsection{Results}

A dynamical model for the time dependent behavior observed in our experimental casting has been developed. The model involves iterating between two calculation steps. Initially, the steady values of $T$ and $L$ are determined by combining the 


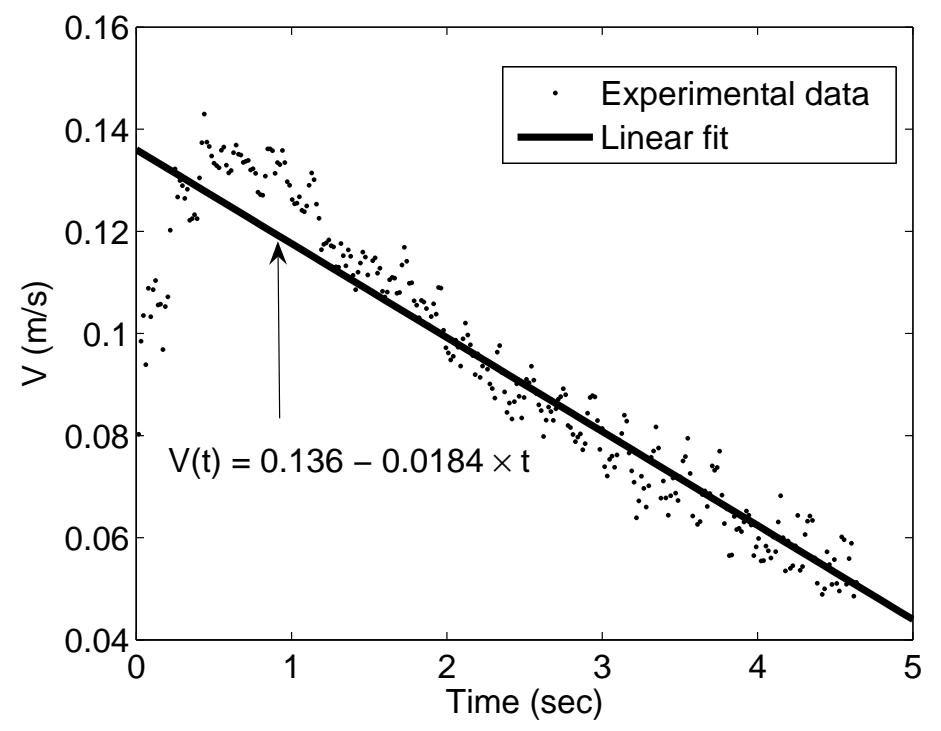

Figure 2.9: The average solidification velocity, $V$, is inferred from direct measurements of $T U / L$ through the cast. A linear fit to the data provides an estimate for $V(t)$.

processing parameters $G_{i}, V_{i}, U$ and $\Delta P$ with Equations $(2.10),(2.11)$ and $(2.15)$. After a small time step, $\Delta t$, the amount of heat that has been transferred to the wheel is calculated, giving an estimate for $\Theta(t)$ and the overall decrease in the gap height $\Delta G$. New values for $G(t)$ and $V(t)$ are then calculated, as given by Equations (2.5) and (2.16) respectively. The calculation steps are then repeated until the end of the cast. The results are insensitive to the size of the time step, $\Delta t$, provided $\Delta t<\tau_{L_{R}}$. That is, the time step of the model should be smaller than the time scale over which the experimental data is averaged.

Figure 2.10 compares the predicted values of $T, \Theta, G$ and $L$ to the experimental values. The empirical models for $\delta(t)$ and $V(t)$ (from cast ODSU06-22) are used in a predictive manner by applying the fits to a different set of cast data (from cast ODSU06-40). The model predicts the periodic variation of $T$ and the overall 
decrease. The predicted value of $\Theta$ is also compared to the experimental measurement. The predicted value is not periodic whereas the thermocouple measurements are because they are fixed in the substrate. The predicted $\Theta$ value is greater than that of the measured value after $\sim 3$ seconds. The volume of the substrate used for the heat-up calculation is based on the width of the ribbon $(5 \mathrm{~cm})$, the circumference of the wheel $(191 \mathrm{~cm})$ and the depth of the wheel $(1.27 \mathrm{~cm})$. However, the width of the substrate $(13.5 \mathrm{~cm})$ is actually greater than the width of the ribbon. Therefore lateral diffusion of heat throughout the wheel will occur over longer times and the differences between the measured and predicted wheel temperatures are anticipated.

The decrease in $G$ predicted by the model is also compared to the measured value in Figure 2.10. Equation (2.5) provides the estimate for $G(t)$ and is used as a mechanical forcing in the dynamical model of the process. The model overestimates the decrease in $G$ near then end of the cast. This is likely due to the predicted value of $\Theta$ being too large, causing the expected thermal expansion to be greater than the observed value. The empirical estimate of $\delta(t)$ based on experimental observation is applied so the decreasing amplitude of the forcing can be observed.

The predicted $L$ is more complicated to interpret. The decreasing $G$ decreases $u_{\text {in }}$ and causes a smaller $L$. However, the decrease of $V$ during the cast is such that $L$ actually increases. Therefore, the overall decrease in $L$ that is anticipated due to the decreased flow rate is not observed. Rather, the changing thermal conditions of the substrate cause $V$ to decrease faster than $u_{i n}$ and an increase in $L$ is observed. The model does not, however, predict the initial behavior of $L$, where it increases in length during the first second of the cast. Data in Section 2.7 is included to 

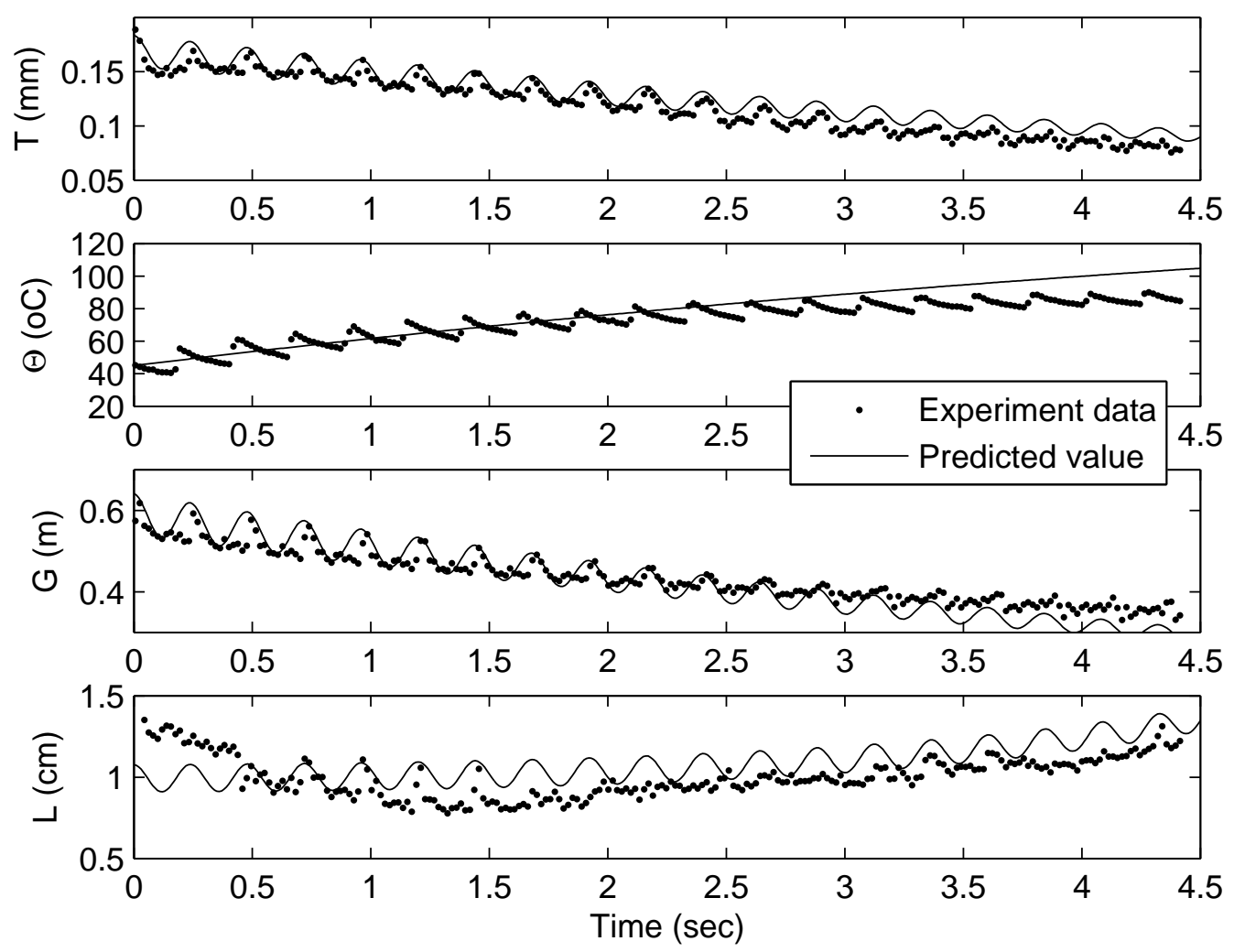

Figure 2.10: Experimental data and the predicted values of ribbon thickness, $T$, wheel temperature, $\Theta$, gap height, $G$, and puddle length, $L$, as a function of time during the cast. $(U=8.0 \mathrm{~m} / \mathrm{s}, \Delta P=6500 P a$ are held constant. Data from cast ODSU06-40) 
show that the dynamic model of the casting can be used to predict the process behavior for varied casting conditions.

\subsection{Discussion}

Our approach in this modeling is to capture the puddle dynamics through control volume balances. The changing parameters $G$ and $\Theta$ are primarily responsible for the transient behavior of the cast. The unsteady mass balances reduce to the steady behavior where $B u_{i n}=\bar{T} U=V \bar{L}$. Fluid flow and thermal effects are incorporated in a quasi-steady manner to give a simple dynamical model of the process. It may be surprising that this method can capture the transient behavior of the casting as well as it does. However, the dynamic mass balances indicate that slowly varying process parameters can be treated as quasi-steady for $t>t_{s}$ (given by $t_{s}=\gamma^{-1}$ from Equation (2.14)). To capture unsteady behavior at smaller scales, such as defect formation ( $m m$ scale) or microstructural variations ( $\mu m$ scale), we would likely need to account for unsteady momentum and thermal effects. It is important to understand these larger scale thickness variations, particularly when considering continuous production of such ribbons.

$T$ is observed to vary throughout the casting process. The out-of-roundness of the substrate provides a mechanical forcing that causes the molten metal puddle to respond by changing in size. Varying the initial $G$ changes the amplitude of the forcing disturbance, set by the initial out-of-roundness of the wheel. That is, as the initial gap height is decreased, the forcing amplitude is increased. Casting many amorphous materials requires a smaller gap spacing than what our experiments require. The wheel out-of-roundness becomes more significant as the gap spacing is further lowered. 
The model of the wheel expansion that we have presented in Section 2.3.1 is clearly a simplification. We assume that the heat transferred to the wheel is distributed uniformly, thereby avoiding any thermal stresses in the wheel. Others have studied the thermal effects during twin-roll strip casting, coupling heattransfer and casting roll deformation (Park et al., 2003) and how thermal stresses develop during rapid solidification on a thin plate (Al-Huniti \& Al-Nimr, 2000). It is interesting to note how the wheel deforms as it is thermally loaded by the ribbon. However, it is beyond the scope of this study to predict the wheel deformation. Equation (2.5) gives the model that is developed for $G(t)$. It consists of the initial gap setting, the out-of-roundness amplitude, $\delta$, the wheel rotation frequency, $\omega$, and $\Delta G$ due to the wheel expansion. To give a better approximation of the experimental results, a linear fit for the decreasing wheel out-of-roundness, $\delta(t)$, is used. Although this is an empirical fit, the wheel deformation seems to be very reproducible from cast to cast.

The variation of $G$ causes a response in both $T$ and $L$. Recall that $\Delta P$ is held constant in the crucible, rather than fixing the flow rate, $u_{i n}$, through the nozzle. Thus, $G$ variations leading to $u_{i n}$ variations. The steady values of $T$ and $L$ are directly proportional to $u_{i n}$ and the time-dependent $G$ can be viewed a a mechanical disturbance to the steady state of the process.

One would expect the opposite trend to occur for constant $u_{i n}$ conditions. As $G$ decreases, $u_{i n}$ cannot decrease in response and the volume of the puddle must remain constant. The puddle must then get longer to accommodate the smaller $G$. As the puddle gets longer, $\tau_{L}$ increases and the ribbon would get thicker. This is the opposite trend that one finds for the fixed $\Delta P$ situation.

It is also interesting to note that $L$ gets longer throughout the cast, even as $T$ 
decreases. The steady state balances indicate the $V$ must therefore be decreasing during the cast. We infer $V$ directly from our experimental measurements of $T, U$ and $L$ through the cast. However, an analysis of the microstructure of the ribbon at different positions along the cast length has not been done. A recent work by Byrne et al., 2007 does examine how the solidification rates varies through the thickness of the ribbon. However, there is no comparison done at different positions along the cast length. Still, the experimental measurements indicate that $V$ evolves over time. We have used an empirical model to predict $V(t)$ during the cast. Since many of the process parameters are varying through the cast, it is not clear how to attribute the decrease in $V$. The increase in $\Theta$ would decrease the total temperature difference between the molten metal and the wheel. However, $V$ decreases by a factor of two during the cast (refer to Figure 2.9). The increase in $\Theta$ only causes the total temperature difference between the molten metal $\left(710^{\circ} \mathrm{C}\right)$ and the wheel $\left(30-100{ }^{\circ} \mathrm{C}\right)$ to change by about $10 \%$, thus the wheel heat-up is not solely responsible for the decrease in $V$.

It is more likely that the contacting conditions between the molten metal and the wheel are changing during the cast. There is evidence from the high speed video that this may be the case. Figure 2.11 shows three high speed video images at different times during the cast and provides further insight on the contacting between the wheel and the molten metal puddle. A high-intensity fiber optic light is used to locally back-illuminate the upstream meniscus (USM). This allows for higher magnification of the USM than when the entire puddle is imaged. The wheel surface acts as a mirror and reflects the high intensity light, making it difficult to determine the position of the wheel. However, a white line is drawn over the image to indicate the wheel surface and the contact line between the USM and 
the wheel. Figure 2.11 a) shows an image from the first revolution of the casting wheel. It appears that dust particles have settled on the wheel surface during the time between the polishing of the wheel and the melting stage of the process. The depth of focus for these images is only a few $m m$, due to the high magnification used in imaging the puddle. For this reason, particles out of the focal distance are blurry and may appear to be positioned either higher or lower than the wheel surface. The ribbon from the first revolution of the wheel is often of poorer surface quality the the rest of the cast. Figure $2.11 \mathrm{~b}$ ) shows the USM and the wheel surface conditions after 4 revolutions of the wheel. No particles are evident on the wheel surface and the ribbon quality improves. This also corresponds to the time during the cast where $L$ reaches its minimum value. Figure 2.11 c) shows the wheel conditions after 15 revolutions of the wheel. It appears that larger particles stick to the wheel. The appearance of these particle occurs gradually during the cast, but becomes increasingly more frequent with time. The source of these particles is not clear. However, the wheel surface is not cleaned during the cast. Also, the oxidation of the $\mathrm{CuBe}$ wheel is not controlled during the cast, as we cast in an ambient environment. The quality of the ribbon generally degrades as the cast progresses (e.g. poor edge quality, longitudinal streaks and small holes forming in the ribbon). While these are qualitative observations, they do indicate that the contacting conditions between the molten metal and the wheel could be in part responsible for the decrease in $V$ during the cast.

The turning behavior in $L$ is difficult to predict. Other studies have considered the startup and ending portions of the cast length as transient and disregarded them (Praisner et al., 1995; Byrne et al., 2006). The initial increase then turning in $L$ would likely be missed and only a steady increase would be observed. It is 

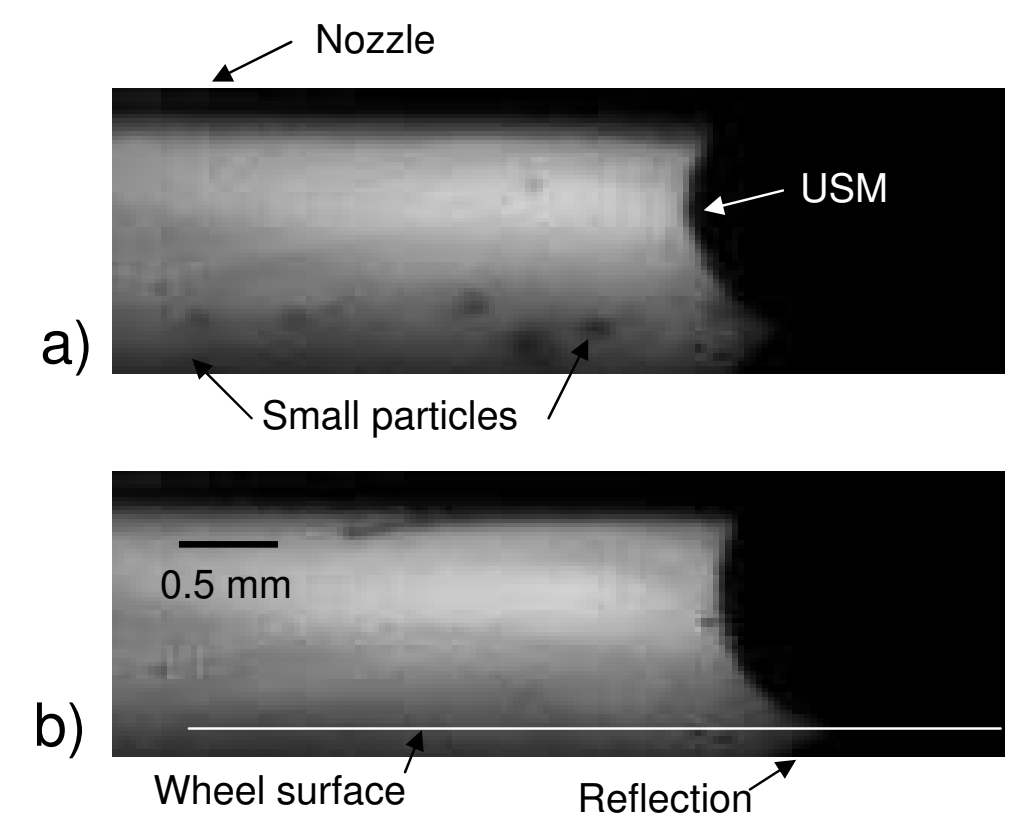

c)



Figure 2.11: Images of the USM and wheel surface at different stages of the cast. A white line is superimposed on the image to indicate the wheel surface. a) Dust particles appear on the wheel surface during the first revolution of the cast. Particles that appear higher than the white line are further back on the wheel surface (out of the focal length) and the particles appearing lower are closer to the camera. b) The wheel surface appears free of particles after $\sim 3$ wheel revolutions. c) After 15 wheel revolutions larger particles commonly appear on the wheel surface. 
also interesting to note that $L$ responds to both the fluid flow (mechanical forcing of $u_{i n}$ ) and the thermal dynamics (substrate heat-up) of the process. Initially, $L$ decreases as one might expect from the decrease in $G . L$ begins to increase after about 1-2 seconds of casting. This increase through the cast indicates a transition where the thermal dynamics seem to have a greater influence on determining the observed $L$ than the fluid dynamics.

A simple explanation for this turning may be due to the finite depth of the casting rim. Based on the thermal diffusion and the rim thickness, a characteristic time scale of $\tau_{w}=W_{D}^{2} / \kappa \approx 1.4$ seconds is estimated. Here $W_{D}$ is the depth of the $\operatorname{rim}(1.27 \mathrm{~cm})$ and $\kappa$ is the thermal diffusivity of the wheel $\left(1.16 \mathrm{~cm}^{2} / \mathrm{s}\right)$. This gives a time scale for the wheel to transition from being semi-infinite sink for heat to that of a solid finite in depth. It also corresponds to the time where $L$ begins to increase and the thermal effects of the wheel start to dominate over the fluid flow.

\subsection{Conclusion}

A dynamical model of the PFMS process has been developed to account for the observed variation in $T, \Theta, G$ and $L$ during a cast. Control volume mass balances which can change in size are used to provide a dynamical model for $T(t)$ and $L(t)$. The process can be treated as quasi-steady for the slowly changing process parameters that influence the flow rate, $u_{i n}$ and the solidification velocity, $V$. The the wheel out-of-roundness provides a mechanical forcing to $G(t)$ and causes the flow rate into the puddle to vary. The wheel heat-up and expansion is calculated and used to estimate $G(t)$ during the cast. Both $T$ and $L$ respond in phase to the periodic forcing of $G(t) . T$ can be predicted from the steady mass and momentum balances alone. $L$, however, depends on both $u_{\text {in }}$ and $V$. During the initial stage 
of casting, the fluid flow seems to determine $L$ and it decreases with $G$. However, the thermal conditions of the wheel dominate after $\approx 1-2$ seconds of casting, after which $L$ steadily increases. An empirical estimate of $V(t)$ is developed from a linear regression of experimental data. The model is compared with experimental results for varied processing conditions.

\subsection{Data appendix}

Equation (2.5) is prescribed to specify $G(t)$ in the dynamical model of the process. However, the measurement of $G$ from the non-contact capacitance gauge can also be used so that no fitting function is needed. Figure 2.12 shows the results for $T(t)$ and $L(t)$, as calculated by applying the measured value of $G$ to Equation (2.15), along with Equations (2.10), (2.11) and (2.16). The predicted value of $T$ more closely matches the experimental value $T$. Note that Equation (2.5) fits $G$ to a sin function of amplitude $\delta$. Using the measured value of $G$ allows for a more accurate prediction of $T$ within each wheel revolution. The predicted and measured values of $L$ also show reasonable agreement, as does that of $\Theta$. Finally, the measured value of $G$ is plotted in Figure 2.12. While using the measured value of $G$ in the dynamical model does provide for better agreement between the predicted and experimental data, it limits the predictive capabilities of the model (i.e. the measured value of $G$ is only available after the cast).

There are two fitting functions that are used to give the dynamic model of the PFMS process. The first is how the wheel out-of-roundness, $\delta$, changes with time. The empirical fit is given by $\delta(t)=\delta_{i}-k t$, with $\delta_{i}=50 \mu \mathrm{m}$ and $k=7.5 \mu \mathrm{m} / \mathrm{s}$ where $\delta_{i}$ is determined from the out-of-roundness of the wheel prior to casting and $k$ is the rate at which the wheel deforms. Modeling the wheel deformation upon 

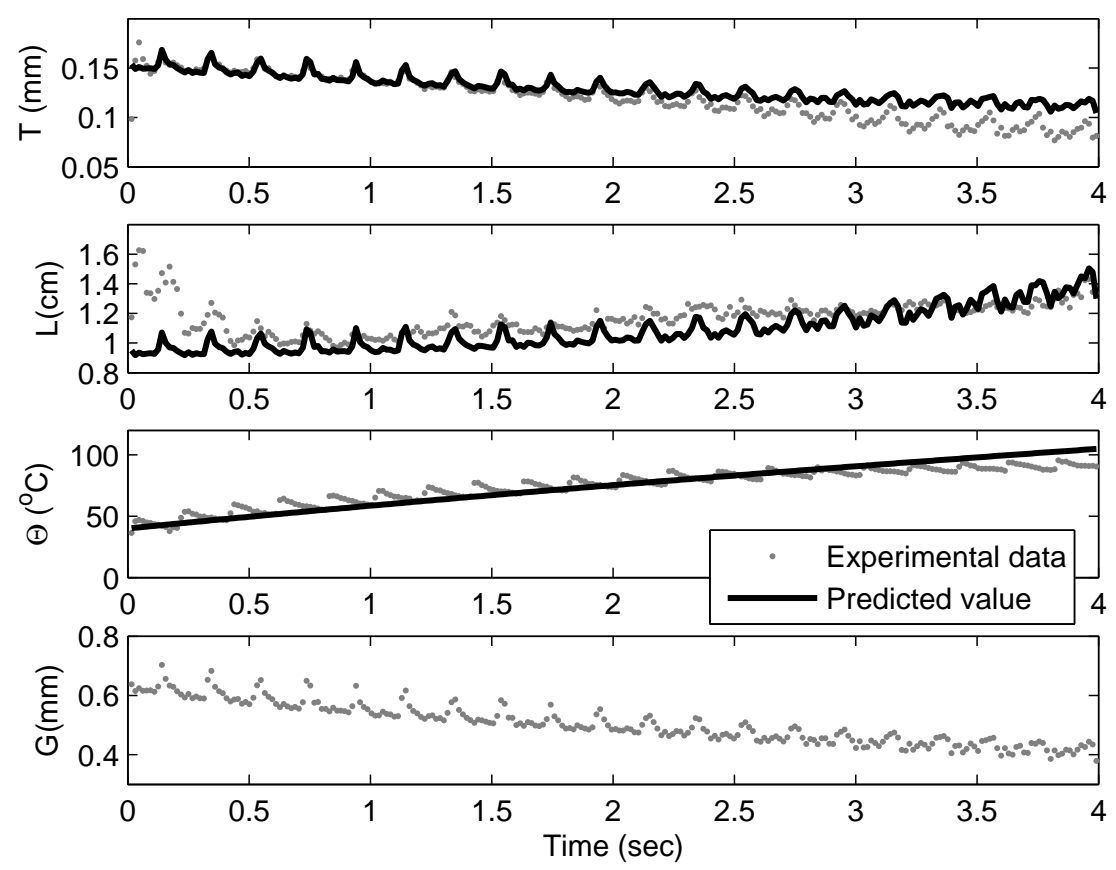

Figure 2.12: Predicted values for $\mathrm{T}(\mathrm{t}), \mathrm{L}(\mathrm{t})$ and $\Theta(t)$ using the $G(t)$ measured by the capacitance gauge in the dynamical system. (Data from cast ODSU06-22). 
heating is beyond the scope of this study and the empirical fit is based on the trends generally observed during the cast.

The second fitting function is for the solidification velocity $V(t)$. A linear regression is applied to experimental data to give $V(t)=V_{i}-m t$, where $V_{i}=$ $0.136 \mathrm{~ms}^{-1}$ is the initial solidification velocity and $m=0.0184 \mathrm{~ms}^{-2}$ is the rate at which $V$ decreases. It is observed experimentally that $V$ decreases similarly from cast to cast and the estimate of $V(t)$ can be applied to other casts. There are many mechanisms by which $V$ may decrease during a cast, including the wheel heat-up, casting in an ambient air environment, oxide formation on the wheel and / or molten metal puddle and dust particles collecting on the wheel surface during the cast.

The fitting functions for $\delta(t)$ and $V(t)$ give a reproducible measure of the process under varied experimental conditions. A model is compared to experimental results in Figures $2.13-2.17$. 

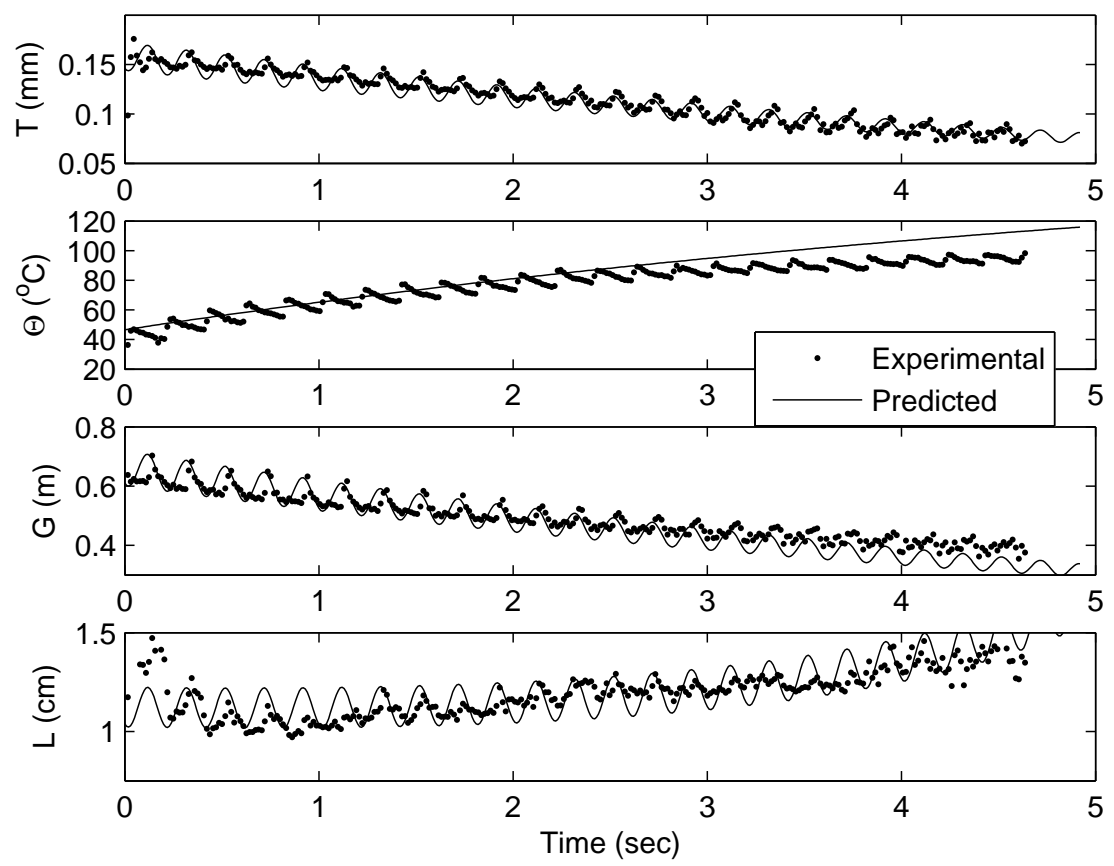

Figure 2.13: Experimental data of ribbon thickness, $T$, wheel temperature, $\Theta$, gap height, $G$, and puddle length, $L$, as a function of time during the cast. The other casting conditions (held fixed) are $U=9.5 \mathrm{~m} / \mathrm{s}$ and $\Delta P=5000 \mathrm{~Pa}$. (Data from cast ODSU06-22) 

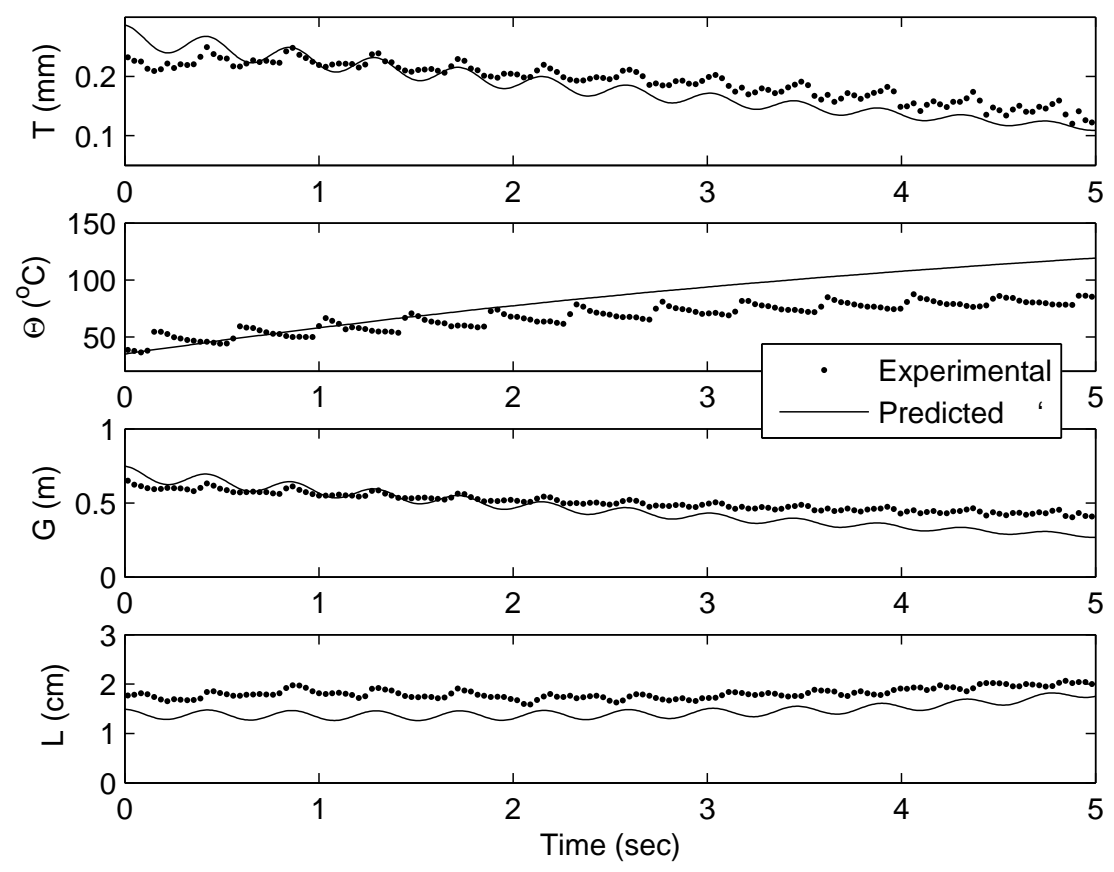

Figure 2.14: Experimental data of ribbon thickness, $T$, wheel temperature, $\Theta$, gap height, $G$, and puddle length, $L$, as a function of time during the cast. The other casting conditions (held fixed) are $U=7.1 \mathrm{~m} / \mathrm{s}$ and $\Delta P=7400 \mathrm{~Pa}$. (Data from cast TBSP06-20) 

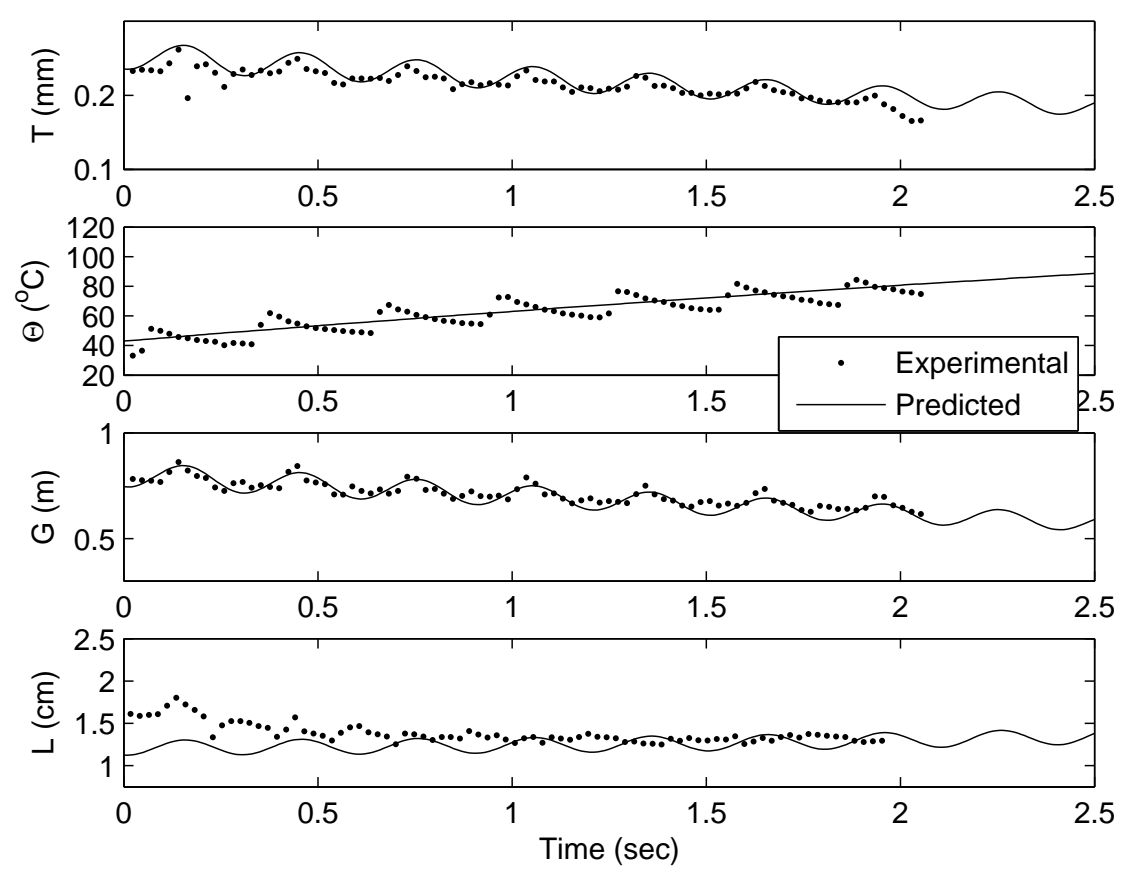

Figure 2.15: Experimental data of ribbon thickness, $T$, wheel temperature, $\Theta$, gap height, $G$, and puddle length, $L$, as a function of time during the cast. The other casting conditions (held fixed) are $U=6.4 \mathrm{~m} / \mathrm{s}$ and $\Delta P=5000 \mathrm{~Pa}$. (Data from cast ODSU06-42) 

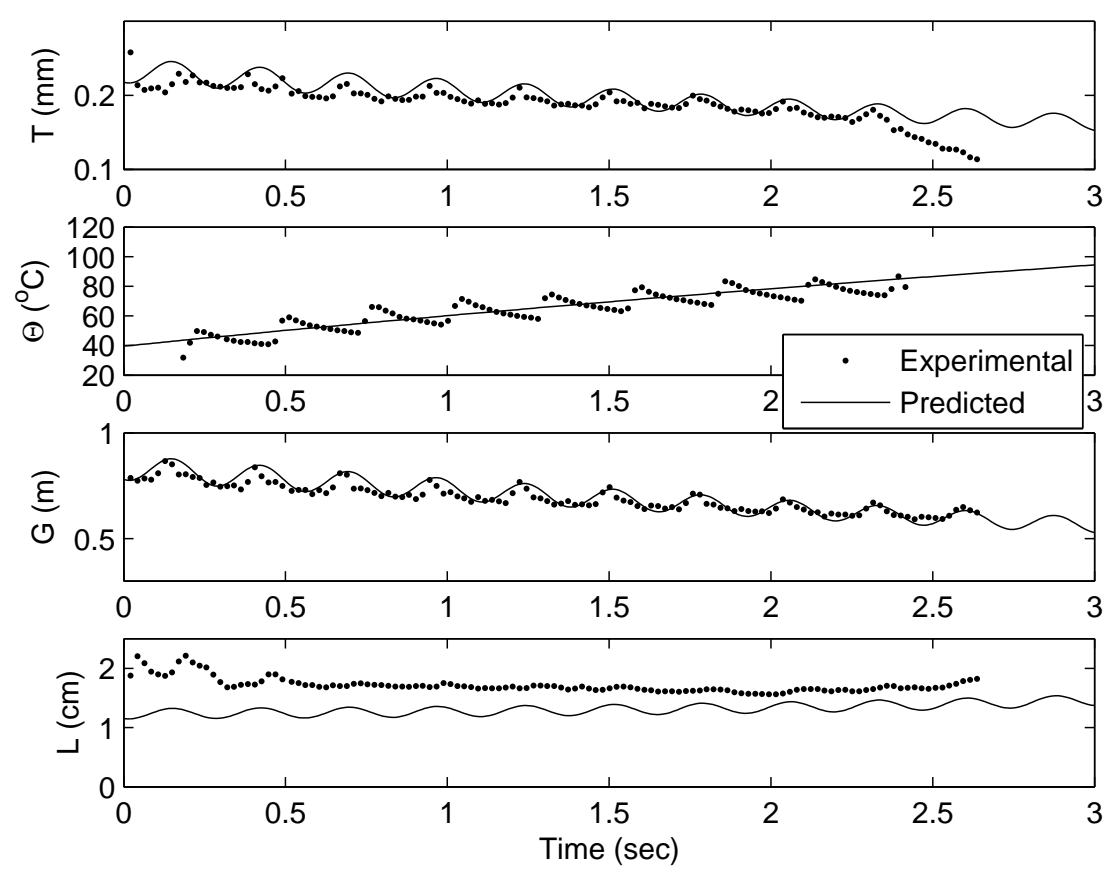

Figure 2.16: Experimental data of ribbon thickness, $T$, wheel temperature, $\Theta$, gap height, $G$, and puddle length, $L$, as a function of time during the cast. The other casting conditions (held fixed) are $U=7.0 \mathrm{~m} / \mathrm{s}$ and $\Delta P=4600 \mathrm{~Pa}$. (Data from cast ODSU06-43) 



Figure 2.17: Experimental data of ribbon thickness, $T$, wheel temperature, $\Theta$, gap height, $G$, and puddle length, $L$, as a function of time during the cast. The other casting conditions (held fixed) are $U=7.1 \mathrm{~m} / \mathrm{s}$ and $\Delta P=5000 P a$. (Data from cast ODSU06-47) 


\section{Chapter 3}

\section{Capillary Puddle Vibrations Linked to}

\section{Casting-defect Formation in Planar-flow}

\section{Melt Spinning*}

\subsection{Introduction}

Planar-flow melt spinning PFMS (also known as planar-flow or single-roll spin casting) is a continuous casting technique for rapidly solidifying thin metal sheets or ribbons. The technique has been of interest (1) because of the economic and environmental advantages of casting a final product directly from the melt and (2) because of the special properties arising from the unusual microstructure of rapidly-frozen alloys. For ribbons cast thinner than 1 millimeter, present benchscale machines, including ours, make product at economically attractive rates but of insufficient quality. Our goal is to develop a scientific understanding of various undesirable features appearing in the product so that these may be eliminated or, at least, manipulated, and the quality thereby enhanced.

We cast aluminum (and $\mathrm{Al}$ alloys) into ribbon of $50 \mathrm{~mm}$ width (not limiting) and $0.1 \mathrm{~mm}$ thickness, typically. The distinction between surface and bulk features blurs at this thinness. A charge of $1 \mathrm{~kg}$ yields a ribbon of nearly 75 meters long at these dimensions. A typical casting speed is $10 \mathrm{~ms}^{-1}$. Note that our casting speed

${ }^{*}$ Reprinted, with permission, from Metallurgical and Materials Transactions B, 36B, C.J. Byrne, E.A. Theisen, B.L. Reed and P.H. Steen, "Capillary puddle vibrations linked to casting-defect formation in planar-flow melt spinning", pp. 445-456, 2006. Sections 3.53 .7 and 3.8 have been added to the original manuscript. 
is an order-of-magnitude faster and our thickness an order-of-magnitude thinner than the state-of-the-art for commercial casting (Nucor, 2002).

The focus of this paper is an imperfection that crosses the ribbon product span-wise with a regular stream-wise spacing. The marks are easily observable on both top and bottom surfaces with the naked eye. The 'cross-stream wave', as it is sometimes referred to, arises over a broad range of conditions and has been reported in other studies (Praisner, Chen \& Tseng, 1995; Haga \& Suzuki, 2003b). It is observed by laboratories casting a variety of alloys. The thrust of this paper is that this wave defect ultimately arises from the oscillation of the molten metal puddle at a natural frequency determined by the balance between liquid inertia and surface tension. Such a mechanism is consistent with the prevalence of the defect. Previous work has speculated about the role of the free surface meniscus in the formation of such features (Wilde \& Matthys, 1992; Napolitano \& Meco, 2004). The present work establishes the link with a particular puddle vibration.

PFMS has been studied extensively over the two decades following 1975, driven mainly by an interest in the enhanced magnetic and mechanical properties that rapidly-quenched materials can have due to their microcrystalline or amorphous microstructures. Early reviews dealt with the material properties of the meltspun product with some focus on processing issues (Kavesh, 1978; Jones, 1982). The fluid flow came into focus later (Huang, 1981). A review of the process fluid mechanics is available (Steen \& Karcher, 1997). More recently, combinations of numerical and experimental studies have appeared (Chen \& Tseng, 1999; Bussmann, Mostaghami, Kirk \& Graydon, 2002). Other work has focused on correlating processing parameters with ribbon quality and dimensions (Haga \& Suzuki, 2003b; Wilde \& Matthys, 1992; Huang \& Fiedler, 1981; Carpenter \& Steen, 1992). Many 
authors have reported surface features including herringbone patterns, dimples, striations in the cast direction and cross-stream waves, but mechanisms of formation have not been identified (Praisner, Chen \& Tseng, 1995; Haga \& Suzuki, 2003b; Carpenter \& Steen, 1992).

Thickness variations in the product cast in our laboratory are seen at various length scales. The cross-stream wave corresponds to typical thickness variations of about $30 \%$ of the mean (for $\sim 150 \mu \mathrm{m}$ thick ribbons). This type of feature is persistent in our cast ribbons (aluminum alloy) and is detrimental to the integrity of the product. With a nominal Al-7\% Si alloy, the presence of the cross-stream wave reduces the ultimate tensile strength (UTS) by $20-30 \%$ on average, for example.

The cross-stream wave is shown in Fig. 1.7. The feature is apparent as a wavy line running across the width of the ribbon in these photographs. The wave has a well defined wavelength $\lambda$, on the order of one centimeter, in the cast direction. A composite image of both the substrate and free-side of a sample of ribbon, made using a MicroXam Interferometric Profilometer, is shown in Fig. 3.1. Two waves appear in this sample of ribbon. The average thickness of the sample is approximately $180 \mu \mathrm{m}$. On the free side (Fig. 3.1(a)) the thickness reduction resembles a continuous trench. The surface between the wave crests is relatively uniform when compared to the depth of the trench. On the substrate side (Fig. 3.1(b)), what appears as a valley in Fig. 1.7(b) is actually a concentration of craters. The crater depth can be as great as $30 \mu \mathrm{m}$ for this sample. A small number of randomly distributed craters of lesser depth may also be present between waves on the substrate side. However the surface between defects is relatively flat. The defect is in registry between the substrate and free side. That is, there is no measurable 


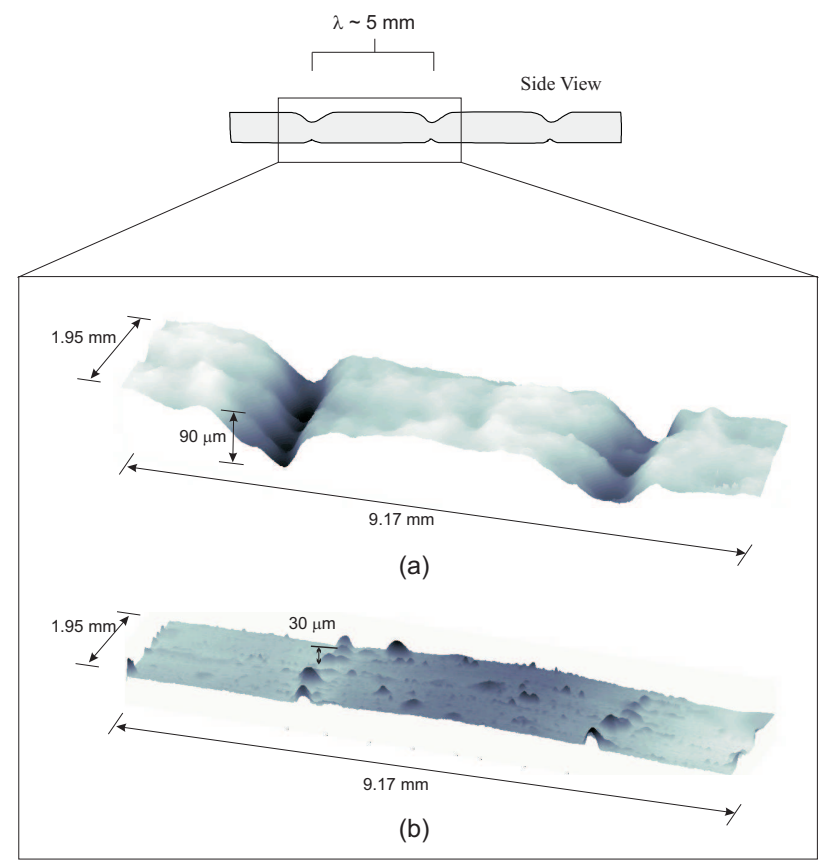

Figure 3.1: Profile of ribbon surfaces (a) Scan of the free-side showing two crosswaves. (b) Scan of substrate-side. Schematic on top shows location of the scans on the ribbon. The craters and trenches are extending into the solid product. Notice that vertical lengths have been expanded for clarity.

horizontal displacement of the trench on the top with respect to the row of craters on the bottom.

A variety of similar surface features have been reported in other continuous casting processes. The origin of short wavelength thickness variations in melt overflow casting of aluminum has been discussed (Legresy, Brechet \& Menet, 1997). These variations have been interpreted as being a result of repeated meniscus pinning/depinning. Also the presence of substrate velocity-dependent cross-stream defects in melt drag twin roll casting has been attributed to the bouncing of the meniscus at the nozzle tip (Haga \& Suzuki, 2003a). The appearance of so called 'oscillation marks' on continuously cast steel slabs is well documented. The frequency 
at which these oscillation marks appear is directly related to the mold oscillation frequency. It has been suggested that several mechanisms may act together to form the oscillation marks, including meniscus freezing and overflow as well as thermal stresses in the solidifying material (B.G. Thomas \& Ho, 1997; Badri, Natarajan, Snyder, Powers, Mannion, Byrne \& Cramb, 2005). In most instances the marks are removed by shaving the finished product.

Periodic cross-stream waves are also seen in many liquid film coating processes. Among these processes, slot coating stands out as having a geometry and operating configuration similar to PFMS. Key differences with PFMS are 1) that solidification occurs downstream of the puddle in typical coating flows and 2) that viscosity plays a dominant role in many coating operations whereas, in PFMS, the high speed of material deposition makes the flow largely inviscid. Nevertheless, it is well-recognized that meniscus and wetting-line stability influence the surface of the coating products (Weinstein \& Ruschak, 2004). Cross-stream periodic bands are observed for a variety of coating processes. These bands are often attributed to the movement of the wetting line (Coyle, Macosko \& Scriven, 1990). Even though it is not known to what extent puddle oscillations are related to any observed coating flow instabilities, it is instructive to use coating flows for contrast.

In Section 3.2 we distinguish two formation mechanisms for periodic features frozen into the cast product. Section 3.3 describes the process and parameters in detail. In Section 3.4 we provide the experimental evidence that the crosswave correlates to motions of the menisci. Section 3.5 examines how the upstream meniscus shape deviates from the average profile during its motion. A correlation between the menisci motions and the main processing parameters is given in Section 3.6. A competition between capillary and inertia effects sets the time-scale for 
the motions. Section 3.7 considers a model problem related to the capillary vibration of the puddle menisci. Section 3.8 discusses the defect formation mechanism. In Section 3.9 we briefly discuss parameter regimes where the cross-wave is not observed. Finally, Section 3.10 compares to the oscillation of an inviscid sphere and a meniscus moving in a channel between parallel plates, related observations from the literature. Additionally, we propose that heat-transfer interruptions are a likely mechanism by which puddle vibrations turn into thickness variations in the product.

\subsection{Template- vs. pulse-transfer}

For any feature with a characteristic stream-wise wavelength on a continuous cast product, one can distinguish two fundamentally different mechanisms of formation. In the first, a pattern on the substrate is transferred directly onto the solidified product, much like what occurs in contact printing where inked type is pressed against a paper substrate to produce a printed page. In this case, the spacing of the template (substrate) is transferred directly to the product. Double the speed of the substrate and the spacing of the lines on the printed page remains unchanged. For a substrate moving at speed $U$, the wavelength $\lambda$ does not depend on $U$. We shall call this template-transfer formation. In the second mechanism, the wavelength is created by a constant-frequency oscillation in the lab frame, much like what would occur by inkjet droplets impinging on a moving substrate from a source of fixed frequency. Doubling the substrate speed will double the wavelength of the feature since the substrate moves twice as far between impingement events. In other words, $\lambda$ is linear in $U(\lambda \sim U / f)$. We shall refer to this as pulse-transfer 
formation. The dependence or not of $\lambda$ on wheel-speed will be key to identifying the physics of cross-wave formation.

A distinct issue is how to report the measured $\lambda$ of periodic features. It can be reported directly as $\lambda$ or as an inferred frequency

$$
f_{\lambda} \equiv U / \lambda
$$

These are entirely equivalent measures, of course. But it should be kept in mind that for different measures the dependence on wheel speed is different for the same formation mechanism. For example, for template-transfer, $f_{\lambda}$ is linear in $U$ while $\lambda$ is independent of $U$ and, for pulse transfer, vice-versa. We shall mostly report data in terms of $f_{\lambda}$, except for the final correlation over a large range of cast ribbons in which case we shall use $\lambda$.

\subsection{Apparatus and experimental methods}

Refer to Fig. 1.1(a) for a schematic of the casting apparatus, which comprises a crucible for housing the molten metal, a nozzle through which the metal can flow and a metallic substrate (or chill wheel). An inert gas pressurizes the crucible in a way that compensates for the decreasing metallo-static head as the metal flows out. Our experimental caster operates using a $1 m$ diameter $\mathrm{Cu}$-Be substrate. A blow-up of the the region between the nozzle and substrate, or contact zone, is shown in Fig. 1.1(b) (horizontal lengths are compressed for clarity). The process feeds molten metal from the crucible (due to a combination of head and applied gas pressure) through the nozzle of breadth, $B$, into the thin gap region between the nozzle and substrate. The pressure at the nozzle entry to the puddle $\Delta P$ is held constant during a cast by the pressure compensator mentioned above. Because of 
the small gap $G$, the nozzle interferes with the flow and a puddle, held by surface tension, is formed. The contact between the substrate and molten puddle is such that enough heat is removed from the melt for solidification to occur. Material solidifies along a front at an average velocity $V$. The substrate (moving with linear velocity $U$ ) continually removes the solidified product from the melt puddle. The final ribbon thickness $T$ depends mainly on the five parameters $B, G, \Delta P, V$, and $U$. Mass, momentum and energy balances with appropriate boundary conditions have been written down and put in non-dimensional form elsewhere (Carpenter \& Steen, 1997), accounting for the effects of the parameters listed in Table 1.1. It is learned that, for operating parameters typical of our experiments, the momentum transfer problem depends on the heat transfer problem but not vice-versa (one-way coupling). The thermal control parameters for the experiments reported in this paper are fixed; only the mechanical control parameters are varied and the influence on the mechanical problem is parametric through $V$ and indirectly through $G$ (it change with time due to wheel heat-up). The experimental casting apparatus in our laboratory is described in detail elsewhere (Carpenter \& Steen, 1992; Kahn, 2000; Reed, 2001).

The range of typical values for processing parameters and variables are given in Table 1.1. Successful casting occurs only for certain 'stability windows' within parameter space. Although steady behavior does not depend on surface tension $\sigma$, the limits of steady behavior ('stability window') do. All data reported below came from experiments carried out in these ranges. Puddle length $L$ and solidification rate $V$ are included as process variables, but they are not actually set by the operator. Specific cast data can be found in the Appendix.

Fig. 3.2 shows the ribbon thickness $T$, wavelength of the cross-wave $\lambda$, and gap 

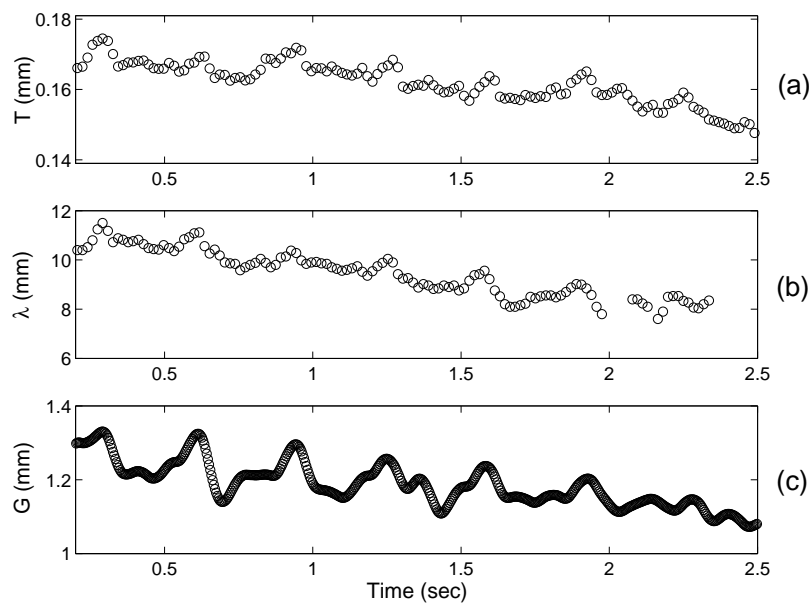

Figure 3.2: Evolution of (a) ribbon thickness,(b) defect wavelength and (c) gap spacing through a cast with $\Delta P$ held constant. Note the gradual decrease in these values due to thermal expansion of the substrate. The periodic behavior is due to the out-of-roundness of the substrate. A 5-piece moving average has been applied to the data for clarity.

spacing $G$, as they vary throughout the duration of a cast. This particular cast is some $20 \mathrm{~m}$ long. Each thickness and wavelength data point represents a value found from averaging over a $0.15 \mathrm{~m}$ length, obtained as follows. The ribbon is cut into a number of $0.15 \mathrm{~m}$-long pieces. For each piece the thickness is measured from the mass and area (reported in Fig. 3.2(a)) and the average wavelength by dividing the $0.15 \mathrm{~m}$ length by the number of waves present (reported in Fig. 3.2(b)).

Fig. 3.2(a) and (b) indicates variations in thickness and wavelength that occur on two length scales larger than the piece size. First, over the total length of the cast (the largest scale), there is a gradual downward trend. Second, there is a periodic variation on the length-scale of the wheel's circumference (about 3 meters). The gradual decrease in thickness has been shown to be the result of wheel heatup. The wheel average heat-up has been measured at about $50-80^{\circ} \mathrm{C}$ for typical 
5 second casts (Kukura, Ford, Singh, Steen \& Ibaraki, 1995). Wheel expansion decreases the gap $G$, increasing the hydrodynamic resistance to flow and hence decreases the flow rate, in view of the constant applied pressure drop $\Delta P$ (Kahn, 2000). The shorter-scale variation shown in Fig. 3.2(a) and (b) is directly related to the out-of-roundness of the substrate, which changes $G$ periodically. Indeed, the period of the oscillation matches the time required for one revolution of the wheel.

The sources of both these long length-scale variations are mechanical and due to time-dependent forcing of the gap. That is, a $G(t)$ is imposed; template transfer occurs. The before-cast hot-gap $G(0)$ is known to within $\pm 5 \%$ of the desired $G$. The change in $G$ throughout a cast is measured by using a run-out meter placed on the substrate. This allows for $G(t)$ to be measured to within $\pm 5 \%$. One such $G(t)$ is shown in Fig. 3.2(c). Both a gradual decrease and periodic variation on this trend are evident in this data. Note that values presented in Fig. 3.2 represent a moving average over $\sim 0.07 \mathrm{~s}$ (five ribbon pieces), done to smooth very local variations.

We will report both gap $G$ and wavelength $\lambda$ values in this study. A series of instantaneous values taken at subsequent instants throughout the cast will be referred to as point-wise data while averages of such a series over the entire cast will be called cast-averaged values. Values of $G$ reported below are 'cast-averaged' unless noted as 'point-wise'. An example of point-wise values is given by the data points in Fig. 3.2(b) and (c). It is impractical to measure $G(t)$ directly for every cast so a means was developed to infer the shrinkage over the duration of a cast as described in the Appendix. This allows us to apply an average decrease to 
subsequent experiments carried out under similar conditions. The error introduced by this approximation is $\pm 10 \%$.

\subsection{Puddle oscillations}

Video imaging of the puddle indicates that the upstream (USM) and downstream menisci (DSM) are moving throughout the entire cast. Motions are captured by a Kodak EktaPro high-speed video system. A high-intensity fiber optic light is used to locally back-illuminate the desired meniscus. The high-speed video system, capable of recording at up to 6000 frames per second, is focused on the profile of the meniscus region. Focusing on the menisci rather than the entire puddle region has allowed much smaller motions of the menisci to be observed than previously reported (Wilde \& Matthys, 1992; Reed, 2001; Ibaraki, 1996). Several regimes of motion of the menisci are identified from these images. These are shown schematically in Fig. 3.3, along with still images of the USM and DSM taken from video. The motions are distinguished as follows.

$\left(A_{1}\right)$ A large scale left-right mean motion of the USM. That is, the mean position of the meniscus moves. This mode is the slowest of those observed.

$\left(A_{2}\right)$ For any mean position of the USM, there are time-dependent bulk deformations superposed on the mean. These are also left-right in nature, but faster than $A_{1}$.

(B) Traveling waves on the DSM, similar to those reported previously (Wilde \& Matthys, 1992).

In Fig. 3.3 only a portion of the DSM is shown. These traveling waves originate at the nozzle-liquid-air contact line (top) and travel towards the liquid-solid-air tri-junction (below). In all observed cases their amplitude decreases as they travel 


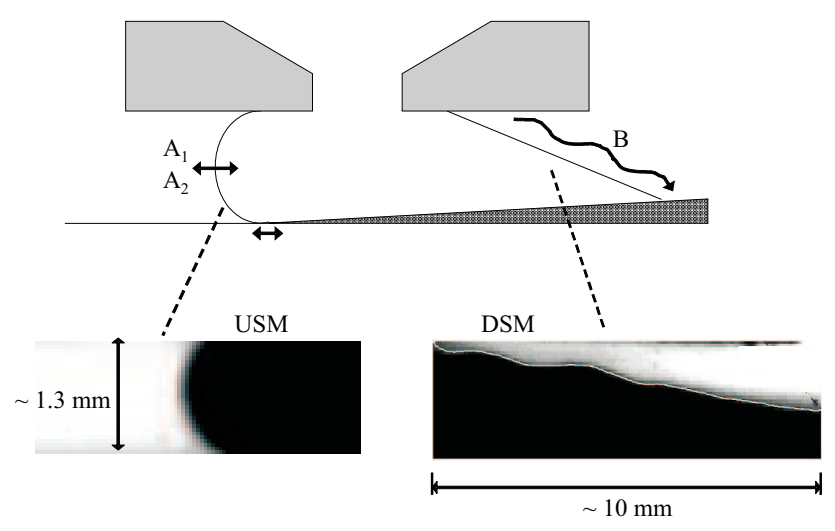

Figure 3.3: Types of puddle motions. $A_{1}$ is a large scale left-right motion of the USM, while $A_{2}$ is a smaller scale left-right vibration of the USM. $B$ represents waves on the DSM. A white line is fitted to the edge of the DSM for clarity. Notice the different length scales associated with the photographs.

downwards. This is consistent with solidification being completed within the puddle region by the time the last molten metal reaches the liquid-solid-air tri-junction.

As seen in Fig. 3.4 the USM is asymmetrical about the mid-plane, being dragged under by the moving substrate. Visualization of the motion of the lower dynamic contact line has had limited success due to the difficulty in illuminating the inner contact region. Fig. 3.4 shows images of the USM over one period of the oscillation illustrating motions of the type $A_{2}$. Notice how the shape of the USM deforms or 'wobbles' as the liquid-substrate contact line pulls to the right (in the direction the substrate is moving).

To quantify these motions, a time series of the displacements of the puddle menisci are extracted from the high-speed video (a threshold technique with linear interpolation yields sub-pixel resolution (Lowry, 1996)). and analyzed for frequency content by fast fourier transform. For brevity we only present data here 

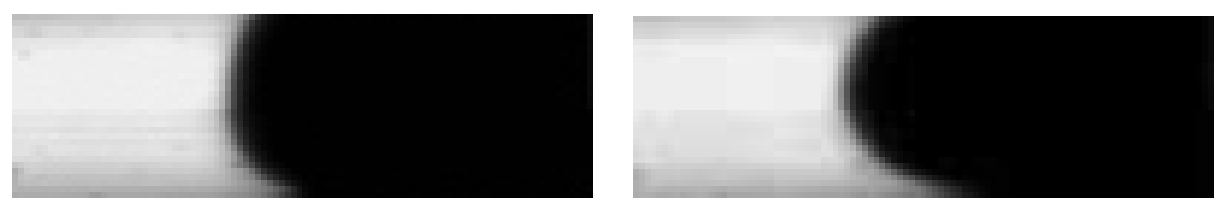

$$
t=0
$$



$t=0.16 \mathrm{~ms}$

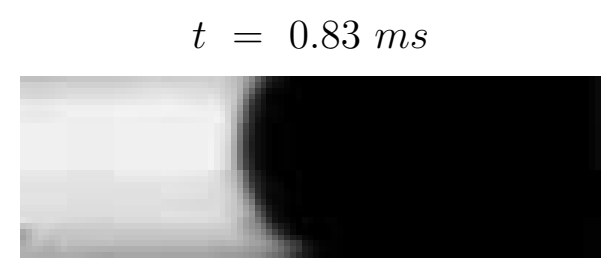

$t=0.33 \mathrm{~ms}$

$$
t=1.0 \mathrm{~ms}
$$
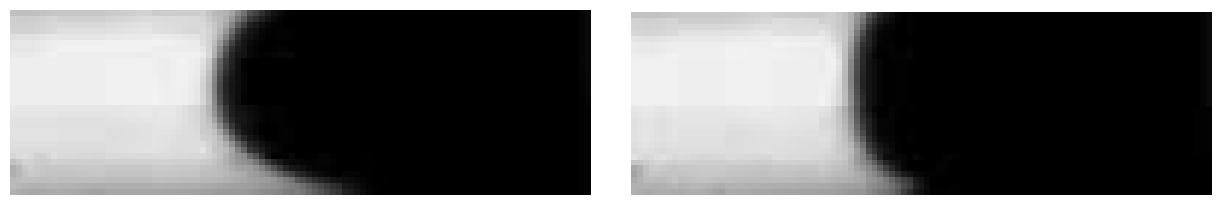

$$
t=0.5 \mathrm{~ms}
$$

$$
t=1.16 \mathrm{~ms}
$$

Figure 3.4: Images of the USM motion over 1 period of oscillation. Meniscus is initially seen to be flat $(t=0 \mathrm{~ms})$ then deforms as contact line moves in the direction of the translating substrate. Eventually contact line moves back to approximately its original position $(t=1.16 \mathrm{~ms})$. The gap spacing is approximately $1.3 \mathrm{~mm}$. 
that relates to motions of type $A$ in Fig. 3.3. Frequencies for motions $B$ are similar to those for motions $A$.

A typical time series of the motions $A$, for $G=1.3 \mathrm{~mm}$, is shown in Fig. 3.5(a). This plot is of the motion of the left-most point on the USM relative to the right edge of the image, which is fixed in space. Motions of different amplitudes are seen. The longest periodic motion shows approximately 9 large peaks over a one second period (motion $A_{1}$ ). Superimposed on this is a smaller amplitude and higher frequency motion, which is enlarged in Fig. 3.5(b)(motion $\left.A_{2}\right)$. On the vertical axis, one arbitrary unit corresponds to approximately $30 \mu m$ of motion of the puddle interface. Note that Fig. 3.5(a) shows 9 peaks during 1 second while Fig. 3.2 shows only 3 peaks. The data come from casts at essentially the same wheel speed. The difference is the moving-average applied to the data in Fig. 3.2; it is done over 5 pieces $(0.75 \mathrm{~m})$ or about one-quarter of the wheel circumference. Thus, since every hill has an adjacent valley and the averaging window always includes a hill/valley pair the smaller 2 of the 3 hills on the wheel get averaged out in Fig. 3.2.

The power spectrum of the data in Fig. 3.5(a) is shown in Fig. 3.5(c). The data were taken to capture the higher-frequency oscillation $f_{2}$ at about $800 \mathrm{~Hz}$. The lower frequency $f_{1}$ just shows up, nevertheless, at about $9 H z$. It has an amplitude several orders of magnitude greater than $f_{2}$. This is in agreement with observations from the raw time series. A slight anomaly is just visible around $1600 \mathrm{~Hz}$. This is the second harmonic of $f_{2}$. The power spectra for motion $B$ show identical peaks at $f_{1}$ and $f_{2}$.

A series of experiments were carried out using different values of $G$ while keeping $U$ fixed (Table 3.1). It is observed that as $G$ is changed the value of $f_{1}$ remains 

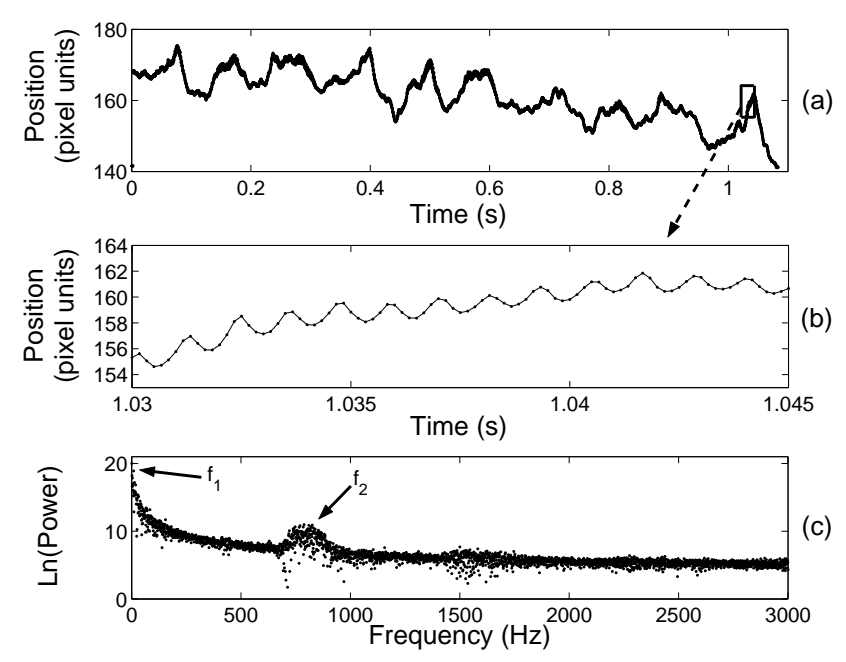

Figure 3.5: (a) Times Series of USM motion (type $A$ ) for $G=1.3 \mathrm{~mm}$. On the vertical axis one unit corresponds to approximately $30 \mu \mathrm{m}$ of motion. (b) Expanded view of smaller portion of data shows the small-scale motions (c) Power spectrum of data (notice log scale on y-axis).

constant. In contrast $f_{2}$ varies with $G$. Also included in the table are corresponding wave frequencies, $f_{\lambda}$, as inferred from the cast-averaged $\lambda$ measurement. Since the gap varies continuously, the frequency also varies along the length of the ribbon, and this non-stationary behavior gives an error associated with $f_{\lambda}$. The reported $f_{\lambda}$ is the average value \pm the standard deviation. The secondary frequency $f_{2}$ appears for all gap heights exceeding $G=0.53 \mathrm{~mm}$. For $G=0.53 \mathrm{~mm}$, no cross-stream waves were present on the ribbon.

It should be noted that the peak centered about $f_{2}$ in Fig. 3.5(c) is rather broad. This is the case in all power spectra from which the $f_{2}$ values reported in Table 3.1 were taken. The out-of-roundness of the substrate is responsible for the broadening of this peak (this can be confirmed using Eqn. 3.3 in the next section). The salient feature is the maximum value (reported as $f_{2}$ ). The values of $f_{2}$ and 
Table 3.1: Summary of $f_{1}, f_{2}$ and average cross-stream wave frequency $\left(f_{\lambda} \equiv U / \lambda\right)$ for different $G$ values.

\begin{tabular}{cccc}
\hline$G(\mathrm{~mm})$ & $f_{1}(H z)$ & $f_{2}(H z)$ & $f_{\lambda}(H z)$ \\
\hline 0.53 & $\sim 10$ & - & - \\
0.78 & $\sim 10$ & 1400 & 1400 \\
1.1 & $\sim 10$ & 950 & 950 \\
1.3 & $\sim 10$ & 800 & 800 \\
\hline
\end{tabular}

$f_{\lambda}$ are nearly the same and consistent with the common source of broadening and have similar standard deviations.

\subsection{Puddle shape deviations*}

The high speed video images can be used to determine the shape deviations of the USM. The procedure that is used is to find the USM profile for a sequence of USM images is shown schematically in Figure 3.6. An averaged profile (solid line) over the sequence is found and each instantaneous image (dashed line) is then compared to the average, shown in Figure 3.6 a). By subtracting the profile of each image from that of the average, an USM deviation is found, shown in Figure 3.6 b). For example, a straight line on the y-axis would indicate no deviation from the average shape. Figure 3.7 shows the results from averaging over 30 sequential images. The units of the axes are pixel units (one pixel unit corresponds to approximately $50 \mu \mathrm{m}$ in length). It is observed that there are two nodes in the interface deviation and the deviation is roughly symmetric about the y-axis.

There are a number of experimental observations which are important to note

*Section 3.5 has been added to the original manuscript. 


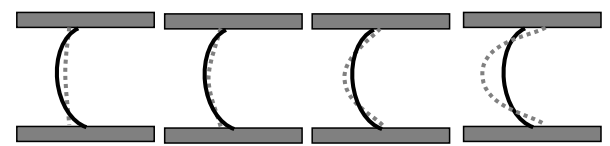

a) Symmetric Vibration of USM

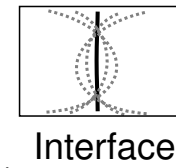

b) Deviation

Figure 3.6: Schematic of the interface deviation during the USM vibration where a) compares each instantaneous image (dashed line) to the average profile (solid line) during one half period of motion and b) subtracts the two to reveal how the shape deviates from the average profile.

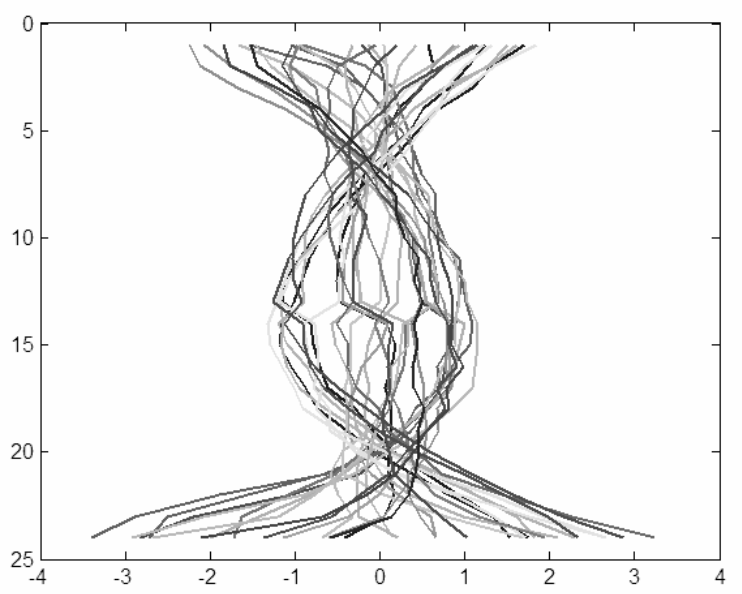

Figure 3.7: The interface deviation from the average USM shape, showing two nodes in the motion. 
before attempting to the model this vibration. First, the vibration frequency is independent of all operating parameters except the gap spacing. For example, if $U$ is doubled we find that $\lambda$ also doubles, thus keeping $f_{\lambda}$ constant. Second, the vibration frequency does not appear to damp throughout the cast. Either the puddle is being continuous perturbed or there is very little dissipation in the puddle. Third, the shape deviation appears to have two nodes in the profile. Finally, the image analysis reveals that both the top and bottom contacts vary during the vibration. It should be noted that contact angle data is difficult to analyze from the high speed video.

Experimental observations that $f_{\lambda}$ is independent of the flow rate in the puddle (i.e. the imposed $\Delta P)$ suggest that the base flow has little effect on the meniscus vibration. Similarly, since $f_{\lambda}$ is independent of $U$ (and therefore also independent of ribbon thickness) the solidification occurring in the puddle has little influence on the observed vibrations. These are certainly qualitative observations, but they indicate that these factors may be neglect to first approximation when considering models for the puddle vibration.

\subsection{Cross-wave physics}

The lower frequency $f_{1}$ relates directly to the out-of-roundness of the substrate. Based on the characteristic substrate speed of Table $3.1(\sim 8.8 \mathrm{~m} / \mathrm{s})$ and the known topography of the substrate, a periodic gap variation of approximately $9 \mathrm{~Hz}$ is expected. Experiments at different wheel-speeds are shown in Table 3.2. Note that $f_{1}$ increases linearly as the substrate-speed is increased. Comparing $U$ and $f_{1}$ values indicates that the wavelength of the topographical features on the substrate is approximately $1 \mathrm{~m}$ corresponding to three hills per circumference; this has been 
verified by direct measurement of wheel topography. It is apparent that the puddle is oscillating at this frequency to accommodate the gap variation. Therefore, frequency $f_{1}$ reflects a template-formation mechanism.

Table 3.2: Behavior of frequency $f_{1}$ as the wheel speed $U$ is varied.

\begin{tabular}{ccc}
\hline$G(\mathrm{~mm})$ & $U(\mathrm{~m} / \mathrm{s})$ & $f_{1}(\mathrm{~Hz})$ \\
\hline 1.0 & 14 & 14 \\
0.8 & 11.9 & 12 \\
0.8 & 8.8 & 9 \\
0.8 & 5.7 & 6 \\
\hline
\end{tabular}

We turn to the frequency $f_{2}$. As shown in Table 3.1, values for $f_{2}$ coincide with the measured cross-stream defect frequency $f_{\lambda}$. That is,

$$
f_{\lambda}=f_{2} .
$$

This frequency increases with decreasing gap height $G$. In contrast Table 3.2 shows three separate casts with cast-average $G=1.3 \mathrm{~mm}$ and where $U$ is varied. It is observed that $\lambda$ varies such that $f_{\lambda}$ remains approximately the same (within the experimental error). We conclude that $f_{\lambda}$ is independent of $U$ and arises from oscillations in the lab frame of fixed frequency. Hence, $\lambda$ and $f_{2}$ result from $a$ pulse-transfer mechanism.

As just described, $f_{\lambda}$ varies with $G$ but is independent of $U$. A log-log plot shows that $f_{\lambda} \sim G^{n}$ where $n \sim-1.42$. To avoid casting failure, parameter variation must remain within the stability window (Carpenter \& Steen, 1992). For this reason, less than a decade of $G$ is tested to determine $n$. Hence, the power law exponent must be interpreted cautiously. To probe how $f_{\lambda}$ changes with other processing 
conditions, 34 casts are compared. Associated conditions are listed in Table 3.3 of the Appendix. Processing conditions vary within the ranges listed in Table 1.1. Cross-wave frequency $f_{\lambda}$ is found not to correlate with $\Delta P, B$ or $W$.

Dimensional reasoning using the observations of the last paragraph suggests that $f_{\lambda}$ be scaled with a capillary time scale $\tau \equiv\left(\rho G^{3} / \sigma\right)^{1 / 2}$, where $\rho$ is the liquid density, $\sigma$ is the surface tension and the characteristic length-scale is chosen to be $G$. That is, generally, one expects $f_{\lambda} \tau$ to depend on the dimensionless control parameters, say, $f_{\lambda} \tau=g\left[\Delta P /\left(\rho U^{2}\right), G / B, G / W\right]$. But observations, including those mentioned above suggest that the function $g$ is in fact a constant, to the accuracy of our measurements. In other words,

$$
f_{\lambda}=C\left(\frac{\sigma}{\rho G^{3}}\right)^{1 / 2}
$$

Here $C$ is a proportionality constant. For a given alloy (fixed $\sigma$ and $\rho$ ), the frequency of capillary oscillations depends only upon $G$. There are two approaches to extracting $C$ from experimental data. The first is to use point-wise values of $G, \lambda$ and $U$ while the second is to use cast-averaged values. It will be shown that these two methods yield the same value for $C$.

Gap $G$ varies throughout the time of the cast and can be measured directly using a run-out meter placed on the substrate, as discussed in Section 3.3. For three different casts, these time-dependent values for $\lambda$ and $G$ are used to plot $f_{\lambda}$ against $\left(\sigma / \rho G^{3}\right)^{1 / 2}$, Fig. 3.8. A linear regression is applied to all the data to yield $C \approx 1.9$. In each of the three casts $G$ decreases by about $30 \%$ throughout the cast consistent with wheel heat-up.

Next, the correlation is tested over a broader range of $U$ and $G$ by cast-averaging 


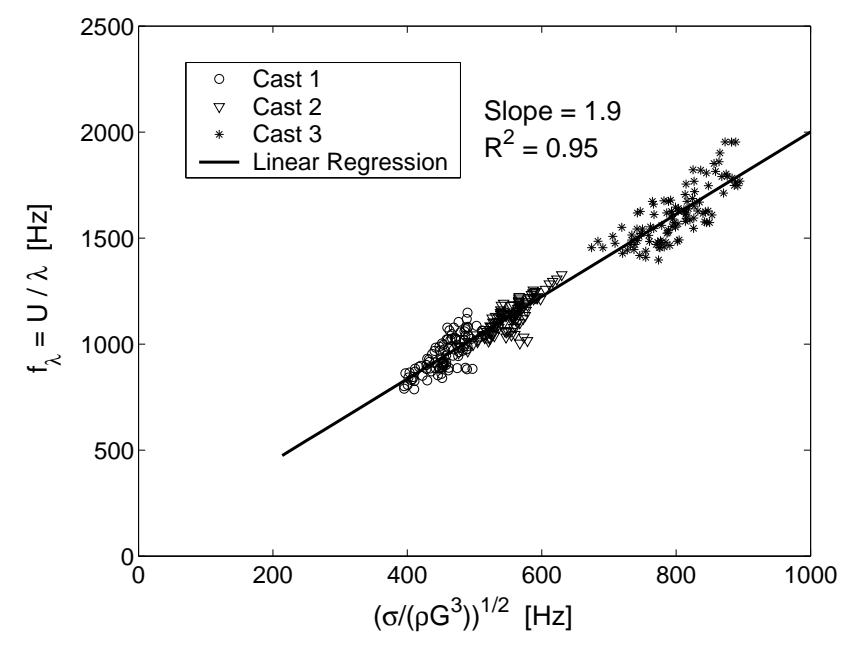

Figure 3.8: Point-wise data. Progression with time is from left to right within each of the three data sets.

( $\approx 4$ seconds) a series of casts. Using Eqn. 3.1, Eqn. 3.3 can be rearranged to yield,

$$
\lambda=\frac{U}{C}\left(\frac{\rho G^{3}}{\sigma}\right)^{1 / 2} .
$$

In Fig. 3.9, 34 separate casts are reported. $G$ is varied between $0.78 \mathrm{~mm}$ and 1.3 $\mathrm{mm}, U$ is varied from $5 \mathrm{~m} / \mathrm{s}$ to $15 \mathrm{~m} / \mathrm{s}, B$ is varied from $1.6 \mathrm{~mm}$ to $3.2 \mathrm{~mm}$ and $W$ is varied from $25 \mathrm{~mm}$ to $50 \mathrm{~mm}$. Each data point represents a cast-averaged value. The main advantage here is that of statistics; each data point represents an average of some 200 point-wise wavelengths (one per piece). The solid line gives the prediction of Equation 3.4. In summary, it is observed that $C=1.9$ correlates the values of $\lambda$ over a broader range of $U$ and $G$ using cast-averaged values than seen in the point-wise correlation of Fig. 3.8. Combined uncertainty in the $G$ and $\lambda$ data is about $\pm 10 \%$. Most data points fall well within this uncertainty and representative error bars are plotted for two data points.

The surface tension $\sigma$ is an important factor in the capillary oscillation of the puddle. By varying $\sigma$, the scaling predicted by Eqn. 3.4 can be further tested. The 


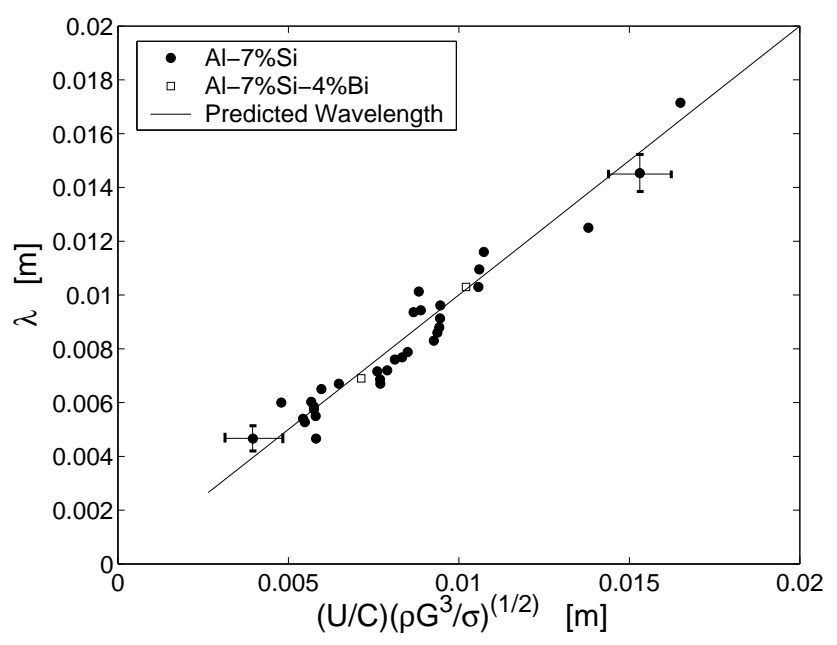

Figure 3.9: Predicted wavelength compared with experimental values. All data falls within the $10 \%$ error bars for cast-averaged $G$.

surface tension of a liquid metal can be varied in two ways: i) by changing the temperature of the liquid and ii) by varying the composition of the material. The surface tension of $\mathrm{Al}$ has a weak dependence on temperature and the superheat for our casting procedure is modest (nozzle temperature of $\sim 700^{\circ} \mathrm{C}$ and melting temperature above $600^{\circ} \mathrm{C}$ ). Hence, compositional variation was explored. The surface tension of $\mathrm{Al}$ alloyed with $\mathrm{Si}$ does not vary significantly (A.M.Korol'kov, 1956). But by adding small amounts of bismuth (Bi), $\sigma$ can be reduced by up to $50 \%$ (A.M.Korol'kov, 1956). An alloy of composition Al-7wt\%Si-4wt\%Bi $( \pm 0.3 w t \%)$ was prepared from Al-Bi and Al-Si master alloys. Several published works indicate that the surface tension of this alloy, at a temperature of approximately $700^{\circ} \mathrm{C}$ is $0.48 \pm 0.02 \mathrm{Nm}^{-1}$, reduced from a value of $0.85 \pm 0.02 \mathrm{Nm}^{-1}$ for the regular Al-7wt\%Si alloy (A.M.Korol'kov, 1956; Goumiri, Joud, P.Desre \& Hicter, 1979). This lower surface tension alloy was cast twice at different $U$ and these data fall nicely on the correlation in Fig. 3.9, indicating that the wavelength of the defect adjusts to compensate for the changing surface tension. In summary, 
the correlation has been tested by varying $G, U$ and $\sigma$, three of the four variables that enter the abscissa of Fig. 3.9 (varying $\rho$ is not feasible). This directly confirms the capillary nature of the vibrations.

\subsection{Capillary vibrations of a fluid confined in a narrow channel* $^{*}$}

A model problem is considered, consisting of the capillary vibrations of a fluid confined by a channel on three side and with a deformable meniscus being the remaining boundary, as shown in Figure 3.10. Here $\Omega$ is the fluid domain, $\Sigma$ is the solid/liquid boundary and $\Gamma$ is the fluid/air interface. This problem is similar to that of standing gravity waves given in (Lamb, 1932), except that the gravity term is replaced by capillarity which modifies the pressure at the boundary based on the interface curvature. This geometry is similar to that of the PFMS puddle, but without the translating substrate. We are interested in the frequency of standing waves on the meniscus, which depend upon the conditions imposed at the boundaries. Myshkis et al., 1987 solves this problem numerically for an arbitrary contact angle. In general, it is difficult to obtain analytical results for the vibration frequency unless the fluid meets the solid at a $90^{\circ}$ contact angle and the fluid can move freely along the substrate. This will of course be an idealization of the physics of contact line wetting behavior, but is useful as a base model. We can compare these results with the experimental observations of the PFMS puddle.

\footnotetext{
*Section 3.7 has been added to the original manuscript.
} 




Figure 3.10: Geometry of model problem of capillary vibrations in a 2D channel.

\subsubsection{Model problem}

The fluid is treated as inviscid and irrotational, allowing for potential flow theory to be applied. The interface position is given by $x=\eta(y, t)$. It is assumed that the meniscus is normal to the channel walls and can move freely. The governing equations for this problem are

$$
\begin{gathered}
\frac{\partial^{2} \phi}{\partial x^{2}}+\frac{\partial^{2} \phi}{\partial y^{2}}=0, \quad \text { in } \Omega, \\
\rho \frac{\partial \phi}{\partial t}+\frac{1}{2} \rho \nabla \phi \cdot \nabla \phi+P=C(t), \quad \text { in } \quad \Omega, \\
v_{x}=\frac{\partial \phi}{\partial x}, v_{y}=\frac{\partial \phi}{\partial y}, \\
\frac{\partial \phi}{\partial y}=0, \quad \text { at } \quad y= \pm \frac{G}{2}, \\
\frac{\partial \phi}{\partial x}=0, \quad \text { at } \quad x=-h, \\
v_{x}=\frac{\partial \eta}{\partial t}+v_{y} \frac{\partial \eta}{\partial y}, \quad x=\eta(y, t), \\
\text { at } \quad x=1
\end{gathered}
$$




$$
P-P_{a}=\sigma \frac{\partial^{2} \eta}{\partial y^{2}}\left[1+\left(\frac{\partial \eta}{\partial y}\right)^{2}\right]^{-3 / 2}, \quad \text { at } \quad x=\eta(y, t) .
$$

Here, $\phi$ is the velocity potential, $\rho$ is the fluid density, $\sigma$ is the surface tension, $P$ is the pressure in the fluid and $P_{a}$ is the ambient pressure. The approach we will use is to linearize around the base solution of a flat interface with no flow in the fluid, then solve the system with an interface perturbation applied. That is, the base state is given by $\phi=0, \eta=0$ and $P=P_{a}$. The system is linearized by introducing the perturbed parameters $\phi \sim \phi^{\prime}(x, y, t), \eta \sim \eta^{\prime}(y, t)$ and $P \sim P_{a}+P^{\prime}(x, y, t)$. The resulting equations are

$$
\begin{gathered}
\frac{\partial^{2} \phi^{\prime}}{\partial x^{2}}+\frac{\partial^{2} \phi^{\prime}}{\partial y^{2}}=0, \\
\rho \frac{\partial \phi^{\prime}}{\partial t}+P^{\prime}=0, \quad \text { in } \quad \Omega, \\
v_{x}^{\prime}=\frac{\partial \phi^{\prime}}{\partial x}, v_{y}^{\prime}=\frac{\partial \phi^{\prime}}{\partial y}, \\
\frac{\partial \phi^{\prime}}{\partial y}=0, \quad \text { at } \quad y= \pm \frac{G}{2}, \\
\frac{\partial \phi^{\prime}}{\partial x}=0, \quad \text { at } \quad x=-h, \\
P^{\prime}=\sigma \frac{\partial^{2} \eta^{\prime}}{\partial y^{2}}, \quad \text { at } \quad x=\eta^{\prime}(y, t) . \\
v_{x}^{\prime}=\frac{\partial \eta^{\prime}}{\partial t}, \quad x=\eta^{\prime}(y, t),
\end{gathered}
$$


We can nondimensionalize the system using $G$ as the characteristic length scale and $\tau=\left(\rho G^{3} / \sigma\right)^{1 / 2}$ as the characteristic time scale. Using Equation 3.13 at the interface (and differentiating the BC's) we arrive at

$$
\begin{gathered}
\frac{\partial^{2} \bar{\phi}^{\prime}}{\partial \bar{x}^{2}}+\frac{\partial^{2} \bar{\phi}^{\prime}}{\partial \bar{y}^{2}}=0, \quad \text { in } \quad \Omega, \\
\frac{\partial \bar{\phi}^{\prime}}{\partial \bar{x}}=0, \quad \text { at } \quad \bar{x}=-\bar{h}, \\
\frac{\partial^{2} \bar{\phi}^{\prime}}{\partial \bar{t}^{2}}=\frac{\partial^{3} \bar{\phi}}{\partial \bar{x} \partial \bar{y}^{2}}, \quad \text { at } \quad x=\eta^{\prime}(y, t) . \\
\frac{\partial \bar{\phi}^{\prime}}{\partial \bar{y}}=0, \quad \text { at } \quad \bar{y}= \pm \frac{1}{2},
\end{gathered}
$$

Now we can assume the form for $\bar{\phi}^{\prime}$ using separation of variables, as

$$
\bar{\phi}^{\prime}=\cos [n \pi(\bar{y}-1 / 2)] F(\bar{x}) e^{i \omega \bar{t}} .
$$

This form satisfies the boundary conditions of Equation 3.22. Substituting Equation 3.23 into Equations 3.19 - 3.21, we are left with an eigenvalue problem

$$
\frac{d^{2} F(x)}{d x^{2}}-(n \pi)^{2} F(x)=0
$$

with boundary conditions

$$
\begin{gathered}
\frac{d F(\bar{x})}{d \bar{x}}=0, \quad \text { at } \quad \bar{x}=-\bar{h} \\
(n \pi)^{2} \frac{d F(\bar{x})}{d \bar{x}}-(\omega)^{2} F(\bar{x})=0, \quad \text { at } \quad \bar{x}=\eta^{\prime}
\end{gathered}
$$


The result gives the condition for the $\omega$ as

$$
\omega=(n \pi)^{3 / 2} \tanh (n \pi \bar{h})^{1 / 2} .
$$

The function tanh asymptotes to 1 for any argument greater than $\sim 2$. That is, the vibration frequency becomes independent of the channel depth for large depths. We can redimensionalize Equation 3.27 and convert to linear frequency to compare directly with the capillary vibrations observed in the PFMS process. The resulting expression is

$$
f=\frac{(n \pi)^{3 / 2}}{2 \pi} \tau^{-1}
$$

This frequency can be compared directly with that given by Equation 3.3, which gave $f_{\lambda}=1.94 \tau^{-1}$. The $n=1$ mode of vibration corresponds to an interface deviation which is antisymmetric about the plane $y=0$, and gives $f \sim 0.88 \tau^{-1}$. The $n=2$ mode of vibration is symmetric about the plane $y=0$, and gives $f \sim 2.5 \tau^{-1}$. This second mode compares favorably with the experimental results, both in shape deviation and the frequency.

Now that the vibrations in the narrow channel are better understood, a number of issues arise. The model problem lacks many features of the PFMS puddle, such as a base flow in the puddle, solidification, and a dynamic contact angle (among many others). Also, the lowest mode of vibration predicted by the model problem is not the mode observed in the meniscus vibration. The observed vibrations are symmetric about the midplane. We consider modifying the model problem by i) allowing for a curved interface as the base state and ii) applying a wetting condition at the channel boundaries. By bringing in these additional constraints, one can 
distinguish how the the natural frequency of vibration is altered. We will focus on two aspects of the base model and treat them separately.

\subsubsection{Effect of the static contact angle}

Clearly the USM of the PFMS puddle is not flat, the contact angle is typically between $120-150^{\circ}$. The assumption that the flat interface is the appropriate base state is only accurate when the contact angle is $90^{\circ}$. By introducing a curved interface we can analyze the effect of the contact angle on the vibration frequency. However, this significantly complicates the problem and makes obtaining analytic solutions difficult.

When the meniscus is not flat, the curvature of the meniscus is balanced by a pressure jump across the interface. The governing equations remain unchanged, but the interface condition is modified. The equilibrium interface is governed by

$$
P-P_{a}=\sigma \frac{\partial^{2} \eta}{\partial y^{2}}\left[1+\left(\frac{\partial \eta}{\partial y}\right)^{2}\right]^{-3 / 2}, \quad \text { at } \quad x=\eta(y, 0) .
$$

This equation needs to be solved with a specified contact angle at the wall and

the condition that the $\frac{\partial \eta}{\partial y}=0$ at $y=0$. The interface perturbation must now take the form

$$
\eta(y, t)=\bar{\eta}(y, 0)+\eta^{\prime}(y, t)
$$

where $\bar{\eta}(y, 0)$ is the equilibrium interface shape. The kinematic condition at the interface becomes

$$
\frac{\partial \eta}{\partial t}=\frac{\partial \phi}{\partial n}
$$


where $n$ is the outward unit normal to the interface. This problem has been treated numerically for arbitrary contact angles (Myshkis et al., 1987; Kidambi \& Shankar, 2004). The basic approach is to define a deviation function of the form $s(y, t)=\eta(y, t)-\bar{\eta}(y, 0)$, then take a series representation for $s$ and $\phi$ as

$$
\begin{gathered}
s(y, t)=\sum_{n=1}^{\infty} a_{n}(t) f_{n}(y), \\
\phi(x, y, t)=\sum_{n=1}^{\infty} b_{n}(t) g_{n}(y) h_{n}(x) .
\end{gathered}
$$

Numerical techniques are applied to determine the eigenfrequencies. Myshkis et al., 1987, report that $f_{n=2}=2.5 \tau^{-1}$ for a $90^{\circ}$ contact angle (which corresponds to the results of the model problem in Section 3.7.1) and that constant decreases to $\sim 2.1$ as the contact angle is increased to $150^{\circ}$. This result appear to agree more closely with the experimental constant of 1.94. Again, this is the lowest mode which has a symmetry about the midplane. The $n=1$ corresponds to antisymmetric vibrations and occurs at a lower frequency. Kidambi \& Shankar, 2004, present eigenfrequencies only for contact angles between $0-90^{\circ}$, so a direct comparison is not possible.

\subsubsection{Effect of the wetting condition}

The second aspect the we would like to bring into the model is the motion of the contact line. The base model assumes that the contact line can move freely along the interface. The dynamic behavior of contact line motion has been the focus of much study. The dynamic contact angle is a function of the contact line speed and there is typically a hysteresis between the critical advancing and receding angles where the static contact angle can take on range of values (Dussan V, 1979). 
Benjamin \& Scott, 1979, consider gravity-capillary waves under the condition that the contact line is pinned at the container walls, either by a large hysteresis in the contact angle or a natural pinning point such as an brimful container. The result being that pinning the contact line increases the vibration frequency. A modified wetting condition was proposed by Hocking, 1987, where the contact line speed varies linearly with the contact line motion, which is given as

$$
\frac{\partial \eta}{\partial t}=\gamma \frac{\partial \eta}{\partial n},
$$

where $n$ is the unit normal to the solid boundary and $\gamma$ is a constant which gives a measure of the the speed of the contact line as the contact angle varies. This is a useful approximation of the wetting line behavior since there is not a universally accepted model for contact line motion (Billingham, 2002). The limiting cases of the model occur for $\gamma=\infty$, where the wetting line moves freely along the substrate and $\gamma=0$, where the contact line is pinned. The wetting condition ultimately leads to a numerical procedure for computing the eigenfrequencies. Hocking considers the first mode of vibration (antisymmetric about the midplane) in his analysis. The motion of the contact line results in a lowering of the natural vibration frequency.

\subsection{Defect formation mechanism*}

Air entrainment at the upstream meniscus is a potential candidate for the crossstream oscillation defect observed in our casting. The wheel side of the ribbon shows small dimples or cavities along the defect. Air entrainment could result in a small break in heat transfer and lead to the observed defect.

Can a small break in the heat transfer between the molten metal and the wheel

*Section 3.8 has been added to the original manuscript. 


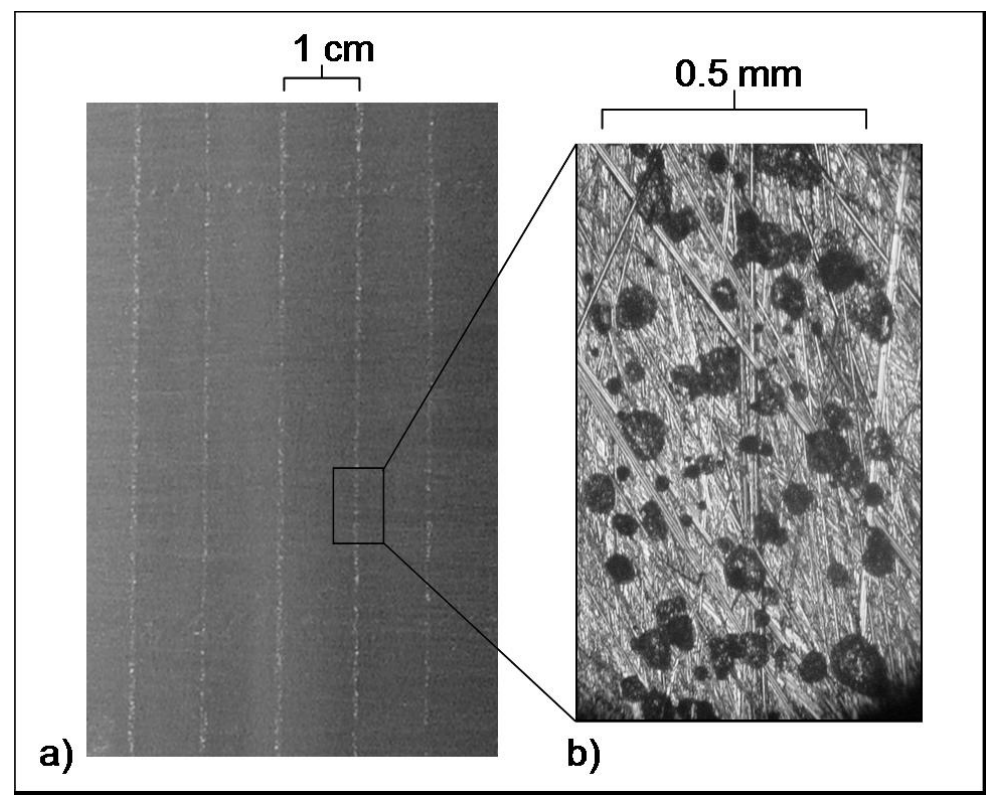

Figure 3.11: BN spray applied to wheel in a straight pattern. The cast direction is from left to right.

result in the cross-stream defect which we observe? To test this idea small strips of boron nitride $(\mathrm{BN})$ aerosol spray are applied to the wheel. $\mathrm{BN}$ is an insulting material which is meant to provoke a response similar to a small air bubble. Figure 3.11 (a) shows BN strips applied to the wheel at a wavelength of $1.0 \mathrm{~cm}$.

It is difficult to accurately characterize the BN strips since it is applied from an aerosol spray bottle. However, the BN spray is applied to a sample piece of copper, sanded with the same 150 grit paper that is used on the wheel before each cast. Figure 3.11 (a) shows an optical microscope image of the copper sample. The BN is not a continuous strip, but rather a scatter of small droplets coating the copper.

When observing the cast ribbon, the marks left by the BN strips are similar to the cross-stream oscillation defect. Figure 3.12 shows the wheel and air sides of 
the ribbon where the straight $\mathrm{BN}$ strips were applied. The defect is registered on both sides of the foil and appears to result in a similar local reduction in thickness.

It is seen in the cast ribbon that the BN strips seem to have little influence on the cross-stream oscillation marks. That is, the cross-stream oscillation pattern passes through the BN lines.

In summary, on the macroscopic level the marks left by the BN strips are difficult to distinguish from the cross-stream oscillation defect. We know that, on a microscopic level, the cross-stream oscillation defect corresponds to a large trench on the free-side with a number of small cavitation along the wheel-side of the ribbon. A similar observation is seen in the profiles of the BN patterns. Figure 3.13 shows a comparison of the profiles. The surface of the ribbon between the defects is relatively flat and uniform. Figure 3.14 shows a comparison of the craters.

\subsection{Thresholds for cross-wave appearance}

The cross-stream wave, while persistent under most processing conditions, does not appear when casting (i) below certain values of $U$ or $G$, (ii) when casting nominally pure $\mathrm{Al}$ or (iii) when the puddle is overly constrained. The cross-wave defect is observed on casting wheels of $3 m$ and $1.9 m$ diameter, with no distinguishable differences in the cross-wave wavelength or onset conditions.

As noted in Section 3.4, the cross-stream wave is not observed for low gap settings. That is, for a fixed $U$, reducing the gap setting will eliminate the crossstream wave. This critical gap was found to be approximately $0.6 \mathrm{~mm}$ for $U=8.8$ $m s^{-1}$. Similarly for a fixed $G$, reducing $U$ will also suppress the wave. For example with $G=1.0 \mathrm{~mm}$, the critical value of $U$ is approximately $5 \mathrm{~ms}^{-1}$. 


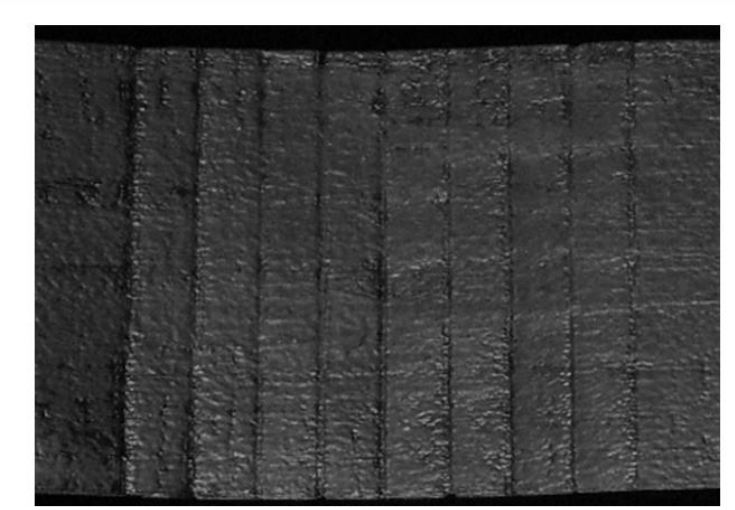

a) Air side

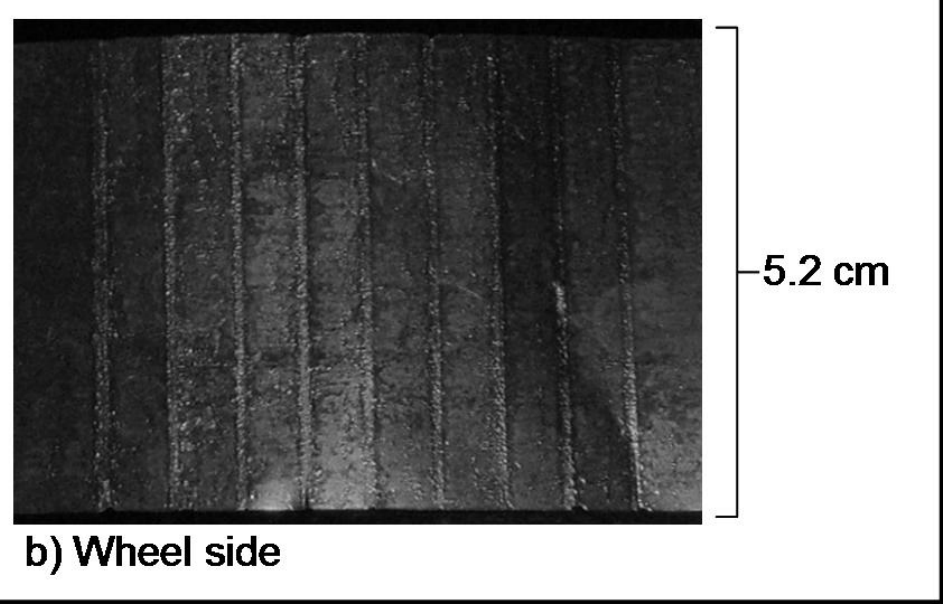

Figure 3.12: Image of ribbon with visible marks from the straight BN strips on both the wheel and air side of the sample. The cast direction is from left to right. 

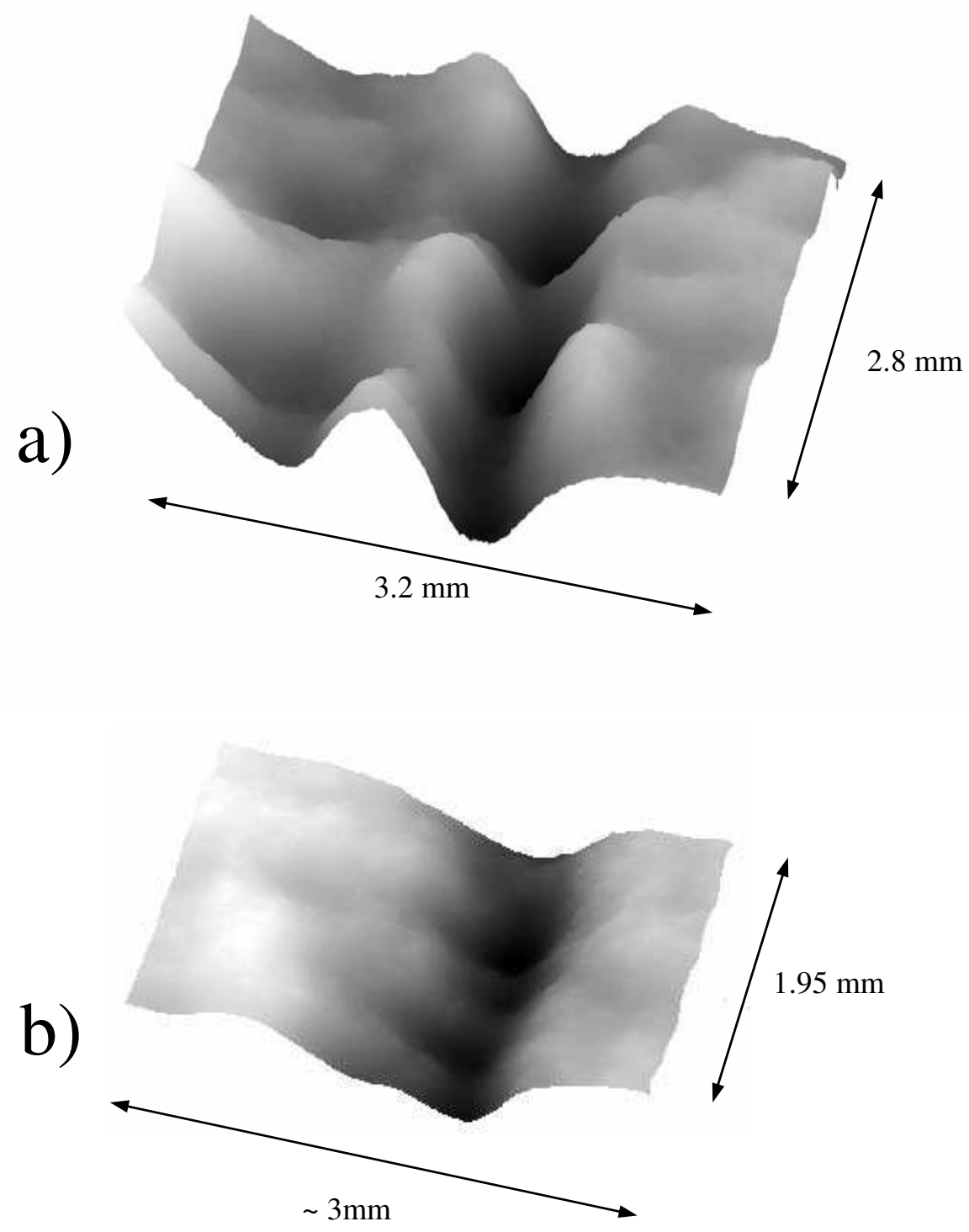

Figure 3.13: Profile of the ribbon surfaces. (a) Scan of the free-side showing the cross-wave defect. The depth of the trench is 90 microns. (b) Scan of free-side showing the effect of the BN droplets. The depth of the trench is 110 microns. The trenches are extending into the solid product. Notice that vertical lengths have been expanded for clarity. 


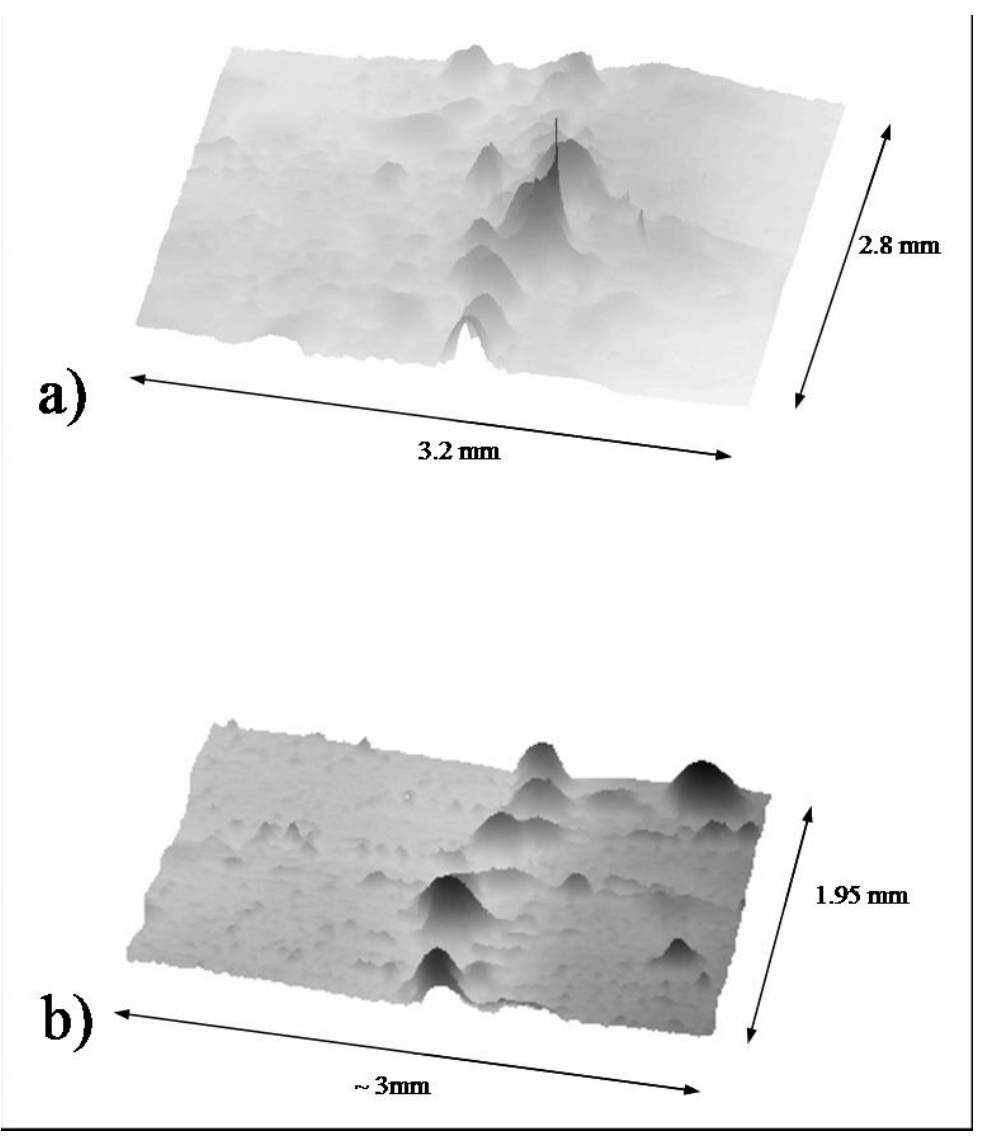

Figure 3.14: Profile of the ribbon surfaces. (a) Scan of the substrate-side showing the cross-wave defect. The size of the craters are approximately 30 microns. (b) Scan of substrate-side showing the effect of the BN droplets. The size of the craters are approximately 25 microns. The craters are extending into the solid product. Notice that vertical lengths have been expanded for clarity. 
The cross-stream wave can also be eliminated by casting nominally pure Al. For Al-Si alloys, more than approximately $2 \% \mathrm{Si}$ is needed for the wave to appear. However, for percentages of $\mathrm{Si}$ in the melt above $2 \%$, the defect wavelength is independent of the alloy composition (Kahn, 2000). That is, $\lambda$ for Al-3\%Si is the same as that for Al-9\% Si. Nominally pure $\mathrm{Al}$ casts $(<2 \% \mathrm{Si})$ do not show a crossstream wave, but rather quite often contain a shorter wavelength $(\approx 1 \mathrm{~mm})$ defect known as the herringbone pattern. This herringbone pattern is a series of fine lines on both sides of the ribbon and has been reported elsewhere (Praisner, Chen \& Tseng, 1995; Carpenter \& Steen, 1992). The Al-Si casts are mostly 7\% Si in Al, but the wave is also present in a range of other alloys that we have cast. These include Al-7\% Si-4\%Bi, as well as the A356 and B356 alloys.

Geometric differences in the puddle may also result in ribbons that do not show the cross-stream wave. It has been found that the wavelength of the defect has no dependence on the overpressure $\Delta P$ when all other parameters are fixed. However, when $\Delta P$ is so low that the puddle takes the constrained configuration of Fig. 3.15(a), the defect is not observed. The herringbone pattern is dominant in this product. During casts where the downstream detachment point is pinned at the edge of the nozzle as shown in Fig. 3.15(b) and the DSM tends to bulge out, the cross-stream wave is also not observed and the herringbone pattern can appear. Nominally pure Al casts almost always take this configuration and Al-Si casts can be forced to do so. Typically the contact-lines in regular Al-Si casts are free to move and take up positions midway between the slot and the nozzle edge, as illustrated in Fig. 3.3. This additional degree of freedom for motion of the puddle may be significant in terms of the modes at which the puddle can vibrate. 


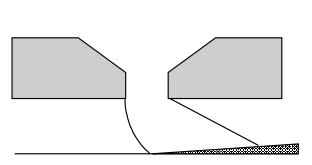

(a)

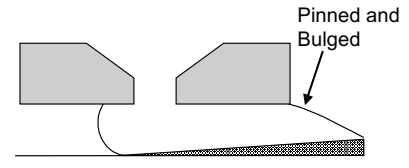

(b)

Figure 3.15: (a) Puddle position during low overpressure casts. (b) Puddle positions during nominally pure $\mathrm{Al}$ casts.

\subsection{Discussion}

In this section, we shall first re-affirm the appropriateness of the scaling found in Section 3.6. We shall then briefly compare our measured frequencies with predictions of two model problems of inviscid capillary vibration, the spherical interface and slot meniscus. Our results will then be contrasted to related observations from the literature. Finally, we offer some remarks on how the puddle oscillation may freeze into the cross-wave shown in Fig. 3.1, the question of mechanism.

The molten metal puddle oscillates at two distinct frequencies. The first $(\approx$ $10 \mathrm{~Hz}$ ) corresponds to a large motion of the puddle forced by a periodic variation of the gap height, whose source is the topography of the moving substrate. The second is a vibration $(\approx 1000 \mathrm{~Hz})$ that corresponds to the natural frequency of liquid inertia countered by surface tension. The source of this puddle vibration is fixed in the lab frame. Since the cross-stream wave in the cast product correlates with the higher frequency, our focus is there.

The surface tension force $\sigma W$ acting per unit width $W$ of the puddle is $\sigma$. To estimate the rate of change of momentum per unit width (mass/length $\mathrm{x}$ velocity/time), one needs an estimate of the volume participating in the oscillation (from which the length scale for the velocity follows, or vice versa). Volume could be $G L W$ or $G^{2} W$ depending on the motion. Observation (e.g. Fig. 3.4) suggests that 
the velocity is $G / \tau$ and hence that the appropriate volume is $G^{2} W$. This is similar to the slot meniscus model discussed below for which the liquid has volume $G L W$ but a volume within a distance $G$ of the meniscus essentially participates in the vibration (volume $G^{2} W$ ). The appropriate balance between inertia and capillarity is then $\sigma \sim \rho G^{3} / \tau^{2}$ from which emerges the capillary time scale $\tau \sim\left(\rho G^{3} / \sigma\right)^{1 / 2}$. Finally, that the coefficient-of-proportionality $C$ in the correlation 3.3 turns out to be order one suggests that the chosen scaling is indeed appropriate.

It is instructive to compare with the classical problem of a liquid sphere of diameter $G$ and density $\rho$, held by capillarity (tension $\sigma$ ), and surrounded by a passive gas (removed from gravity) (Lamb, 1932). If the sphere is slightly deformed and then released, it oscillates with distinct modes shapes at corresponding frequencies. The eigenfrequencies are,

$$
\nu=C_{\nu}\left(\frac{\sigma}{\rho G^{3}}\right)^{1 / 2}
$$

where the constant $C_{\nu}$ depends on the mode number and $C_{\nu} \approx 1.27$ for the lowest harmonic. Compare to $C \approx 1.9$, found in correlation (3.3). Furthermore, the corresponding modal deformation from oblate to prolate spheroid and back again is shared by the shape-change seen in Fig. 3.4.

A meniscus holding an inviscid liquid in between two parallel plates, separated by a distance $G$ and closed on one end, also oscillates with a frequency given by eqn. 3.35. This configuration more closely approximates the constrained geometry of the puddle in PFMS. In this case, in addition to the mode number the constant $C_{\nu}$ depends on the volume of liquid and the angle at which it meets the plates. Myshkis et al., 1987, has solved this problem for the case of fixed contact-angle boundary conditions (moving contact-line). For a contact angle of about $140^{\circ}$ (estimated from Fig.3.4) and the meniscus far from the closed-end, the Myshkis 
analysis predicts $C_{\nu} \approx 0.9$ for the lowest harmonic vibration and $C_{\nu} \approx 2.2$ for next harmonic. The motion of the latter mode is symmetric about the mid-plane and compares favorably to the shape change seen in Fig. 3.4.

An early study of the free-jet melt-spinning process, a process whose puddle is not constrained by a nozzle $(G \rightarrow \infty)$, attributes periodic variations in the ribbon width to capillary oscillations of the puddle, invoking the inviscid sphere capillary oscillations as a model (Anthony \& Cline, 1979). A more recent study of free-jet spinning makes similar observations about ribbon width variations, relating these oscillations to a transition from amorphous to crystalline microstructures (Napolitano \& Meco, 2004). In neither case are cross-stream waves reported. It may be that oscillation of the less constrained free-jet puddle leads to width variations rather than the thickness variations seen in the planar-flow process. In any case, both reports argue the importance of capillary puddle oscillations.

Direct observation of the actual formation mechanism of the craters and troughs of Fig. 3.1 is not possible. Nevertheless, we speculate that deformation due to the oscillation may allow air to be captured at the USM, probably as a thin film which subsequently collapses and leaves the craters seen on the wheel side. The trough on the air-side would then arise as a consequence of the reduced heat transfer, which depends on the mode of film collapse, the net effect being slower solidification over the captured insulating air and, hence, thinner ribbon. This mechanism, although not yet experimentally confirmed, is similar to what has been proposed elsewhere (Huang \& Fiedler, 1981; Anthony \& Cline, 1979). Also, in coating flows, air films between a coating liquid and a substrate are known to be unstable and break into irregular sized bubbles (Kistler, 1993), in much the same manner that we envision maybe happening in this casting flow. 
The evidence presented in this paper links the cross-wave to a capillary oscillation of the puddle. As a natural oscillation, it can be said to be the source of the product defect. The energy for the oscillation can come from any disturbances in the system but the most likely origin is the moving substrate. The puddle filters input frequencies and outputs its natural frequency. Natural oscillations of the puddle cause the cross-wave.

\subsection{Conclusion}

Thickness variations on three disparate length scales, observed in aluminum-alloy ribbon solidified by the PFMS process, are related to three different sources of time-dependence. The slowest time-variation is a gradual change that occurs over the duration of the cast $(3 \mathrm{~s})$ while the other two time-variations are time-periodic (10 Hz and $1000 \mathrm{~Hz})$. Length-scales naturally correspond to these time scales through the wheel-speed $U$. The two slowest thickness variations relate to details particular to our casting machine (wheel shape and heat-up) while the third is largely apparatus-independent (capillary/inertial vibration). This apparatusindependence justifies our emphasis on the cross-wave.

The key difference between the spatially periodic variations is the dependence of wavelength on wheel-speed. Two classes of transfer mechanism, template-transfer and pulse-transfer, are introduced to distinguish features whose wavelengths are independent of speed (template-transfer) and those whose are proportional to speed (pulse-transfer).

On the longest length scale, on order 30 meters, the gradual thinning of ribbon from beginning to end is caused by the gradual shrinking of the gap (on-average) which offers increased hydrodynamic resistance to the flow and, hence, results in 
lower flow rates. This effect is fully understood and can be compensated for by a pre-programmed increasing head of pressure.

On the middle length scale, the circumference of the substrate wheel (about 3 meters), the spatially periodic thinning and thickening of the ribbon is caused by the hills and valleys of the wheel as they pass underneath the nozzle. The puddle moves with a time-periodic component corresponding precisely to the spatial wavelength of these spatial variations. This feature is thus well-understood as the forcing of the gap $G(t)$ at a frequency of about $10 \mathrm{~Hz}$. The wavelength of this feature is independent of the wheel speed and, hence, represents a template-transfer mechanism.

On the smallest scale of interest in this paper, one observes dramatic thickness variations with wavelengths on order one centimeter. These cross waves are often viewed as defects. This local thinning shows up as a line of craters on the wheel-side with a corresponding trough on the air-side. The wavelength is found to depend linearly on speed and, hence, corresponds to a pulse-transfer mechanism. Furthermore, the wavelengths are identified with a spectral peak in puddle vibrations on the order of $1000 \mathrm{~Hz}$. A correlation of the feature with the inviscid capillary oscillations of the puddle demonstrates that it is a natural frequency of the puddle, akin to the oscillations of an inviscid sphere or the vibrations of a meniscus in a slot.

The cross-wave defect is not observed under all conditions. Casting at low $G$ and/or low $U$ and, in Al-Si casts, at low Si content suppress the cross wave. With the exception of the effect of $U$, common among these influences is the degree to which the the puddle is constrained. A sufficiently constrained puddle will not 
oscillate. On the other hand, the influence of $U$ may have more to do with the excitation of the puddle. Sufficient excitation is needed for oscillations.

\subsection{Acknowledgements}

Experiments were supported by NSF grants DMI-0124730 and DMI-0423791. The authors would like to thank Dr. M.Vogel for help with image analysis and P.Wiechecki for help with the experiments. Also we would like to thank Dr. Steve Weinstein (Eastman Kodak) for many useful discussions. We thank ALCOA and KBAlloys Inc. for supplying materials for these experiments.

\subsection{Appendix}

\subsubsection{Time dependent behavior of gap height}

Because of the large radius of our substrate $\left(R_{w}=0.45 m\right)$, the thermal expansion of the wheel for even modest temperature increase $\left(\Delta T_{w}>50^{\circ} \mathrm{C}\right.$ for $1 \mathrm{~kg}$ of aluminum) is quite significant (of order $0.3 G$ ) when compared to our small gap sizes $(G \approx 1 m m)$.

The most accurate measurement of this shrinking gap uses real-time measurements via high-speed photography of a run-out meter (point-wise data). Such measurement is time-consuming and not practical for every cast. Therefore, an approximate method that accounts for gap-shrinkage (wheel expansion) has been developed and validated against the direct measurements, as described below.

A set of experiments was performed where an average rate of substrate expansion is measured under different casting conditions. This rate of gap-shrinkage $\left(\frac{d G}{d t}\right)_{\text {avg }}$ is then applied to each cast in order to get $G(t)$, given as 


$$
G(t)=G(0)-\left(\frac{d G}{d t}\right)_{a v g} t,
$$

where $G(0)$ is the initial gap setting. This method neglects the out-of-roundness of the substrate, but still gives a good estimate of the gap-shrinkage with time. The calculated values of $G(t)$ is then used to scale the point-wise data.

Using point-wise data (Fig. 3.8) shows the best correlation of the experimental data. These experimental conditions vary to a limited extent only throughout a particular cast, however. In contrast, in Fig. 3.9, $\sigma, U, G$ and $\lambda$ all vary in the raw data that correlates. Fig. 3.9, however, uses Equation (3.36) to calculate a cast-averaged value of $G$. The greater uncertainty due to the approximation seems to be compensated for by averaging over a large number of data (e.g. 200 points for a $30 \mathrm{~m}$ ribbon) with the end result that Figs. 3.8 and 3.9 present correlations of similar quality ( $R^{2}$ factor).

On the other hand, the data that comprise Figs. 3.8 and 3.9 all come from the same laboratory, the same apparatus and, indeed, all were taken by the same team of investigators. Other data taken on a different casting apparatus, with different human operators and using a different technique to set and measure the hot-gap at the start of a cast are available in our archives. We present this data here i) to convey a sense of how prevalent cross-waves are and how they correlate to the capillary oscillations under quite diverse conditions and ii) to illustrate how using Equation (3.36) to scale point-wise data leads large scatter in the data.

Fig. 3.16 shows the results for approximately 10 casts from our archives. Notice that the scatter in the data increases as $G$ becomes smaller (moving to the right on the $\mathrm{x}$-axis). This trend is expected since the wheel out-of-roundness is a more significant variation in $G$ at lower gap heights. The experimental constant $(C \approx 2)$ 


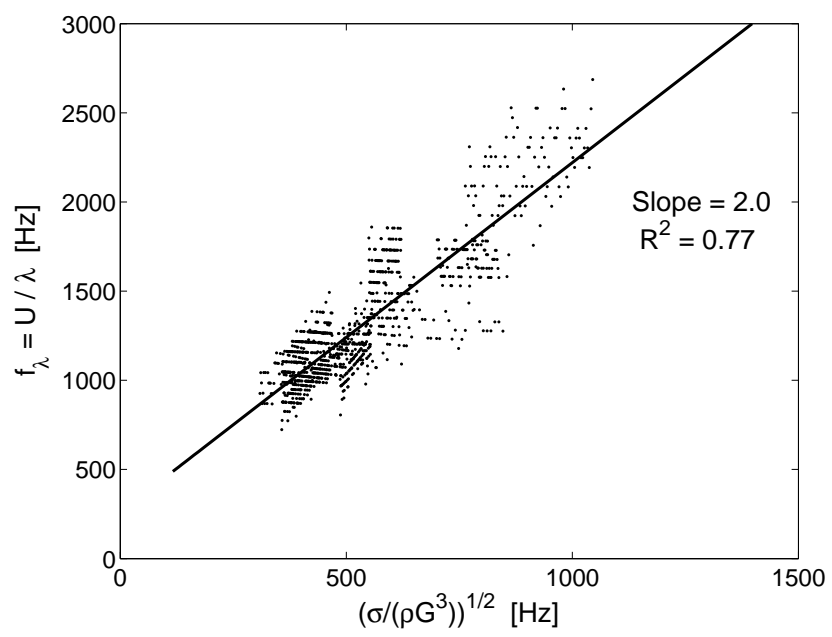

Figure 3.16: Cross-stream defect frequency plotted against capillary frequency, without accounting for substrate out-of-roundness, resulting in large scatter in experimental data.

based on the slope of the data presented here using the calculated $G(t)$ are in reasonable agreement with Fig. 3.8 where $G(t)$ was measured from the run-out meter. As expected, however, the correlation in Fig. 3.16 is not as good $\left(R^{2}\right.$ of 0.77 vs 0.95$)$. 
Table 3.3: Cast-averaged data and control parameters for data appearing in the paper. Cast (1), (2) and (3) correspond to Fig. 3.8. Casts TBFA03-01 through TBSP05-23 appear in Fig. 3.9. The final 10 casts appear in Fig. 3.16

\begin{tabular}{lccccc} 
Cast ID & $T(m m)$ & $U\left(m s^{-1}\right)$ & $\Delta P(P a)$ & $\bar{G}(m m)$ & $\lambda(m m)$ \\
\hline TBSU02_21* & 0.13 & 10.5 & 2900 & 0.93 & 7.2 \\
TBFA03_01 & 0.138 & 8.83 & 2497 & 1.34 & 11.6 \\
TBFA03_02 & 0.150 & 8.77 & 2791 & 1.34 & 11.0 \\
TBFA03_04 & 0.133 & 8.86 & 2587 & 1.33 & 10.3 \\
TBSP03_02 & 0.112 & 8.66 & 3922 & 0.85 & 5.3 \\
TBSP03_04 & 0.122 & 8.92 & 3888 & 0.86 & 6.0 \\
TBSP03_05 & 0.131 & 8.90 & 4097 & 0.87 & 5.7 \\
TBSP03_08 & 0.123 & 8.87 & 4182 & 0.85 & 5.3 \\
TBSP03_26 & 0.173 & 8.81 & 3810 & 1.16 & 10.1 \\
TBSU02_10 & 0.142 & 10.68 & 4375 & 1.04 & 8.8 \\
TBSU03_10 & 0.14 & 8.8 & 4300 & 0.89 & 4.6 \\
TBSU03_16 & 0.156 & 8.77 & 3455 & 1.10 & 7.6 \\
TBSU03_23 & 0.195 & 8.81 & 3579 & 1.16 & 9.4 \\
TBSU03_25(1) & 0.161 & 8.74 & 3290 & 1.16 & 9.4 \\
TBSU03_28(2) & 0.179 & 8.73 & 3689 & 1.15 & 7.9 \\
TBSU04_11 & 0.116 & 11.93 & 4044 & 0.86 & 7.2 \\
TBSU04_14 & 0.243 & 5.94 & 4479 & 0.88 & 4.7 \\
TBSU04_16 & 0.093 & 13.96 & 4763 & 0.77 & 7.7 \\
TBSU04_24 & 0.131 & 11.87 & 4867 & 0.87 & 6.9 \\
TBSU04_25 & 0.159 & 8.80 & 4437 & 0.88 & 5.5 \\
TBSU04_26 & 0.211 & 7.09 & 3977 & 0.89 & 6.0 \\
TBSU04_28 & 0.066 & 11.94 & 2157 & 0.73 & 6.7 \\
TBSU04_31 & 0.126 & 11.90 & 4348 & 0.87 & 6.7 \\
TBFA02_03 & 0.131 & 9.46 & 4278 & 0.82 & 5.9 \\
TBFA02_06 & 0.15 & 9.51 & 4100 & 1.17 & 9.6 \\
TBFA04_13 & 0.185 & 7.10 & 2869 & 1.41 & 8.6 \\
TBFA04_14 & 0.171 & 7.01 & 2723 & 1.41 & 8.3 \\
TBFA04_15 & 0.180 & 7.20 & 3013 & 1.40 & 9.1 \\
TBSP05_05 & 0.121 & 11.92 & 3114 & 1.37 & 14.5 \\
TBSP05_10** & 0.119 & 8.59 & 1639 & 0.75 & 6.8 \\
TBSP05_11** & 0.109 & 8.37 & 1756 & 1.10 & 10.3 \\
TBSP05_12 & 0.119 & 8.59 & 1639 & 0.75 & 6.8 \\
TBSP05_13(3) & 0.16 & 8.5 & 3500 & 0.88 & 6.5 \\
TBSP05_23*** & 0.17 & 11.9 & 2300 & 1.38 & 1.7 \\
MKSP00_23 & 0.219 & 7.55 & 4072 & 1.22 & 7.2 \\
MKSP00_25 & 0.197 & 7.12 & 4078 & 0.96 & 5.2 \\
MKSU00_08 & 0.222 & 8.76 & 3606 & 1.13 & 7.2
\end{tabular}


Table 3.3: (Continued)

\begin{tabular}{lccccc} 
Cast ID & $T(\mathrm{~mm})$ & $U\left(\mathrm{~ms}^{-1}\right)$ & $\Delta P(\mathrm{~Pa})$ & $\bar{G}(\mathrm{~mm})$ & $\lambda(\mathrm{mm})$ \\
\hline BRFA99_18 & 0.136 & 8.40 & 2754 & 0.70 & 3.9 \\
BRSU99_16 & 0.16 & 6.68 & 3800 & 1.1 & 6.2 \\
BRSU99_22 & 0.16 & 7.8 & 4100 & 0.86 & 4.7 \\
BRSU99_08 & 0.11 & 9.4 & 4100 & 1.02 & 5.7 \\
MKSP00_10 & 0.16 & 8.8 & 3700 & 1.3 & 7.5 \\
MKSP00_12 & 0.25 & 8.3 & 7500 & 1.2 & 7.2 \\
MKSP00_21 & 0.2 & 7.8 & 4000 & 1.3 & 8.0 \\
\hline
\end{tabular}

${ }^{*} B=3 \mathrm{~mm}$,

${ }^{* *} \mathrm{Al} \% \mathrm{Si} \mathrm{Si} \% \mathrm{Bi}$

${ }^{* * *} W=25 \mathrm{~mm}$ 


\section{Chapter 4}

\section{Capillary Dynamics of Coupled}

\section{Spherical-cap Droplets*}

\subsection{Overview}

Liquid droplets tend to spherical shapes under the action of surface tension. The vibration of a sphere is a classical example of the competition between liquid inertia and capillarity. As summarized by Lamb (1932), Lord Rayleigh (1879) predicted the frequency of small oscillations (mode $n$ ),

$$
\omega_{n}{ }^{2}=n(n-1)(n+2)\left(\sigma / \rho r^{3}\right),
$$

where $\sigma, \rho$ and $r$ are surface tension, liquid density and radius of the undeformed sphere, respectively (see also Webb (1880)). The frequencies $\omega_{0}=0$ and $\omega_{1}=0$ owing to translational invariance and the constant-volume constraint, respectively. The lowest non-zero frequency $\omega_{2}$ plays an important role in application. The prediction of Rayleigh has been verified in experiment and its relevance welldocumented (e.g. Trinh \& Wang (1982) and Bisch et al. (1982)).

Suppose that the Rayleigh droplet is now constrained by pinning it on a circle of contact (figure 1a,b). How will the constraint influence the frequency of oscillation? Will it go up or down? A reasonable answer might be that the constrained droplet will oscillate faster, just as a shorter violin string has higher pitch. This reasoning and the conclusion would be correct if one restricts to a fixed mode; that is,

*Reprinted with permission from the Journal of Fluid Mechanics, E.A. Theisen, M.J. Vogel, C.A. López, A.H. Hirsa and P.H. Steen, "Capillary dynamics of coupled spherical-cap droplets" 580, p495-505, 2007. Appendix 4.6 has been added to the original manuscript. 

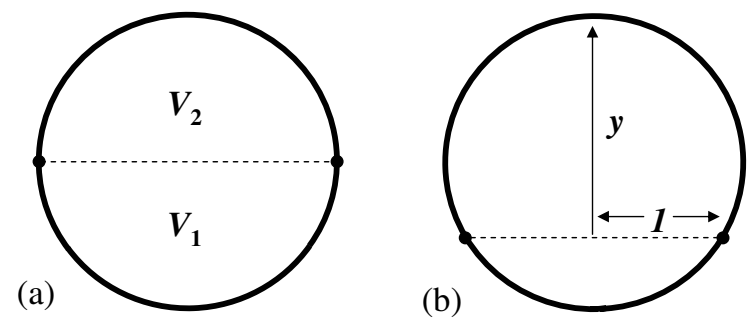

Figure 4.1: Pinning along a circle-of-contact (dotted line) varies in position from a) the equator $\left(V_{1}-V_{2}=0\right)$ to b) the southern hemisphere $\left(V_{1}-V_{2}<0\right)$.

pinning the surface increases the frequency for any particular mode. On the other hand, if all modes are allowed, the situation is not so straightforward. It turns out that pinning can 'activate' the translational mode, raising its frequency from zero and introducing a slow oscillation. The slow frequency $\omega_{2}^{*}$ depends on where the pinning circle is placed (figure 4.1). We report below $(L=0$ limit of $(4.14))$ that $\omega_{2}^{*} / \omega_{2}=\frac{\sqrt{3}}{4}(y-1 / y)$ where $y \geq 1$ is the distance from the north pole to the constraining circle scaled by the radius of the constraint (figure $4.1 \mathrm{~b})$ ). Thus, the slow frequency increases monotonically from zero for a pinned-equator constraint (figure 4.1a))to infinity for a pinned point at the south pole. In particular, the translational-mode frequency crosses the unconstrained slow frequency $\left(\omega_{2}^{*} / \omega_{2}=\right.$ 1) at $y_{c} \equiv \frac{2+\sqrt{7}}{\sqrt{3}} \sim 2.68$. Hence, according to our model, the correct answer depends on where the pinned constraint is placed. The emergence of a linear, low-frequency center-of-mass oscillation in a related problem has been reported by Strani \& Sabetta (1984). The translational mode introduced by the pinned circular-constraint has not been studied before, however, as far as we are aware.

The above geometry-of-constraint is generalized in two ways in our study. First, two sub- or super-hemispherical caps can be joined at the constraining circle giving a non-spherical overall shape. Second, the pinning circle can be 'inflated' to be a 
cylindrical tube of length $2 L$ with spherical caps pinned to each of the tube-ends (figure 4.2). This family of undeformed capillary surfaces is characterized by two control parameters, the sum of spherical-cap volumes $\left(V_{1}+V_{2}\right)$ and the connection half-length $L$. Figure 4.2 shows symmetric and anti-symmetric equilibria (interdroplet pressures equal) distinguished by $V_{1}+V_{2}<(4 / 3) \pi r^{3}$ (figure $4.2 \mathrm{a}$ ) or $V_{1}+$ $V_{2}>(4 / 3) \pi r^{3}$ (figure $\left.4.2 \mathrm{~b}\right)$.

The translational motion of the droplet-droplet system is modeled by restricting to spherical-cap shapes. The benefit of this approximation is that finite-amplitude dynamics become tractable to study. The motions are described by a $2 \mathrm{D}$ system while Rayleigh's infinitesimal motions are described by a partial differential equation. For purposes of this paper, validity of the approximation is tested by comparing with experiment.

Motivation for this study comes from practical application. Two pressurecoupled droplets have a double-welled surface energy landscape. With a mechanism to trigger from one well to another, such as with an electro-osmotic pump placed in the tube (Vogel et al. (2005)), the system becomes active. A number of applications that exploit capillary bi-stability are under development. These include optical micro-lens devices (Lopez et al. (2005)). For these, understanding the dynamics of the droplet-droplet configuration and especially, the lowest frequency mode, is important.

Droplet motions dominated by inertia and surface tension are generally also influenced by liquid viscosity. Even though the Reynolds number is order one hundred, viscous effects are non-negligible according to experiment. The proposed inviscid model of the translational-mode dynamics is readily modified to include viscous damping. 


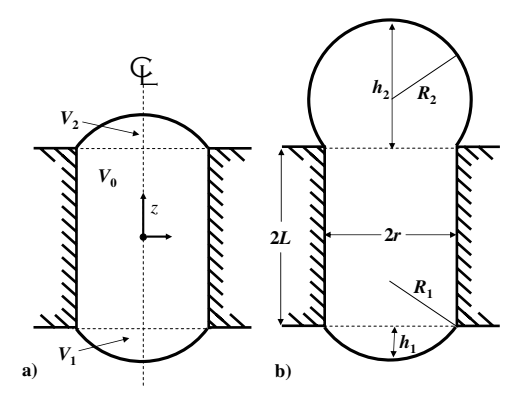

Figure 4.2: Schematic of coupled droplets pinned at tube-ends of radius $r$. $V_{1}$ and $V_{2}$ are protruding volumes. Typical equilibria for a) $V_{1}+V_{2}<(4 / 3) \pi r^{3}$ and b) $V_{1}+V_{2}>(4 / 3) \pi r^{3}$. Equilibrium occurs for menisci with equal curvatures, $R_{1}=R_{2}$.

In section 4.2 we develop a dynamic model that governs the center-of-mass of the double-droplet system. The equations of motion predict how the droplets exchange volume when finite-amplitude disturbances are applied. In section 4.3 these predictions are compared to experimental results. Finally, in section 4.4 we discuss linear and some finite-amplitude predictions of the dynamic model (including viscosity), putting them in the context of other studies.

\subsection{Dynamical model}

Let $z_{c}$ be the center-of-mass for the total liquid volume (figure 4.2), in the tube and both droplets, $V_{T}=V_{0}+V_{1}+V_{2}$. Newton's law takes the form,

$$
\frac{d}{d t}\left(\rho V_{T} \frac{d z_{c}}{d t}\right)=F_{\sigma}+F_{\mu} .
$$

Owing to axisymmetry, the net force $F=F_{\sigma}+F_{\mu}$ acting on the control volume is in the axial direction, $F \mathbf{e}=\int_{\partial V_{T}} \mathbf{T} \mathbf{n} d A$, where $\mathbf{T}=-p \mathbf{1}+2 \mu \mathbf{D}$ is the stress tensor, $\mu$ is the viscosity, $\mathbf{D}$ the symmetric part of the velocity gradient tensor, $\mathbf{e}$ 
the axial unit vector and $\mathbf{n}$, the unit normal. The net force splits into a capillary $F_{\sigma}$ and a viscous part $F_{\mu}$ according to the split between pressure and deviatoric stress using the assumption of a stress-free liquid/gas interface. The pressure contribution, from the spherical-cap droplets of radius $R_{i}$, is evaluated by the Young-Laplace relationship, $p_{i}=2 \sigma / R_{i}$ for $i=1,2$, to yield $F_{\sigma}=\pi r^{2}\left(p_{1}-p_{2}\right)=$ $2 \pi r^{2} \sigma\left(1 / R_{1}-1 / R_{2}\right)$. The viscous part arises due to tractions along the tube wall $\partial V_{0}$ and can be written $F_{\mu}=2 \mu \int_{\partial V_{0}}$ eDn $d A=2 \mu(4 \pi r L / \tau) f$ where $f$ is the dimensionless viscous force and $\tau$ a characteristic time-scale. It is convenient to scale all lengths with $r$, volumes with $(4 / 3) \pi r^{3}$ and to choose a time-scale to balance the inertia and capillary terms, $\tau^{2} \equiv \rho r^{3} / \sigma$. Henceforth, unless otherwise noted, variables will be dimensionless. We will use the same symbol for scaled as for previously unscaled variables. Substitute forces into (4.2), use the scales just defined, and rearrange to find,

$$
\frac{d^{2}}{d t^{2}}\left(V_{T} z_{c}\right)-\frac{3}{2}\left(\frac{1}{R_{1}}-\frac{1}{R_{2}}\right)=6 R e^{-1} L f
$$

where $R e^{-1} \equiv(\mu / \rho)(\rho / r \sigma)^{1 / 2}$ represents the ratio of viscous to inertial forces.

Inviscid behavior dominates the dynamics and we put $R e^{-1}=0$ for now in order to obtain a closed-form equation for the dissipationless motions. Viscous effects are discussed in Section 4.4. The magnitude of the center-of-mass for each droplet $z_{i}$ has a closed-form expression in terms of droplet height $h_{i}$ and length $L, z_{i}=L+(1 / 2) h_{i}\left(2+h_{i}^{2}\right) /\left(3+h_{i}^{2}\right)$. Droplet radii are given by $2 R_{i}=\left(h_{i}+\right.$ $\left.1 / h_{i}\right)$ and volumes by $V_{i}=(1 / 8) h_{i}\left(3+h_{i}^{2}\right)$ (figure 4.2$)$. System center-of-mass is related to component center-of-mass by $z_{c} V_{T}=-z_{1} V_{1}+z_{2} V_{2}$ where the tube volume drops out since its center-of-mass stays fixed at $z=0$. Substituting these relationships into (4.3), two second-order systems, each in terms of dependent variables $h_{1}$ and $h_{2}$, emerge. In view of the constant volume constraint, $h_{1}$ and 
$h_{2}$ are not independent and therefore the more convenient variables are $(\Theta, \lambda) \equiv$ $\left(V_{1}-V_{2}, V_{1}+V_{2}\right)$. In order to express (4.3) in terms of $(\Theta, \lambda)$, mappings between coordinates pairs $\left(h_{1}, h_{2}\right),\left(V_{1}, V_{2}\right)$ and $(\Theta, \lambda)$ are needed. The first mapping is nonlinear (everywhere invertible, though) and the second linear. Indeed, one can build the functions,

$$
h_{1}=H_{1}(\Theta, \lambda) ; \quad h_{2}=H_{2}(\Theta, \lambda)
$$

from the inverse $h_{i}=h_{i}\left(V_{i}\right)$ of the cubic expression above; explicitly, $h=[4 V+$ $\left.\left(1+(4 V)^{2}\right)^{1 / 2}\right]^{1 / 3}+\left[4 V-\left(1+(4 V)^{2}\right)^{1 / 2}\right]^{1 / 3}$. In summary, (4.3) can be rewritten,

$$
\frac{d}{d t}\left(A(\Theta ; \lambda, L) \frac{d \Theta}{d t}\right)+C(\Theta ; \lambda)=0
$$

where

$$
A(\Theta ; \lambda, L) \equiv\left(H_{1}+H_{2}+3 L\right)
$$

and

$$
C(\Theta ; \lambda) \equiv 9\left(\frac{\left(H_{1}-H_{2}\right)\left(1-H_{1} H_{2}\right)}{\left(H_{1}^{2}+1\right)\left(H_{2}^{2}+1\right)}\right) .
$$

The rate of volume redistribution $d \Theta / d t$ is multiplied by $A$ which accounts for the influence of tube length and spherical-cap geometries on the center-of-mass of the system. Note that parameter $L$ appears only in function $A$ and serves to tune the strength of inertia. The function $C$ represents the restoring force corresponding to a single- or double-well potential depending on whether $\lambda<1$ or $\lambda>1$, respectively. 


\subsubsection{Phase-plane solution}

The phase-plane is organized by the equilibria which depend on $\lambda$. Equilibrium solutions of (4.5) are the zeros of $C(\Theta ; \lambda)$ and can be read off as $H_{1}=H_{2}$ and $H_{1} H_{2}=1$. The null solution of symmetric shapes bifurcates into the family of anti-symmetric shapes at $\lambda=\lambda_{h s} \equiv 1$ where shapes are hemispherical(hs),

Branch $1\left(H_{1}=H_{2}\right): \Theta_{1}^{*}(\lambda)=0 ;$ for all $\lambda$.

Branch $2\left(H_{1} H_{2}=1\right) \quad: \quad \Theta_{2}^{*}(\lambda)= \pm\left(\lambda^{2}-\frac{3}{4} \lambda^{2 / 3}-\frac{1}{4}\right)^{1 / 2} ;$ for $\lambda \geq 1$.

The pitchfork bifurcation is plotted in Figure 4.3. (Measured steady shapes compare favorably to the pitchfork prediction (Vogel et al. (2005)). Two representative phase-plane plots show the finite-amplitude behavior. Below $\lambda_{h s}$, a family of limit cycles surrounds the symmetric steady state (null solution), corresponding to finite-amplitude periodic oscillations. Above $\lambda_{h s}$, the null solution becomes a saddle point. Small but finite-amplitude initial displacements from either the upor down- equilibrium-state again result in limit cycle oscillations about that state. However, large disturbances lead to looping solutions where the system volume oscillates between being predominantly above or below the tube mid-plane. The trajectories from the saddle point at $\Theta=0$ define the separatrix, separating initial conditions that lead to looping solutions from those for which $\Theta(t)$ does not change sign. This qualitative behavior can be ascertained using stock phase-plane tools (e.g. Minorsky (1962)). The solutions plotted in figure 4.3 were computed using a standard ODE solver (MATLAB). The phase plane is qualitatively like that of the two-point attractor with quadratic energy wells (e.g. Thomson \& Hunt (1986)).

Linearizing (4.5) about a steady-state in the standard way gives, $A_{*} d^{2} \Theta / d t^{2}+$ 


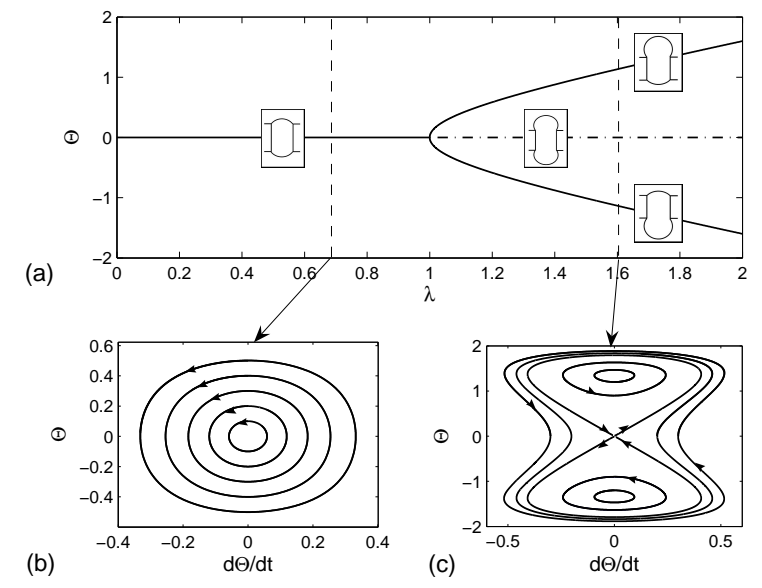

Figure 4.3: (a) Bifurcation diagram with typical equilibrium shapes sketched. Typical phase-plane solution for (b) $\lambda<1(\lambda=0.7)$ and (c) $\lambda>1(\lambda=1.6)$.

$C_{*} \Theta=0$ where $A_{*}$ and $C_{*}$ are coefficients that depend on $\lambda$ through $\Theta_{i}^{*}, i=1,2$. Eigenvalues can be read off as $\pm \sqrt{C_{*} / A_{*}}$. Along the branch 1 and for $\lambda<1$, the eigenvalues are a complex conjugate pair $\pm i \omega_{1}^{*}$ with values (using the notation $\left.y \equiv H_{1}=H_{2}\right)$

$$
\omega_{1}^{* 2}=\frac{24\left(1-y^{2}\right)}{\left(y^{2}+1\right)^{3}(2 y+3 L)} \text { for } y<1 .
$$

For this lens-like family, droplet apex $y$ and total volume $\lambda$ are related by $\lambda=$ $\frac{1}{4} y\left(3+y^{2}\right)$. For $\lambda>1$ the eigenvalues $\pm \gamma_{1}^{*}$ are real with values,

$$
\gamma_{1}^{* 2}=\frac{24\left(y^{2}-1\right)}{\left(y^{2}+1\right)^{3}(2 y+3 L)} \text { for } y>1
$$

This gives the rate of decay from the unstable equilibria and will be relevant for trajectories that just carry the system over the energy barrier, for example. For the family of equilibria along branch 2 (upper), we use the notation $y \equiv H_{2}=H_{1}^{-1}$. 
For these states, droplet height $y$ and total volume $\lambda$ are related by $\lambda=\frac{1}{8}(y+1 / y)^{3}$. The eigenvalues are complex conjugates $\pm i \omega_{2}^{*}$ with values,

$$
\omega_{2}^{* 2}=\frac{12(y-1 / y)^{2}}{(y+1 / y)^{2}(y+1 / y+3 L)} \text { for } y>1 .
$$

For the lower segment of branch 2 , replace $y$ by $1 / y$ in (4.10) and restrict to $y<1$ to find the same eigenvalues, as must be the case. Note that at $\lambda=1(y=1)$ the system has a double-zero eigenvalue. The frequencies $\omega_{1}^{*}($ for $\lambda<1)$ and $\omega_{2}^{*}$ (for $\lambda>1$ ) are plotted in dimensional form as a solid line in figure 4.4. Data are discussed below. Linear frequencies could have alternatively been reported as functions of $\lambda$ rather than $y$.

To summarize the linear behavior, branch 1 is a center below critical and a saddle point above critical while upper and lower segments of branch 2 , equivalent by symmetry, are centers. The transition at $\lambda_{h s}$ does not depend on $L$. However the magnitudes of the eigenvalues do depend on $L$, with large $L$ corresponding to slower vibration frequencies. That is, for large tube volumes, the capillary forces acting at the tube ends have a smaller influence on the center of mass of the system; capillarity takes longer to restore shapes.

Although periods of oscillation for finite-amplitude limit cycles can be obtained computationally from (4.5), an analytic expression is readily derived. To this end, a first-integral of (4.5) can be written,

$$
\frac{1}{2}\left(A(\Theta ; \lambda) \frac{d \Theta}{d t}\right)^{2}+U(\Theta ; \lambda)=\text { const } \equiv E,
$$

where $U(\Theta)$ is obtained by quadrature,

$$
U(\Theta)=\int_{0}^{\Theta} A(s) C(s) d s .
$$


(a)

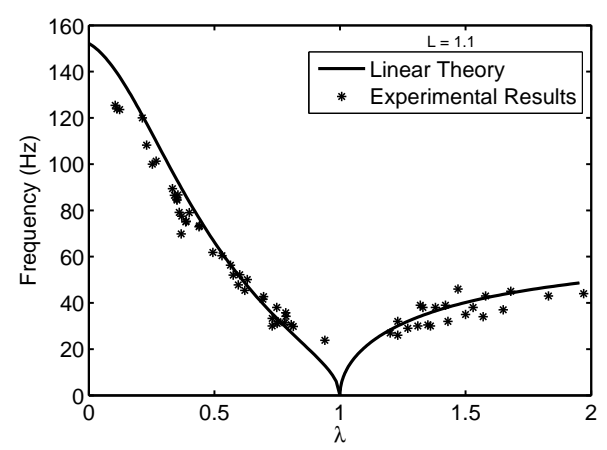

(b)

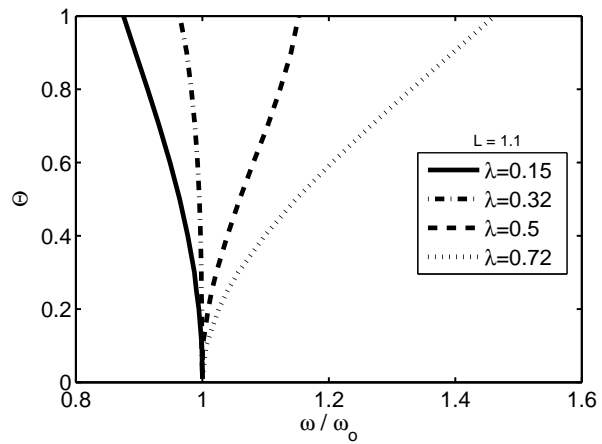

Figure 4.4: (a) Dimensional vibration frequency $\omega_{1}^{*}$ for $\lambda<1$ and $\omega_{2}^{*}$ for $\lambda>1$ according to linear theory (solid line) and compared to experiment (symbols). (b) Frequency of oscillation for limit cycles of amplitude $\Theta$ scaled by the linear frequency $\omega_{0} \equiv \omega_{1}^{*}$.

It can be shown that the product of $A(\Theta ; \lambda) C(\Theta ; \lambda)$ is an odd function of $\Theta$ which leads to a $U(\Theta)$ that is even about $\Theta=0$. Solving (4.11) for $d \Theta / d t$, noting the separability of the variables $\Theta$ and $t$, allows the period of oscillation $T$ for a limit cycle to be expressed in closed form,

$$
T=2 \int_{\Theta_{+}}^{\Theta_{-}} \sqrt{\frac{A}{2(E-U)}} d \Theta .
$$

Here $\Theta_{-}$and $\Theta_{+}$are the minimum and maximum of $\Theta$ along the closed trajectory in the phase plane, respectively.

For oscillations symmetric about $\Theta=0$ (branch 1 with $\lambda<1$ and the looping solutions), $\Theta_{-}=-\Theta_{+}$and expression (4.13) can be evaluated as the amplitude $\Theta \equiv \Theta_{+}$of the disturbance is increased. Figure 4.4(b) shows how the frequency changes with amplitude for sub-hemispherical droplets. For finite-amplitude oscillations, the restoring capillary force varies from soft to hard as $\lambda$ is increased from 0 to 1 . These results are consistent with those from a multiple time scales pertur- 
bation approach (Lindstedt's method). Among other finite-amplitude information that can be obtained from eqns (4.11) and (4.12) is an estimate of the minimum amplitude of disturbance needed to blow the liquid totally out of the tube (future work).

\subsection{Experimental results}

The experimental setup consists of a Teflon plate $(1.82 \mathrm{~mm}$ thick) with a small circular hole (0.83 $\mathrm{mm}$ radius) bored through, as depicted in figure 4.5a. A micro-syringe is used to feed water to control the volume of the droplets. Pinned contact-lines are well maintained as verified through image-analysis. The plate separates two pressure-controlled chambers. Gravity is weak relative to surface tension (Bond number $B \equiv \rho g r^{2} / \sigma \sim 0.1$ ) yet gravity has a non-negligible effect. It adds a hydrostatic head to the bottom droplet to make its mean pressure greater and along the length of both droplets it distorts the droplet shape from spherical. To counter the head, a pressure bias in the lower chamber is applied. Further details of the experimental setup are given elsewhere (Hirsa et al. (2005)).

We begin by fixing the total volume of the droplet then slightly adjusting the pressure bias to achieve an equilibrium state of zero volume-difference. A microstepper motor is then used to apply a pressure pulse in one air chamber to perturb the droplet. The duration of the pressure pulse is less than $100 \mathrm{~ms}$ and only after this time has elapsed is data analyzed. Figure $4.5 \mathrm{~b}$ and $\mathrm{c}$ show images of the bistable droplet states at equilibrium.

Depending on the amplitude of the pulse, four different dynamical behaviors are observed in experiment: i) small vibrations about a static shape; ii) bi-stable oscillations that toggle up, down and so forth n-times before coming to rest (up to 


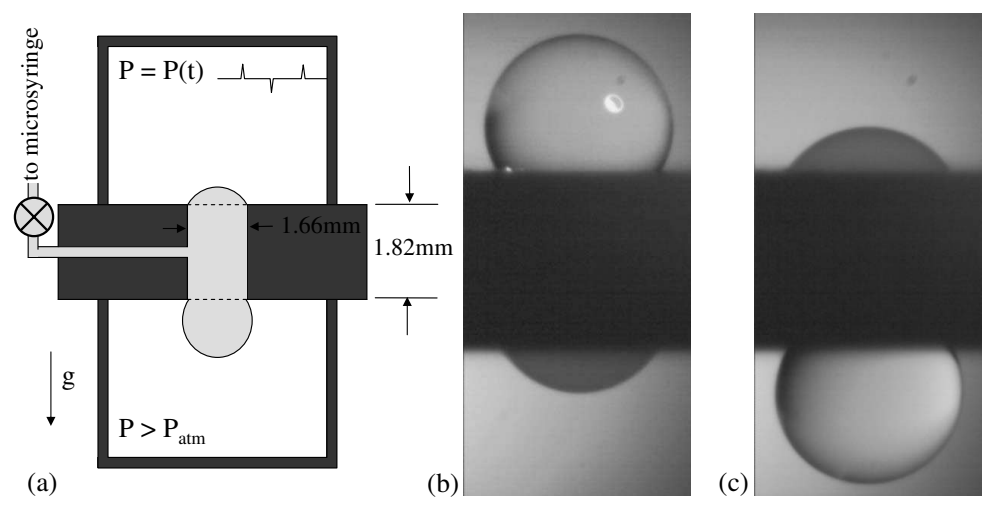

Figure 4.5: a) Experimental setup for droplet system with a steady pressure bias in the lower chamber and transient applied pressure pulse in the upper chamber. Images of the states when the system is bi-stable where b) is before and c) is after a positive pulse $P(t)$ is applied.

$n=5$ ); iii) deformations that blow the liquid completely out of the tube and iv) deformations where some liquid is left in the tube with the rest breaking off as a satellite droplet. A high speed video camera is used to capture the droplet motions (Redlake MotionPro HS2-C-4). For large pressure pulses the initial shape deviations of the droplets are not spherical caps (higher capillary modes are excited). However, later in the large-pulse decay and for the entire decay to equilibrium of small-pulses, the spherical-cap assumption holds reasonably. Image capture with edge detection gives the droplet volumes and thereby the reported $\Theta$ and $\lambda$ from experiment.

Time traces $\Theta(t)$ show damped oscillations. The role of dissipation is clearly seen in the experimental phase-plane plot corresponding to case ii), figure 4.6a. The under-damped nature of the oscillation is evident for the small disturbance case i), figure $4.6 \mathrm{~b}$, where a large number of vibrations occur before the droplet 
(a)

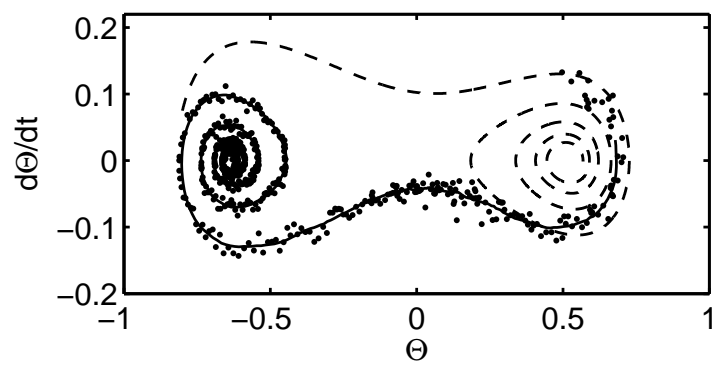

(b)

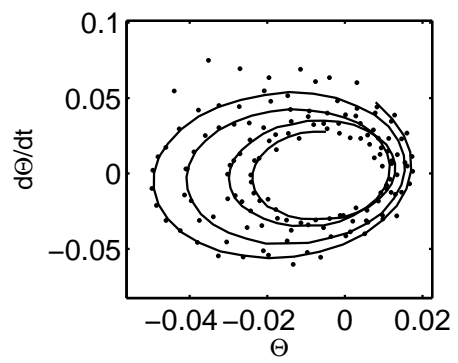

Figure 4.6: Phase-plane plots of experimental data as single frames (dots) and as timeaverages over many frames (solid curves): (a) for a large disturbance that causes a toggle from droplet-down to -up (volume $\lambda=1.2$ ) and a solution of the viscous model (dashed curve) starting from initial condition of comparable magnitude (displaced for decay to the opposite equilibrium, for clarity); (b) for a small disturbance that causes the droplets to vibrate about the steady state (volume $\lambda=0.4$ ) where the center is slightly off-set from $\Theta=0$ due to imperfect countering of gravity by the pressure bias.

settles to equilibrium. For these, by fitting to $\exp (i \omega t) \exp (-\delta t)$ (dimensional), it is straightforward to extract the frequency $\omega$ from the trace. Frequencies are plotted in figure 4.4a with an experimental uncertainty of a few per cent. Damping rate $\delta$ can also be extracted but has a greater relative error, as dissipation is a lower-order

effect. For completeness, we report that the damping $\delta \sim 20 s^{-1}$ is found to be constant to within $15 \%$ over the $\lambda$ range. Setting $\delta=(\mu / \rho) / \ell_{\nu}^{2}$ and solving for the viscous length-scale gives $\ell_{\nu} \sim 0.2 \mathrm{~mm}$.

\subsection{Discussion}

The dynamics of a capillarity-generated center-of-mass mode has been considered in a number of contexts, including the pendant free droplet (DePaoli et al. (1994)) 
and the sloshing of liquid mass in a cylindrical tube (Bian et al. (2003)). Strani \& Sabetta (1984) considered a droplet in contact with a solid spherical-cap support - not unlike the contact that a 'golf tee' makes with the ball - and made a detailed comparison with Rayleigh oscillations. The droplet was surrounded by a second liquid (immiscible). The same equations that Rayleigh solved were augmented with appropriate boundary conditions for the contact. They found that all the Rayleigh frequencies were modified. In addition, the constraint introduced a new low-frequency mode. This mode tends to a zero-frequency rigid displacement as the contact diminishes and tends to infinite frequency as the contact envelopes the entire surface. Hence, there is a cross-over for Strani's problem. Indeed, to the extent they can be compared, all our linear frequency results are qualitatively consistent with Strani. An important difference is that Strani's hemispherical meniscus has non-zero frequency. This is due to the asymmetry of the constraint and precludes a quantitative comparison.

The linear frequencies we report reflect a balance between inertia and the capillary restoring force, as modified by the constraint. Increasing $L$ generally increases the inertia of the system and decreases the oscillation frequency, as illustrated by the linear results (4.8) and (4.10). In particular, for lens-shaped interfaces on a tube (4.8), in the limit of planar shapes $\lambda \rightarrow 0$, the restoring force is constant and inertia controls the frequency, $\omega_{1}^{* 2} \rightarrow 8 / L$. The dependence on $\lambda$ is more subtle. As $\lambda$ increases, the frequency monotonically decreases to zero at the hemispherical state. In fact, all constrained hemispherical shapes $\lambda=1$ have zero linear frequency. The reason for this is that the potential-well is flat to second-order at this state - it transitions here from concave-up to concave-down along the family of symmetric states. Along the anti-symmetric family (4.10), the linear frequencies 
increase from zero, at $\lambda=1$, reach a maximum and then decrease back down to zero, as $\lambda \rightarrow \infty$. The maximum is an artifact of the scaling, however (relative to inertia, capillarity decreases due to increasing volume). Indeed, rescaling by $\omega_{2}$, cf. (4.1), shows a monotone increasing frequency from the hemispherical shape, according to,

$$
\left(\omega_{2}^{*} / \omega_{2}\right)^{2}=\frac{3}{16} \frac{(y+1 / y)(y-1 / y)^{2}}{(y+1 / y+3 L)} \text { for } y>1 .
$$

Hence, there is only one $\lambda_{c}$ below which the constrained sphere $(L=0)$ has lower frequency than the unconstrained one (same volume). The value $y_{c} \sim 2.68$, reported in Section 4.1, corresponds to $\lambda_{c} \sim 3.55$ and we see that all the measured frequencies are below the lowest Rayleigh frequency (figure 4.4a).

For completeness, $\omega_{1}^{*}$ can also be scaled with the Rayleigh frequency $\omega_{2}$ corresponding to the same volume,

$$
\left(\omega_{1}^{*} / \omega_{2}\right)^{2}=\frac{3}{4} \frac{y\left(y^{2}+3\right)\left(1-y^{2}\right)}{\left(y^{2}+1\right)^{3}(2 y+3 L)} \text { for } y<1
$$

Note that, in this scaling, the limit $\lambda=0$ is a singular limit due to the vanishing of the scaling volume. This form may nevertheless be useful to the practitioner.

Finite-amplitude oscillations can have frequencies that are higher or lower than their linear counterparts, as predicted in figure 4.4b. The softening/hardening can be understood in terms of the nonlinearity of $C(\Theta)(4.7)$ which shows softeningspring behavior for smaller volumes and hardening for volumes nearer hemispherical. Alternatively, the effect can be seen in the potential $U(\Theta)(4.12)$ and how its landscape changes with $\lambda$.

So far, relevance of the model is based on comparison to measured frequencies. Linear frequencies are in quantitative agreement over a decade of volumes (figure 4.4a). The range of volumes tested in experiment is limited by the contact 
pinning. As we shall now explain, it turns out that measured viscous dissipation is also consistent with (4.3) written in terms of $\Theta$; that is, with the viscous extension of $(4.5)$,

$$
\frac{d}{d t}\left(A(\Theta) \frac{d \Theta}{d t}\right)+C(\Theta)=18 R e^{-1} L f\left(\frac{d \Theta}{d t}, \Theta\right) .
$$

In contrast to $A$ and $C$, the dependence of $f$ on $\Theta$ and $d \Theta / d t$ is not known. Nevertheless, for the linearized version of (4.16), which governs the decay of smallamplitude disturbances, there is only a single unknown coefficient $f_{*}$,

$$
A_{*} \frac{d^{2} \Theta}{d t^{2}}+C_{*} \Theta-18 R e^{-1} L f_{*} \frac{d \Theta}{d t}=0 .
$$

The damping of (4.17) can be fit to the measured $\delta \sim 20 s^{-1}$ (Section 4.3) to find $f_{*} \sim 3.4$. A criteria for neglecting viscous effects emerges. That the coefficient of the damping term be small relative to that of the inertia term in (4.17) requires, Re $\gg 60 L / A_{*}$. For the range of $\lambda$ where there are measurements, $A_{*} \sim 5$, and the criteria becomes $R e \gg 13$. For water and our tube size, $R e \sim 240$, suggesting that inertia dominates but that viscous effects are nonetheless non-negligible - all consistent with observation.

Viscous influence has been measured in the context of small disturbances from equilibrium. In an ad hoc fashion, we now replace the nonlinear viscous force in (4.16) with the linear term $18 R e^{-1} L f_{*} d \Theta / d t$. That is, the nonlinear evolution equation (4.5) is generalized to include linear viscous effects. This damped nonlinear ODE is solved starting from an intial condition comparable in magnitude to that for the experiments to obtain the trajectory seen in figure 4.6a. To sum up, not only does the finite-amplitude behavior exhibit dynamics in qualitative agreement with the inviscid model (figure 4.6), the influence of dissipation is captured (with no free parameters) by a linear damping term with coefficient measured from small-amplitude data. 
In comparing experiment to model, the neglect in the latter i) of gravity, ii) of deformations that are not spherical-shaped, and iii) of viscosity are the three most significant idealizations. Viscous effects iii) have just been discussed. Regarding i), the pressure bias applied in the experiment serves to adjust the position of the zero-reference for the hydrostatic head but does not eliminate shape distortions due to gravity. Including gravity as a perturbation from zero Bond number would be a straightforward extension - one that might destroy much of the simplicity of the present model, however. Regarding ii), the restriction to spherical-cap deformations is reasonably valid for small disturbances. For large pressure pulses, the droplets clearly deform from spherical shapes and can take the form of pendent drop oscillations, similar to those reported in Basaran \& DePaoli (1994). These higher-frequency secondary vibrations may influence how fluid is exchanged between the droplets. However, these modes quickly dissipate (usually within the duration of the pressure pulse, $100 \mathrm{~ms}$ ) and the spherical-cap shapes recover.

In summary, the different dynamics predicted by a capillarity/inertia model with spherical cap deformations are faithful to observation. Viscous damping (included as a model extension) and non-spherical shapes are lower-order effects for the parameters considered.

\subsection{Acknowledgement}

Support by NASA NAG3-2713, -2714, NSF DMI-0500311, -0500408, ARO and DARPA is gratefully acknowledged, as are discussions of PHS with Liz Marcil regarding modeling. 


\subsection{Appendix*}

\subsubsection{Nonlinear effects of restoring capillary pressure}

Much of the bending in the frequency amplitude relationship can be explained by the nonlinear restoring pressure. Figure 4.7 plots $C(\Theta)$ vs $\Theta$ for different values of $\lambda$, where a) shows results for $\lambda<1$ and $b$ ) shows results for $\lambda>1$. Note that there is only one zero point in a) and three in b). For the same disturbance size in terms of volume difference, the restoring pressure is larger for small values of $\lambda$, agreeing with the predicted high vibration frequency. The nonlinearity of the curve agrees with the trend in Figure $4.4 \mathrm{~b}$ ). That is, for small $\lambda$ the the value of $C(\Theta)$ decreases from the linear behavior. For larger values of $\lambda$, the the value of $C(\Theta)$ increases from the linear behavior.

\subsubsection{Analytic frequency amplitude approach}

We can extend the analytical result to obtain the frequency / amplitude relationship for finite disturbances with $\lambda<1$. The approach that we use is to scale time by the natural frequency, $\omega_{0}$, given by Equation (3.24). Then expand time in the power series expansion using Lindstedt's method. The results gives

$$
\omega=\omega_{0}\left(1+k_{1} \Theta+k_{2} \Theta^{2}+\ldots\right)
$$

where $\Theta$ is the amplitude of the disturbance. It turns out that $k_{1}=0$ and $k_{2}$ is given as

$$
k_{2}=\frac{1}{8}\left(\frac{\left(10 H_{0}^{5}+9 L H_{0}^{4}-36 H_{0}^{3}-54 L H+H_{0}^{2}+2 H_{0}+9 L\right)}{\left(2 H_{0}+3 L\right)\left(H_{0}^{2}-1\right)\left(H_{0}^{8}+4 H_{0}^{6}+4 H_{0}^{2}+1\right)} .\right)
$$

\footnotetext{
${ }^{*}$ Section 4.6 has been added to the original manuscript.
} 

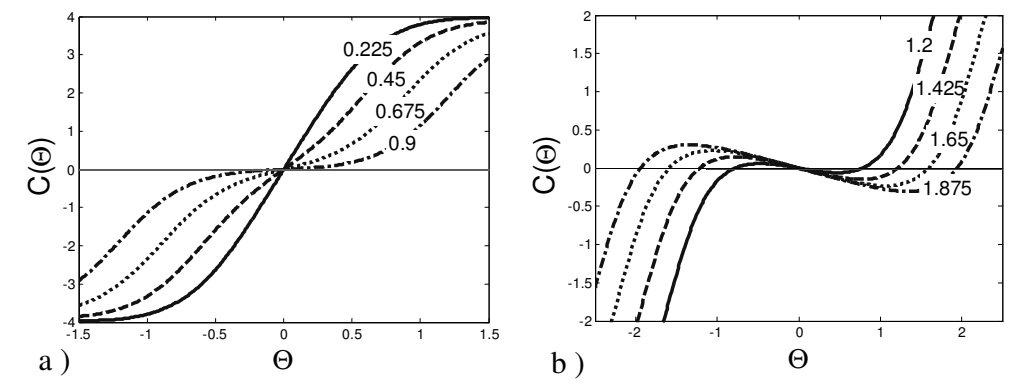

Figure 4.7: a) Plot of pressure difference, $C(\Theta)$, vs $\Theta$ for various values of $\lambda<1$. Note that there is only one zero and the nonlinearity in pressure b) For values of $\lambda>1$ there are three zeros in $C(\Theta)$.

A similar approach could be used to determine the frequency / amplitude relationship for finite disturbances with $\lambda>1$, although the results are not given here. 


\section{Chapter 5}

\section{Apparatus and Experimental Procedure}

\section{$5.1 \quad$ Overview}

This section addresses the changes to the casting process that have occurred in the Olin Hall 101 laboratory from 2001 to 2006. It also discusses some of the experimental observations that are still not fully understood. Section 5.2 discusses the modification to the superstructure which provide greater alignment ability. Section 5.3 describes the hill-and-valley profiles of the casting wheels. The data acquisition system was also upgraded as discussed in Section 5.4. A non-contact gap sensor was added in the summer of 2005. Section 5.6 addresses the use of a laser to modify the substrate through the cast. The laser is mounted on a 3 -axis translation table for alignment purposes. The operation of this table is also discribed. Finally, section 5.7 discusses some of the common experimental problems that lead to cast failures.

\subsection{Superstructure modification}

The superstructure was redesigned to allow for easier and safer casting. The redesign allows for finer control of the degrees-of-freedom for the alignment of the crucible by using micro-positioners to level the gap spacing on the left and right sides of the wheel and threaded bolt screws to level the gap spacing from the back and front sides of the wheel. Figure 5.1 a) shows the previous superstructure that was used until the summer of 2003. The current version is shown in Figure 5.1 b). Note the smaller height makes it easier to access the crucible area when the 

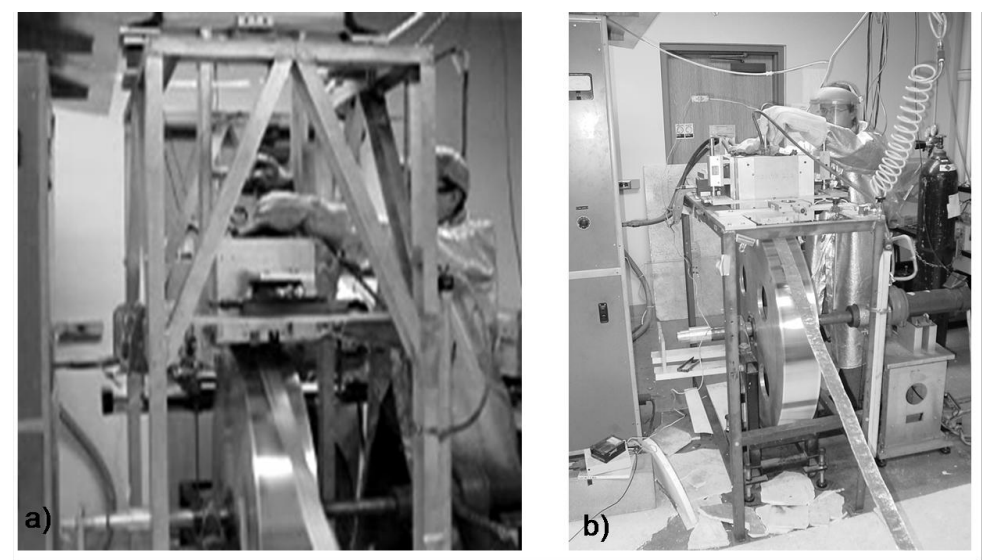

Figure 5.1: a) Experimental caster used until summer 2003. b) Modified casting superstructure machined to provide greater alignment capabilities.

operator pulls the stopper rod. Glenn Swan, of the Olin machine shop, made the modified structure.

The new design of the superstructure allows for very fine control during the alignment of the crucible. Due to the improved alignment, the time needed for setup has been decreased. The slim superstructure has also made the puddle more accessible, and hence the video capture of the puddle has fewer obstructions for lighting.

The box which houses the crucible has also been redesigned. It consists of a ceramic insulating material one inch thick enclosed by aluminum plates. The aluminum exterior makes future modifications to the box easier.

\subsection{Casting wheels}

There are three wheels that are available for casting: a $2.95 m$ circumference $\mathrm{Cu}-$ 0.4\%Be wheel, a $2.95 \mathrm{~m}$ circumference Steel wheel and a $1.91 \mathrm{~m}$ circumference 
$\mathrm{Cu}-0.4 \%$ Be wheel. They will be referred to as the large wheel, the Steel wheel and the small wheel, respectively. There are advantages and disadvantages to each. In Section 5.3.1 the topography of the wheel at room temperature is described. Then Section 5.3.2 shows how the topography of the wheel varies as it is thermally loaded during the cast. Table 5.1 lists the properties of each of the casting wheels.

Table 5.1: Summary of casting wheels and properties of each.

\begin{tabular}{cccc}
\hline $\begin{array}{c}\text { Material of } \\
\text { construction }\end{array}$ & $\begin{array}{c}\text { Circumference } \\
(\mathrm{m})\end{array}$ & Topography & $\begin{array}{c}\text { Amplitude of } \\
\text { eccentricity }(\mathrm{mm})\end{array}$ \\
\hline $\mathrm{Cu}-0.4 \% \mathrm{Be}$ & 2.95 & 3 Peaks and valleys & 0.25 \\
$\mathrm{Cu}-0.4 \% \mathrm{Be}$ & 1.91 & 1 Peak and valley & 0.05 \\
steel 4140 & 2.95 & 1 Peak and valley & 0.03 \\
\hline
\end{tabular}

\subsubsection{Wheel hill-and-valley profiles}

The large wheel has a smaller radius of curvature and hence is closer to a flat moving substrate. It also serves as a larger thermal sink due to the greater mass and therefore tends to heat-up at a slower rate than the small wheel. However, the out-of-roundness of the large wheel is the greatest. This may be due to a larger 'buckling' of the circumference of the wheel when thermally loaded, similar to the buckling of a loaded beam. The topography of the large wheel, measured using a mechanical run-out meter, is shown in Figure 5.2. A circumferential measurement is taken at 5 lateral positions along the surface of the wheel, spaced from the heater side of the wheel to the motor side. There is one large high spot and two smaller ones. The thickness variations of the ribbon per revolution are similarly greater than for the small wheel. Conversely, the small wheel heats-up more rapidly but has a smaller out-of-roundness. It also has only one high spot in the profile, as shown in Figure 5.3. Therefore the thickness variations per revolution are less, but 


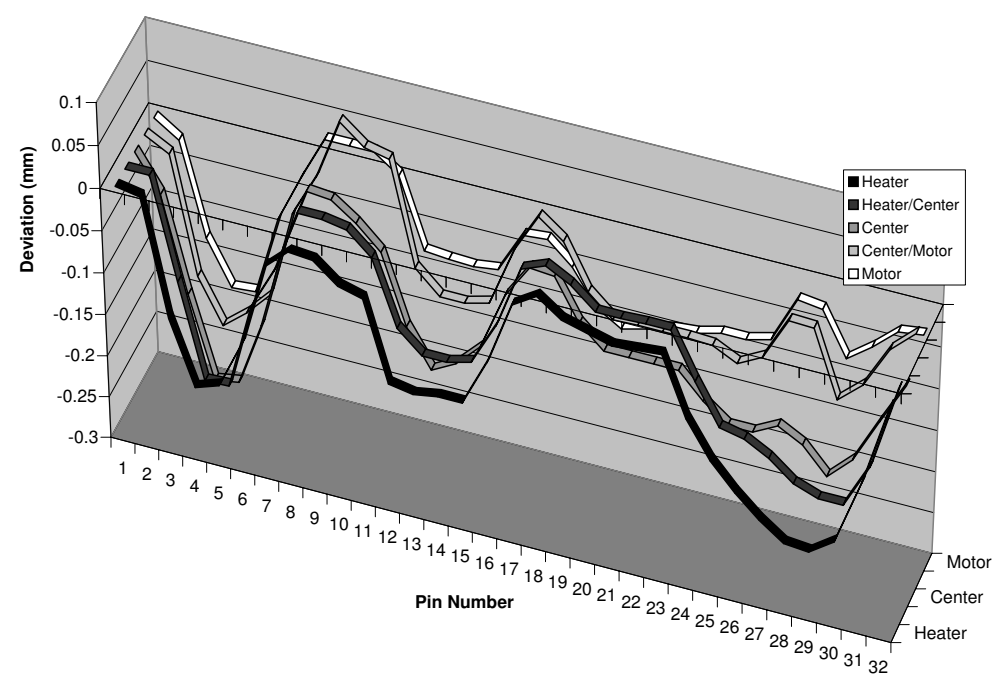

Figure 5.2: Profile of the large wheel showing the out-of-roundness with three peaks and valleys. This profile causes the gap height to vary periodically each wheel revolution.

the wheel heat-up is greater. This leads to a faster decrease in the gap height, due to the thermal expansion of the wheel. The overall decrease in ribbon thickness throughout the cast is greater on the small wheel.

The relative out-of-roundness of each wheel is shown in Figure 5.4. The measurement is taken along the center line of the wheel circumference. Note that a measurement is taken at each of the 32 pin locations which mount the surface of the wheel to the side plates. Each wheel has the same number of pins, even though the circumferences are not the same. The steel wheel has the least out-ofroundness of the three wheels. This is likely due to it being used to cast only twice. Each of the other two wheels initially had been machined to similar tolerances, but plastic deformation occurs after a number of casts. The profile of the large wheel, however, has not changed its over the years from 2001 to present (over 400 casts). 




Figure 5.3: Profile of the small wheel showing the out-of-roundness with one peak and valley. This profile causes the gap height to vary periodically each wheel revolution.

This may indicate that the deformation that occurs happens after the first few initial casts and thereafter the wheel does not further plastically deform.

Also, when casting large charge sizes, greater than $1.5 \mathrm{~kg}$, the ribbon can adhere to the wheel near the end of the cast. This has been purposely observed on a few casts where the intention was to study this phenomena. Scrapers were placed on the base of the wheel to prevent the ribbon from wrapping around the wheel and damaging the crucible. However, the reproducibility of the experimental conditions that induce sticking was poor. Sticking onset is believed to correlated with the wheel temperature however.

The ribbon tends to stick to the steel wheel after only a few revolutions. Only two casts have been done on this wheel because the bonding of the ribbon to the wheel is dangerous to the operators. The critical sticking temperature for a related system has been studied elsewhere (Maringer, 1988). The thermal diffusivity of 


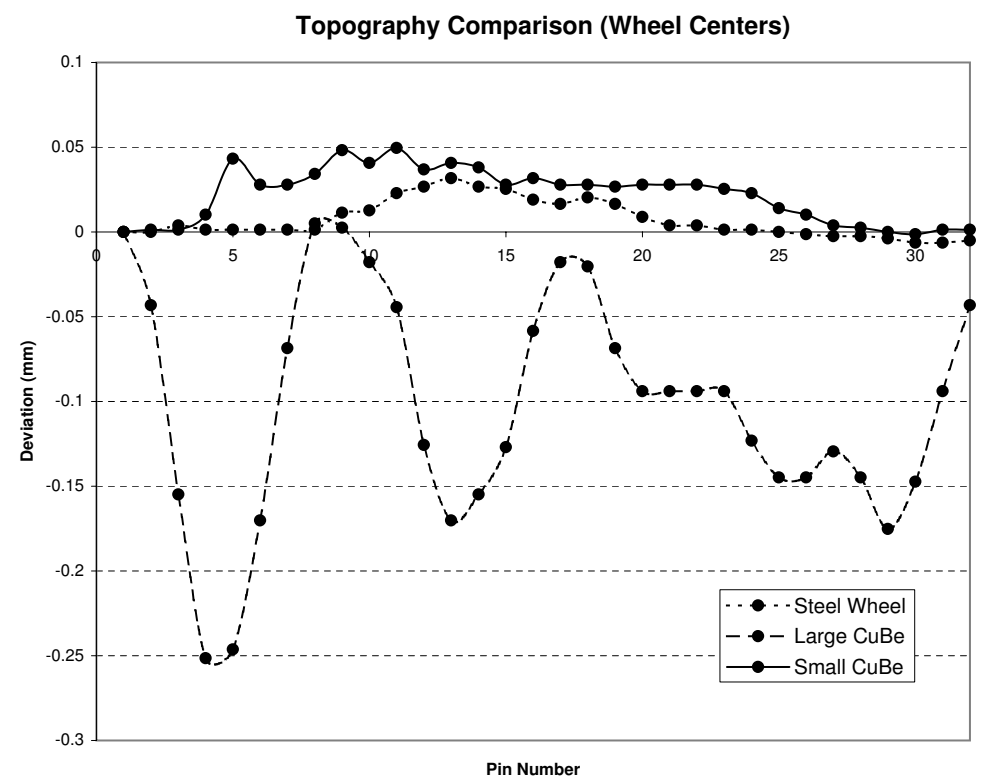

Figure 5.4: Profile comparison of the center of the three wheels showing the outof-roundness of each.

steel is about a tenth of that of copper. It is speculated that the heat transferred to the wheel therefore does not diffuse through the substrate to the same extent as for the copper wheel. The interface temperature is then hotter and sticking is more likely. Until a better control of this phenomena is achieved, it is best not to cast on this wheel.

\subsubsection{Wheel deformation}

The wheel profile changes as the cast progresses. Chapter 2 presented a simple model to account for the overall substrate expansion. However, the model does not account for how the out-of-roundness varies. Figure $5.5 \mathrm{~b}$ ) shows the gap run-out through the cast on the large wheel. This was measured with a mechanical run-out meter placed on the surface of the wheel. The periodic variation in the gap height is a template transfer of the cold profile from Figure 5.2 for the first few revolutions. 
Then the out-of-roundness appears to decrease and the run-out becomes more uniform. Near the end of the cast, the out-of-roundness increases again. Figure 5.5 a) shows similar trends in the ribbon thickness. It is difficult to determine whether the gap and thickness variation are in phase with each other from this data. The gap height was measured using a mechanical run-out meter. Again, one of the disadvantages of the large wheel is that the numerous gap variations (per circumference) lead to scatter in the thickness data. However, experiments on the small wheel indicate that they are indeed in phase, as discussed in Chapter 2. That is, when the gap is the lowest, the thickness is the greatest.

A simple mechanism for understanding the gap behavior is established by observing the thickness difference between the high and low gap cases. At a high gap, the ribbon is thicker, which means that wheel must absorb more heat compared to a lower gap. When more heat is absorbed, the wheel expands more. So the high gap narrows faster than the low gap leading to a more uniform gap and therefore more uniform ribbon thickness. It is not clear why the gap again appears to increase its out-of-roundness towards the end of the cast. A solid mechanics model of the deformation do to the nonuniform thermal loading might provide insight into the observed wheel expansion.

The small wheel also deforms as the wheel is thermally loaded. Figure 5.6 shows the gap run-out over time from cast ODSU06-22 and was measured with a non-contact capacitance gage. The thickness shows similar trends. It is interesting to notice how the wheel deforms. Initially the cold profile is observed with one high spot per revolution and two very small gap variations in between. The amplitude of the high spot decreases but the small gap variations begin to grow. Overall the 



Figure 5.5: a) Run-out measurement of the gap height through the cast showing that the substrate deforms as it thermally expands. b) The ribbon thickness showing the same trends as the gap. The data is from cast TBSU03-25. 


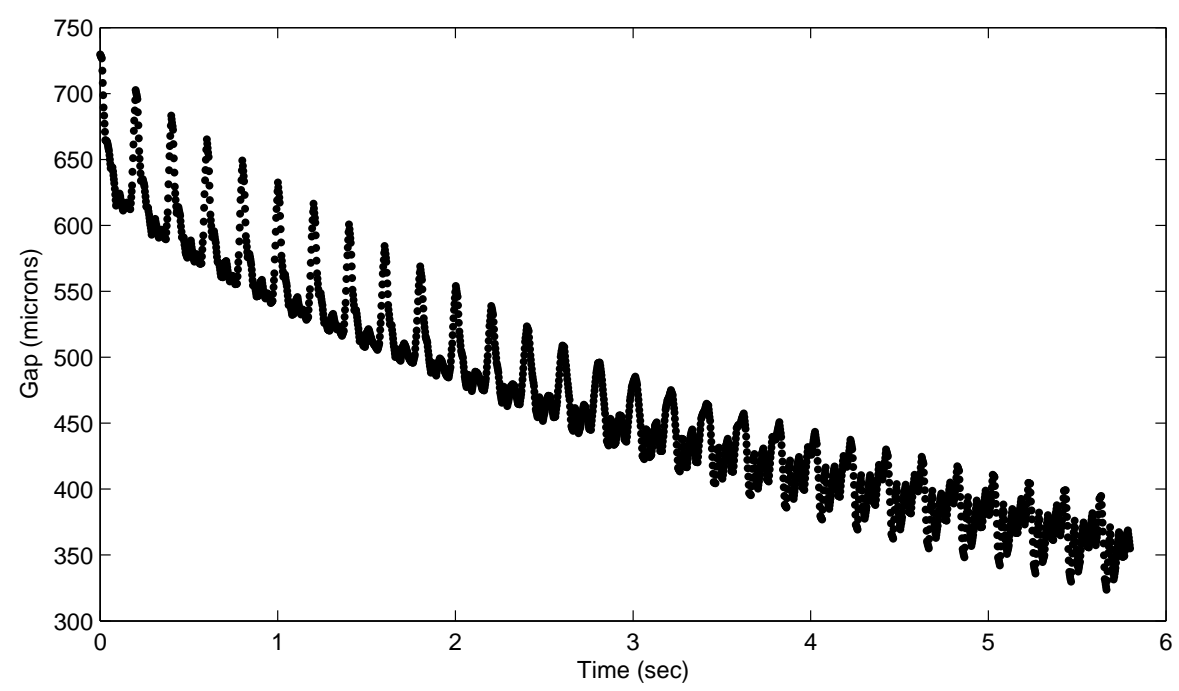

Figure 5.6: Run-out measurement of the gap height through the cast showing that the small wheel as it thermally expands and deforms. The data is from cast ODSU06-22.

gap becomes more uniform although more peaks and valleys form on the wheel. It is not clear why this occurs.

\subsection{Data acquisition system}

The data acquisition system measures the process parameters through the cast and controls a prescribed applied pressure ramp to the crucible. There is not a feedback control system in place during the cast. Rather, an applied pressure ramp is set beforehand by the operator to compensate for the decreasing metallostatic head of metal. The pressure is measured through the cast, but the pressure ramp does not compare the measured value to the desired value. A feedback control system is not practical due to the short casting times in our process (typically 5 seconds). 
The time dependent measurement of the processing variables are recorded with a data acquisition system (National Instruments card PCI-MIO-16E-4 and Labview software). Figure 5.7 shows the Labview program with the measurements after a completed cast. The data acquisition card is capable of 8 input channels and 4 output channels. Currently the input channels read (1) the crucible temperature, (2) the rpm sensor, (3) the applied crucible pressure, (4 and 5) the substrate temperature, (6 and 7) the gap sensor and (8) the trigger which begins the data acquisition. Only 1 output signal is sent - to control the applied pressure. All of the signals are read in as voltages and converted into their respective physical quantities.

The temperature of the substrate is measured using a fast-response K-type MedTherm thermocouple imbedded two $\mathrm{mm}$ below the substrate surface. An RPM sensor is placed on the axle of the wheel, which reads a voltage jump for each wheel revolution. A pressure sensor connected to the crucible measures the applied pressure and two non-contact runout sensors measure the gap height.

The MTI Instruments non-contact gap sensors are new additions to the data acquisition system. They act as capacitance gauges between a metal probe (model ASP-20-CTA Probe) and the wheel. An image is shown in Figure 5.8 (a). The signal is measured through an amplification box (AS-9000/2 Amplifier X2) shown in Figure 5.8 (b). It is possible to read two sensors simultaneously. The signal is then read into the Labview code.

\subsection{Data measurement}

The data recorded from each cast needs to be captured after each cast. This is a brief overview of how it is done. The data acquisition system records the 


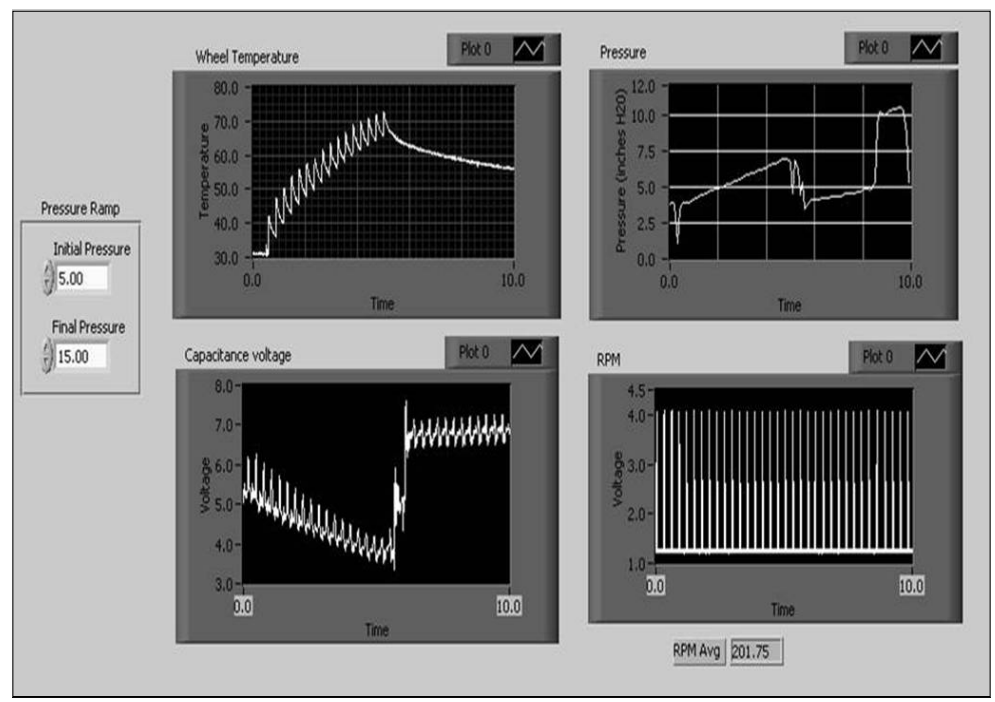

Figure 5.7: Data acquisition system implemented through Labview. The initial applied pressure and the final applied pressure are input parameters (far left window). The process variables, the wheel temperature(left top), the applied pressure (right top), the gap height (left bottom) and the rpm sensor (right bottom) are all monitored for ten seconds, although the cast ends after six seconds.

(a)

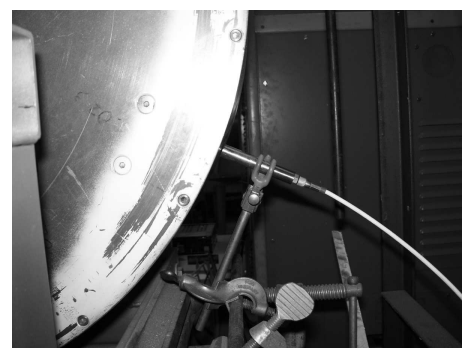

(b)



Figure 5.8: (a) The gap sensor is a small probe that is mounted approximately one $\mathrm{mm}$ from the wheel. (b) The measured voltage is read into an amplification box and the amplified voltage is read by the data acquisition card. 
wheel speed, the applied pressure, the gap run-out and the wheel temperature. The Labview program is set to take measurements at $100 \mathrm{~Hz}$. These values are transferred to an Excel spreadsheet, DataTemplate.xls. A best-fit for the pressure and wheel speed as a function of time are found. In the spreadsheet, the ribbon thickness needs to be entered in order to complete the data calculations. The ribbon is typically cut into $15 \mathrm{~cm}$ sections then measured for width and mass to give the thickness. The length of the piece sets the time-scale for the data. The wheel speed and the applied pressure are then evaluated at the times corresponding to the thickness measurement.

The overpressure is calculated as the sum of the applied pressure and the hydrostatic head of metal in the crucible. This is calculated through the cast based on the ribbon thickness. The mass of each piece is subtracted from the metallostatic head to adjust the overpressure calculation.

The gap height measurements need to be aligned with the thickness measurements. This is done by shifting the gap time-axis so that the maximum thickness is in phase with the maximum gap height. Experimentally it has been observed that the high spot on the wheel (lowest gap) always corresponds to the thinnest spot on the ribbon. Often the feeler gage, used to set the hot gap, leaves scratches on the wheel that get transferred to the ribbon. The gap data is then averaged using a window corresponding to the time scale of the ribbon thickness measurement.

The high speed video is transferred to the computer after each cast, typically as a video file (.avi). The video can be transferred to image files for further measurements. 




Figure 5.9: High frequency pulse laser used to template the substrate.

\subsection{Laser operation}

Attempts to thermally template the substrates during the cast have used a laser, model T40-Y70S-106Q (Spectra-Physics), to lay down 'hot-spots' in real time. The pulsed laser uses a Q-switched YAG rod for a high energy per pulse to provide a thermal template. The laser pulse frequency can be varied from 1000 to 10,000 Hz. At a casting speed of $10 \mathrm{~m} / \mathrm{s}$ this corresponds to a single pulse every $\mathrm{cm}$ to $m m$. The lower frequency laser pulses have higher energy per pulse than the high frequency setting. Figure 5.9 shows the caster with the laser positioned behind it.

An optical train with a focal lens is used to focus the laser spot to a point. The laser has been used in two ways to manipulate the ribbon. Figure 5.10 a) shows a schematic of how the laser is aligned with the focal lens to influence the puddle meniscus. The laser can be directed towards the middle of the meniscus (point A) or near the contact line (point B). Typically, the focal length is $5 \mathrm{~cm}$, but each lens is slightly different. Figure 5.10 b) shows a schematic of the laser being redirected 


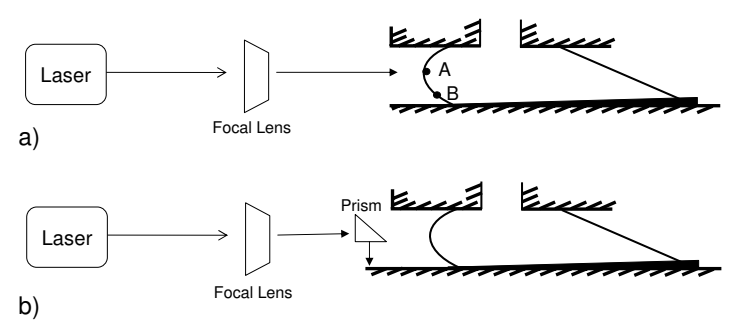

Figure 5.10: Optical train used to focus laser to a point. a) The laser can be directed at the puddle meniscus, either near the middle of the puddle (point A) or near the contact line (point B). b) The laser can be redirected through a prism to ablate the wheel.

with a prism to hit the wheel. The laser then ablates the wheel, leaving individual depressions on the wheel that gradually transition into a continuous trench as the substrate is exposed to discrete pulses over many rotations.

It is easier to ablate the wheel with the laser than to manipulate the meniscus. The most successful strategy is to lay a continuous line on the wheel by rotating the wheel and operating the laser until a visible line can be seen on the wheel. Directing the laser at the meniscus is difficult because the puddle moves throughout the cast, as discussed in Chapter 2. It is difficult to set the focal length of the lens to the right position since the puddle moves periodically and continually increases as the cast progresses. This leads to the laser only being in focus for a portion of the cast. An interesting phenomena has been observed when the laser is focused near the middle of the meniscus. Figure 5.12 shows a finger growing from the puddle towards the laser. It is believed that the laser leaves a hot spot on the meniscus which locally reduces the surface tension.

The laser is mounted onto a 3D translational table, as shown in Figure 5.13. Microstepping motors and controller software, provided by Applied Motions Prod- 


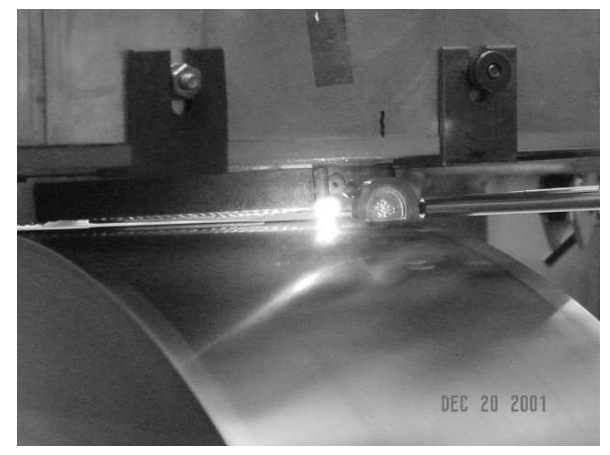

Figure 5.11: High frequency pulse laser focused with an optical lens and redirected to the substrate with a prism.

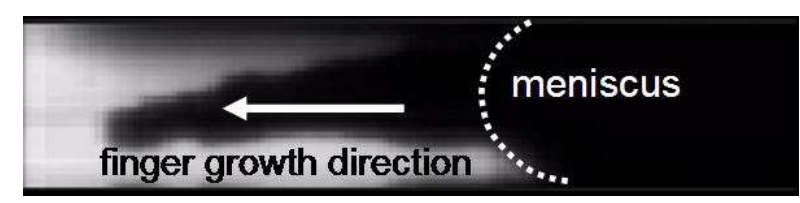

Figure 5.12: Focusing the laser on the meniscus directly leads to a finger growing from the puddle towards the laser. Image from Cast ID TBSU03-18. 


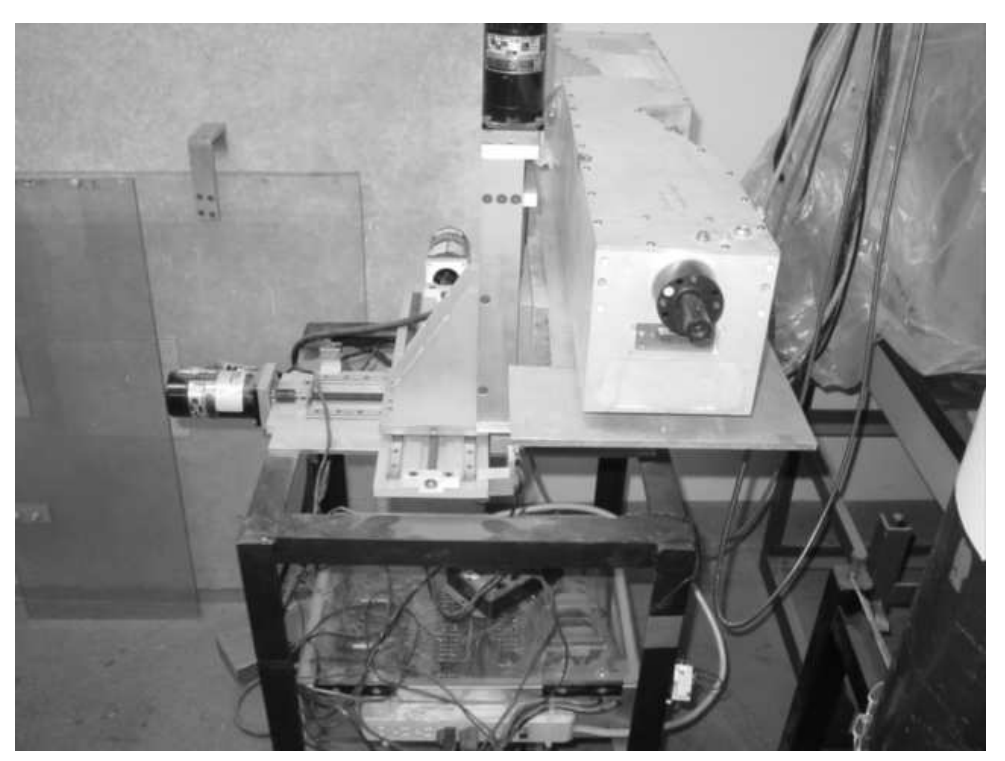

Figure 5.13: The pulse laser is mounted on the 3 stage translational table.

ucts, are used to position the laser. The $\mathrm{x}$ and $\mathrm{y}$-axis stages have a pitch distance of $0.5 \mathrm{~cm}$. These stepper motors are controlled through an electronic driver, model 1240i. The $\mathrm{z}$-axis stage has a shorter pitch distance of $0.2 \mathrm{~cm}$ and has a driver model 3540i. The motors are capable of microstepping at 50,800 steps per revolution. The shorter pitch in the z-axis allows for very fine control in that direction, which is the most important for alignment of the laser.

The SiNet Hub programable software is used to position the laser. Figure 5.14 shows an image of the software interface. The user inputs which axis to move, whether the motion will be clock-wise or counter-clock-wise and how many revolutions to take. After the laser alignment is completed, the translation table is programmed to move out a specified distance during the heating of the crucible. 




Figure 5.14: SiHub software interface used to position the laser.

The laser is then returned to the original aligned position when the casting is ready to proceed.

\subsection{Common experimental problems}

Some typical problems arise in the laboratory, and they will be discussed here so that other researchers may benefit. Most importantly, the casting should be done on a regular basis. When the equipment is not used for long periods of time, there tends to be problems. For example, the induction heater may function intermittently when not used for an extended time. Regular casting also helps to keep the necessary casting supplies in stock.

An inspection of the induction coils should be done before each cast to make sure the insulation around the coils has not worn. The insulation prevents the coil 
from touching itself and prevents any contact between the coils and the crucible. The paint on the crucible should also be inspected before casting. The paint prevents the graphite crucible from oxidizing at high temperatures and greatly extends the number of casts that the crucible can withstand.

The stopper rod used to prevent the molten metal from flowing out of the crucible typically lasts for $\approx 30$ casts. The stopper consists of a ceramic tube (for inserting a thermocouple to measure the melt temperature) glued to a boron nitride $(\mathrm{BN})$ stopper. The stopper is machined from a rod of pressed BN, with a hole drilled into the top for inserting the ceramic tube. A ceramic glue is used for connecting the tube and the stopper and must be cured in stages. A droplet of glue is placed into the stopper and then the stopper is placed in the collar of the crucible. The tube is then inserted into the stopper and the top cap of the crucible is screwed in place. The glue needs to dry for one hour at room temperature, then is step cured for an hour at $100{ }^{\circ} \mathrm{C}, 200{ }^{\circ} \mathrm{C}$ and $400{ }^{\circ} \mathrm{C}$. The stopper rod is too long to fit in the melting oven. A 500 Watt flood light is placed in close proximity to the crucible for the initial heating to $100{ }^{\circ} \mathrm{C}$. The crucible is then moved into the induction heater for the next curing stages. If too much glue is used or the temperature is raised to quickly, the stopper has a tendency of cracking. Curing the stopper rod in the crucible helps align the assembly.

The applied pressure in the crucible can often fail. A metal plate with two tubes for connecting the argon gas is mounted to the top cap of the crucible. The seal between the cap and the plate breaks down over time and cannot hold a pressure in the crucible. A new seal can be put in place, but a new top cap may be needed after $\approx 100$ casts.

The data acquisition system may be accidently triggered early, which causes the 
pressure to ramp unexpectedly. For this reason, the Labview program is typically not set until a few seconds before the cast begins. The operator that is pulling the stopper rod may hear the pressure transducer ramping if the cast is triggered early. Removing the pressure tubes to the crucible will prevent the molten metal from pouring out prematurely and the data acquisition system can be reset. 


\section{Chapter 6}

\section{Future Work}

There are many aspects of the casting which have opened further questions that should be pursued in future studies. The following sections address these issues.

\subsection{Onset of cross-wave defect}

In Chapter 3 the cross-wave defect was studied experimentally and the physics of the puddle vibration was identified. An ongoing collaboration with Prof. Brian Thomas at the University of Illinois has identified that air captured between the puddle and the wheel leads to the crater-trough profile seen in the ribbon. However, the connection between the meniscus motion and the air capture event has yet to be made, as shown in Figure 6.1. Also, the onset of the capillary vibration is not understood.

It is not clear whether an instability at the contact line causes the entire meniscus to vibrate or whether the meniscus vibration leads to the contact line becoming unstable and capturing air. An argument for either could be made. Understanding the onset mechanism would certainly help clarify the issue. It does seem that the puddle has a natural frequency, set by the capillary time scale, at which it tends to vibrate.

Experimentally, the defect can be avoided by either lowering the gap height or slowing the wheel speed. This seems to be consistent with a contact line instability. The contact angle between a fluid advancing over a solid is known to be a function of the contact line speed. When the contact angle nears 180 degrees, air entrainment can occur. It is possible that this is the onset mechanism for the 


\begin{tabular}{|c|ccc|}
$\begin{array}{c}\text { Length } \\
\text { Scale }\end{array}$ & Phenomena & Result \\
$\mathbf{m m}$ & $\begin{array}{c}\text { Molten meniscus } \\
\text { motion }\end{array}$ & $\begin{array}{c}\text { Cross-wave } \\
\text { defect in foil }\end{array}$ \\
$\mu \mathbf{m}$ & $\begin{array}{c}\text { Heat-transfer } \\
\text { interuption } \\
\text { (air pocket) }\end{array}$ & $\begin{array}{c}\text { Crater / trough } \\
\text { foil profile }\end{array}$ \\
\hline
\end{tabular}

Figure 6.1: The connection between the meniscus motion and the cross-wave defect has been established (open arrow). The connection between air capture and the crater trough profile has also been established (open arrow). However, the connection between the meniscus motions and air capture is still not understood (shaded arrow).

cross-wave defect. That is, as the contact angle reaches a critical value, the contact line detaches from the wheel and air is captured. This serves as a disturbance to the meniscus which vibrates in response. The vibration then forces the contact line to capture air in a regular manner.

A useful set of experiments would be to measure the contact angle, using the high speed video, for casts where the wheel speed is progressively increased. Determining the contact angle vs contact line speed would be very helpful. It could then be determined whether the onset of the cross-wave defect corresponds to the contact angle nearing 180 degrees. There would be some issues setting up these experiments, as measuring contact angles can be difficult.

One could make a case that something internal to the puddle, such as a flow instability, causes the meniscus to vibrate. This is a possibility, but there are some experimental observations that confound the issue. The defect frequency is not a 
function of the overpressure, provided the overpressure is large enough to cause the puddle not to be pinned at the nozzle slot. So, it seems that the defect frequency is independent of flow rate. Casts have also been done where the overpressure is increased through the cast, rather than being held constant. No influence on the defect wavelength was observed.

Before the capillary time scale was identified, a favored vibration mechanism was vortex shedding in the turning region under the nozzle slot. The vortex shedding frequency is typically a function of the Reynolds number and is therefore inconsistent with the observation the the defect wavelength is independent of the flow rate. However, a series of experiments were conducted where the nozzle geometry was varied in an attempt to modify the flow under the nozzle slot, as shown in Figure 6.2. The results were inconclusive, however. Nozzle b) and c) showed a few casts with no defect present but this happened intermittently. It is also not clear how the flow profile in the puddle can influence the contact angle.

Understanding the air capture event would be helpful to learning the onset mechanism. It still may not fully explain the crater formation process. Presumably, a layer of air is pulled under the meniscus. This air bearing then becomes unstable and collapses. As it collapses the air layer forms a number of craters between the molten metal and the wheel. The craters are irregularly spaced in a line along the wheel. Modelling the air bearing collapse could also be helpful is understanding the defect formation mechanism.

\subsection{Herringbone defect}

The herringbone defect, as shown in Figure 6.3, is another common casting defect. It is similar to the cross-wave defect, but occurs at a smaller wavelength. The 

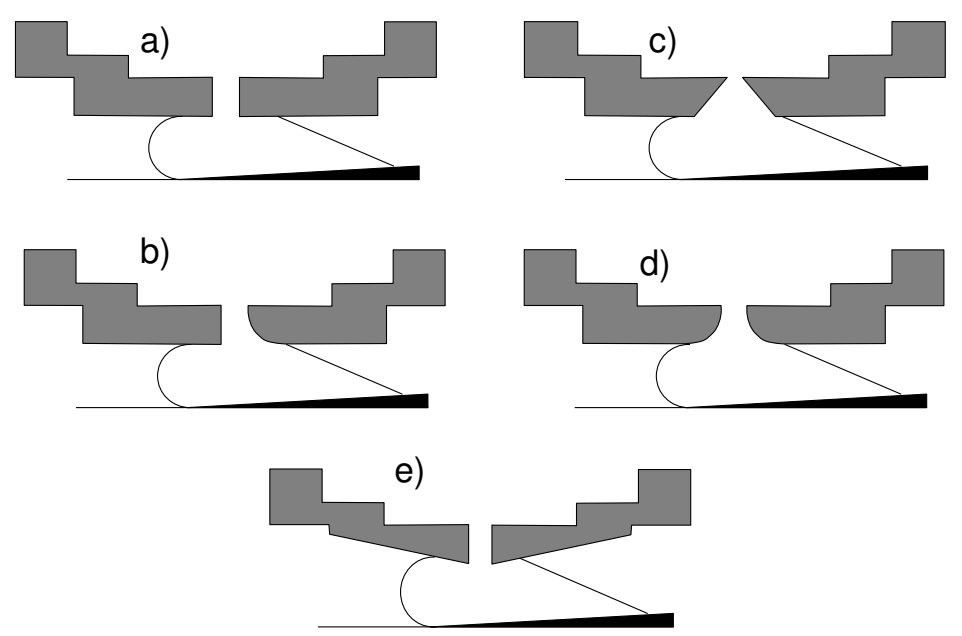

Figure 6.2: Nozzles with varied geometries were used in an attempt to alter the flow in the puddle. There were no conclusive results to indicate any influence on the cross-wave defect.

defect is registered on both sides of the ribbon. The frequency of the defect is on the order of $10000 \mathrm{~Hz}$, while the cross-wave defect is near $1000 \mathrm{~Hz}$. The herringbone defect is also common for pure Al casts, but the cross-wave defect does not occur for pure Al casts.

The herringbone defect occurs when the overpressure is low, so that the puddle is pinned at the nozzle slot, as shown in Figure 6.4 a). Again, this is contrasted by the cross-wave defect where the puddle is free to move, as shown in Figure $6.4 \mathrm{~b}$ ). It is not clear why pinning the puddle has this effect. The additional constraint placed on the puddle causes a defect to occur at a higher frequency.

There are two obvious things that one might ask about the herringbone defect. The first is whether there are any puddle motions that correlate to the defect. The second is whether the frequency follows the same scaling as the cross-wave defect. The EktaPro camera can only capture frames at $6000 \mathrm{fps}$, therefore it 


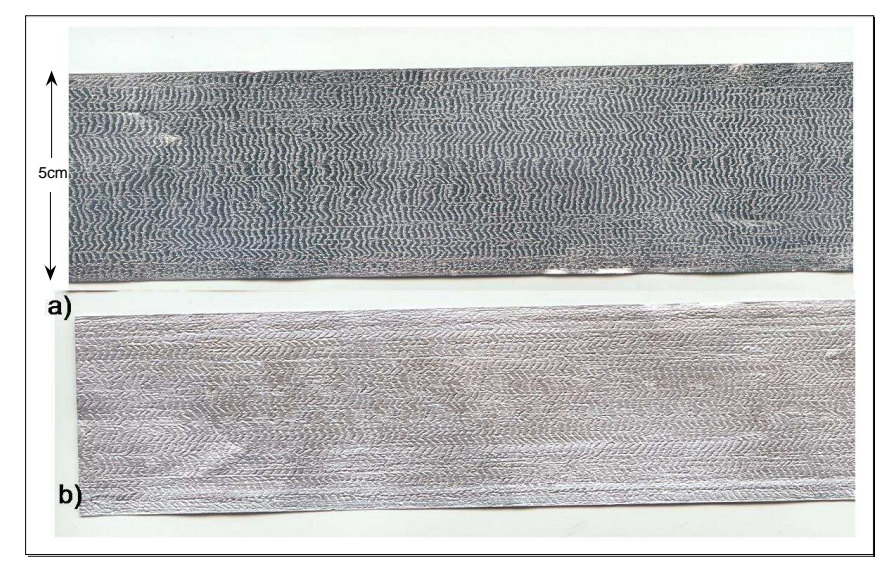

Figure 6.3: The herringbone defect where a) shows the wheel side and b) shows the air side. The defect is similar to the cross-wave defect but occurs at a wavelength near one $m m$. (Image from Cast ID TBSU05-16)
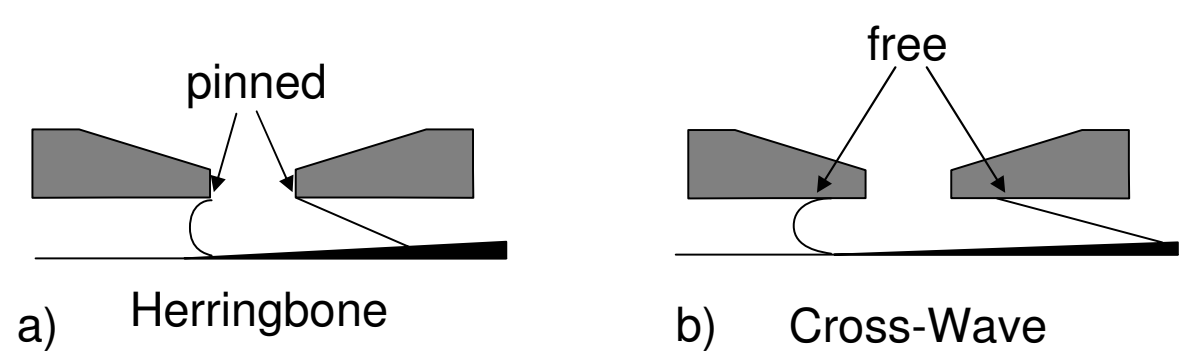

Figure 6.4: a) The herringbone defect occurs when the puddle is pinned at the nozzle slot. b) The puddle is free to move when the cross-wave defect occurs. 
could not capture any puddle motions at the herringbone frequency. However, a few experiments with a Phantom camera were done. The Phantom was used to get puddle data at 60,000 fps. No motions were observed at the herringbone frequency. However, the amplitude of the vibration may be too small to observe experimentally. The scaling of the defect has not been fully explored, in part because of the amount of time required to measure the frequency. The scaling for the few casts that have been measured is shown in Figure 6.5. The measurements are taken from only a single piece of each cast rather than a cast average. The linear fit to the data gives a slope of 6.9. This value should be taken lightly considering the small data set which it was taken from. Recall from Chapter 3 that the cross-wave defect had a slope of 1.94 .

Another interesting experimental observation is that when the overpressure is low and the puddle is pinned at the slot, the periodic variation in thickness is not observed. Presumably, since the puddle is pinned, the gap forcing does not change puddle length. That is, since the puddle has not completely filled the gap region, the periodic variation of the gap does not lead to the puddle motions described in Chapter 2. However, this has not been verified experimentally. Also, the low overpressure casts are of poor quality. They are very thin and often have pin hole defect as well as the herringbone defect.

\subsection{Streak formation}

The quality of the ribbon decreases as the cast progresses. Longitudinal streaks begin to appear on the ribbons, as depicted in the schematic in Figure 6.7. There is a large trough over the streak on the air side of the ribbon with a much smaller depression on the wheel side. The streaks do not have a characteristic wavelength 


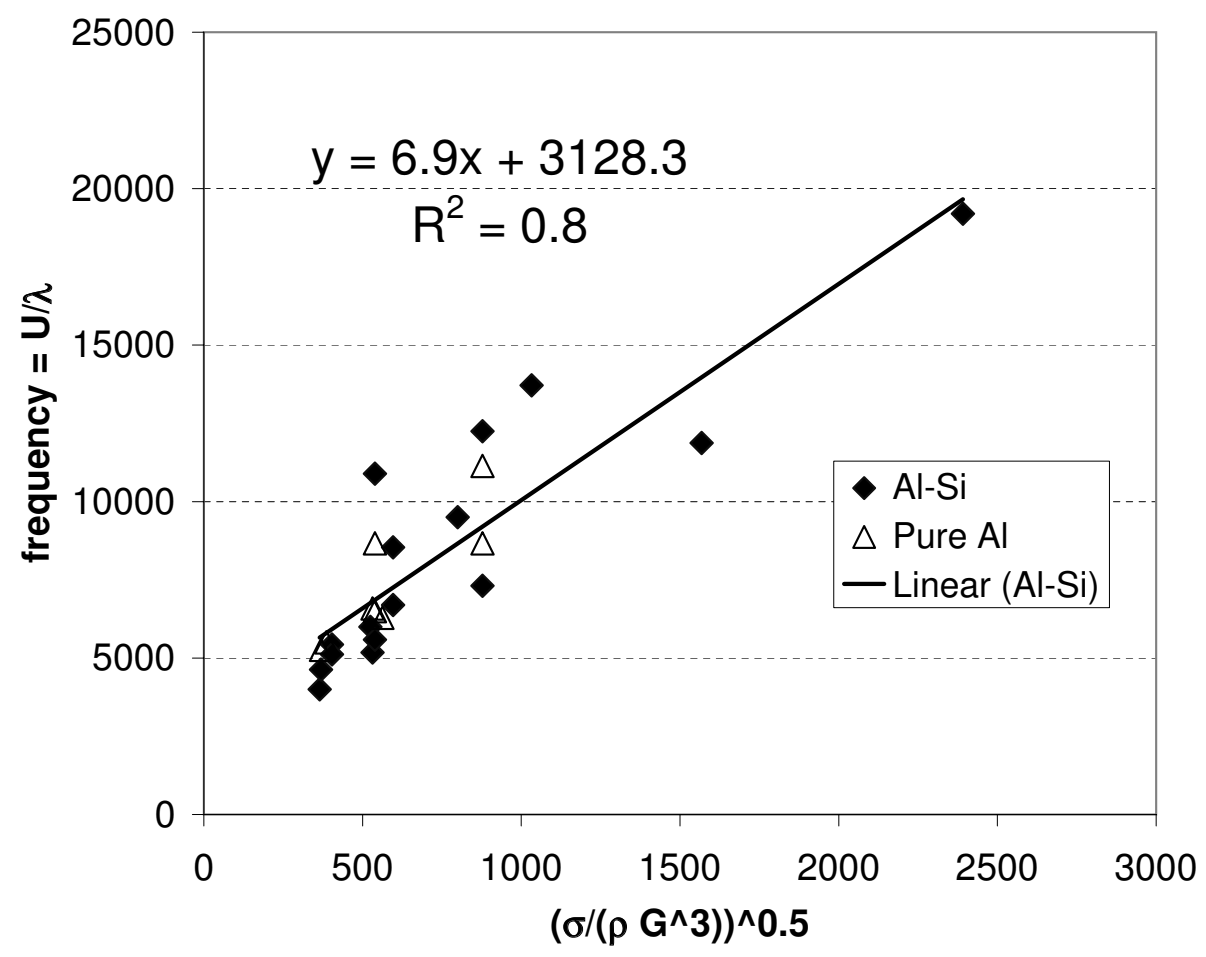

Figure 6.5: The herringbone defect frequency plotted against the capillary time scale. The linear fit is based on only the Al-Si casts and gives a slope of 6.9. (From casts TBSU04-21,28,TBSU05-23,26,31,33,39, and TBSP05-12) 


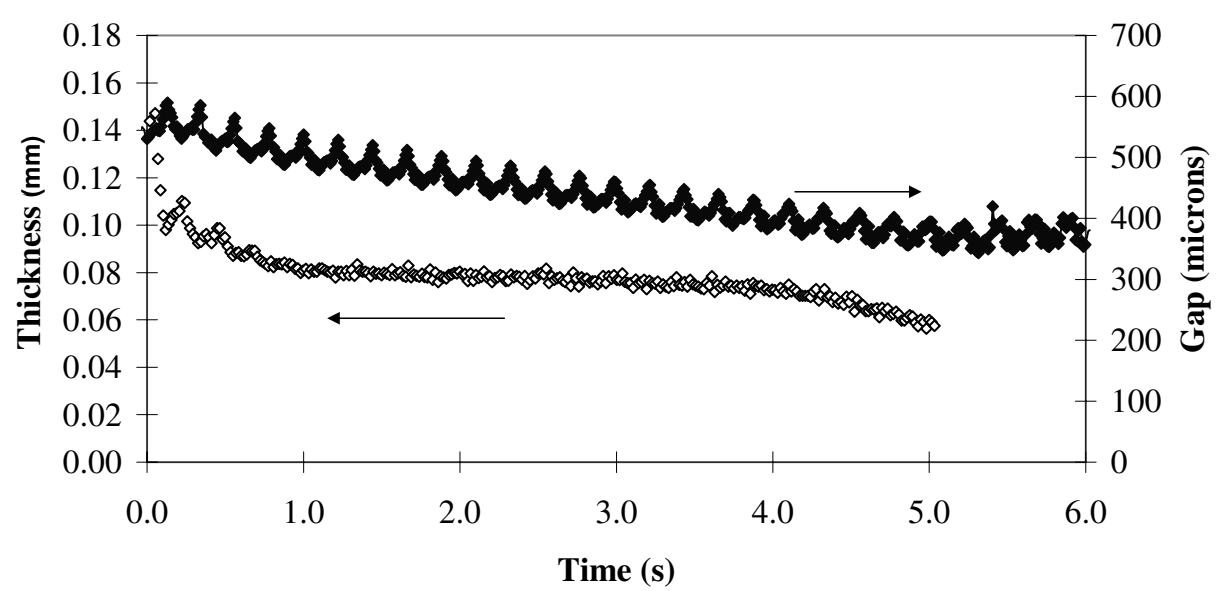

Figure 6.6: Under very low overpressure conditions, the puddle is pinned at the slot and does not respond to the gap forcing. The periodic variation in thickness is not observed. Data from cast ID TBSU05-63.

and the spacing appears random. Images from the wheel side and the air side of the cast are shown Figures 6.8 and 6.9, respectively. The transition is gradual with a few random streaks appearing then slowly increasing in number. The wheel side of the ribbon shows the streaks and is often discolored, showing a purple color. The air side has longitudinal thickness variations corresponding to the streaks below. The ribbon is brittle along the streaks and easily cracks if bent there.

To successfully cast the operating parameter must lie within an operating window for the process. A schematic of the operating window is shown in Figure 6.10. The axes are the Weber number $\left(W e=\rho U^{2} /(2 \sigma / G)\right)$ and the pressure index $(P I=\Delta P /(2 \sigma / G))$, as shown in Figure 6.10. Experimental and theoretical studies have mapped the operating window (Byrne, Weinstein \& Steen, 2006; Ibaraki, 1996). For both of these studies, the average values from the cast data were used; that is, there is one data point per cast.

The gap sensor allows us to get 'through-cast' data rather than using average 


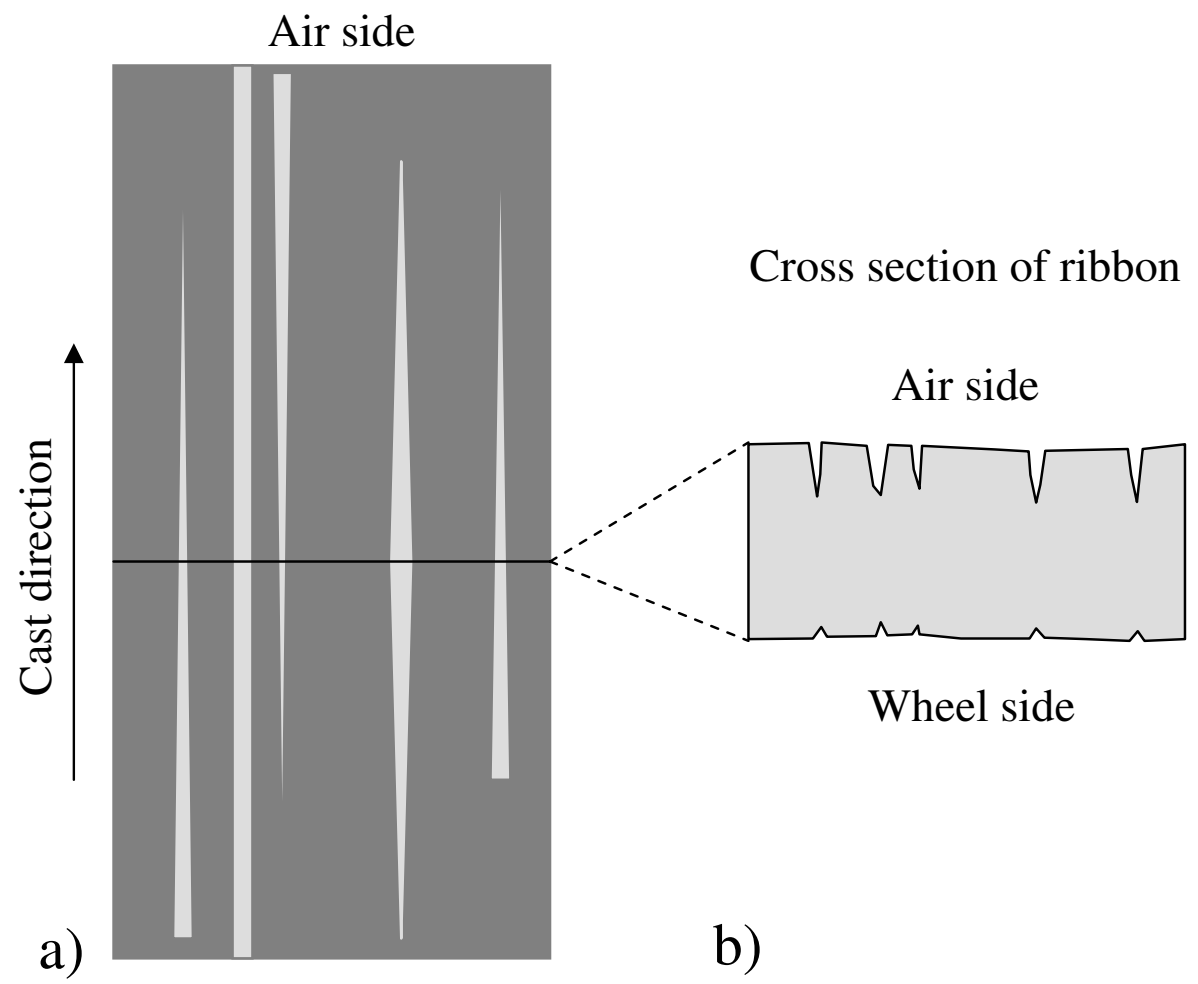

Figure 6.7: a) Schematic of the longitudinal streaks that begin to appear in the ribbon as the cast progresses. b) The streaks appear as a large trough on the air side of the ribbon with a much smaller depression on the wheel side. (Note that the horizontal length scale has been compressed for clarity. 


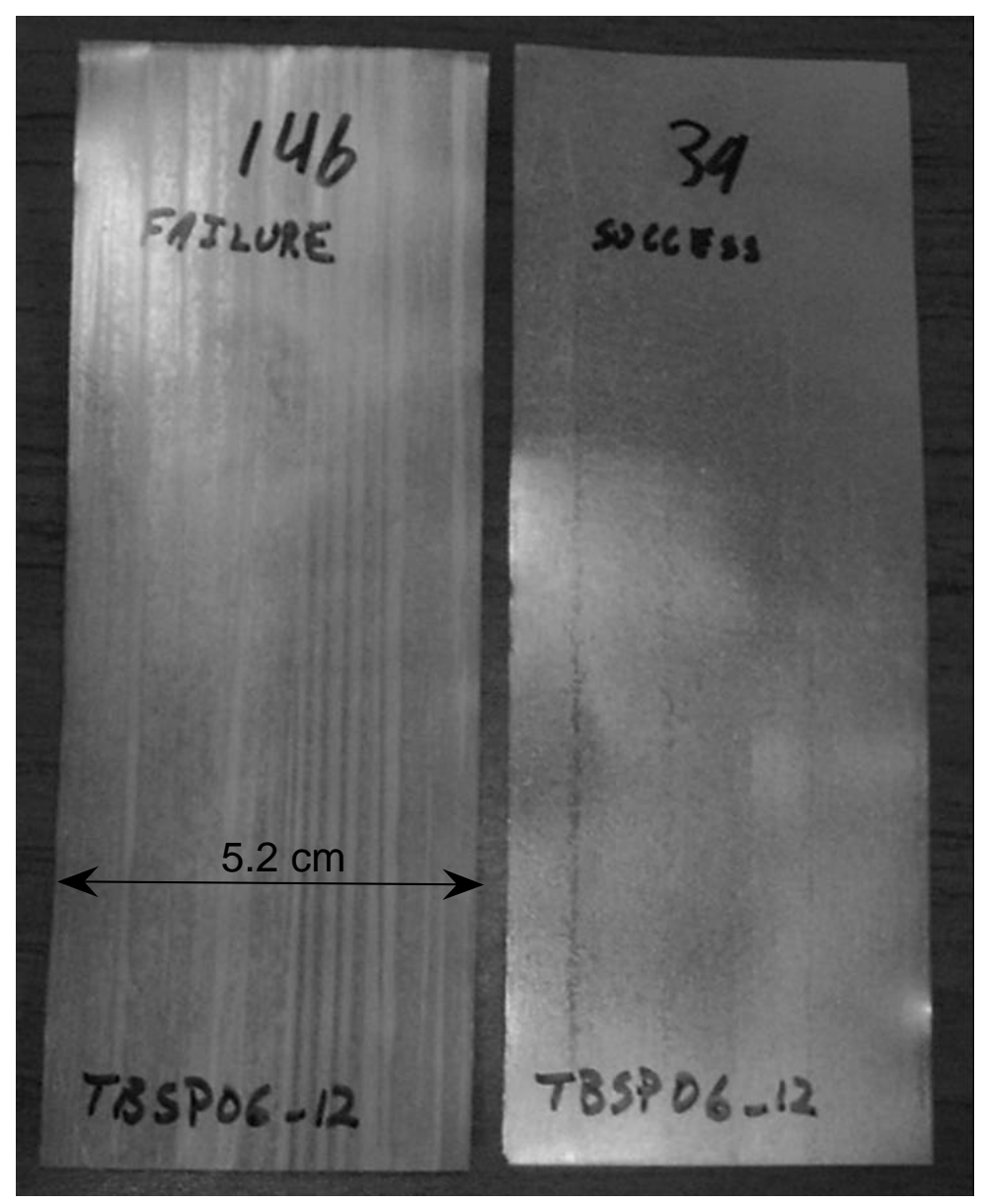

Figure 6.8: Streaks on the wheel side of the ribbon begin to appear in the ribbon as the cast progresses. The left image shows the wheel side of the ribbon with streaks and the right image shows a ribbon near the beginning of the cast. The images are taken from cast TBSP06-12. 


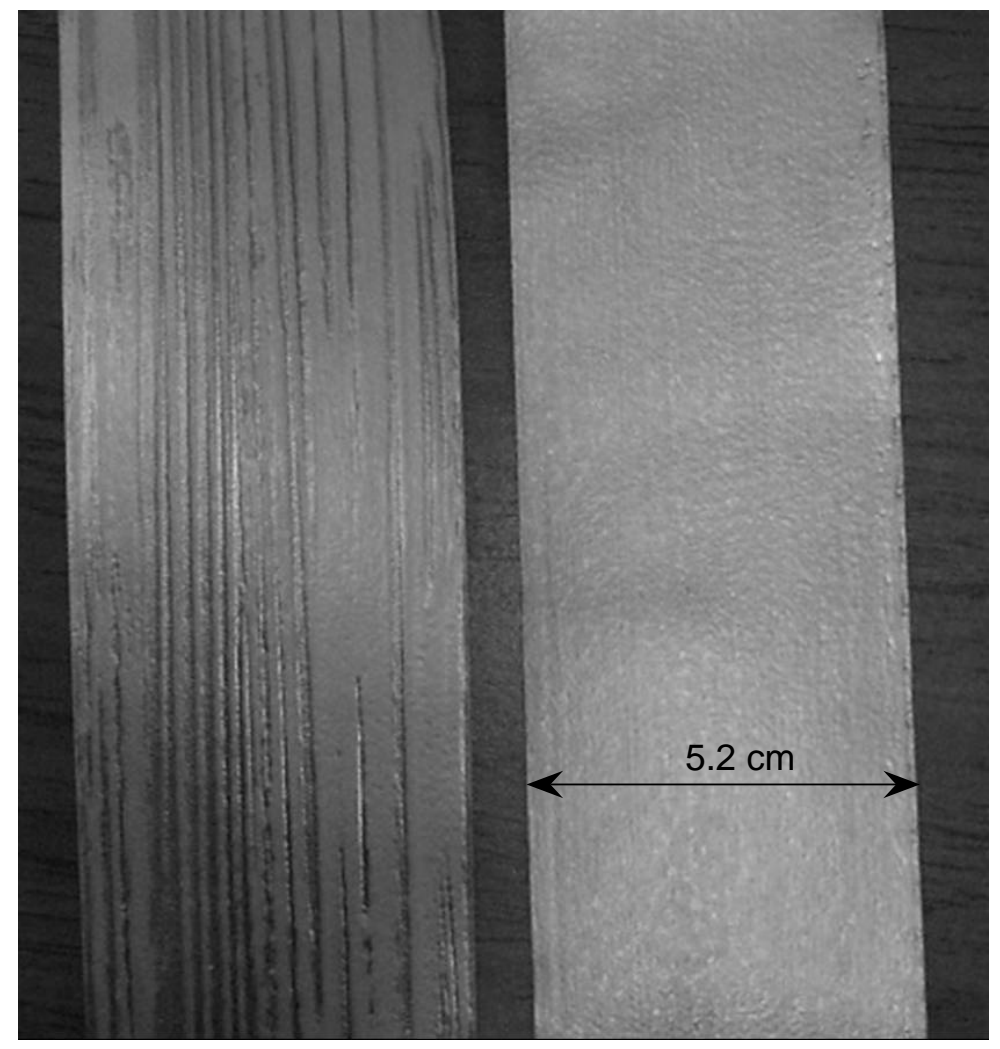

Figure 6.9: Streaks also appear on the air side of the ribbon as the cast progresses. The left image shows the air side of the ribbon with streaks and the right image shows a ribbon near the beginning of the cast. The images are taken from cast TBSP06-12. 


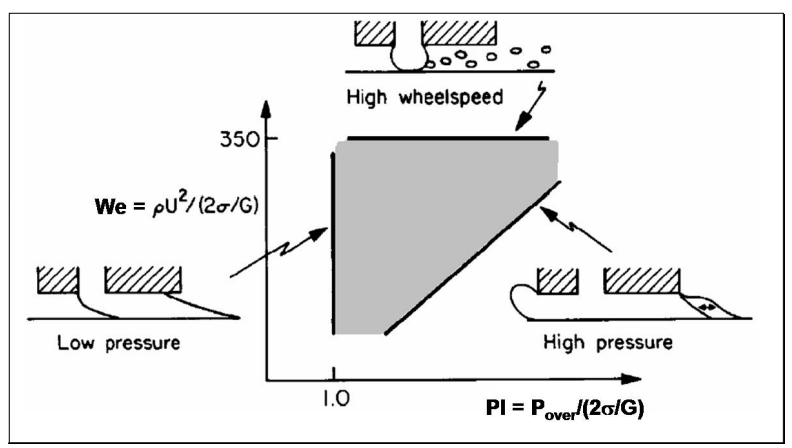

Figure 6.10: Operating window for the PFMS process indicating the limits for successful casting. [From (Steen \& Karcher, 1997)]

values from the cast data. The through-cast data gives many more data point per cast. Initially, it was thought that we could map the boundaries of the operating window by starting in a successful region and letting the variation in the gap height move into a failure region. Since both $W e$ and $P I$ are scaled by the gap height, the through cast data moves through the operating window with time. The streaking in the ribbon may be considered a cast failure. If this is the case, then the transition to the failure can often occur within the bounds of the operating window. Figure 6.11 shows the operating window with the through-cast data, indicating where the streaks start for each cast. The data is rather puzzling at first since there is no clear boundary for the onset of the streaking. Beginning a cast at conditions where streaks came in at the end of a different cast does not show streaks. The onset of these streaks are not captured by the $W e$ or $P I$.

\section{It is suspected that the streaks are related to the wheel temperature.} The streaks occur sooner in the cast on the small wheel. This may be due to the small wheel heating up more rapidly than the large wheel. To account for the wheel heat-up, an additional parameter is added to the operating window that 


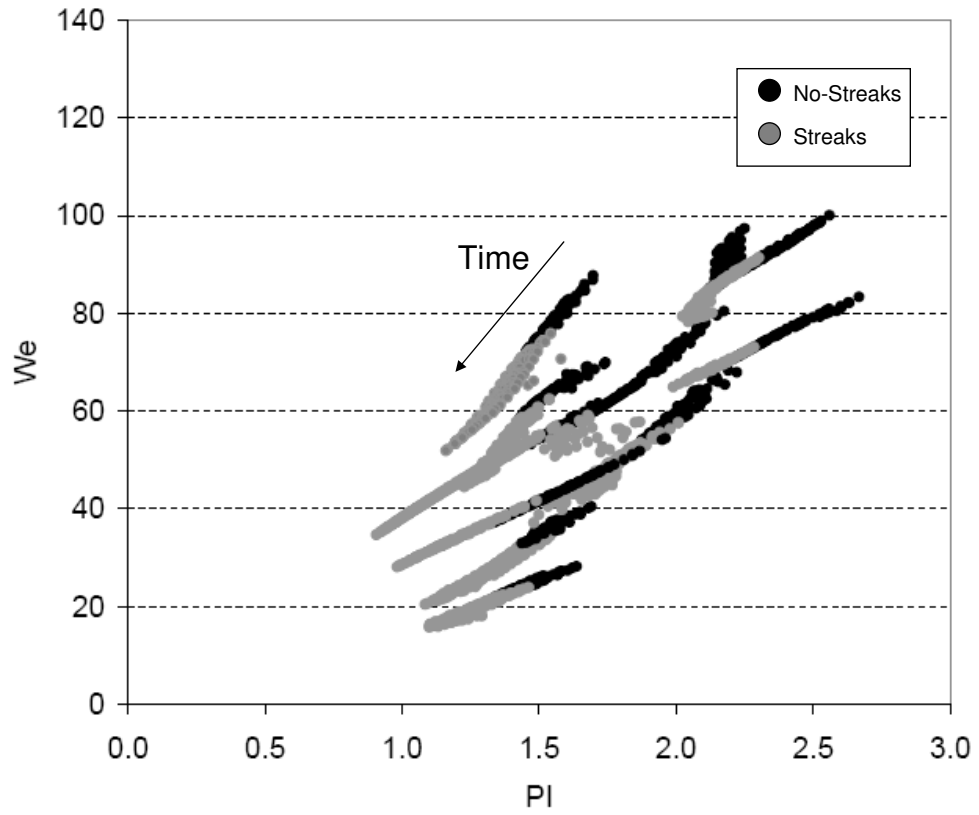

Figure 6.11: Operating window using through cast data indicating where each cast transitions to the streak defect. Data from casts TBFA05-11,12,14, TBSU0560,61,62,63,64,65, and TBSP06-12,13. 


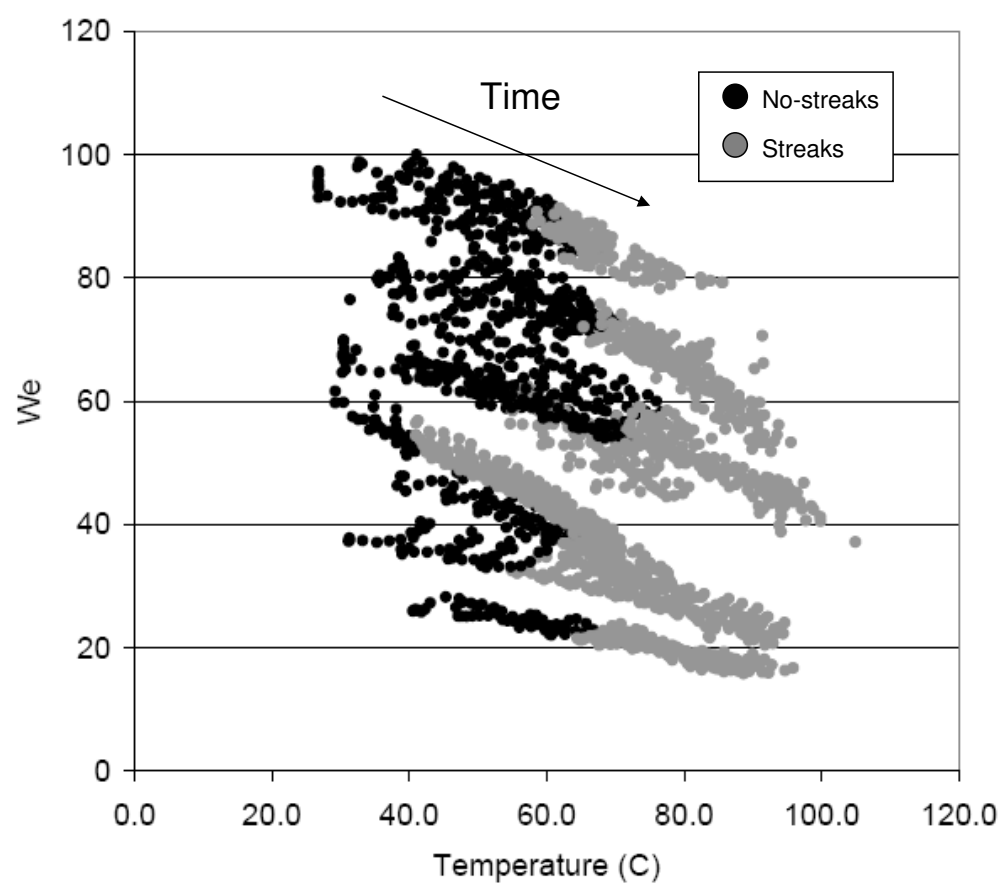

Figure 6.12: Plot of We vs Wheel Temperature showing how the onset of the streaking corresponds to the wheel temperature. Data from casts TBFA05-11,12,14, TBSU05-60,61,62,63,64,65, and TBSP06-12,13.

accounts for the wheel temperature. Since the appropriate dimensionless group to scale the wheel temperature by is not obvious, the dimensional temperature data will be used. Figure 6.12 shows $W e$ verses the wheel temperature and the onset of streaking. Figure 6.13 shows $P I$ verses the wheel temperature and the onset of streaking.

The data in Figures 6.12 and 6.13 show that the streaks seem to begin near a wheel temperature of 60 degrees $C$. The other boundaries on the operating window are still there. For example, the streaks also appear for $P I$ less than about 1.4, as reported by Byrne et al., 2006. When looking at the Figure 6.12 it appears that the streak begin at 40 degrees $C$ for one cast. However, in Figure 6.13, this is the cast at the lowest $P I$ and drops below a value of 1.4 at 40 degrees $C$. 


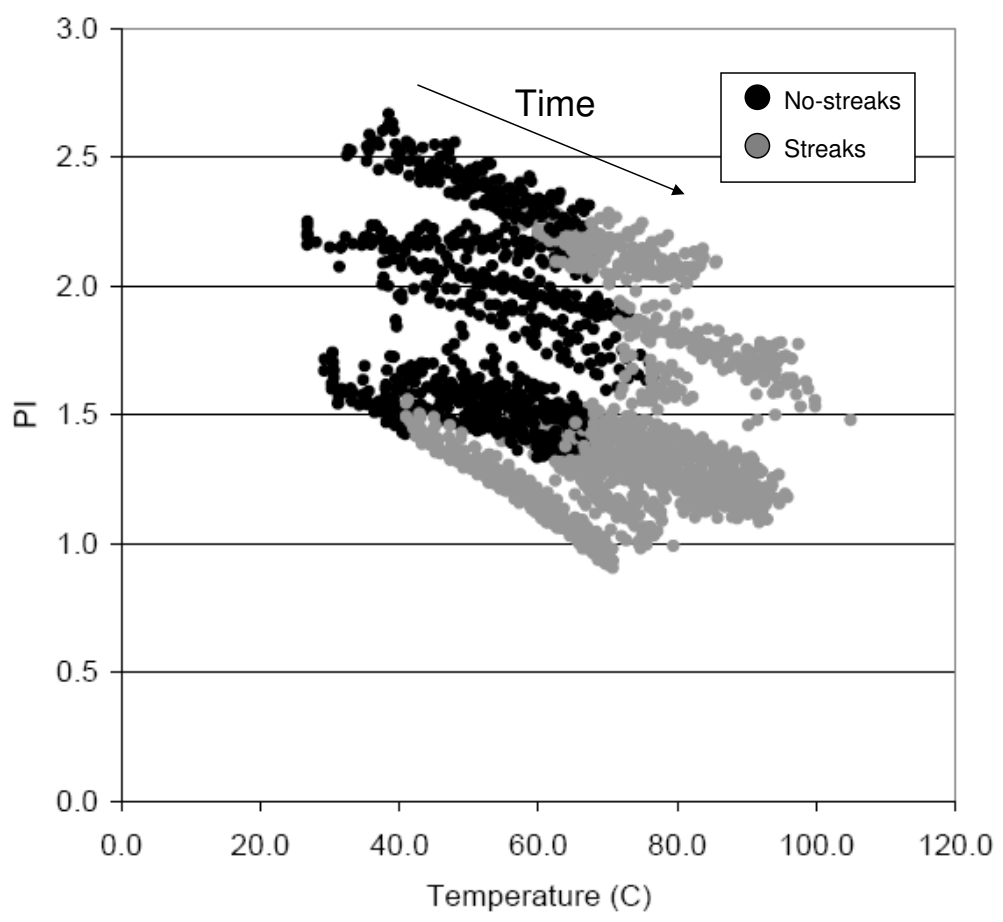

Figure 6.13: Plot of PI vs Wheel Temperature showing how the onset of the streaking corresponds to the wheel temperature. Data from casts TBFA05-11,12,14, TBSU05-60,61,62,63,64,65, and TBSP06-12,13. 




Figure 6.14: Through-cast measurements of T and L for cast TBSP06 20.

\subsection{Puddle length and thickness measurements}

The puddle length tends to increase throughout the cast, as discussed in Chapter 2. For only a few casts has $L$ been measured by the high speed video. The $T$ and $L$ measurements are shown in the following Figures. Initially $L$ decreases then begins to increases after about 1 or 2 seconds. It is not known why this turning occurs but it may be related to the streak formation discussed in Section 6.3. The two events seems to occur at the same time, but this may be coincidental. 


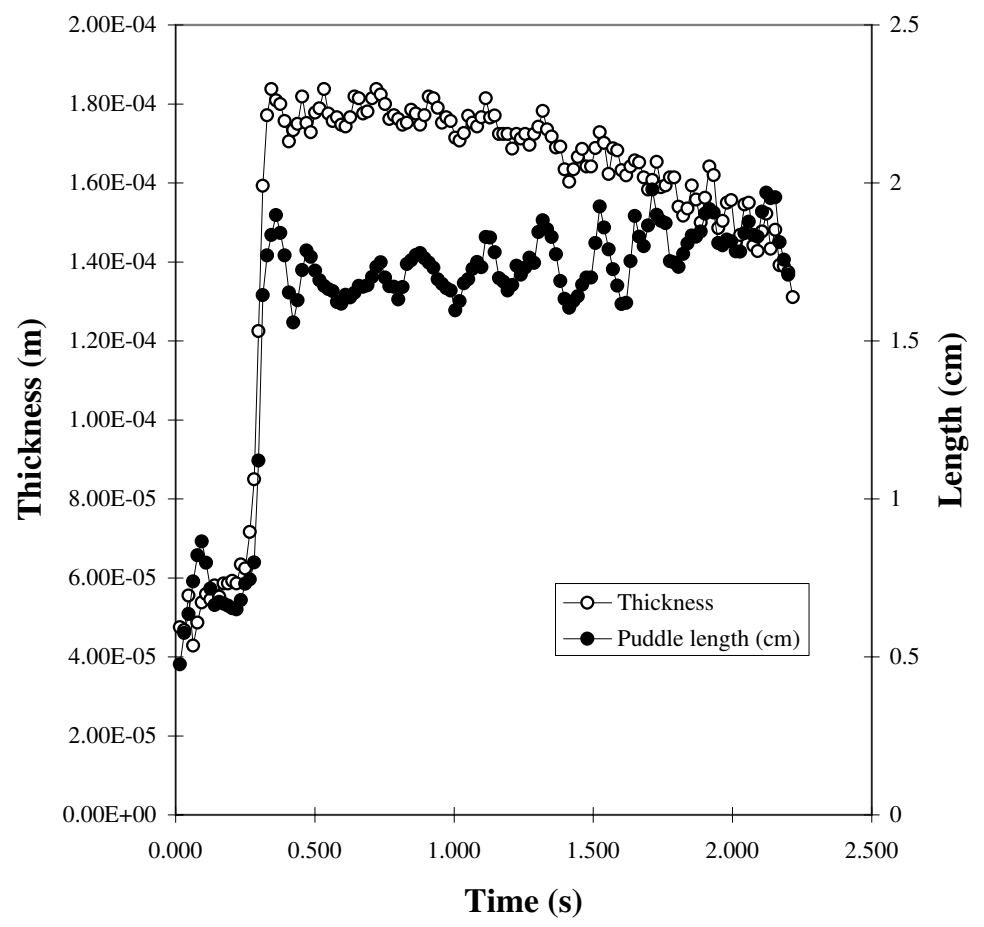

Figure 6.15: Through-cast measurements of T and L for cast TBSP06 65.

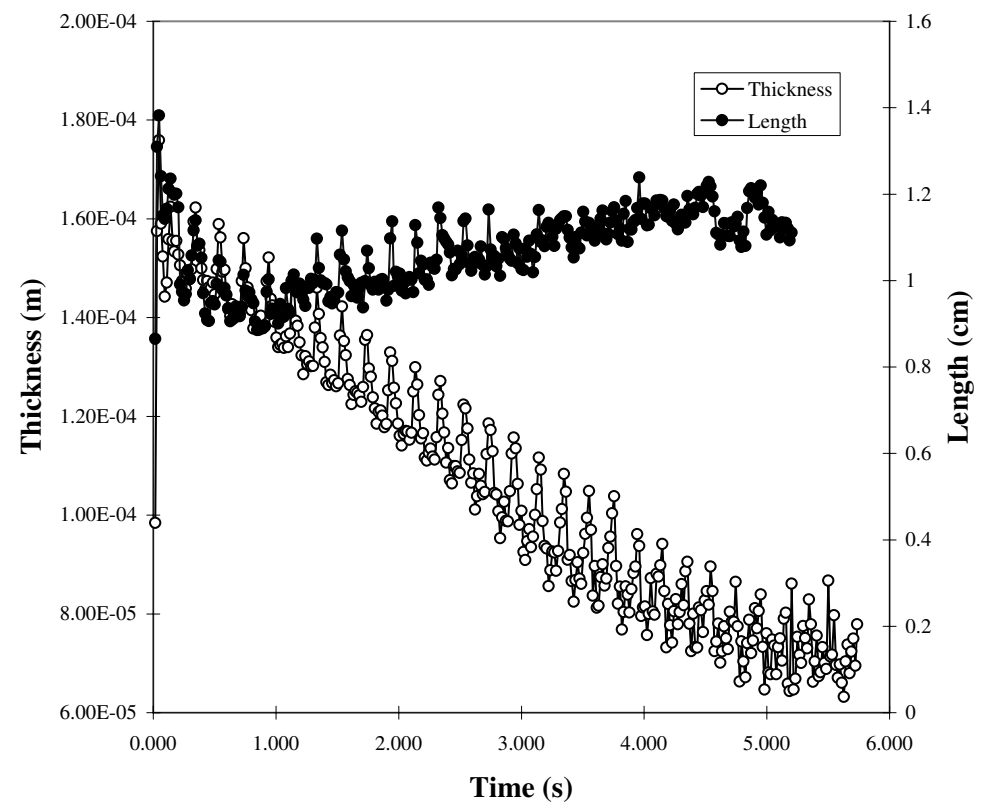

Figure 6.16: Through-cast measurements of T and L for cast ODSU06 22. 


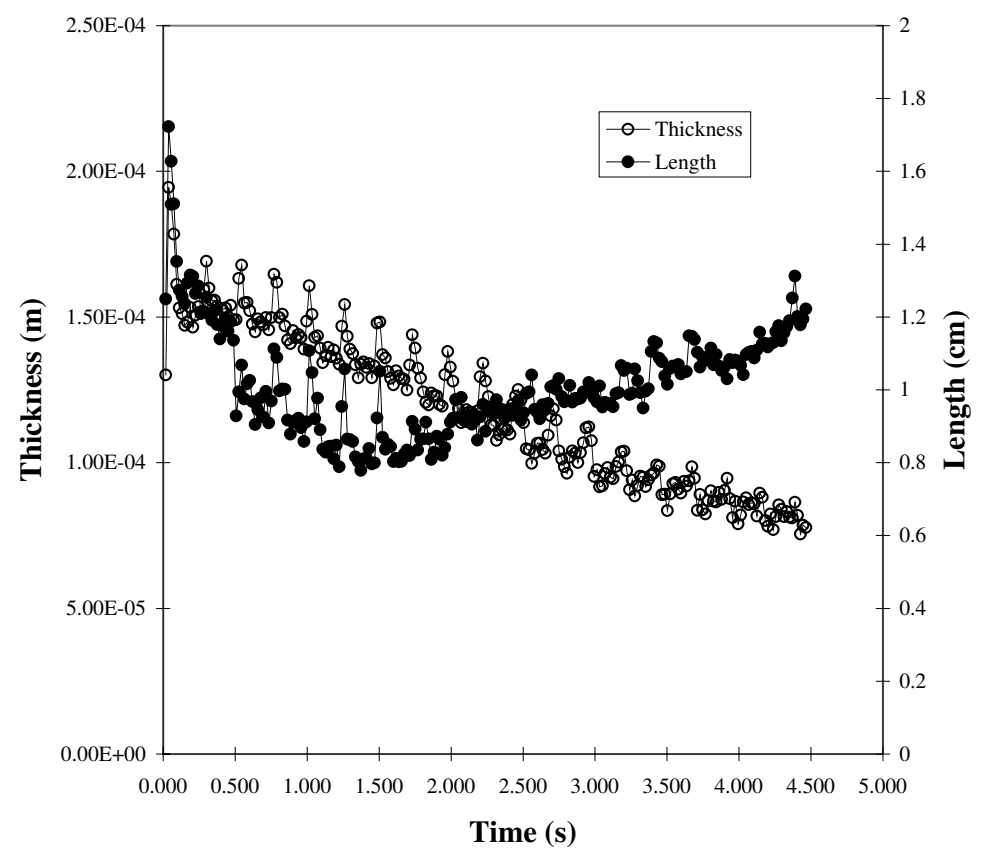

Figure 6.17: Through-cast measurements of T and L for cast ODSU06 40.



Figure 6.18: Through-cast measurements of T and L for cast ODSU06 42. 


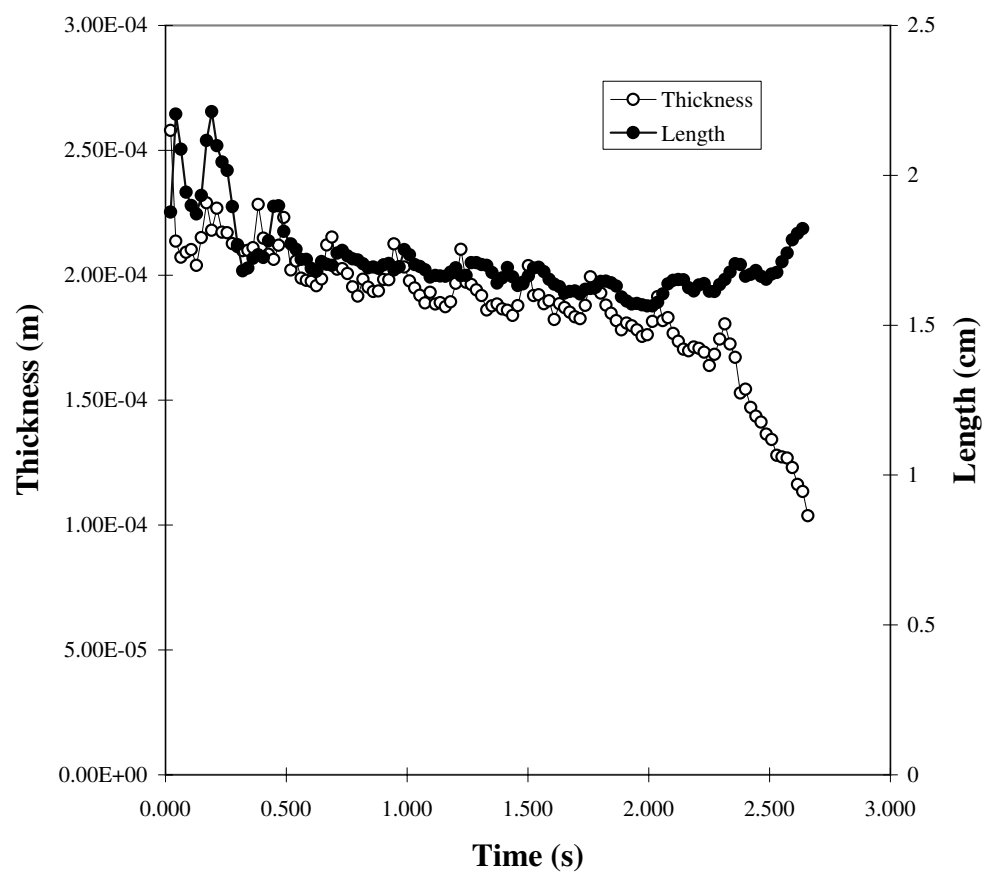

Figure 6.19: Through-cast measurements of T and L for cast ODSU06 43.

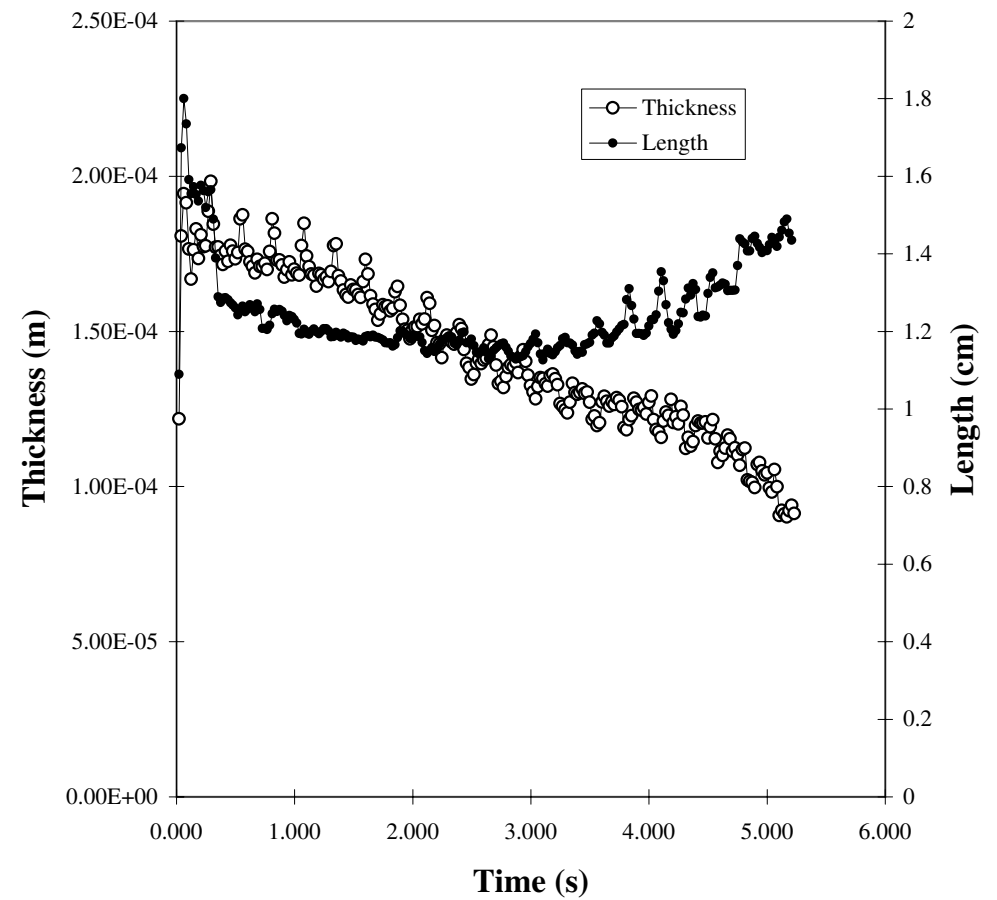

Figure 6.20: Through-cast measurements of T and L for cast ODSU06 47. 


\section{Appendix A}

\section{Dynamics of a Liquid Confined Between}

\section{Two Plates}

\section{A.1 Overview}

This Appendix presents a model of the dynamics of a $2 D$ section of liquid confined between two plates, as shown in Figure A.1. This model provides the motivation the subsequent work of $3 D$, axisymmetric droplets confined in a tube given in Chapter 4. The approach used here is to apply a Newtonian force balance to the center of mass of the liquid section. We will consider the capillary forces at the menisci to be the only forces acting on the center of mass. The system is in equilibrium when the curvature of each menisci is equal, as the pressures are the same. Perturbations to the menisci curvatures result in an imbalance of the forces acting on the center of mass. We apply a quasi-steady relation for the menisci shapes, allowing for them to deform provided they remain pieces of a circle. The center of mass of this system, which can be expressed geometrically, moves as the menisci vary in shape.

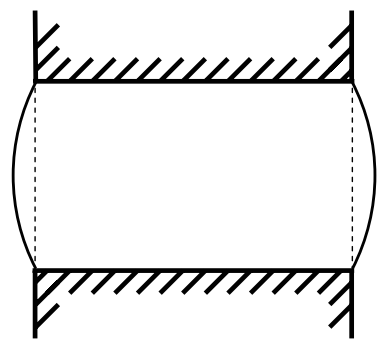

Figure A.1: Coupled droplet confined between two plates. 


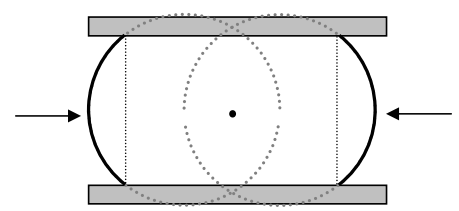

a)

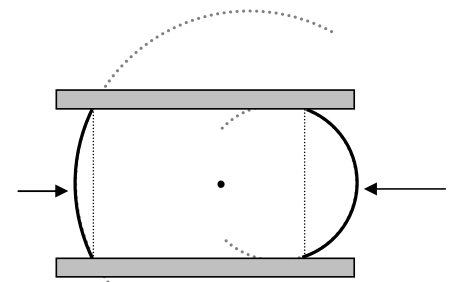

b)

Figure A.2: Schematic of 2D fluid confined between two parallel plates: a) Corresponds to an equilibrium configuration while b) corresponds to a disturbed state.



b)

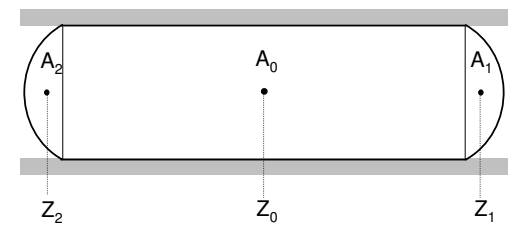

Figure A.3: Geometry of coupled droplet system confined between two plates. 
The approach we will use for this analysis is to define the center of mass in terms of the geometric parameters. Figure A.3 shows the geometry used for the analysis, where $R_{1}$ and $R_{2}$ are the radii of curvature and $\alpha$ and $\beta$ are the curvature angles. Any disturbance to the equilibrium geometry must conserve volume. We can expand the curvature angles in a power series around an equilibrium shape to give an analytic relation between curvature angles. The resulting expressions are used in the force balance to obtain the frequency at which the disturbance oscillates.

\section{A.2 Geometric formulation}

It is instructive to show the steps needed to calculate the center of mass of the liquid section. We can divide the entire liquid into smaller geometric sections, then sum the center of mass of each section $\left(Z_{c m i}\right)$ to yield the center of mass of the entire liquid $\left(Z_{c m}\right)$ by

$$
A Z_{c m}=\sum A_{i} Z_{c m i}
$$

Here $A$ is the entire area of the liquid and $A_{i}$ is the area of each section. Due to the symmetry of the system, it is convenient to divide the liquid into two areas on the right $\left(A_{1}\right)$ and the left $\left(A_{2}\right)$. Figure A.3 shows the geometry of these sections. Note that $R_{1} \sin (\alpha)=h=R_{2} \sin (\beta)$, therefore the system can be expressed in terms of variables $\alpha$ and $\beta$. The formulation used here is valid for $\alpha, \beta \in(0, \pi)$. However, the geometry of the parallel plates (shown in Figure A.3) restricts the menisci to sub-semicircular shapes, i.e. $\alpha, \beta \in\left(0, \frac{\pi}{2}\right)$. The areas of each section are given as 


$$
\begin{aligned}
& A_{0}=4 L h \\
& A_{1}=h^{2} \frac{2 \alpha-\sin (2 \alpha)}{2 \sin ^{2}(\alpha)} \\
& A_{2}=h^{2} \frac{2 \beta-\sin (2 \beta)}{2 \sin ^{2}(\beta)} .
\end{aligned}
$$

The center of mass for each section is given as

$$
\begin{aligned}
& Z_{0}=0 \\
& Z_{1}=L+\frac{4}{3} h\left(\frac{\sin ^{2} \alpha}{2 \alpha-\sin (2 \alpha)}-\frac{3}{4} \cos (\alpha)\right) \\
& Z_{2}=-\left[L+\frac{4}{3} h\left(\frac{\sin ^{2} \beta}{2 \beta-\sin (2 \beta)}-\frac{3}{4} \cos (\beta)\right)\right]
\end{aligned}
$$

where the coordinate system is placed at the center of mass of the undisturbed fluid.

When these expressions are placed into the center of mass calculations, we arrive at

$$
\begin{aligned}
A Z_{c m} & =\sum A_{i} Z_{c m i} \\
& =A_{1}\left(L+Z_{1}\right)-A_{2}\left(L+Z_{2}\right)
\end{aligned}
$$

Next we apply a force balance around the center of mass. The Young-Laplace equation gives the pressure jump across a curved menisci. Using these pressures as the forces acting on the center of mass gives the following dynamic equation

$$
\rho \frac{d^{2}}{d t^{2}} A Z_{c m}=\sigma(2 h)\left(\frac{1}{R_{2}}-\frac{1}{R_{1}}\right)
$$




$$
\frac{d^{2}}{d t^{2}}\left(A_{1} Z_{1}-A_{2} Z_{2}\right)+L \frac{d^{2}}{d t^{2}}\left(A_{1}-A_{2}\right)=\frac{2 \sigma}{\rho}(\sin (\beta)-\sin (\alpha))
$$

We want to solve this equation subject to the constant volume constraint,

$$
V=A_{1}+A_{2}+A_{0}=\text { const }
$$

which gives an implicit relation between $\alpha$ and $\beta$.

Finally, we can define $h$ as the characteristic length scales and $\left(\rho h^{3} / \sigma\right)^{1 / 2}$ as the characteristic time scale to nondimensionalize these equations, that is

$$
\begin{aligned}
\bar{L} & =\frac{L}{h} \\
\bar{t} & =\frac{t}{\left(\rho h^{3} / \sigma\right)^{1 / 2}} .
\end{aligned}
$$

All equations are in dimensionless form from here on and the overbars are dropped, unless otherwise noted.

\section{A.3 System dynamics}

The dimensionless system is expressed as

$$
\begin{aligned}
& \frac{d^{2}}{d t^{2}}\left[\left(\frac{2 \alpha-\sin (2 \alpha)}{2 \sin ^{2}(\alpha)}\right) \frac{4}{3}\left(\frac{\sin ^{2} \alpha}{2 \alpha-\sin (2 \alpha)}-\frac{3}{4} \cos (\alpha)\right)\right] \\
& -\frac{d^{2}}{d t^{2}}\left[\left(\frac{2 \beta-\sin (2 \beta)}{2 \sin ^{2}(\beta)}\right) \frac{4}{3}\left(\frac{\sin ^{2} \beta}{2 \beta-\sin (2 \beta)}-\frac{3}{4} \cos (\beta)\right)\right] \\
& +L \frac{d^{2}}{d t^{2}}\left[\frac{2 \alpha-\sin (2 \alpha)}{2 \sin ^{2}(\alpha)}-\frac{2 \beta-\sin (2 \beta)}{2 \sin ^{2}(\beta)}\right] \\
& =\sin (\beta)-\sin (\alpha)
\end{aligned}
$$

with the volume constraint 




Figure A.4: Disturbances of $\alpha$ and $\beta$ (around equilibrium value $\left(\alpha_{0}=\beta_{0}=\pi / 4\right.$ ) that satisfy constant volume constraint.

$$
4 L+\frac{2 \alpha-\sin (2 \alpha)}{2 \sin ^{2}(\alpha)}+\frac{2 \beta-\sin (2 \beta)}{2 \sin ^{2}(\beta)}=C .
$$

The volume constraint must be satisfied for all disturbances. Equation (A.15) can be solved iteratively around any equilibrium configuration to relate $\alpha$ in terms of $\beta$. For example, Figure A.4 shows the disturbances of $\alpha$ and $\beta$ (around equilibrium value $\left.\alpha_{0}=\beta_{0}=\pi / 4\right)$ that satisfy constant volume constraint.

\section{A.3.1 Analytic method}

This system of Equations (A.14) and (A.15) can be solved by a variety of methods. The approach that will be used here is to apply a regular perturbation and expand $\alpha$ and $\beta$ in a power series around an equilibrium state,

$$
\begin{aligned}
& \alpha(t)=\alpha_{0}+\epsilon \alpha_{1}(t)+\epsilon^{2} \alpha_{2}(t)+\epsilon^{3} \alpha_{3}(t)+\ldots \\
& \beta(t)=\beta_{0}+\epsilon \beta_{1}(t)+\epsilon^{2} \beta_{2}(t)+\epsilon^{3} \beta_{3}(t)+\ldots
\end{aligned}
$$


Here we can choose any equilibrium state where $\alpha_{0}=\beta_{0}$. Note that this is the one equilibrium value for $\alpha, \beta \in\left(0, \frac{\pi}{2}\right)$. Substituting Equations (A.16) and (A.17) into Equation (A.15) and solving at orders of $\epsilon$ one finds,

$$
\begin{aligned}
& C=\frac{2 \alpha_{0}-\sin \left(2 \alpha_{0}\right)}{\sin ^{2}\left(\alpha_{0}\right)}+4 L \\
& \beta_{1}=-\alpha_{1} \\
& \beta_{2}=-\alpha_{2}+\frac{4 \alpha_{0}+\alpha_{0} \cos \left(2 \alpha_{0}\right)-3 \sin \left(2 \alpha_{0}\right)}{\alpha_{0} \sin \left(2 \alpha_{0}\right)+\cos \left(2 \alpha_{0}\right)-1} \\
& \beta_{3}=-\alpha_{3}+f\left(\alpha_{0}, \alpha 1(t), \alpha 2(t)\right)
\end{aligned}
$$

From this expansion it is observed that at $\mathrm{O}\left(\epsilon^{0}\right)$ the initial conditions, $\left(\alpha_{0}=\beta_{0}\right)$, determine the constant $C$. At $\mathrm{O}\left(\epsilon^{1}\right)$ there is a linear relation between $\alpha$ and $\beta$. It is necessary to go to higher orders of $\epsilon$ before nonlinearities begin to appear. Substituting Equations (A.18)-(A.21) into Equation (A.14), the first orders of $\epsilon$ give

$$
\begin{array}{lr}
O\left(\epsilon^{0}\right): \quad 0 & =0 \\
O\left(\epsilon^{1}\right): \frac{d^{2} \alpha_{1}}{d t^{2}}+\lambda^{2} \alpha_{1} & =0
\end{array}
$$

where

$$
\lambda^{2}=\frac{1}{8}\left[\frac{-2 \cos \left(\alpha_{0}\right)+3 \cos \left(3 \alpha_{0}\right)-\cos \left(5 \alpha_{0}\right)}{-2 L-4 \alpha_{0}+\left(3+2 L \alpha_{0}\right) \sin \left(2 \alpha_{0}\right)+\left(2 L-2 \alpha_{0}\right) \cos \left(2 \alpha_{0}\right)}\right]
$$

Equation (A.23) is that of a harmonic oscillator and has the solution

$$
\alpha_{1}(t)=A \cos (\lambda t)
$$


This satisfies the initial condition $\alpha(0)=\alpha_{0}+\epsilon A$. Here $A$ is the amplitude of the motion and the phase is initially set. That is, since we are looking for periodic solutions we can set the initial conditions to any point in the phase plane (due to the autonomous nature of the system).

At the lowest order, the frequency at which the menisci oscillate is given by $\lambda$. There are three limiting cases in Equation (A.24):

i) $\lambda^{2} \rightarrow 0$ as $\alpha_{0} \rightarrow \pi / 2$. The case $\alpha_{0}=\pi / 2$ corresponds to the point where the eigenvalues change from being a complex conjugate pair with zero real part to a double eigenvalue of zero. For values of $\alpha_{0}>\pi / 2$, there is one positive and one negative eigenvalue indicating instability. (Note that $\alpha_{0}>\pi / 2$ shapes are only allowed when the fluid in pinned at the edge of the parallel plates, as shown in Figure A.1.)

ii) $\lambda^{2} \rightarrow 12 / L$ as $\alpha_{0} \rightarrow 0$. Equation (A.24) is indeterminant for $\alpha_{0}=0$ and l'Hospital's rule is applied four times to find the limit.

iii) $\lambda^{2} \rightarrow 0$ as $L \rightarrow \infty$.

Figure A.5 plots $\lambda$ vs $\alpha_{0}$ for various values of $L$ (note that $\alpha_{0}$ ranges from 0.001 to $\pi / 2)$.

\section{A.3.2 Higher order corrections}

To obtain the lowest order solution, we expanded the curvature angles using a regular perturbation scheme. However, when we attempt to carry out the procedure to higher orders, resonance terms appear in the solution, which cause problems 


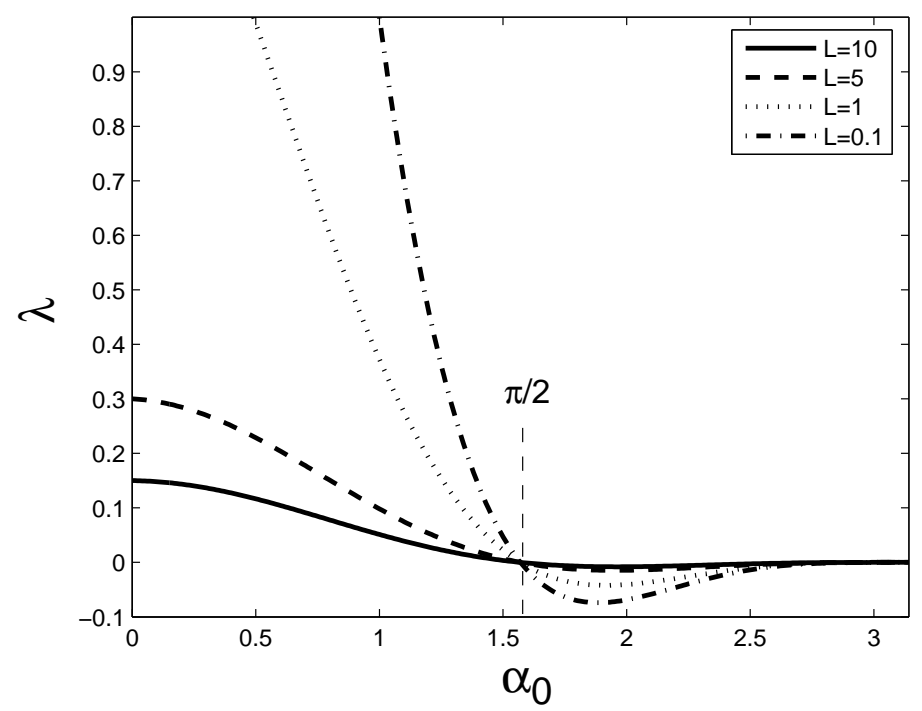

Figure A.5: Vibration frequency vs equilibrium angle for various aspect ratios.

at long times. Therefore, we must use a special perturbation technique to remove these resonance terms. Lindstedt's method is used to stretch time and build a period-amplitude relation in the solution, thereby removing the resonant terms.

Before applying this procedure, let us scale time by the frequency, $\lambda$. At this stage $\lambda$ is arbitrary (but will end up being the same as given in Equation (A.24)). That is,

$$
t=\frac{t^{*}}{\lambda}
$$

Now we stretch this new time by

$$
t^{*}=\omega \tau
$$

The force balance now becomes 


$$
\omega^{2} \lambda^{2} \frac{d^{2}}{d \tau^{2}}\left(A_{1} Z_{1}-A_{2} Z_{2}\right)+\omega^{2} \lambda^{2} L \frac{d^{2}}{d \tau^{2}}\left(A_{1}-A_{2}\right)=\sin (\beta)-\sin (\alpha)
$$

Now we expand as

$$
\begin{aligned}
\alpha(\tau) & =\alpha_{0}+\epsilon \alpha_{1}(\tau)+\epsilon^{2} \alpha_{2}(\tau)+\ldots \\
\beta(\tau) & =\alpha_{0}+\epsilon \beta_{1}(\tau)+\epsilon^{2} \beta_{2}(\tau)+\ldots \\
\omega & =1+k_{1} \epsilon+k_{2} \epsilon^{2}+\ldots
\end{aligned}
$$

Now we go through the same procedure of expanding the volume constraint to relate $\alpha$ and $\beta$ at each order. Then plug the results into the force balance to give

$$
\begin{aligned}
& O\left(\epsilon^{1}\right): \frac{d^{2} \alpha_{1}}{d \tau^{2}}+\alpha_{1}=0 \\
& O\left(\epsilon^{2}\right): \frac{d^{2} \alpha_{2}}{d \tau^{2}}+\alpha_{2}=-2 k_{1} \frac{d^{2} \alpha_{1}}{d \tau^{2}}-F_{1}\left[\frac{\alpha_{1}^{2}}{2}+\alpha_{1} \frac{d^{2} \alpha_{1}}{d \tau^{2}}+\left(\frac{d \alpha_{1}}{d \tau}\right)^{2}\right] \\
& O\left(\epsilon^{3}\right): \frac{d^{2} \alpha_{3}}{d \tau^{2}}+\alpha_{3}=f\left(\alpha_{0}, \alpha_{1}, \alpha_{2}\right)
\end{aligned}
$$

where

$$
F_{1}=\left(\frac{2 \alpha_{0} \cos \left(\alpha_{0}\right)^{2}-3 \sin \left(\alpha_{0}\right) \cos \left(\alpha_{0}\right)+\alpha_{0}}{1-\cos \left(\alpha_{0}\right)^{2}-\alpha_{0} \sin \left(\alpha_{0}\right) \cos \left(\alpha_{0}\right)}\right)
$$

The $O\left(\epsilon^{1}\right)$ equation has the general solution

$$
\alpha_{1}(\tau)=A \cos (\tau+\phi)
$$

where $\phi$ is a phase shift. Again, since we are only looking for periodic solutions and the system is autonomous, $\phi$ is set to zero. Equation (A.36) satisfies the initial 
condition $\alpha(0)=\alpha_{0}+\epsilon A$. Substituting Equation (A.36) into Equation (A.33), one finds

$$
\frac{d^{2} \alpha_{2}}{d \tau^{2}}+\alpha_{2}=-2 k_{1} A \cos (\tau)-F_{1} A^{2}\left[-\frac{3}{4} \cos (2 \tau)+\frac{1}{4}\right] .
$$

Here $k_{1}$ must be zero to remove resonant terms and the period-amplitude correction does not appear at this order. We can solve for $\alpha_{2}(\tau)$ and apply the initial condition to give

$$
\alpha_{2}(\tau)=\frac{F_{1} A^{2}}{2} \cos (\tau)+C \sin (\tau)-\frac{F_{1} A^{2}}{4} \cos (2 \tau)-\frac{F_{1} A^{2}}{4}
$$

Now we can substitute Equation (A.38) into the $O\left(\epsilon^{3}\right)$ problem. Fortunately, we do not need the full solution for $\alpha_{3}(\tau)$, rather we only need to identify the coefficients of the resonant terms. That is, Equation (A.34) has the form

$$
\frac{d^{2} \alpha_{3}}{d \tau^{2}}+\alpha_{3}=F_{2} \cos (\tau)+F_{3} \sin (\tau)+N \cdot R \cdot T
$$

It turns out that the $F_{3}$ term is zero. Removing the resonant term from the $F_{2}$ equation gives the desired relation between $k_{2}$ and $A$. For arbitrary values of $\alpha_{0}$, this expression is rather cumbersome. However, for the special case of $\alpha_{0}=\pi / 4$ we find,

$$
k_{2}=\frac{A^{2}}{16}\left[\frac{15 L \pi^{2}-30 \pi^{2}+130 \pi-52 L \pi-112+16 L}{-24+16 L-8 L \pi+L \pi^{2}-2 \pi^{2}+14 \pi}\right] .
$$

Figure A.6 plots $\omega$ verses the disturbance amplitude as the aspect ratio $L$ is varied. Here $A^{*}=\epsilon A$ and recall that $\omega=1+\epsilon^{2} k_{2}+O\left(\epsilon^{3}\right)$. There is little influence of $L$ on the bending observed in Figure A.6. 


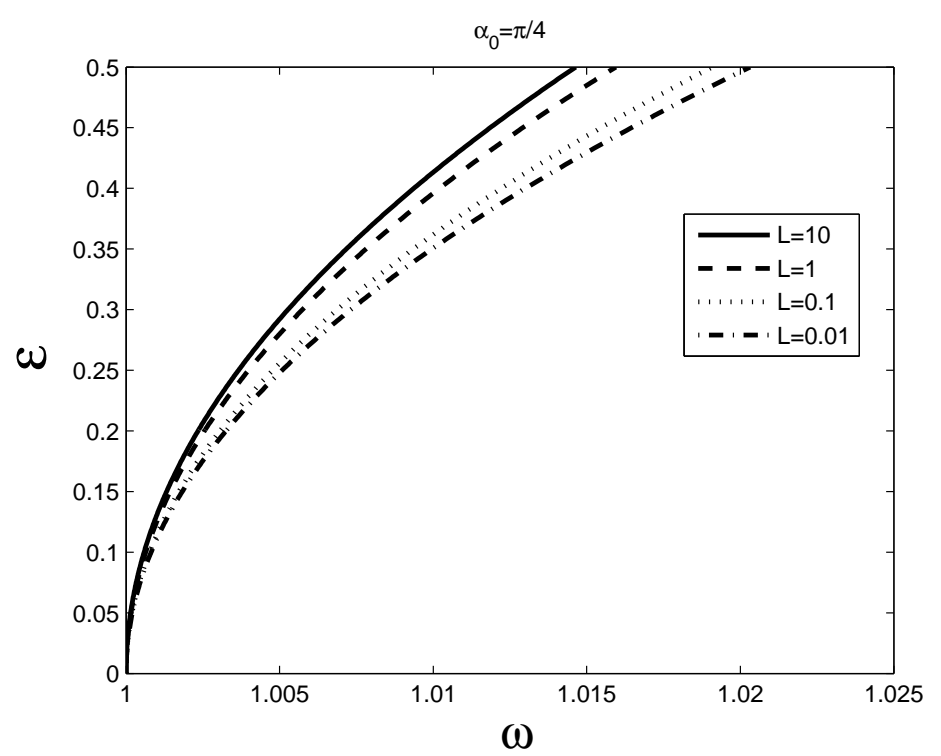

Figure A.6: Frequency-amplitude relation for $\alpha_{0}=\pi / 4$ as the aspect ratio $L$ is varied.

The next issue is whether the frequency-amplitude relation varies with $\alpha_{0}$. Figure A.7 plots $\omega$ verses the disturbance amplitude as $\alpha_{0}$ is varied and $L$ is fixed to 10 . It turns out that the bending observed varies significantly (even changes direction) as $\alpha_{0}$ changes. This is qualitatively similar to the results presented in Figure 4.4 b). Section 4.6 provides a further discussion of this bending behavior. Varying $L$ has little influence on the results shown in Figure A.7.

\section{A.4 Comparison to cross-wave defect frequency}

It is of interest to compare the frequency of vibration of the liquid with the frequency of the cross-stream defect in the Melt Spinning process. Recall that the cross-stream defect frequency scales as 


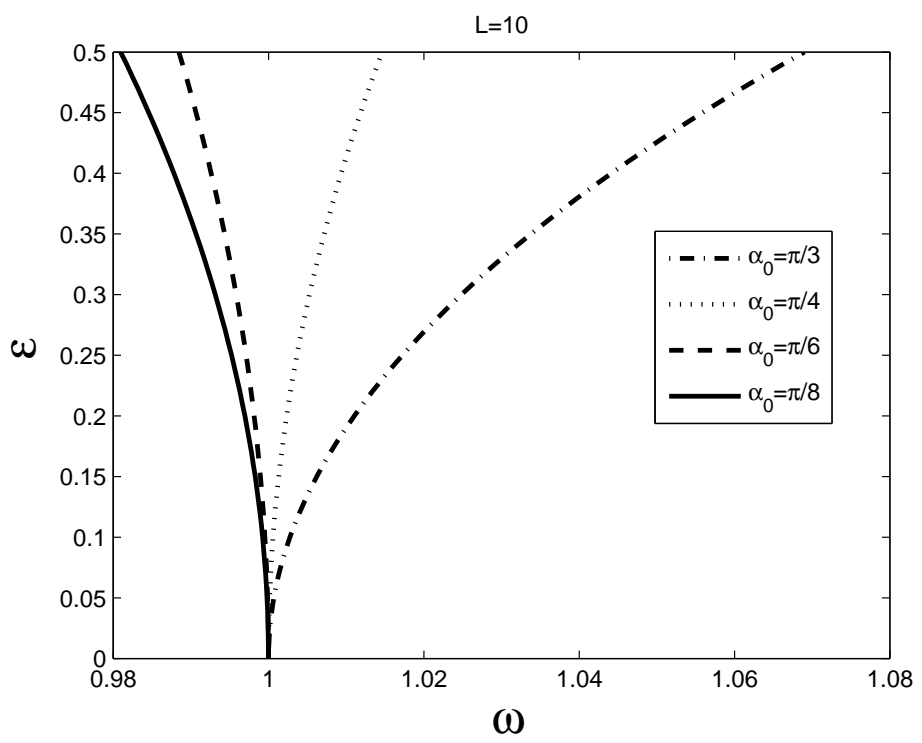

Figure A.7: Frequency-amplitude relation as $\alpha_{0}$ is varied and the aspect ratio is fixed at $L=10$.

$$
\nu=1.95\left(\frac{\sigma}{\rho G^{3}}\right)^{1 / 2} .
$$

where $\nu$ is the linear vibration frequency. We are interested in comparing the constant 1.95 to that predicted by the coupled droplet model. To compare this directly to the frequency of vibration of the liquid section, we must redimensionalize the variables and convert $\lambda$ to a linear frequency. After converting $G=2 h$ and $L_{p}=2 L\left(G\right.$ is the gap height and $L_{p}$ is the puddle length in the melt spinning process), we arrive at

$$
f=\frac{2 \lambda}{\pi}\left(\frac{\sigma}{\rho G^{3}}\right)^{1 / 2},
$$

where $f$ is the linear frequency. Recall that $\lambda$ depends upon the aspect ratio of the liquid section and the curvature angle $\alpha_{0}$. Figure A.8 shows how $\frac{2 \lambda}{\pi}$ varies with $\frac{L_{p}}{G}$. For typical values of $\frac{L_{p}}{G}$ the vibration frequency is much slower than that observed 




Figure A.8: Vibration constant as the cast puddle aspect ratio is varied, for various curvature angles.

for the cross-stream wave defect. This result indicates that the center-of-mass oscillation that is predicted for cylindrical-cap menisci shapes does not correspond to the cross-stream wave defect. The predicted vibration frequency scales inversely with the area of the $2 D$ section of fluid, whereas the cross-stream wave motion is independent of the fluid area (or length of the molten metal puddle). The model problem given in Section 3.7, where the menisci can deform freely, provides a closer approximation to the cross-stream wave frequency. 


\section{Appendix B}

\section{Experimental Data}

This appendix is a summary of data from experiments carried out from Fall 2001 until Fall 2006. A more complete record of these casts is available electronically.

Each cast is identified by a unique label, indicating primary operators, season, year and cast number. In Summer 2005, casts are labeled as TB, though were predominantly OD casts. An indicator is used beside the cast ID when the gap distance or puddle length have been sought through that cast. Many high-speed videos are available from which further puddle length data could be extracted. Also indicated is when the nozzle has been modified or the wheel heated. Nozzle modification refers to shaping of the nozzle, increasing the breadth of the nozzle slot, or changes in the slot width.

Average values of thickness, wheel speed and pressure are reported. The initial mass and initial (or 'hot') gap distance are shown. In cases where no thickness data is present, no pressure measurement is available because the hydrostatic head could not be calculated.

Table B.1: Operating conditions and measured ribbon thickness from casting experiments Summer 2006 - Spring 2002.

\begin{tabular}{lccccccc} 
Cast ID & $\mathrm{T}$ & $\mathrm{G}$ & $\mathrm{U}$ & $\mathrm{P}$ & Mass & & \\
\hline ODSU06-01 ${ }^{\dagger \ddagger}$ & $(\mathrm{mm})$ & $(\mathrm{mm})$ & $(\mathrm{m} / \mathrm{s})$ & $(\mathrm{Pa})$ & $(\mathrm{g})$ & Alloy & Substrate \\
ODSU06-02 ${ }^{\dagger \ddagger}$ & 0.163 & 0.914 & 9.4 & - & 451 & $7 \% \mathrm{Si}$ & Small CuBe \\
ODSU06-03 ${ }^{\dagger \ddagger}$ & 0.139 & 0.787 & 8.3 & 3992 & 565 & $7 \% \mathrm{Si}$ & Small CuBe \\
${ }^{\dagger}=$ Gap measure, ${ }^{\ddagger}=$ Puddle length, ${ }^{\star}=$ modified nozzle, ${ }^{\diamond}=$ heated wheel
\end{tabular}


Table B.1: (Continued)

\begin{tabular}{|c|c|c|c|c|c|c|c|}
\hline Cast ID & $\begin{array}{c}\mathrm{T} \\
(\mathrm{mm})\end{array}$ & $\begin{array}{c}\mathrm{G} \\
(\mathrm{mm})\end{array}$ & $\begin{array}{c}\mathrm{U} \\
(\mathrm{m} / \mathrm{s})\end{array}$ & $\begin{array}{c}\mathrm{P} \\
(\mathrm{Pa})\end{array}$ & $\begin{array}{c}\text { Mass } \\
(\mathrm{g})\end{array}$ & Alloy & Substrate \\
\hline ODSU06-04 ${ }^{\dagger \ddagger}$ & 0.105 & 1.041 & 9.5 & 2869 & 441 & $7 \% \mathrm{Si}$ & Small $\mathrm{CuBe}$ \\
\hline ODSU06-05 * & - & 1.041 & 9.5 & - & 518 & $7 \% \mathrm{Si}$ & Small CuBe \\
\hline ODSU06-06 ${ }^{\dagger}$ & 0.252 & 0.787 & 6.4 & 5820 & 400 & $7 \% \mathrm{Si}$ & Small $\mathrm{CuBe}$ \\
\hline ODSU06-07 †夫 & - & 0.787 & 4.8 & - & 577 & $7 \% \mathrm{Si}$ & Small CuBe \\
\hline ODSU06-08 ${ }^{\dagger \star}$ & - & 0.787 & 4.8 & - & 527 & $7 \% \mathrm{Si}$ & Small CuBe \\
\hline ODSU06-09 ${ }^{\dagger}$ & 0.119 & 0.508 & 7.6 & 7347 & 1020 & $7 \% \mathrm{Si}$ & Small CuBe \\
\hline ODSU06-10 ${ }^{\dagger}$ & 0.134 & 0.508 & 9.5 & 6765 & 914 & $7 \% \mathrm{Si}$ & Small CuBe \\
\hline ODSU06-11 ${ }^{\dagger}$ & 0.250 & 0.787 & 6.4 & 4963 & 676 & $7 \% \mathrm{Si}$ & Small CuBe \\
\hline ODSU06-12 ${ }^{\dagger}$ & 0.157 & 0.787 & 6.4 & 3357 & 618 & $7 \% \mathrm{Si}$ & Small CuBe \\
\hline ODSU06-13 & - & 0.787 & 9.5 & - & 632 & $7 \% \mathrm{Si}$ & Small CuBe \\
\hline ODSU06-14 & 0.090 & 0.787 & 9.5 & 3102 & 627 & $7 \% \mathrm{Si}$ & Small CuBe \\
\hline ODSU06-15 ${ }^{\dagger}$ & - & 0.610 & 5.8 & - & 872 & $7 \% \mathrm{Si}$ & Small CuBe \\
\hline ODSU06-16 †‡ & 0.084 & 0.787 & 11.2 & 3325 & 646 & $7 \% \mathrm{Si}$ & Small CuBe \\
\hline ODSU06-17 †‡ & 0.071 & 0.787 & 12.8 & 3170 & 637 & $7 \% \mathrm{Si}$ & Small CuBe \\
\hline ODSU06-18 ${ }^{\dagger \diamond}$ & 0.176 & 0.610 & 5.7 & 4979 & 683 & $7 \% \mathrm{Si}$ & Small CuBe \\
\hline ODSU06-19 ${ }^{\dagger}$ & 0.068 & 0.508 & 9.1 & 3031 & 946 & $7 \% \mathrm{Si}$ & Small CuBe \\
\hline ODSU06-20 ${ }^{\dagger}$ & 0.061 & 0.508 & 9.1 & 5019 & 973 & $7 \% \mathrm{Si}$ & Small CuBe \\
\hline ODSU06-21 ${ }^{\dagger}$ & 0.119 & 0.610 & 9.5 & 6015 & 835 & $7 \% \mathrm{Si}$ & Small $\mathrm{CuBe}$ \\
\hline ODSU06-22 ${ }^{\dagger}$ & 0.107 & 0.610 & 9.5 & 5142 & 1319 & & Small CuBe \\
\hline ODSU06-23 ${ }^{\dagger}$ & 0.117 & 0.711 & 9.5 & 5489 & 1413 & $7 \% \mathrm{Si}$ & Small CuBe \\
\hline ODSU06-24 ${ }^{\dagger}$ & 0.147 & 1.016 & 9.5 & 4593 & 1460 & $7 \% \mathrm{Si}$ & Small CuBe \\
\hline ODSU06-25 ${ }^{\dagger}$ & 0.149 & 0.610 & 6.0 & 5427 & 1456 & $7 \% \mathrm{Si}$ & Small CuBe \\
\hline ODSU06-26 ${ }^{\dagger}$ & 0.120 & 0.610 & 9.5 & 5624 & 1319 & $7 \% \mathrm{Si}$ & Small CuBe \\
\hline ODSU06-27 ${ }^{\dagger}$ & 0.208 & 0.610 & 6.4 & 6002 & 593 & $7 \% \mathrm{Si}$ & Small CuBe \\
\hline ODSU06-28 ${ }^{\dagger}$ & - & 0.610 & 6.4 & - & 820 & $7 \% \mathrm{Si}$ & Small CuBe \\
\hline ODSU06-29 ${ }^{\dagger}$ & - & 0.787 & 6.21 & - & 571 & $7 \% \mathrm{Si}$ & Small CuBe \\
\hline ODSU06-30 ${ }^{\dagger}$ & 0.162 & 0.787 & 6.4 & 3703 & 582 & $7 \% \mathrm{Si}$ & Small CuBe \\
\hline ODSU06-31 ${ }^{\dagger}$ & 0.164 & 0.787 & 6.4 & 3597 & 569 & $7 \% \mathrm{Si}$ & Small CuBe \\
\hline ODSU06-32 ${ }^{\dagger}$ & 0.095 & 0.610 & 9.4 & 5685 & 1323 & $7 \% \mathrm{Si}$ & Small CuBe \\
\hline ODSU06-33 ${ }^{\dagger}$ & 0.135 & 0.610 & 9.6 & 5232 & 1329 & $7 \% \mathrm{Si}$ & Small CuBe \\
\hline ODSU06-34 ${ }^{\dagger}$ & - & 0.787 & - & - & 563 & $7 \% \mathrm{Si}$ & Small CuBe \\
\hline ODSU06-35 ${ }^{\dagger}$ & - & 0.787 & - & - & 532 & $7 \% \mathrm{Si}$ & Small CuBe \\
\hline ODSU06-36 ${ }^{\dagger}$ & 0.115 & 0.381 & 5.2 & 6661 & 603 & $7 \% \mathrm{Si}$ & Small CuBe \\
\hline ODSU06-37 † & 0.197 & 0.508 & 5.1 & 7207 & 861 & $7 \% \mathrm{Si}$ & Small CuBe \\
\hline ODSU06-38 ${ }^{\dagger}$ & - & 0.762 & - & - & 556 & $7 \% \mathrm{Si}$ & Small CuBe \\
\hline ODSU06-39 ${ }^{\dagger}$ & 0.136 & 0.559 & 7.95 & 5963 & 586 & $7 \% \mathrm{Si}$ & Small CuBe \\
\hline TBSP06-01 & - & 0.635 & 12.3 & - & 300 & $7 \% \mathrm{Si}$ & Large $\mathrm{CuBe}$ \\
\hline TBSP06-02 & - & 0.635 & 8.2 & - & 300 & $7 \% \mathrm{Si}$ & Large $\mathrm{CuBe}$ \\
\hline TBSP06-03 & 0.072 & 0.787 & 8.9 & - & 798 & $7 \% \mathrm{Si}$ & Large $\mathrm{CuBe}$ \\
\hline TBSP06-04 & 0.239 & 1.092 & 8.6 & 3958 & 505 & $7 \% \mathrm{Si}$ & Large $\mathrm{CuBe}$ \\
\hline TBSP06-05 & 0.106 & 1.092 & 8.6 & 1528 & 277 & $7 \% \mathrm{Si}$ & Large $\mathrm{CuBe}$ \\
\hline
\end{tabular}

${ }^{\dagger}=$ Gap measure, ${ }^{\ddagger}=$ Puddle length, ${ }^{\star}=$ modified nozzle, ${ }^{\diamond}=$ heated wheel 
Table B.1: (Continued)

\begin{tabular}{|c|c|c|c|c|c|c|c|}
\hline Cast ID & $\begin{array}{c}\mathrm{T} \\
(\mathrm{mm})\end{array}$ & $\begin{array}{c}\mathrm{G} \\
(\mathrm{mm})\end{array}$ & $\begin{array}{c}\mathrm{U} \\
(\mathrm{m} / \mathrm{s})\end{array}$ & $\begin{array}{c}\mathrm{P} \\
(\mathrm{Pa})\end{array}$ & $\begin{array}{c}\text { Mass } \\
(\mathrm{g})\end{array}$ & Alloy & Substrate \\
\hline TBSP06-06 & 0.091 & 1.092 & 8.6 & 1711 & 265 & $7 \% \mathrm{Si}$ & Large $\mathrm{CuBe}$ \\
\hline TBSP06-07 & 0.063 & 0.787 & 9.6 & 1657 & - & $7 \% \mathrm{Si}$ & Large $\mathrm{CuBe}$ \\
\hline TBSP06-08 & 0.087 & 0.787 & 8.9 & 1803 & 522 & $7 \% \mathrm{Si}$ & Large $\mathrm{CuBe}$ \\
\hline TBSP06-09 & - & 0.787 & 8.4 & - & 502 & $7 \% \mathrm{Si}$ & Large $\mathrm{CuBe}$ \\
\hline TBSP06-10 & - & 1.092 & 7.8 & - & 504 & $7 \% \mathrm{Si}$ & Large $\mathrm{CuBe}$ \\
\hline TBSP06-11 & - & 0.787 & 5.7 & - & 741 & $7 \% \mathrm{Si}$ & Large $\mathrm{CuBe}$ \\
\hline TBSP06-12 ${ }^{\dagger}$ & 0.175 & 0.610 & 5.7 & 4849 & 880 & $7 \% \mathrm{Si}$ & Small CuBe \\
\hline TBSP06-13 ${ }^{\dagger}$ & 0.121 & 0.610 & 9.9 & 4818 & 887 & $7 \% \mathrm{Si}$ & Small CuBe \\
\hline TBSP06-14 ${ }^{\dagger}$ & 0.225 & 0.508 & 5.7 & 8362 & 744 & $7 \% \mathrm{Si}$ & Small CuBe \\
\hline TBSP06-15 ${ }^{\dagger}$ & 0.164 & 0.508 & 7.6 & 8898 & 980 & $7 \% \mathrm{Si}$ & Small CuBe \\
\hline TBSP06-16 ${ }^{\dagger}$ & 0.165 & 0.508 & 5.8 & 6774 & 865 & $7 \% \mathrm{Si}$ & Small CuBe \\
\hline TBSP06-17 ${ }^{\dagger}$ & 0.176 & 1.016 & 7.6 & 3307 & 860 & $7 \% \mathrm{Si}$ & Small CuBe \\
\hline TBSP06-18 & 0.175 & 1.016 & 5.8 & 2670 & 888 & $7 \% \mathrm{Si}$ & Small CuBe \\
\hline TBSP06-19 ${ }^{\dagger}$ & 0.109 & 0.508 & 9.5 & 6432 & 868 & $7 \% \mathrm{Si}$ & Small CuBe \\
\hline TBSP06-20 ${ }^{\dagger}$ & 0.155 & 0.508 & 4.4 & 7385 & 950 & $7 \% \mathrm{Si}$ & Small CuBe \\
\hline TBSP06-21 ${ }^{\dagger}$ & - & 1.067 & 7.8 & - & 333 & $7 \% \mathrm{Si}$ & Small CuBe \\
\hline TBSP06-22 & - & 0.914 & 9.4 & - & 430 & $7 \% \mathrm{Si}$ & Small $\mathrm{CuBe}$ \\
\hline TBSP06-23 & - & 0.914 & 9.4 & - & 470 & $7 \% \mathrm{Si}$ & Small CuBe \\
\hline TBSP06-24 * & - & 0.914 & 9.4 & - & 536 & $7 \% \mathrm{Si}$ & Small CuBe \\
\hline TBFA05-01 & 0.121 & 1.041 & 9.6 & 4032 & 979 & $7 \% \mathrm{Si}$ & Large $\mathrm{CuBe}$ \\
\hline TBFA05-02 & 0.159 & 1.041 & 7.8 & 4753 & 987 & $7 \% \mathrm{Si}$ & Large $\mathrm{CuBe}$ \\
\hline TBFA05-03 & 0.147 & 1.041 & 8.9 & 3643 & 993 & $7 \% \mathrm{Si}$ & Large $\mathrm{CuBe}$ \\
\hline TBFA05-04 & 0.180 & 1.041 & 8.8 & 4515 & 995 & $7 \% \mathrm{Si}$ & Large $\mathrm{CuBe}$ \\
\hline TBFA05-05 & 0.145 & 1.041 & 9.0 & 3886 & 551 & $7 \% \mathrm{Si}$ & Large $\mathrm{CuBe}$ \\
\hline TBFA05-06 & 0.128 & 1.041 & 8.8 & 4143 & 556 & $7 \% \mathrm{Si}$ & Large $\mathrm{CuBe}$ \\
\hline TBFA05-07 & 0.110 & 1.041 & 8.7 & 3148 & 551 & $7 \% \mathrm{Si}$ & Large $\mathrm{CuBe}$ \\
\hline TBFA05-08 & 0.151 & 1.041 & 8.4 & 3004 & 544 & $7 \% \mathrm{Si}$ & Large $\mathrm{CuBe}$ \\
\hline TBFA05-09 & 0.123 & 1.041 & 8.8 & 3146 & 562 & $7 \% \mathrm{Si}$ & Large $\mathrm{CuBe}$ \\
\hline TBFA05-10 ${ }^{\dagger}$ & - & 0.610 & 4.7 & - & 887 & $7 \% \mathrm{Si}$ & Small CuBe \\
\hline TBFA05-11 ${ }^{\dagger}$ & 0.221 & 0.610 & 5.4 & 4947 & 915 & $7 \% \mathrm{Si}$ & Small CuBe \\
\hline TBFA05-12 ${ }^{\dagger}$ & 0.163 & 0.610 & 6.7 & 4951 & 885 & $7 \% \mathrm{Si}$ & Small CuBe \\
\hline TBFA05-13 & - & 0.787 & - & - & 408 & $7 \% \mathrm{Si}$ & Small CuBe \\
\hline TBFA05-14 ${ }^{\dagger}$ & 0.184 & 0.787 & 7.6 & 4840 & 894 & $7 \% \mathrm{Si}$ & Small CuBe \\
\hline TBSU05-01 & 0.207 & 0.787 & 7.1 & 3983 & 769 & $7 \% \mathrm{Si}$ & Large $\mathrm{CuBe}$ \\
\hline TBSU05-02 & 0.128 & 1.346 & 12 & 2128 & - & $7 \% \mathrm{Si}$ & Large $\mathrm{CuBe}$ \\
\hline TBSU05-03 & 0.089 & 0.787 & 8.8 & 3044 & 552 & $7 \% \mathrm{Si}$ & Large $\mathrm{CuBe}$ \\
\hline TBSU05-04 & 0.112 & 1.092 & 8.8 & 1737 & 475 & $7 \% \mathrm{Si}$ & Large $\mathrm{CuBe}$ \\
\hline TBSU05-05 & 0.096 & 1.092 & 8.9 & 1769 & 473 & $7 \% \mathrm{Si}$ & Large $\mathrm{CuBe}$ \\
\hline TBSU05-06 & 0.149 & 0.787 & 8.8 & 4097 & 485 & $7 \% \mathrm{Si}$ & Large $\mathrm{CuBe}$ \\
\hline TBSU05-07 & - & 0.787 & - & - & 516 & $7 \% \mathrm{Si}$ & Large $\mathrm{CuBe}$ \\
\hline TBSU05-08 & 0.161 & 0.787 & 8.8 & 4125 & 428 & $7 \% \mathrm{Si}$ & Large $\mathrm{CuBe}$ \\
\hline
\end{tabular}


Table B.1: (Continued)

\begin{tabular}{|c|c|c|c|c|c|c|c|}
\hline Cast ID & $\begin{array}{c}\mathrm{T} \\
(\mathrm{mm})\end{array}$ & $\begin{array}{c}\mathrm{G} \\
(\mathrm{mm})\end{array}$ & $\begin{array}{c}\mathrm{U} \\
(\mathrm{m} / \mathrm{s})\end{array}$ & $\begin{array}{c}\mathrm{P} \\
(\mathrm{Pa})\end{array}$ & $\begin{array}{c}\text { Mass } \\
(\mathrm{g})\end{array}$ & Alloy & Substrate \\
\hline TBSU05-09 & 0.149 & 0.787 & 8.8 & 4020 & 480 & $7 \% \mathrm{Si}$ & Large $\mathrm{CuBe}$ \\
\hline TBSU05-10 & 0.181 & 1.092 & 8.9 & 4134 & 479 & $7 \% \mathrm{Si}$ & Large $\mathrm{CuBe}$ \\
\hline TBSU05-11 & 0.191 & 1.041 & 8.8 & 4000 & 483 & $7 \% \mathrm{Si}$ & Large $\mathrm{CuBe}$ \\
\hline TBSU05-12 & 0.230 & 1.092 & 8.8 & 4958 & 468 & $7 \% \mathrm{Si}$ & Large $\mathrm{CuBe}$ \\
\hline TBSU05-13 & 0.285 & 0.787 & 5.7 & 3901 & 400 & $7 \% \mathrm{Si}$ & Large CuBe \\
\hline TBSU05-14 & 0.252 & 0.787 & 5.7 & 4053 & 488 & $7 \% \mathrm{Si}$ & Large $\mathrm{CuBe}$ \\
\hline TBSU05-15 & - & 0.787 & 5.7 & - & 340 & $7 \% \mathrm{Si}$ & Large $\mathrm{CuBe}$ \\
\hline TBSU05-16 & - & 0.787 & 5.7 & - & 457 & $7 \% \mathrm{Si}$ & Large $\mathrm{CuBe}$ \\
\hline TBSU05-17 & - & 0.787 & 5.7 & - & 398 & $7 \% \mathrm{Si}$ & Large $\mathrm{CuBe}$ \\
\hline TBSU05-18 & 0.122 & 0.787 & 7.2 & 2664 & 424 & $7 \% \mathrm{Si}$ & Large $\mathrm{CuBe}$ \\
\hline TBSU05-19 & 0.216 & 1.092 & 8.8 & 5421 & 434 & $7 \% \mathrm{Si}$ & Large $\mathrm{CuBe}$ \\
\hline TBSU05-20 & 0.195 & 1.092 & 8.8 & 5021 & 462 & $7 \% \mathrm{Si}$ & Large $\mathrm{CuBe}$ \\
\hline TBSU05-21 & 0.197 & 1.092 & 8.9 & 5177 & 350 & $7 \% \mathrm{Si}$ & Large $\mathrm{CuBe}$ \\
\hline TBSU05-22 & 0.144 & 0.787 & 7.1 & 3359 & 566 & $7 \% \mathrm{Si}$ & Large $\mathrm{CuBe}$ \\
\hline TBSU05-23 & 0.073 & 0.533 & 9.5 & 2797 & 372 & $7 \% \mathrm{Si}$ & Large $\mathrm{CuBe}$ \\
\hline TBSU05-24 & - & 0.533 & 7.1 & - & 334 & $7 \% \mathrm{Si}$ & Large $\mathrm{CuBe}$ \\
\hline TBSU05-25 & .078 & 0.406 & 9.6 & 3543 & 476 & $7 \% \mathrm{Si}$ & Large $\mathrm{CuBe}$ \\
\hline TBSU05-26 & - & 0.787 & 7.9 & - & 468 & Pure Al & Large $\mathrm{CuBe}$ \\
\hline TBSU05-27 & - & 0.787 & 7.9 & - & 585 & 1100 & Large $\mathrm{CuBe}$ \\
\hline TBSU05-28 & - & 0.787 & 7.9 & - & 750 & 1100 & Large $\mathrm{CuBe}$ \\
\hline TBSU05-29 & 0.124 & 0.787 & 7.8 & 2933 & 601 & $7 \% \mathrm{Si}$ & Large $\mathrm{CuBe}$ \\
\hline TBSU05-30 & 0.131 & 0.787 & 7.9 & 2889 & 751 & $7 \% \mathrm{Si}$ & Large $\mathrm{CuBe}$ \\
\hline TBSU05-31 & 0.092 & 0.787 & 9.5 & 2138 & 421 & $7 \% \mathrm{Si}$ & Large $\mathrm{CuBe}$ \\
\hline TBSU05-32 & - & 1.092 & 8.9 & - & 460 & $7 \% \mathrm{Si}$ & Large $\mathrm{CuBe}$ \\
\hline TBSU05-33 & 0.093 & 1.092 & 9.6 & 1783 & 459 & $7 \% \mathrm{Si}$ & Large $\mathrm{CuBe}$ \\
\hline TBSU05-34 & - & 1.092 & 8.8 & - & 468 & $7 \% \mathrm{Si}$ & Large $\mathrm{CuBe}$ \\
\hline TBSU05-35 & .107 & 0.787 & 7.8 & 3060 & 761 & 1100 & Large $\mathrm{CuBe}$ \\
\hline TBSU05-36 & - & 1.092 & 8.8 & - & 713 & 1100 & Large $\mathrm{CuBe}$ \\
\hline TBSU05-37 & 0.122 & 1.092 & 7.9 & 2214 & 620 & 1100 & Large $\mathrm{CuBe}$ \\
\hline TBSU05-38 & 0.162 & 1.092 & 8.9 & 3705 & 558 & $7 \% \mathrm{Si}$ & Large $\mathrm{CuBe}$ \\
\hline TBSU05-39 & 0.091 & 1.092 & 8.8 & 1893 & 567 & $7 \% \mathrm{Si}$ & Large $\mathrm{CuBe}$ \\
\hline TBSU05-40 & 0.130 & 0.787 & 12 & 4249 & 709 & 1100 & Large $\mathrm{CuBe}$ \\
\hline TBSU05-41 & 0.122 & 0.787 & 12 & 4247 & 707 & Pure Al & Large $\mathrm{CuBe}$ \\
\hline TBSU05-42 & 0.106 & 0.787 & 11.9 & 4044 & 708 & Pure Al & Large $\mathrm{CuBe}$ \\
\hline TBSU05-43 & 0.135 & 0.787 & 9.5 & 3958 & 707 & 1100 & Large $\mathrm{CuBe}$ \\
\hline TBSU05-44 & 0.097 & 0.787 & 8.8 & 3436 & 622 & $7 \% \mathrm{Si}$ & Small CuBe \\
\hline TBSU05-45 & 0.165 & 0.787 & 8.8 & 5063 & 711 & 1100 & Small CuBe \\
\hline TBSU05-46 & 0.098 & 0.787 & 11.9 & 2880 & 753 & 1100 & Small CuBe \\
\hline TBSU05-47 & 0.154 & 1.092 & 7.9 & 3982 & 750 & 1100 & Small CuBe \\
\hline TBSU05-48 & - & 1.092 & 5.6 & - & 712 & 1100 & Small CuBe \\
\hline TBSU05-49 & 0.109 & 0.787 & 7.2 & 3439 & 702 & 1100 & Small CuBe \\
\hline
\end{tabular}


Table B.1: (Continued)

\begin{tabular}{|c|c|c|c|c|c|c|c|}
\hline Cast ID & $\begin{array}{c}\mathrm{T} \\
(\mathrm{mm})\end{array}$ & $\begin{array}{c}\mathrm{G} \\
(\mathrm{mm})\end{array}$ & $\begin{array}{c}\mathrm{U} \\
(\mathrm{m} / \mathrm{s})\end{array}$ & $\begin{array}{c}\mathrm{P} \\
(\mathrm{Pa}) \\
\end{array}$ & $\begin{array}{c}\text { Mass } \\
(\mathrm{g})\end{array}$ & Alloy & Substrate \\
\hline TBSU05-50 & - & 0.787 & 6.4 & - & 707 & 1100 & Small $\mathrm{CuBe}$ \\
\hline TBSU05-51 & - & 0.787 & 9.5 & - & 713 & $7 \% \mathrm{Si}$ & Small CuBe \\
\hline TBSU05-52 ${ }^{\dagger}$ & 0.094 & 0.787 & 9.6 & 3941 & 696 & $7 \% \mathrm{Si}$ & Small CuBe \\
\hline TBSU05-54 ${ }^{\dagger}$ & 0.193 & 0.787 & 6.4 & 4113 & 689 & $7 \% \mathrm{Si}$ & Small CuBe \\
\hline TBSU05-55 ${ }^{\dagger}$ & 0.148 & 0.787 & 8.0 & 4323 & 714 & $7 \% \mathrm{Si}$ & Small $\mathrm{CuBe}$ \\
\hline TBSU05-56 ${ }^{\dagger}$ & 0.058 & 0.406 & 9.6 & 3877 & 551 & $7 \% \mathrm{Si}$ & Small CuBe \\
\hline TBSU05-57 ${ }^{\dagger}$ & - & 0.406 & 9.6 & - & 563 & $7 \% \mathrm{Si}$ & Small CuBe \\
\hline TBSU05-58 ${ }^{\dagger}$ & 0.082 & 0.406 & 9.6 & 5303 & 553 & $7 \% \mathrm{Si}$ & Small CuBe \\
\hline TBSU05-59 ${ }^{\dagger}$ & 0.072 & 0.406 & 9.6 & 4304 & 541 & $7 \% \mathrm{Si}$ & Small CuBe \\
\hline TBSU05-60 ${ }^{\dagger}$ & 0.141 & 1.118 & 8 & 3764 & 735 & $7 \% \mathrm{Si}$ & Small CuBe \\
\hline TBSU05-61 ${ }^{\dagger}$ & 0.183 & 1.118 & 7.2 & 3789 & 719 & $7 \% \mathrm{Si}$ & Small CuBe \\
\hline TBSU05-62 ${ }^{\dagger}$ & 0.206 & 1.321 & 7.2 & 2991 & 628 & $7 \% \mathrm{Si}$ & Small CuBe \\
\hline TBSU05-63 ${ }^{\dagger}$ & 0.079 & 0.533 & 88 & 4700 & 543 & $7 \% \mathrm{Si}$ & Small CuBe \\
\hline TBSU05-64 ${ }^{\dagger}$ & 0.121 & 0.406 & 9.5 & 7635 & 580 & $7 \% \mathrm{Si}$ & $1 \mathrm{CuBe}$ \\
\hline TBSU05-65 ${ }^{\dagger}$ & 0.153 & 0.610 & 9.6 & 6132 & 552 & $7 \% \mathrm{Si}$ & Small CuBe \\
\hline TBSP05-01 & - & 1.041 & - & - & 411 & $\begin{array}{c}7 \% \mathrm{Si} \\
4.7 \% \mathrm{Mg}\end{array}$ & $\mathrm{CuBe}$ \\
\hline TBSP05-02 & 0.180 & 1.041 & 7.2 & 3035 & 412 & $\begin{array}{c}7 \% \mathrm{Si} \\
4.7 \% \mathrm{Mg}\end{array}$ & Large $\mathrm{CuBe}$ \\
\hline TBSP05-03 & - & 1.016 & 7.2 & - & 509 & $\begin{array}{c}7 \% \mathrm{Si} \\
4.7 \% \mathrm{Mg}\end{array}$ & Large $\mathrm{CuBe}$ \\
\hline TBSP05-04 & 0.174 & 1.041 & 7.1 & 2989 & 473 & $\begin{array}{c}7 \% \mathrm{Si} \\
4.7 \% \mathrm{Mg}\end{array}$ & Large $\mathrm{CuBe}$ \\
\hline TBSP05-05 & 0.122 & 1.321 & 11.9 & 3108 & 607 & $7 \% \mathrm{Si}$ & Large $\mathrm{CuBe}$ \\
\hline TBSP05-06 * & - & - & - & - & 389 & $7 \% \mathrm{Si}$ & Large $\mathrm{CuBe}$ \\
\hline TBSP05-07 * & - & - & - & - & 353 & $7 \% \mathrm{Si}$ & Large $\mathrm{CuBe}$ \\
\hline TBSP05-08 * & - & - & - & - & 439 & $\begin{array}{l}7 \% \mathrm{Si} \\
7 \% \mathrm{Bi}\end{array}$ & Large $\mathrm{CuBe}$ \\
\hline TBS & - & - & - & - & - & $\begin{array}{l}7 \% \mathrm{Si} \\
7 \% \mathrm{Bi}\end{array}$ & $\mathrm{CuBe}$ \\
\hline TBSP05-10 & 0.126 & 0.787 & 8.7 & 1759 & 560 & $\begin{array}{l}7 \% \mathrm{Si} \\
4 \% \mathrm{Bi}\end{array}$ & Large $\mathrm{CuBe}$ \\
\hline TBSP05-11 & 0.113 & 1.092 & 8.4 & 1789 & 580 & $\begin{array}{l}7 \% \mathrm{Si} \\
4 \% \mathrm{Bi}\end{array}$ & Large $\mathrm{CuBe}$ \\
\hline TBSP05-12 & 0.097 & 1.346 & 11.7 & 2807 & 562 & $7 \% \mathrm{Si}$ & Large $\mathrm{CuBe}$ \\
\hline TBSP05-13 & 0.161 & 0.787 & 8.6 & 3561 & 690 & $7 \% \mathrm{Si}$ & Large $\mathrm{CuBe}$ \\
\hline TBSP05-14 & - & 1.041 & - & - & 483 & $7 \% \mathrm{Si}$ & Large $\mathrm{CuBe}$ \\
\hline TBSP05-15 & 0.213 & 1.041 & 6.1 & 2978 & 467 & $7 \% \mathrm{Si}$ & Large $\mathrm{CuBe}$ \\
\hline TBSP05-16 & 0.111 & 0.787 & 6.9 & 3581 & 518 & $7 \% \mathrm{Si}$ & Large $\mathrm{CuBe}$ \\
\hline TBSP05-17 & 0.099 & 0.787 & 8.7 & 1718 & 493 & $7 \% \mathrm{Si}$ & Large $\mathrm{CuBe}$ \\
\hline TBSP05-18 & 0.070 & 0.787 & - & - & 520 & $7 \% \mathrm{Si}$ & Large $\mathrm{CuBe}$ \\
\hline
\end{tabular}


Table B.1: (Continued)

\begin{tabular}{|c|c|c|c|c|c|c|c|}
\hline Cast ID & $\begin{array}{c}\mathrm{T} \\
(\mathrm{mm})\end{array}$ & $\begin{array}{c}\mathrm{G} \\
(\mathrm{mm})\end{array}$ & $\begin{array}{c}\mathrm{U} \\
(\mathrm{m} / \mathrm{s})\end{array}$ & $\begin{array}{c}\mathrm{P} \\
(\mathrm{Pa})\end{array}$ & $\begin{array}{c}\text { Mass } \\
(\mathrm{g})\end{array}$ & Alloy & Substrate \\
\hline TBSP05-19 & 0.078 & 0.787 & 8.6 & 1997 & 528 & $7 \% \mathrm{Si}$ & Large $\mathrm{CuBe}$ \\
\hline TBSP05-20 * & - & 1.346 & 11.8 & - & 488 & $7 \% \mathrm{Si}$ & Large $\mathrm{CuBe}$ \\
\hline TBSP05-21 * & - & 1.346 & 12.0 & - & - & $7 \% \mathrm{Si}$ & Large $\mathrm{CuBe}$ \\
\hline TBSP05-22 * & - & 1.346 & 11.9 & - & 601 & $7 \% \mathrm{Si}$ & Large $\mathrm{CuBe}$ \\
\hline TBSP05-23 * & 0.117 & 1.346 & 12 & 2300 & - & $7 \% \mathrm{Si}$ & Large $\mathrm{CuBe}$ \\
\hline TBSP05-24 & 0.070 & 0.787 & 8.6 & 1829 & 533 & $7 \% \mathrm{Si}$ & Large $\mathrm{CuBe}$ \\
\hline TBFA04-01 & 0.254 & 0.787 & 4.5 & 4456 & 462 & $\begin{array}{l}12.5 \% \mathrm{Si} \\
3 \% \mathrm{Mg}\end{array}$ & Large $\mathrm{CuBe}$ \\
\hline TBFA04-02 & 0.312 & 0.787 & 3.6 & 4403 & 527 & $\begin{array}{l}12.5 \% \mathrm{Si} \\
3 \% \mathrm{Mg}\end{array}$ & Large $\mathrm{CuBe}$ \\
\hline TBFA04-03 & 0.353 & 0.787 & 3.4 & 1998 & 598 & $\begin{array}{l}12.5 \% \mathrm{Si} \\
3 \% \mathrm{Mg}\end{array}$ & Large $\mathrm{CuBe}$ \\
\hline TBFA04-04 & 0.199 & 0.787 & 3.5 & 2395 & & $\begin{array}{l}12.5 \% \mathrm{Si} \\
3 \% \mathrm{Mg}\end{array}$ & Large $\mathrm{CuBe}$ \\
\hline TBFA04-05 & - & 1.041 & 3.4 & - & 532 & $\begin{array}{c}12.5 \% \mathrm{Si} \\
3 \% \mathrm{Mg}\end{array}$ & Large $\mathrm{CuBe}$ \\
\hline TBFA04-06 & - & 1.041 & 9.5 & - & - & $7 \% \mathrm{Si}$ & Large $\mathrm{CuBe}$ \\
\hline TBFA04-07 & - & 0.787 & 8.8 & - & 653 & $7 \% \mathrm{Si}$ & Large $\mathrm{CuBe}$ \\
\hline TBFA04-08 & - & - & 8.9 & - & - & $7 \% \mathrm{Si}$ & Large $\mathrm{CuBe}$ \\
\hline TBFA04-09 & - & 0.787 & 8.8 & - & 473 & $7 \% \mathrm{Si}$ & Large $\mathrm{CuBe}$ \\
\hline TBFA04-10 & - & 0.787 & 8.8 & - & 533 & $7 \% \mathrm{Si}$ & $\mathrm{CuBe}$ \\
\hline TBFA04-11 & - & 1.321 & 7.1 & - & 481 & $7 \% \mathrm{Si}$ & Large $\mathrm{CuBe}$ \\
\hline TBFA04-12 & - & 1.321 & 6.9 & - & 571 & $7 \% \mathrm{Si}$ & Large $\mathrm{CuBe}$ \\
\hline TBFA04-13 & 0.179 & 1.321 & 7.1 & 2858 & 508 & $7 \% \mathrm{Si}$ & Large $\mathrm{CuBe}$ \\
\hline TBFA04-14 & 0.170 & 1.321 & 7 & 2712 & 474 & $7 \% \mathrm{Si}$ & Large $\mathrm{CuBe}$ \\
\hline TBFA04-15 & 0.174 & 1.321 & 7.2 & 2973 & 516 & $7 \% \mathrm{Si}$ & Large $\mathrm{CuBe}$ \\
\hline TBFA04-16 & - & 1.321 & 3.8 & - & 500 & $7 \% \mathrm{Si}$ & Large $\mathrm{CuBe}$ \\
\hline TBFA04-17 & - & 1.321 & 4.7 & - & 500 & $7 \% \mathrm{Si}$ & Large $\mathrm{CuBe}$ \\
\hline TBFA04-18 * & - & 1.016 & 2.2 & - & 550 & Pure Al & Large $\mathrm{CuBe}$ \\
\hline TBFA04-19 * & - & 1.016 & 7.1 & - & 550 & Pure Al & Large $\mathrm{CuBe}$ \\
\hline TBFA04-20 * & - & 1.016 & 9.7 & - & 525 & Pure Al & Large $\mathrm{CuBe}$ \\
\hline TBFA04-21 * & - & 1.346 & 7.1 & - & 500 & $7 \% \mathrm{Si}$ & Large $\mathrm{CuBe}$ \\
\hline TBSU04-02 * & - & 1.041 & 9.5 & - & 511 & $7 \% \mathrm{Si}$ & Large $\mathrm{CuBe}$ \\
\hline TBSU04-03 * & - & 0.787 & 9.6 & - & 300 & $7 \% \mathrm{Si}$ & Large $\mathrm{CuBe}$ \\
\hline TBSU04-04 * & 0.135 & 0.660 & 8.8 & 2907 & 400 & $7 \% \mathrm{Si}$ & Large $\mathrm{CuBe}$ \\
\hline TBSU04-05 * & - & 0.660 & 8.8 & - & 400 & $7 \% \mathrm{Si}$ & Large $\mathrm{CuBe}$ \\
\hline TBSU04-06 * & - & 0.660 & 6.9 & - & 400 & $7 \% \mathrm{Si}$ & Large $\mathrm{CuBe}$ \\
\hline TBSU04-07 * & - & 0.787 & 7 & - & 351 & $7 \% \mathrm{Si}$ & Large $\mathrm{CuBe}$ \\
\hline TBSU04-08 * & - & 0.660 & 8.8 & - & 400 & $7 \% \mathrm{Si}$ & Large $\mathrm{CuBe}$ \\
\hline TBSU04-09 * & - & 0.660 & 8.8 & - & 400 & $7 \% \mathrm{Si}$ & Large $\mathrm{CuBe}$ \\
\hline TBSU04-10 * & - & 0.660 & 8.8 & - & 400 & $7 \% \mathrm{Si}$ & Large $\mathrm{CuBe}$ \\
\hline
\end{tabular}


Table B.1: (Continued)

\begin{tabular}{|c|c|c|c|c|c|c|c|}
\hline Cast ID & $\begin{array}{c}\mathrm{T} \\
(\mathrm{mm})\end{array}$ & $\begin{array}{c}\mathrm{G} \\
(\mathrm{mm})\end{array}$ & $\begin{array}{c}\mathrm{U} \\
(\mathrm{m} / \mathrm{s})\end{array}$ & $\begin{array}{c}\mathrm{P} \\
(\mathrm{Pa}) \\
\end{array}$ & $\begin{array}{c}\text { Mass } \\
(\mathrm{g}) \\
\end{array}$ & Alloy & Substrate \\
\hline TBSU04-11 & 0.112 & 0.787 & 11.9 & 4068 & 516 & $7 \% \mathrm{Si}$ & Large $\mathrm{CuBe}$ \\
\hline TBSU04-13 & .235 & 0.787 & 6.0 & 4348 & 518 & $7 \% \mathrm{Si}$ & Large $\mathrm{CuBe}$ \\
\hline TBSU04-14 & 0.235 & 0.787 & 6.0 & 4483 & 519 & $7 \% \mathrm{Si}$ & Large $\mathrm{CuBe}$ \\
\hline TBSU04-15 & 0.196 & 0.787 & 6.0 & 4579 & 518 & $7 \% \mathrm{Si}$ & Large $\mathrm{CuBe}$ \\
\hline TBSU04-16 & .092 & 0.787 & 14 & 4758 & 518 & $7 \% \mathrm{Si}$ & Large $\mathrm{CuBe}$ \\
\hline TBSU04-17 * & - & 0.787 & 8.8 & - & 524 & $7 \% \mathrm{Si}$ & Large $\mathrm{CuBe}$ \\
\hline TBSU04-18 * & - & 0.787 & 8.8 & - & 512 & $7 \% \mathrm{Si}$ & Large $\mathrm{CuBe}$ \\
\hline TBSU04-19 & - & 0.787 & 8.8 & - & 343 & Pure Al & Large $\mathrm{CuBe}$ \\
\hline TBSU04-20 * & - & 0.787 & & - & 458 & $7 \% \mathrm{Si}$ & Large $\mathrm{CuBe}$ \\
\hline TBSU04-21 & 0.157 & 1.041 & 8.8 & 4039 & 304 & Pure Al & Large $\mathrm{CuBe}$ \\
\hline TBSU04-22 * & - & 0.787 & 8.7 & - & 722 & $7 \% \mathrm{Si}$ & Large $\mathrm{CuBe}$ \\
\hline TBSU04-23 * & - & 0.787 & & - & 766 & $7 \% \mathrm{Si}$ & Large $\mathrm{CuBe}$ \\
\hline TBSU04-24 & 0.131 & 0.787 & 11.9 & 4864 & 1004 & $7 \% \mathrm{Si}$ & Large $\mathrm{CuBe}$ \\
\hline TBSU04-25 & 157 & 0.787 & 8.8 & 4436 & 599 & $7 \% \mathrm{Si}$ & $\mathrm{CuBe}$ \\
\hline TBSU04-26 & 0.208 & 0.787 & 7.1 & 3941 & 510 & $7 \% \mathrm{Si}$ & Large $\mathrm{CuBe}$ \\
\hline TBSU04-27 * & - & 0.787 & 8.7 & - & 435 & & $\mathrm{uBe}$ \\
\hline TBSU04-28 & 0.067 & 1.016 & 11.9 & 2139 & 735 & $7 \% \mathrm{Si}$ & $\mathrm{CuBe}$ \\
\hline TBSU04-29 & 0.152 & 0.787 & 8.8 & 4359 & 516 & $7 \% \mathrm{Si}$ & Large $\mathrm{CuBe}$ \\
\hline TBSU04-30 ${ }^{\dagger}$ & 0.222 & 0.787 & 5.7 & 4410 & 547 & $7 \% \mathrm{Si}$ & Large $\mathrm{CuBe}$ \\
\hline TBSU04-31 † & 0.126 & 0.787 & 11.9 & 4311 & 581 & $7 \% \mathrm{Si}$ & $\mathrm{CuBe}$ \\
\hline TBSP04-01 * & 0.126 & 1.016 & 8.8 & 2720 & 997 & $7 \% \mathrm{Si}$ & $\mathrm{CuBe}$ \\
\hline TBSP04-02 * & - & 1.016 & - & - & 1025 & $7 \% \mathrm{Si}$ & $\mathrm{CuBe}$ \\
\hline $4-03$ * & 135 & 1.016 & 8.8 & 3156 & 600 & & $\mathrm{uBe}$ \\
\hline TBS & 0.128 & 1.016 & 8.8 & 3120 & 732 & $\begin{array}{l}7 \% \mathrm{Si} \\
+\mathrm{WC}\end{array}$ & $\mathrm{uBe}$ \\
\hline TBSP04-05 * & - & 1.016 & 8.9 & - & 606 & $7 \% \mathrm{Si}$ & $\mathrm{CuBe}$ \\
\hline TBSP04-06 * & .159 & 1.016 & 8.9 & 3168 & 537 & $7 \% \mathrm{Si}$ & Large $\mathrm{CuBe}$ \\
\hline TBSP04-07 * & - & 1.016 & 8.8 & - & 534 & $7 \% \mathrm{Si}$ & Large $\mathrm{CuBe}$ \\
\hline TBSP04-08 * & .129 & 1.016 & 8.8 & - & - & $7 \% \mathrm{Si}$ & Large $\mathrm{CuBe}$ \\
\hline TBS & 0.095 & 0.787 & 8.9 & 3340 & 1262 & $\begin{array}{l}7 \% \mathrm{Si} \\
+\mathrm{WC}\end{array}$ & $\mathrm{uBe}$ \\
\hline TBSP04-10 * & 0.161 & 1.016 & 8.8 & 3602 & 566 & $7 \% \mathrm{Si}$ & Large $\mathrm{CuBe}$ \\
\hline TBSP04-11 * & 0.130 & 0.787 & 8.7 & 4388 & 512 & $7 \% \mathrm{Si}$ & Large $\mathrm{CuBe}$ \\
\hline TBSP04-12 * & 0.160 & 0.787 & 8.7 & - & - & $7 \% \mathrm{Si}$ & Large $\mathrm{CuBe}$ \\
\hline TBSP04-13 & 0.108 & 0.787 & 8.8 & 4647 & 423 & $7 \% \mathrm{Si}$ & Large $\mathrm{CuBe}$ \\
\hline TBSP04-14 * & 0.107 & 0.787 & 8.8 & 4923 & 528 & $7 \% \mathrm{Si}$ & Large $\mathrm{CuBe}$ \\
\hline TBSP04-15 * & 0.143 & 0.787 & 8.8 & 4074 & 478 & $7 \% \mathrm{Si}$ & Large $\mathrm{CuBe}$ \\
\hline TBSP04-16 & - & 0.787 & 8.9 & - & 656 & $\begin{array}{l}7 \% \mathrm{Si} \\
+\mathrm{SiC}\end{array}$ & Large $\mathrm{CuBe}$ \\
\hline TBSP04-17 & - & 1.016 & - & - & - & Pure Al & Large $\mathrm{CuBe}$ \\
\hline TBSP04-18 * & 0.154 & 0.787 & 8.8 & 3103 & 538 & $7 \% \mathrm{Si}$ & Large $\mathrm{CuBe}$ \\
\hline
\end{tabular}


Table B.1: (Continued)

\begin{tabular}{|c|c|c|c|c|c|c|c|}
\hline Cast ID & $\begin{array}{c}\mathrm{T} \\
(\mathrm{mm})\end{array}$ & $\begin{array}{c}\mathrm{G} \\
(\mathrm{mm})\end{array}$ & $\begin{array}{c}\mathrm{U} \\
(\mathrm{m} / \mathrm{s})\end{array}$ & $\begin{array}{c}\mathrm{P} \\
(\mathrm{Pa})\end{array}$ & $\begin{array}{c}\text { Mass } \\
(\mathrm{g})\end{array}$ & Alloy & Substrate \\
\hline TBSP04-19 * & 0.166 & 0.787 & 8.7 & 3519 & 445 & $7 \% \mathrm{Si}$ & Large $\mathrm{CuBe}$ \\
\hline TBFA03-01 & 0.140 & 1.321 & 8.8 & 2497 & 966 & $7 \% \mathrm{Si}$ & Large $\mathrm{CuBe}$ \\
\hline TBFA03-02 & 0.150 & 1.321 & 8.8 & 2791 & 994 & $7 \% \mathrm{Si}$ & Large $\mathrm{CuBe}$ \\
\hline TBFA03-03 & - & 1.321 & 8.8 & $2-$ & 943 & $7 \% \mathrm{Si}$ & Large $\mathrm{CuBe}$ \\
\hline TBFA03-04 & 0.134 & 1.321 & 8.9 & 2615 & 978 & $7 \% \mathrm{Si}$ & Large $\mathrm{CuBe}$ \\
\hline TBFA03-05 & 0.148 & 1.321 & - & - & 958 & $7 \% \mathrm{Si}$ & Large $\mathrm{CuBe}$ \\
\hline TBFA03-06 & - & 1.321 & 8.8 & - & 1008 & $7 \% \mathrm{Si}$ & Large $\mathrm{CuBe}$ \\
\hline TBFA03-07 & 0.146 & 1.321 & 8.9 & 2543 & 1000 & $7 \% \mathrm{Si}$ & Large $\mathrm{CuBe}$ \\
\hline TBFA03-08 * & 0.172 & - & 8.9 & 2998 & 992 & $7 \% \mathrm{Si}$ & Large $\mathrm{CuBe}$ \\
\hline TBFA03-09 * & 0.155 & 1.041 & 8.8 & 3092 & 1008 & $7 \% \mathrm{Si}$ & Large $\mathrm{CuBe}$ \\
\hline TBFA03-10 & 0.156 & 0.787 & 8.9 & 4165 & 1029 & $7 \% \mathrm{Si}$ & Large $\mathrm{CuBe}$ \\
\hline TBFA03-11 & 0.254 & 1.041 & 5.7 & 3263 & 982 & $7 \% \mathrm{Si}$ & Large $\mathrm{CuBe}$ \\
\hline TBFA03-12 & 0.223 & 1.041 & 5.5 & 2986 & 1010 & Pure Al & Large $\mathrm{CuBe}$ \\
\hline TBFA03-13 & - & 1.041 & - & - & 1043 & Pure Al & Large $\mathrm{CuBe}$ \\
\hline TBFA03-14 & - & 1.041 & 5.6 & - & 1065 & Pure Al & Large $\mathrm{CuBe}$ \\
\hline TBFA03-15 & - & 1.041 & 5.5 & - & - & Pure Al & Large $\mathrm{CuBe}$ \\
\hline TBFA03-16 & 0.211 & 1.041 & 4.3 & 2091 & 527 & Pure Al & Large $\mathrm{CuBe}$ \\
\hline TBFA03-17 & - & 1.041 & 4.2 & - & 491 & Pure Al & Large $\mathrm{CuBe}$ \\
\hline TBFA03-18 & - & 1.346 & 3.8 & - & 510 & Pure Al & Large $\mathrm{CuBe}$ \\
\hline TBFA03-19 & - & 1.041 & - & - & 523 & Pure Al & Large $\mathrm{CuBe}$ \\
\hline TBFA03-20 & - & 1.041 & 3.9 & - & 630 & Pure Al & Large $\mathrm{CuBe}$ \\
\hline TBFA03-21 & - & 1.041 & 3.8 & - & 624 & Pure Al & Large $\mathrm{CuBe}$ \\
\hline TBFA03-22 & - & 1.041 & 2.9 & - & 634 & Pure Al & Large $\mathrm{CuBe}$ \\
\hline TBFA03-23 & - & 1.041 & 2.6 & - & 534 & Pure Al & Large $\mathrm{CuBe}$ \\
\hline TBFA03-24 & - & 1.041 & 2.7 & - & 570 & Pure Al & Large $\mathrm{CuBe}$ \\
\hline TBFA03-25 & - & 1.041 & 2.6 & - & 586 & Pure Al & Large $\mathrm{CuBe}$ \\
\hline TBFA03-26 & .185 & 1.041 & 8.6 & 4071 & 586 & Pure Al & Steel \\
\hline TBFA03-27 & - & 1.041 & 1.5 & - & 611 & Pure Al & Large $\mathrm{CuBe}$ \\
\hline TBFA03-28 & - & 1.041 & 2.5 & - & 609 & Pure Al & Large $\mathrm{CuBe}$ \\
\hline TBFA03-29 & - & 1.041 & - & - & 564 & Pure Al & Large $\mathrm{CuBe}$ \\
\hline TBFA03-30 & 0.186 & 1.041 & 8.8 & 4344 & 636 & $7 \% \mathrm{Si}$ & Large $\mathrm{CuBe}$ \\
\hline TBFA03-31 & - & 1.041 & 8.8 & - & 641 & B356 & Large $\mathrm{CuBe}$ \\
\hline TBFA03-32 * & - & 1.016 & - & - & 945 & $7 \% \mathrm{Si}$ & Large $\mathrm{CuBe}$ \\
\hline TBSU03-01 & 0.174 & 1.092 & 8.8 & 3642 & 1009 & $7 \% \mathrm{Si}$ & Large $\mathrm{CuBe}$ \\
\hline TBSU03-02 & 0.160 & 1.092 & 8.8 & 3755 & 1016 & $7 \% \mathrm{Si}$ & Large $\mathrm{CuBe}$ \\
\hline TBSU03-03 & 0.088 & 0.787 & 9.8 & 3418 & 1035 & $7 \% \mathrm{Si}$ & Large $\mathrm{CuBe}$ \\
\hline TBSU03-04 & 0.129 & 0.787 & 9.8 & 4400 & 1001 & $7 \% \mathrm{Si}$ & Large $\mathrm{CuBe}$ \\
\hline TBSU03-05 & - & 0.787 & 9.5 & - & 984 & $7 \% \mathrm{Si}$ & Large $\mathrm{CuBe}$ \\
\hline TBSU03-06 & 0.190 & 0.787 & 9.5 & 553 & 1022 & $7 \% \mathrm{Si}$ & Large $\mathrm{CuBe}$ \\
\hline TBSU03-07 & 0.142 & 0.787 & 9.5 & 4184 & 1009 & $7 \% \mathrm{Si}$ & Large $\mathrm{CuBe}$ \\
\hline TBSU03-08 & 0.128 & 0.787 & 8.7 & 4264 & 1042 & $7 \% \mathrm{Si}$ & Large $\mathrm{CuBe}$ \\
\hline
\end{tabular}

${ }^{\dagger}=$ Gap measure, ${ }^{\ddagger}=$ Puddle length, ${ }^{\star}=$ modified nozzle, ${ }^{\diamond}=$ heated wheel 
Table B.1: (Continued)

\begin{tabular}{|c|c|c|c|c|c|c|c|}
\hline Cast ID & $\begin{array}{c}\mathrm{T} \\
(\mathrm{mm})\end{array}$ & $\begin{array}{c}\mathrm{G} \\
(\mathrm{mm})\end{array}$ & $\begin{array}{c}\mathrm{U} \\
(\mathrm{m} / \mathrm{s})\end{array}$ & $\begin{array}{c}\mathrm{P} \\
(\mathrm{Pa})\end{array}$ & $\begin{array}{c}\text { Mass } \\
(\mathrm{g})\end{array}$ & Alloy & Substrate \\
\hline TBSU03-09 & 0.134 & 0.787 & 8.8 & 4136 & 992 & $7 \% \mathrm{Si}$ & Large $\mathrm{CuBe}$ \\
\hline TBSU03-10 & 0.129 & 0.787 & 8.8 & 4309 & 964 & $7 \% \mathrm{Si}$ & Large $\mathrm{CuBe}$ \\
\hline TBSU03-11 & 0.149 & 0.787 & 8.8 & 4398 & 977 & $7 \% \mathrm{Si}$ & Large $\mathrm{CuBe}$ \\
\hline TBSU03-12 & 0.145 & 0.787 & 8.8 & 3959 & 987 & $7 \% \mathrm{Si}$ & Large $\mathrm{CuBe}$ \\
\hline TBSU03-13 & 0.165 & 0.787 & 8.8 & 4600 & 996 & $7 \% \mathrm{Si}$ & Large $\mathrm{CuBe}$ \\
\hline TBSU03-14 & 0.178 & 1.092 & 8.8 & 3611 & 1010 & $7 \% \mathrm{Si}$ & Large $\mathrm{CuBe}$ \\
\hline TBSU03-15 & - & 1.092 & 8.8 & - & 993 & $7 \% \mathrm{Si}$ & Large $\mathrm{CuBe}$ \\
\hline TBSU03-16 & 0.158 & 1.092 & 8.8 & 3547 & 988 & $7 \% \mathrm{Si}$ & Large $\mathrm{CuBe}$ \\
\hline TBSU03-17 & 0.163 & 1.092 & 8.8 & 3457 & 988 & $7 \% \mathrm{Si}$ & Large $\mathrm{CuBe}$ \\
\hline TBSU03-18 & 0.156 & 1.092 & 8.8 & 3260 & 997 & $7 \% \mathrm{Si}$ & Large $\mathrm{CuBe}$ \\
\hline TBSU03-19 & 0.157 & 1.092 & 8.8 & 3206 & 971 & $7 \% \mathrm{Si}$ & Large $\mathrm{CuBe}$ \\
\hline TBSU03-20 & - & 0.787 & 8.7 & - & 984 & $7 \% \mathrm{Si}$ & Large $\mathrm{CuBe}$ \\
\hline TBSU03-21 & 0.128 & 1.092 & 8.8 & 3231 & 1002 & $7 \% \mathrm{Si}$ & Large $\mathrm{CuBe}$ \\
\hline TBSU03-22 & - & 1.092 & 8.8 & - & 1040 & $7 \% \mathrm{Si}$ & Large $\mathrm{CuBe}$ \\
\hline TBSU03-23 & 0.194 & 1.092 & 8.8 & 3543 & 989 & $7 \% \mathrm{Si}$ & Large $\mathrm{CuBe}$ \\
\hline TBSU03-24 & - & 0.787 & 8.7 & - & 993 & $7 \% \mathrm{Si}$ & Large $\mathrm{CuBe}$ \\
\hline TBSU03-25 ${ }^{\dagger}$ & 0.158 & 1.092 & 8.7 & 3283 & 1015 & $7 \% \mathrm{Si}$ & Large $\mathrm{CuBe}$ \\
\hline TBSU03-26 & - & 0.787 & 8.8 & - & 997 & $7 \% \mathrm{Si}$ & Large $\mathrm{CuBe}$ \\
\hline TBSU03-27 & - & 0.000 & 8.8 & - & 980 & $7 \% \mathrm{Si}$ & Large $\mathrm{CuBe}$ \\
\hline TBSU03-28 ${ }^{\dagger}$ & 0.179 & 1.092 & 8.8 & 3742 & 993 & $7 \% \mathrm{Si}$ & Large $\mathrm{CuBe}$ \\
\hline TBSU03-29 & 0.179 & 0.787 & 8.8 & 4204 & 993 & $7 \% \mathrm{Si}$ & Large $\mathrm{CuBe}$ \\
\hline TBSU03-30 & 0.130 & 0.787 & 8.7 & 3696 & 960 & $7 \% \mathrm{Si}$ & Large $\mathrm{CuBe}$ \\
\hline TBSU03-31 & - & 1.092 & 8.8 & - & 972 & $7 \% \mathrm{Si}$ & Large $\mathrm{CuBe}$ \\
\hline TBSU03-32 & - & 0.787 & 8.7 & - & 982 & $7 \% \mathrm{Si}$ & Large $\mathrm{CuBe}$ \\
\hline TBSU03-33 & 0.111 & 0.533 & 8.8 & 3969 & 964 & $7 \% \mathrm{Si}$ & Large $\mathrm{CuBe}$ \\
\hline TBSU03-34 & 0.097 & 0.533 & 8.8 & 4897 & 993 & $7 \% \mathrm{Si}$ & Large $\mathrm{CuBe}$ \\
\hline TBSU03-35 & 0.103 & 0.533 & 8.8 & 5597 & 977 & $7 \% \mathrm{Si}$ & Large $\mathrm{CuBe}$ \\
\hline TBSU03-36 & 0.104 & 0.533 & 8.8 & 4995 & 986 & $7 \% \mathrm{Si}$ & Large $\mathrm{CuBe}$ \\
\hline TBSU03-37 & 0.124 & 0.533 & 8.9 & 5423 & 962 & $7 \% \mathrm{Si}$ & Large $\mathrm{CuBe}$ \\
\hline TBSU03-38 & 0.104 & 0.533 & 8.8 & 5817 & 979 & $7 \% \mathrm{Si}$ & Large $\mathrm{CuBe}$ \\
\hline TBSU03-39 & 0.110 & 0.533 & 8.9 & 5474 & 972 & $7 \% \mathrm{Si}$ & Large $\mathrm{CuBe}$ \\
\hline TBSU03-40 & 0.129 & 1.092 & 9.1 & 3283 & 965 & $7 \% \mathrm{Si}$ & Large $\mathrm{CuBe}$ \\
\hline TBSU03-41 & - & 1.092 & 8.8 & - & 988 & $7 \% \mathrm{Si}$ & Large $\mathrm{CuBe}$ \\
\hline TBSP03-01 * & 0.112 & 0.787 & 9.6 & 3640 & 1000 & $7 \% \mathrm{Si}$ & Large $\mathrm{CuBe}$ \\
\hline TBSP03-02 & 0.100 & 1.016 & 8.7 & 3985 & 1013 & $7 \% \mathrm{Si}$ & Large $\mathrm{CuBe}$ \\
\hline TBSP03-03 & 0.092 & 0.787 & 8.7 & 3892 & 1036 & $7 \% \mathrm{Si}$ & Large $\mathrm{CuBe}$ \\
\hline TBSP03-04 & 0.111 & 1.016 & 8.9 & 3908 & 1002 & $7 \% \mathrm{Si}$ & Large CuBe \\
\hline TBSP03-05 & 0.122 & 0.787 & 8.9 & 4108 & 984 & $7 \% \mathrm{Si}$ & Large $\mathrm{CuBe}$ \\
\hline TBSP03-06 & 0.109 & 0.787 & 8.9 & 4083 & 1011 & $7 \% \mathrm{Si}$ & Large $\mathrm{CuBe}$ \\
\hline TBSP03-07 & 0.121 & 0.787 & 8.8 & 3995 & 1017 & $7 \% \mathrm{Si}$ & Large $\mathrm{CuBe}$ \\
\hline TBSP03-08 & 0.121 & 0.787 & 8.9 & 4184 & 1001 & $7 \% \mathrm{Si}$ & Large $\mathrm{CuBe}$ \\
\hline
\end{tabular}

${ }^{\dagger}=$ Gap measure, ${ }^{\ddagger}=$ Puddle length, ${ }^{\star}=$ modified nozzle, ${ }^{\diamond}=$ heated wheel 
Table B.1: (Continued)

\begin{tabular}{|c|c|c|c|c|c|c|c|}
\hline Cast ID & $\begin{array}{c}\mathrm{T} \\
(\mathrm{mm})\end{array}$ & $\begin{array}{c}\mathrm{G} \\
(\mathrm{mm})\end{array}$ & $\begin{array}{c}\mathrm{U} \\
(\mathrm{m} / \mathrm{s})\end{array}$ & $\begin{array}{c}\mathrm{P} \\
(\mathrm{Pa})\end{array}$ & $\begin{array}{c}\text { Mass } \\
(\mathrm{g})\end{array}$ & Alloy & Substrate \\
\hline TBSP03-09 * & 0.124 & 0.787 & 8.9 & 2915 & 1016 & $7 \% \mathrm{Si}$ & Large $\mathrm{CuBe}$ \\
\hline TBSP03-10 * & 0.157 & 0.787 & 8.6 & 3570 & 1030 & $7 \% \mathrm{Si}$ & Large $\mathrm{CuBe}$ \\
\hline TBSP03-11 & 0.134 & 0.787 & 8.7 & 3718 & 988 & $7 \% \mathrm{Si}$ & Large $\mathrm{CuBe}$ \\
\hline TBSP03-12 & 0.156 & 0.787 & 8.8 & 4441 & - & $7 \% \mathrm{Si}$ & Large $\mathrm{CuBe}$ \\
\hline TBSP03-13 & - & 0.787 & 8.9 & - & 1000 & $7 \% \mathrm{Si}$ & Large $\mathrm{CuBe}$ \\
\hline TBSP03-14 & 0.156 & 0.787 & 8.7 & 3492 & 1014 & $7 \% \mathrm{Si}$ & Large $\mathrm{CuBe}$ \\
\hline TBSP03-15 & 0.160 & 0.787 & 8.7 & 3501 & 1014 & $7 \% \mathrm{Si}$ & Large $\mathrm{CuBe}$ \\
\hline TBSP03-16 & - & 0.787 & 8.7 & - & 1004 & $7 \% \mathrm{Si}$ & Large $\mathrm{CuBe}$ \\
\hline TBSP03-17 & - & 0.787 & & - & 984 & $7 \% \mathrm{Si}$ & Large $\mathrm{CuBe}$ \\
\hline TBSP03-18 & - & 1.092 & 8.7 & - & 1008 & $7 \% \mathrm{Si}$ & Large $\mathrm{CuBe}$ \\
\hline TBSP03-19 & - & 1.092 & - & - & 1003 & $7 \% \mathrm{Si}$ & Large $\mathrm{CuBe}$ \\
\hline TBSP03-20 & - & 0.787 & 9.5 & - & 1000 & Pure Al & Large $\mathrm{CuBe}$ \\
\hline TBSP03-21 & - & 0.787 & 8.8 & - & 1000 & $7 \% \mathrm{Si}$ & Large $\mathrm{CuBe}$ \\
\hline TBSP03-22 & - & 0.787 & 8.8 & - & 1022 & $7 \% \mathrm{Si}$ & Large $\mathrm{CuBe}$ \\
\hline TBSP03-23 & - & 0.787 & 8.6 & - & 1048 & $7 \% \mathrm{Si}$ & Large $\mathrm{CuBe}$ \\
\hline TBSP03-24 & - & 0.787 & 8.8 & - & 963 & Pure Al & Large $\mathrm{CuBe}$ \\
\hline TBSP03-25 & - & 0.787 & 8.8 & - & 1040 & Pure Al & Large $\mathrm{CuBe}$ \\
\hline TBSP03-26 & 0.176 & 1.092 & 8.8 & 3804 & 1005 & $7 \% \mathrm{Si}$ & Large $\mathrm{CuBe}$ \\
\hline TBSP03-27 & 0.155 & 1.092 & 8.7 & 3637 & 1003 & $7 \% \mathrm{Si}$ & Large $\mathrm{CuBe}$ \\
\hline TBFA02-01 & - & 1.016 & - & - & 1022 & $7 \% \mathrm{Si}$ & Large $\mathrm{CuBe}$ \\
\hline TBFA02-02 & - & 0.787 & 1.7 & - & 1134 & $7 \% \mathrm{Si}$ & Large $\mathrm{CuBe}$ \\
\hline TBFA02-03 & 0.128 & 0.787 & 9.5 & 4276 & 1154 & $7 \% \mathrm{Si}$ & Large $\mathrm{CuBe}$ \\
\hline TBFA02-04 & - & 0.787 & - & - & 1010 & $7 \% \mathrm{Si}$ & Large $\mathrm{CuBe}$ \\
\hline TBFA02-05 * & - & 0.787 & 9.4 & - & 1010 & $7 \% \mathrm{Si}$ & Large $\mathrm{CuBe}$ \\
\hline TBFA02-06 & 0.154 & 0.787 & 9.5 & 4044 & 956 & $7 \% \mathrm{Si}$ & Large $\mathrm{CuBe}$ \\
\hline TBFA02-07 & 0.119 & 0.787 & 9.5 & 4370 & 1150 & $7 \% \mathrm{Si}$ & Large $\mathrm{CuBe}$ \\
\hline TBFA02-08 & - & 0.787 & 9.6 & - & 1003 & $7 \% \mathrm{Si}$ & Large $\mathrm{CuBe}$ \\
\hline TBFA02-09 * & 0.139 & 0.787 & 9.5 & - & 996 & $7 \% \mathrm{Si}$ & Large $\mathrm{CuBe}$ \\
\hline TBFA02-10 * & 0.109 & 0.787 & 9.7 & 3314 & 1001 & $7 \% \mathrm{Si}$ & Large $\mathrm{CuBe}$ \\
\hline TBFA02-11 * & 0.110 & 0.787 & 9.3 & 3505 & 1019 & $7 \% \mathrm{Si}$ & Large $\mathrm{CuBe}$ \\
\hline TBFA02-12 & - & 0.787 & 9.6 & - & 992 & $7 \% \mathrm{Si}$ & Large $\mathrm{CuBe}$ \\
\hline TBFA02-13 * & - & 0.787 & 9.6 & - & 984 & $7 \% \mathrm{Si}$ & Large $\mathrm{CuBe}$ \\
\hline TBFA02-14 * & 0.098 & 0.787 & 9.5 & 3654 & 1015 & $7 \% \mathrm{Si}$ & Large $\mathrm{CuBe}$ \\
\hline TBFA02-15 * & 0.113 & 0.787 & 9.5 & 3657 & 1009 & $7 \% \mathrm{Si}$ & Large $\mathrm{CuBe}$ \\
\hline TBSU02-01 & 0.087 & 0.787 & - & - & 1044 & $7 \% \mathrm{Si}$ & Large $\mathrm{CuBe}$ \\
\hline TBSU02-02 & 0.200 & 0.787 & 8.9 & 4387 & 1008 & $7 \% \mathrm{Si}$ & Large $\mathrm{CuBe}$ \\
\hline TBSU02-03 & 0.163 & 0.787 & 8.8 & 4383 & 1008 & $7 \% \mathrm{Si}$ & Large $\mathrm{CuBe}$ \\
\hline TBSU02-04 & 0.084 & 0.787 & 8.9 & 5500 & 1008 & $7 \% \mathrm{Si}$ & Large $\mathrm{CuBe}$ \\
\hline TBSU02-05 & 0.149 & 0.787 & 9.1 & 4823 & 1024 & $7 \% \mathrm{Si}$ & Large $\mathrm{CuBe}$ \\
\hline TBSU02-06 & - & 0.787 & 8.8 & - & 1020 & $7 \% \mathrm{Si}$ & Large $\mathrm{CuBe}$ \\
\hline TBSU02-07 & 0.168 & 0.787 & 9.0 & 4759 & 1015 & $7 \% \mathrm{Si}$ & Large $\mathrm{CuBe}$ \\
\hline
\end{tabular}

${ }^{\dagger}=$ Gap measure, ${ }^{\ddagger}=$ Puddle length, ${ }^{\star}=$ modified nozzle, ${ }^{\diamond}=$ heated wheel 
Table B.1: (Continued)

\begin{tabular}{|c|c|c|c|c|c|c|c|}
\hline Cast ID & $\begin{array}{c}\mathrm{T} \\
(\mathrm{mm})\end{array}$ & $\begin{array}{c}\mathrm{G} \\
(\mathrm{mm})\end{array}$ & $\begin{array}{c}\mathrm{U} \\
(\mathrm{m} / \mathrm{s})\end{array}$ & $\begin{array}{c}\mathrm{P} \\
(\mathrm{Pa})\end{array}$ & $\begin{array}{c}\text { Mass } \\
(\mathrm{g})\end{array}$ & Alloy & Substrate \\
\hline TBSU02-08 & 0.149 & 0.787 & 9.0 & 4407 & 1026 & $7 \% \mathrm{Si}$ & Large $\mathrm{CuBe}$ \\
\hline TBSU02-09 & - & 0.787 & 9.0 & - & - & $7 \% \mathrm{Si}$ & Large $\mathrm{CuBe}$ \\
\hline TBSU02-10 & 0.138 & 1.016 & 10.7 & 4362 & 1021 & $7 \% \mathrm{Si}$ & Large $\mathrm{CuBe}$ \\
\hline TBSU02-11 & - & 1.016 & 10.5 & - & - & $7 \% \mathrm{Si}$ & Large $\mathrm{CuBe}$ \\
\hline TBSU02-12 & 0.137 & 1.016 & 10.2 & 4532 & 1026 & $7 \% \mathrm{Si}$ & Large $\mathrm{CuBe}$ \\
\hline TBSU02-13 & 0.153 & 1.016 & 10.2 & 4400 & 1012 & $7 \% \mathrm{Si}$ & Large $\mathrm{CuBe}$ \\
\hline TBSU02-14 & 0.121 & 1.016 & 10.3 & 4394 & 1015 & $7 \% \mathrm{Si}$ & Large $\mathrm{CuBe}$ \\
\hline TBSU02-15 & - & 1.016 & 10.3 & - & 1025 & $7 \% \mathrm{Si}$ & Large $\mathrm{CuBe}$ \\
\hline TBSU02-16 & 0.202 & 1.016 & 7.9 & 4809 & 1020 & $7 \% \mathrm{Si}$ & Large $\mathrm{CuBe}$ \\
\hline TBSU02-17 & - & 0.787 & - & - & 1042 & Pure Al & Large $\mathrm{CuBe}$ \\
\hline TBSU02-18 & 0.107 & 0.686 & 9.5 & 4819 & 1021 & $7 \% \mathrm{Si}$ & Large $\mathrm{CuBe}$ \\
\hline TBSU02-19 & 0.172 & 1.016 & 10.6 & 3755 & 1015 & $7 \% \mathrm{Si}$ & Large $\mathrm{CuBe}$ \\
\hline TBSU02-20 & 0.129 & 1.041 & 10.5 & 2970 & 1024 & $7 \% \mathrm{Si}$ & Large $\mathrm{CuBe}$ \\
\hline TBSU02-21 & - & 1.016 & 10.4 & - & 1015 & $7 \% \mathrm{Si}$ & Large $\mathrm{CuBe}$ \\
\hline TBSU02-22 & 0.180 & 1.016 & 9.6 & 3826 & 1015 & $7 \% \mathrm{Si}$ & Large $\mathrm{CuBe}$ \\
\hline TBSU02-23 & - & 1.016 & 9.5 & - & 1026 & $7 \% \mathrm{Si}$ & Large $\mathrm{CuBe}$ \\
\hline TBSU02-24 * & 0.102 & 1.016 & 9.5 & 2815 & 1022 & & Large $\mathrm{CuBe}$ \\
\hline TBSU02-25 * & 0.152 & 1.016 & 8.9 & 3352 & 1023 & $7 \% \mathrm{Si}$ & Large $\mathrm{CuBe}$ \\
\hline ETSP02-01 * & 0.175 & 0.787 & 8.9 & 4362 & 1044 & $7 \% \mathrm{Si}$ & Large $\mathrm{CuBe}$ \\
\hline ETSP02-02 * & - & 0.787 & 8.9 & - & 969 & $7 \% \mathrm{Si}$ & Large $\mathrm{CuBe}$ \\
\hline ETSP02-03 * & - & 0.787 & 8.9 & - & 1009 & $7 \% \mathrm{Si}$ & Large $\mathrm{CuBe}$ \\
\hline ETSP02-04 & - & 0.787 & 8.9 & - & 1008 & $7 \% \mathrm{Si}$ & Large $\mathrm{CuBe}$ \\
\hline ETSP02-05 & - & 0.787 & 8.8 & - & 1016 & $7 \% \mathrm{Si}$ & Large $\mathrm{CuBe}$ \\
\hline ETFA01-01 & 0.131 & 0.787 & 8.7 & - & 1023 & $7 \% \mathrm{Si}$ & Large $\mathrm{CuBe}$ \\
\hline ETFA01-02 & 0.186 & 0.787 & 8.8 & - & 1019 & $7 \% \mathrm{Si}$ & Large $\mathrm{CuBe}$ \\
\hline ETFA01-03 & 0.178 & 0.787 & 8.8 & - & 1029 & $7 \% \mathrm{Si}$ & Large $\mathrm{CuBe}$ \\
\hline ETFA01-04 & 0.172 & 0.787 & 8.8 & - & 1019 & $7 \% \mathrm{Si}$ & Large $\mathrm{CuBe}$ \\
\hline ETFA01-05 & - & 0.787 & - & - & 1008 & $7 \% \mathrm{Si}$ & Large $\mathrm{CuBe}$ \\
\hline ETFA01-06 & 0.141 & 0.787 & 8.8 & 4350 & 1018 & $7 \% \mathrm{Si}$ & Large $\mathrm{CuBe}$ \\
\hline ETFA01-07 & 0.159 & 0.787 & 8.8 & 4700 & 1007 & $7 \% \mathrm{Si}$ & Large $\mathrm{CuBe}$ \\
\hline ETFA01-08 & 0.159 & 0.787 & 8.9 & 4630 & 1012 & $7 \% \mathrm{Si}$ & Large $\mathrm{CuBe}$ \\
\hline ETFA01-09 & 0.120 & 0.787 & 8.9 & 5120 & 1015 & $7 \% \mathrm{Si}$ & Large $\mathrm{CuBe}$ \\
\hline ETFA01-10 & - & 0.787 & 8.7 & - & 1015 & $7 \% \mathrm{Si}$ & Large $\mathrm{CuBe}$ \\
\hline ETFA01-11 & - & 0.787 & 8.8 & - & 999 & $7 \% \mathrm{Si}$ & Large $\mathrm{CuBe}$ \\
\hline
\end{tabular}

${ }^{\dagger}=$ Gap measure, ${ }^{\ddagger}=$ Puddle length, ${ }^{\star}=$ modified nozzle, ${ }^{\diamond}=$ heated wheel 


\section{BIBLIOGRAPHY}

Al-Huniti, N. \& Al-NimR, M. 2000 Behavior of thermal stresses in a rapidly heated thin plate. J. Ther. Stresses 23, 293-307.

A.M.Korol'Kov 1956 Otdelenie Teknicheskik Nauk 2, 35-42.

Anthony, T. \& Cline, H. 1978 On the uniformity of amorphous metal ribbon formed by a cylindrical jet impinging on a flat moving substrate. J. Appl. Phys. 49, 829-837.

Anthony, T. \& Cline, H. 1979 Dimensional variations in newtonian-quenched metal ribbons formed by melt spinning and melt extraction. J. Appl. Phys. 50 (1), 245-254.

Badri, A., Natarajan, T., Snyder, C., Powers, K., Mannion, F., Byrne, M. \& Cramb, A. 2005 A mold simulator for continuous casting of steel: Part ii. the formation of oscillation marks during the continuous casting of low carbon steel. Metall. Mat. Trans. B 36B (3), 373-383.

Basaran, O. \& DePaoli, D. 1994 Nonlinear oscillations of pendant drops. Phys. Fluids 6 (9), 2923-2943.

Belden, R. H. 1985 Commercializing a new product. Chem. Eng. Prog. May $1985,27-29$.

Benjamin, T. B. \& Scott, J. 1979 Gravity-capillary waves with edge constraints. J. Fluid Mech. 92, 241.

Bessemer, H. 1865 US Patent 49035. 
B.G. Thomas, D. L. \& Ho, B. 1997 Effect of transverse depressions and oscillation marks on heat transfer in the continuous casting mold. In Sensors and Modeling in Materials Processing: Techniques and Applications (ed. R. R. S. Viswanathan \& J. Malas), pp. 117-142. Warrendale, PA: The Minerals, Metals and Materials Society.

Bian, X., Perlin, M., Schultz, W. \& Agarwal, M. 2003 Axisymmetric slosh frequencies of a liquid mass in a circular cylinder. Phys. Fluids 15 (12), $3659-3664$

Billingham, J. 2002 Nonlinear sloshing in zero gravity. J. Fluid Mech. 464, $365-391$.

Bisch, C., Lasek, A. \& Rodot, H. 1982 Comportement hydrodynamique de volumes liquides spheriques semi-libres en apesanteur simulee. J. Mec. Theor. Appl. 1, 165-184.

Blake, T. D. 2006 The physics of moving wetting line. J. Colloid Interace Sci. 299 (1), 1-13.

Bussmann, M., Mostaghami, J., Kirk, D. \& Graydon, J. 2002 A numerical study of steady flow and temperature fields within a melt spinning puddle. Int. J. Heat and Mass Trans. 45, 3997-4010.

Byrne, C., Kueck, A., Baker, S. \& Steen, P. H. 2007 In-situ manipulation of cooling rates during planar-flow melt spinning processing. Submitted for publication .

Byrne, C., Weinstein, S. \& Steen, P. H. 2006 Capillary stabilty limits for liquid metal in melt spinning. Chem. Eng. Sci. 61, 8004-8009. 
Carlsaw, H. S. \& Jaeger, J. C. 1959 Conduction of Heat in Solids, 2nd edn. Oxford: Clarendon Press.

Carpenter, J. K. \& Steen, P. H. 1992 Planar-flow spin-casting of molten metals: process behaviour. J. Mat. Sci. 27, 215-225.

Carpenter, J. K. \& Steen, P. H. 1997 Heat transfer and solidification in planar-flow melt-spinning: high wheelspeeds. Intl. J. Heat Mass Transfer 40 (9), 1993-2007.

Chen, C. W. \& Hwang, W. S. 1995 A three-dimensional fluid flow model for puddle formation in the single-roll rapid solidification process. Appl. Math. Modelling 19, 704-712.

Chen, J.-J. \& Tseng, A. 1999 Modeling and optimization of nozzle design in planar flow melt spinning. MED, Manufacturing Science and Engineering (ASME) 10, 79-85.

Coyle, D., Macosko, C. \& Scriven, L. 1990 The fluid dynamics of reveerse roll coating. AIChE Journal 36 (2), 161-174.

Daniel, S., Chaudhury, M. K. \& De Gennes, P. G. 2005 Vibration-actuated drop motion on surfaces for batch microfluidic processes. Langmuir 21, 42404248.

Das, S. K., Bye, R. L., Barth, E. P., Spaepen, F., Ramanan, V. R. V. \& V, T. 1997 Processing of thick ductile metallic glass. Mat. Sci. Eng. A 226-228, $1-7$. 
Davies, H. A. 1985 Solidification mechanisms in amophous and crystalline ribbon casting. In Rapildly Quenched Metals (ed. S. Steeb \& H. Warlimont), pp. 101106. Elsevier.

DePaoli, D., Feng, J., Basaran, O. \& Scott, T. 1994 Hysteresis in forced oscillations of pendant drops. Phys. Fluids 7 (6), 1181-1183.

Dussan V, E. B. 1979 On the spreading of liquids on solid surfaces: static and dynamic contact lines. Ann. Rev. Fluid Mech. 11, 371-400.

Duwez, P., Willens, R. \& Klement, W. 1960 J. Appl. Phys. 31, 1136-1137.

Emmerich, K. 1985 Ribbon formation of roller cast al-cu. In Rapildly Quenched Metals (ed. S. Steeb \& H. Warlimont), pp. 71-74. Elsevier.

Fiedler, H., Mühlbach, H. \& Stephani, G. 1984 The effect of the main processing parameters on the geometry of amorphous metal ribbons during planar flow casting (pfc). J. Mat. Sci. 19, 3229-3235.

Gong, Z., Wilde, P. \& Matthys, E. 1991 Numerical modeling of the planar flow melt-spinning process and experimental investigation of its solidification puddle dynamics. Int. J. Rapid Solid. 6, 1-28.

Goumiri, L., Joud, J., P.Desre \& Hicter, J. 1979 Surface-tension of binaryliquid alloys presenting characteristic of immiscibility - al-pb, al-bi, al-sn and zn-bi. Surf. Sci. 83 (2), 471-486.

Haga, T. \& Suzuki, S. $2003 a$ Melt ejection twin roll caster for the strip casting of aluminum alloy. J. Mat. Proc. Tech. 137, 92-95. 
HagA, T. \& Suzuki, S. $2003 b$ Single roll method for foil casting of the aluminum alloy. J. Mat. Proc. Tech. 137, 86-91.

Hasegawa, R. 1983 Glassy Metals: Magnetic Chemical and Structural Properties. Boca Raton, FL: CRC Press.

Hasegawa, R. 2001 Design and synthesis of magnetic structures. Physica B 299, $199-204$.

Hasegawa, R. 2004 Applications of amorphous magnetic alloys. Mat. Sci. Eng. A 375, 90-97.

Heichal, Y. \& Chandra, S. 2005 Predicting thermal contact resistance between molten metal droplets and a solid surface. J. Heat Trans. 127, 1269-1275.

Heichal, Y., Chandra, S. \& Bordatchev, E. 2005 A fast-response thin film thermocouple to measure rapid surface temperature changes. Exp. Ther. Fluid Sci. 30, 153-159.

Hilpert, M. \& Miller, C. T. 1999 Experimental investigation on the resonance of a liquid column in a capillary tube. J. Coll. Int. Sci. 219, 62-68.

Hirsa, A. H., Lopez, C. A., Laytin, M. A., Vogel, M. J. \& Steen, P. H. 2005 Low-dissipation capillary switches at small scales. Appl. Phys. Lett. 86, 014106.

Hocking, L. 1987 Damping of capillary-gravity waves at a rigid boundary. J. Fluid Mech. 179, 253. 
HuAnG, S. C. 1981 The effect of melt delivery conditions on the casting of amorphous metal ribbons. In In Proc. 4th Int. Conf. on Rapidly Quenched Metals, Sendai, Japan:The Japan Institute of Metals, pp. 65-68.

HuAnG, S. C. \& Fiedler, H. C. 1981 Effects of wheel surface conditions on the casting of amorphous metal ribbons. Metall. Trans. A 12A, 1107-1112.

IBARAKI, T. 1996 Planar-flow melt-spinning: Experimental investigation on solidification, dynamics of the liquid puddle, and process operability. Master's thesis, Cornell University.

Ibaraki, T. \& Steen, P. H. 1995 Planar-flow spin casting: Puddle dynamics and process behavior. In Modelling of Casting, Welding, and Advanced Solidification Processes VII (ed. M. Cross \& J. Campbell), pp. 889-895. Warrendale PA: TMS.

IbARAki, T. \& Steen, P. H. 1996 Fluid and heat flow in planar-flow meltspinning. In Melt spinning, strip casting and slab casting (ed. E. Matthys \& W. Truckner), pp. 159-171. Warrendale PA: TMS.

Jones, H. 1982 Rapid solidifictaion of metals and alloys. In Metallic Glasses (ed. J.H.Gilman \& H.J.Leamy), pp. 1-83. London: Institution of Metallurgists.

Jones, H. 1984 Review: The status of rapid solidification of alloys in research and application. J. Mater. Sci. 19, 1043-76.

Jones, H. 2001 A perspective on the development of rapid solidification and nonequilibrium processing and its future. Mater. Sci. Eng. A 304-306, 11-19.

KAHn, M. 2000 Unsteady behavior of the planar flow spin-casting process. Master's thesis, Cornell University. 
Karaaslan, T., Yigiter, A. I., Keskin, M. \& Dagdemir, Y. 1998 Investigation of structure, metallographic and micro-hardness properties of rapidly solidified al-12wt\%si alloy by melt spinning. Physica Scripta. 58, 182-184.

Katgerman, L. \& Dom, F. 2004 Rapidly solidified aluminium alloys by meltspinning. Mater. Sci. Eng. A 375-377, 1212-1216.

Kavesh, S. 1978 Principles of fabrication. In Metallic Glasses (ed. J.H.Gilman \& H.J.Leamy), pp. 36-73. Metals Park: ASM.

KidAmbi, B. R. \& Shankar, P. 2004 The effects of the contact angle on sloshing in containers. Proc. R. Soc. Lond. A 460, 2251.

KistleR, S. 1993 Hydrodynamics of wetting. In Wettability (ed. J. Berg), pp. 311-429. New York: M. Dekker.

Kukura, J., Ford, K., Singh, A., Steen, P. H. \& Ibaraki, T. 1995 Measurement of heat transfer coefficient in planar flow casting. In Simulations of Materials Processing: Theory, Methods, and Applications (ed. S.F.Shen \& P.Dawson), pp. 1153-57. Rotterdam: Balkema.

LAmB, H. 1932 Hydrodynamics, 6th edn. Cambridge: Cambridge University Press.

Legresy, J., Brechet, Y. \& Menet, P. 1997 On the origins of short wave length thickness variations of strips in melt overflow casting. Scripta Materialia 36 (12), 1437-1442.

Li, G. \& Thomas, B. 1996 Transient thermal model of the continuous singlewheel thin-strip casting process. Met. Mater. Trans. B 27B, 509-525. 
Liebermann, H. 1984 Ribbon-substrate adhesion dynamics in chill block meltspinning processes. Metall. Trans. B 15B, 155-161.

Lopez, C. A., LeE, C. \& Hirsa, A. H. 2005 Electrochemically activated adaptive liquid lens. Appl. Phys. Lett. 87, 134102.

Lord Rayleigh 1879 On the capillary phenomena of jets. Proc. R. Soc. Lond. A 29, 71-97.

Loulou, T., Artyukhin, E. A. \& Bardon, J. P. 1999 Estimation of the thermal contact resistance during the first stages of metal solidification process: Ii - experimental setup and results. Int. J. Heat Mass Trans. 42, 2129-2142.

LOWRY, B. 1996 Pressure and stress meansurement via image analysis (p-sia) of axisymmetric drops and liquid bridges. J. Col. Int. Sci. 176, 284-297.

MARINGER, R. 1988 Solidification on a substrate. Mat. Sci. and Eng 98, 13-20.

Meco, H. \& Napolitano, R. E. 2005 Upper-bound velocity limit for free-jet melt spinning. Mat. Sci. Forums 459-479, 3371-3376.

Meng, Y. \& Thomas, B. G. 2003 Heat transfer and solidification model of continuous slab casting: Con1d. Metall. Mater. Trans. B 34B (5), 685-705.

Minorsky, N. 1962 Nonlinear Oscillations. Princeton, NJ: Van Nostrand.

Motojima, S., Ohtsuka, Y., Kawajiri, S., Takahashi, Y. \& Sugiyama, K. 1979 Quenching efficiency of some splat-cooling devices. J. Mat. Sci. 14, $498-500$. 
Muhlbach, H., Stephani, G., Sellger, R. \& Fiedler, H. 1987 Cooling rate and heat transfer coefficient during planar flow casting of microcrystalline steel ribbons. Int. J. Rapid Solidif. 3, 83-94.

Myshkis, A., Babskit, V., Kopachevskit, N., Slobozhanin, L. \& Tyuptsov, A. 1987 Low-Gravity Fluid Mechanics. Berlin: Springer-Verlag.

Napolitano, R. E. \& Meco, H. 2004 The role of melt pool behavior in free-jet melt spinning. Met. Mater. Trans. A 35 (5), 1539-1553.

NARAsimhan, M. C. 1979 US Patent 4142571.

NuCOR 2002 Nucor steel's twin-roll steel casting process CASTRIP ${ }^{\circledR}$ : www.castrip.com.

PArk, C., Kim, W. \& PARK, G. 2003 Thermal analysis of the roll in the strip casting process. Mech. Res. Comm. 30, 297-310.

Perepezko, J. H. \& Hebert, R. J. 2002 Amorphous aluminum alloys - synthesis and stability. J. Minerals Metals and Meterials Society 54, 34-44.

Pimputkar, S. M., Carbonara, R. S., Rayment, J. J., McCall, J. L. \& Clauer, A. H. 1985 Comparison of the single roller and double roller processes for casting low carbon steel. In Rapildly Quenched Metals (ed. S. Steeb \& H. Warlimont), pp. 95- 100. Elsevier.

Praisner, T., Chen, J.-J. \& Tseng, A. 1995 An experimental study of process behavior in planar flow melt spinning. Met. Mater. Trans. B 26B, 1199-1208.

Pryds, N. \& HATtel, J. 1997 Numerical modelling of rapid solidification. Modeling Simul. Mater. Sci. Eng. 5, 451-472. 
REED, B. L. 2001 Planar-flow spin casting: Momentum transport, vorticity transport, and texture formation. PhD thesis, Cornell University.

Robertson, J. M., Brouha, M., Stel, H. H. \& van der Borst, A. J. C. 1985 Surface quality and thickness effects of amorphous ribbons for recording head applications. In Rapildly Quenched Metals (ed. S. Steeb \& H. Warlimont), pp. 79-82. Elsevier.

Ruhl, R. 1967 Cooling rates in splat cooling. Mat. Sci. Eng. 1, 313-320.

Smith, M. T. \& Saletore, M. 1986 Simple, low-cost planar flow casting machine for rapid solidification processing. Rev. Sci. Instrum 57 (8), 1647-1653.

Steen, P. H. \& Karcher, C. 1997 Fluid mechanics of spin casting. Ann. Rev. Fluid Mechanics 29, 373-397.

Strani, M. \& Sabetta, F. 1984 Free vibrations of a drop in partial contact with a solid support. J. Fluid Mech. 141, 233-247.

Sung, J. K., Kim, M. C., PArk, C. G. \& Kim, Y. S. 1994 Theoretical expectation of strip thickness in planar flow casting process. Mat. Sci. Eng. A181/A182, 1237-1242.

Thomson, J. \& Hunt, G. 1986 Nonlinear Dynamics and Chaos: Geometrical Methods for Engineers and Scientists. New York: John Wiley and Sons.

Tkatch, V. I., Limanovskit, A. I., Denisenko, S. N. \& Rassolov, S. G. 2002 The effect of the melt-spinning processing parameters on the rate of coolong. Mat. Sci. Eng. A 323, 91-96. 
Trinh, E. \& Wang, T. G. 1982 Large-amplitude free and driven drop-shape oscillation: experimental observations. J. Fluid Mech 122, 315-338.

Trinh, E., Zwern, A. \& Wang, T. G. 1982 An experimental study of smallamplitude drop oscillations in immiscible liquid systems. J. Fluid Mech 115, $453-474$.

Vogel, M. J., Ehrhard, P. \& Steen, P. H. 2005 The electroosmotic droplet switch: Countering capillarity with electrokinetics. PNAS 102, 11974-11979.

VoGt, E. 1987 On the heat transfer mechanism in the melt spinning process. Int. J. Rapid Solidif. 3, 131-146.

Vogt, E. \& Frommeyer, G. 1985 The influence of process parameters on the coolong rate of the meltspinning process. In Rapildly Quenched Metals (ed. S. Steeb \& H. Warlimont), pp. 63-66. Elsevier.

WANG, G. \& Matthys, E. 1991 Modelling of rapid solidification by melt spinning: effect of heat transfer in the cooling substrtate. Mater.Sci Eng. A 136, $85-97$.

Wang, G. \& Matthys, E. 2002 Experimental determination of the interfacial heat transfer during cooling and solidification of molten metal droplets impacting on a metallic substrate: effect of roughness and superheat. Int. J. Heat and Mass Trans. 45, 4967-4981.

WANG, W. \& QIU, H. H. 2002 Interface thermal conductance in rapid solidification process. Int. J. Heat Mass Trans. 45, 2043-2053.

WebB, R. 1880 Some applications of a theorem in solid geometry. Mess. Math. IX, 177 . 
Weinstein, S. \& Ruschak, R. 2004 Coating flows. Ann. Rev. Fluid Mech. 36, $29-53$.

Wilde, P. \& Matthys, E. 1992 Experimental investigation of the planar flow casting process: development and free surface characteristics of the solidification puddle. Mat. Sci. Eng. A150, 237-247.

Wilkes, E. D. \& Basaran, O. A. 1997 Forced oscillations of pendant (sessile) drops. Phys. Fluids 9 (6), 1512-1528.

Wu, S. L., Chen, C. W., Hwang, W. S. \& Yang, C. C. 1992 Analysis for melt puddle in the planar flow casting process - a mathematical modellong study. Appl. Math. Modelling 16, 394-403.

Yukumoto, M., Shibuya, K., Kan, T. \& Ito, Y. 1985 Analysis of the solidification process in the roller quenching method. In Rapildly Quenched Metals (ed. S. Steeb \& H. Warlimont), pp. 91-94. Elsevier. 